\title{
I-11739-0645
}

DOE/OR/01-2304

FINAL-CONCURRED

\section{Environmental Baseline Survey Report for the Title Transfer of Land Parcel ED-4 at the East Tennessee Technology Park, Oak Ridge, Tennessee}

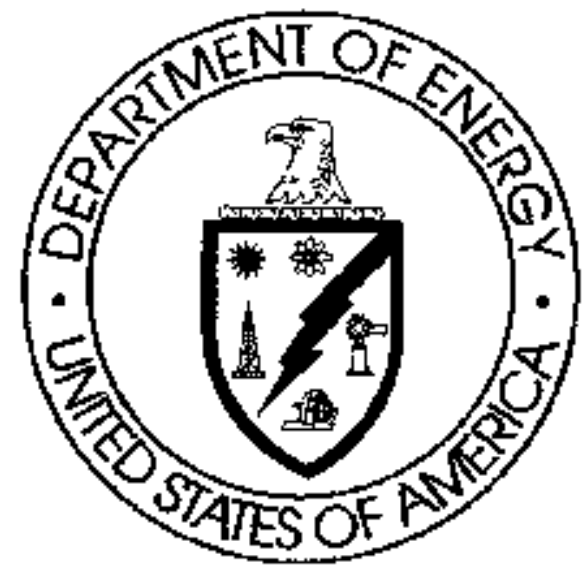

This document is apporoved for public telease per review by:

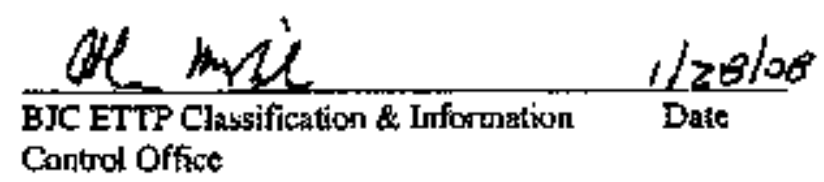




\section{SCIENCE APPLICATIONS INTERNATIONAL CORPORATION}

contributed to the preparation of this document and should not be considered an eligible contractor for its review. 


\section{EPA Concumence}




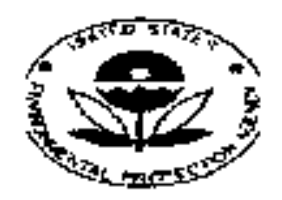

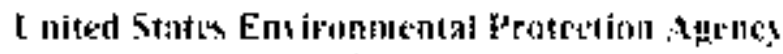

rish...

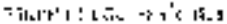

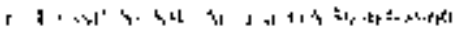

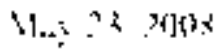

L.5

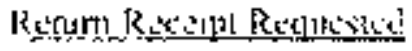

(4)ㄴ 3$)-1+P$

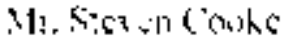

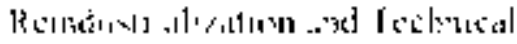

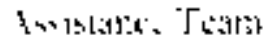

Depastumb at of tinerey

Oah Ridero Operalam bllit:

(') Bor zimil

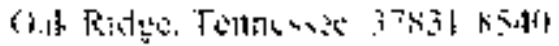

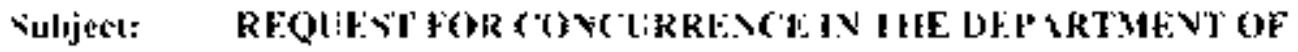

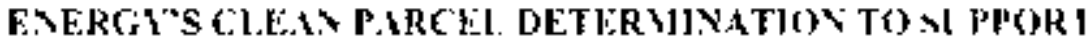

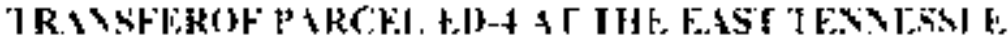
TECTNOLOF: I ARK. Vay 12, 20148

1):ar 11 . Couthe

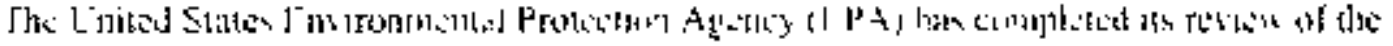

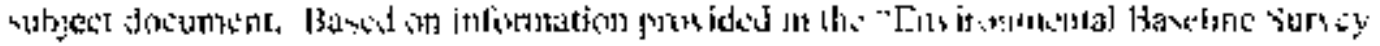

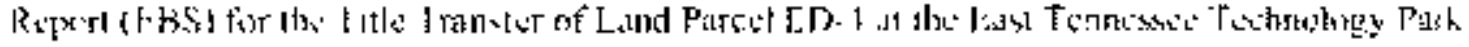

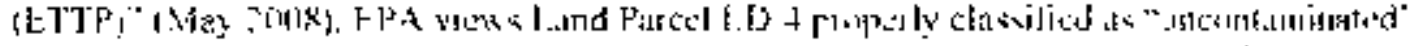

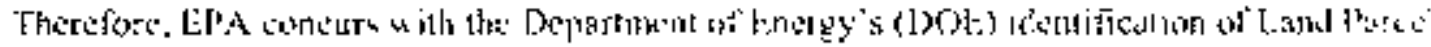

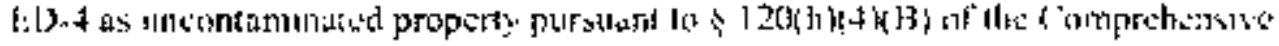

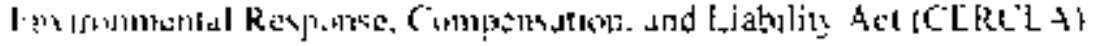


If you have any questions regarding this concurrence, please contact me at (404) 562-8543.

Sincerely,

Patricia J. Gokberg

Patricia J. Goldberg

Sr. Remedial Project Masager

Federal Facilities Branch

cc: Roger Petrie, TDEC

Dave Adler, DOE

Oak Ridge SSAB

Tim Mott, OSWER, OAA, FFRO

Jolm Reeder, OSWER, OAA, FFRO 
bc: Mantha Brock, OEA

Jeff Crane, FFB

Tim Frederick, TSS

Paye 5 of 3 


\section{Department of Energy}

Oak Ridge Operations Offlce

P.O. 80x 2001

Oak Ridge, Tennessee 37831-

May 12, 2008

Ms. Patricia Goldberg

U.S. Environmental Protection Agency

Region 4

Atlanta Federal Center

6l Forsyth Street

Attanta, Georgia $30303-8906$

Dear Ms. Gotdberg:

\section{REQUEST FOR CONCURRENCE DN THE DEPARTMENT OF ENERGY'S CLEAN PARCEL DETERMINATION TO SUPPORT TRANSFER OF PARCEL ED-4 AT THE EAST TENNESSEE TECHNOLOGY PARK}

The purpose of this letter is to request that Region 4 of the Environmental Protection Agency (EPA) concur in the Clean Parcel Determination that the Department of Energy (DOE) has made with regard to Parcel ED-4 at the East Tennessee Technology Park (ETTP). In addition, this letter transmits the revised Environnental Baselime Survey (EBS) Report that has been prepered to support this determination. Included in the EBS are responses to comments that were provided by EPA on March 19, 2008.

The EBS Report fncludes the results of an extensive records search as well as a physical inspection of the property, interviews with current and former workers, and exvironmental sempling. None of the investigative efforts identifies evidence that bazardous substances were released or disposed of on the subjest property, and the results of the risk evaluation indicate that occupation of the property is protective of buman health and the environment As a result, DOE has made the determination that Parcel ED-4 is suitable for release as uncontaminated property pussuant to Section 120(h)(4) of CERCLA. DOE requests that EPA concur with the Departuent's deternination that the property proposed for transfer is uncontaminated.

If you have any questions regarding DOE's Clean Parcel Determination or the enclosed information to support this determination, please contact Sue Carge, of ny staff, at (865) 576.0334.

Sincerely.

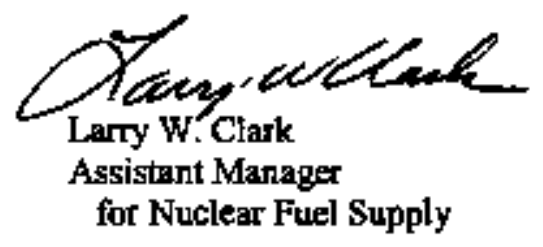

Enciosure

cc's on page 2 
c. wenclogure:

Sue Cange, NS-53, ORO

Steve Cooke, NS-53, ORO

Jennifer Tufts, EPA-Region 4

Tom Gebhart, TDECIDOE-0

Lawrence Young, CROET

ec wifo enclosire:

Steve McCracken, EM-90, ORO

Dennis Boggs, EM-90, ORO

David Adler, EM-91, ORO

Jack Howard, EM-93, ORO

Jun Kopotic, EM-93, ORO

Nancy Cames, CC-10, ORO

Cindy Finn, AD-42, ORO

Jeff Crane, EPA-Region 4

Roger Petrie, TDECJDOE-O

Lesley Cusick, BJC 
DOE/OR/01-2304

FINAL-CONCURRED

\title{
Environmental Baseline Survey Report for the Title Transfer of Land Parcel ED-4 at the East Tennessee Technology Park, Oak Ridge, Tennessee
}

Date Issuet-May 2008

\author{
Prepared by \\ Science Applications Intemational Corporation \\ Oak Ridge, Tennessee \\ under subcontract 23900-BA-PR007U \\ under work release 000900 \\ Prepared for the \\ U. S. Departaent of Energy \\ Office of Nuclear Euel Supply \\ BECHTEL JACOBS COMPANY LLC \\ managing the \\ Environmental Manifgement Activitits at the \\ East Temesset Technology Park \\ Y-12 National Security Complex Oak Ridge National Laboratory \\ under contract DE-ACOS-98OR22\%00 \\ for the \\ U. S. DEPARTMENT OF ENERGY
}


This report has been prepared by Science Applications International Corporation (SAJC) for the sole and exclusive use of Bechtel Jacobs Company LLC (BJC) and the U. S. Department of Energy. Any other person or entity obtaining, using, or relying on this report hereby acknowledges that they do so at their own risk, and that $S A C$ shall have no responsibility or liability for the consequences thereof. This report is prepared by $S A I C$ in accordance with the Comprehensive Environmental Response. Compensotion, and Liability Act of 1980 (CERCLA) $120(\mathrm{~h})(\mathrm{l})$ and (4) requirements.

This report is intended to be used in its entirety. Excerpts, which are taken out-of-context, run the risk of being misinterpreted and are therefore, not representative of the findings of this assessment. Opinions and recommendations presented in this report apply onty to site condittons and features as they existed at the time of SAYC's site visit, and those inferred from information observed or awilable at that sime, and camot be applied to conditions and features of which SAIC is unaware and has not had the opportunity to evaluate.

The results of this report are based on record reviews, site reconnaissance, interviews, and the radiological report reviewed and approved by BJC. $S A J C$ has not made, nor has it been asked to meske, any independent investigation concerning the acturacy, reliability, or completeness of stich information.

All sources of information on which SAIC has relied in making its conclusions are identified in Chap. 7 of this report. Any information, regardless of its source, not listed in Chap. 7 has not been evaluated or relied upon by SAIC in the context of this report. 


\section{CONTENTS}

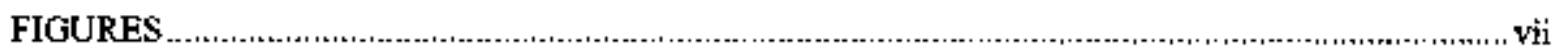

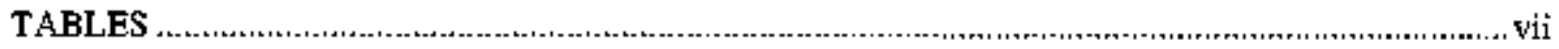

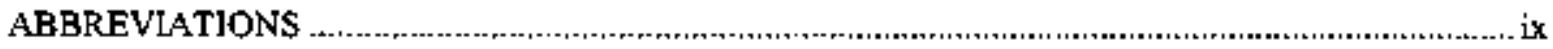

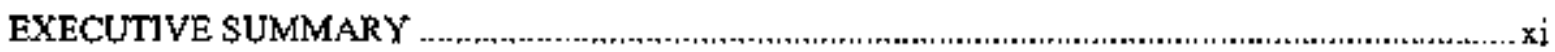

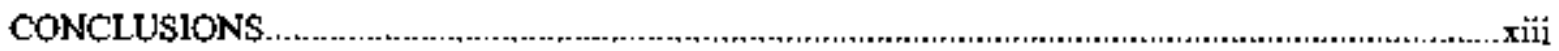

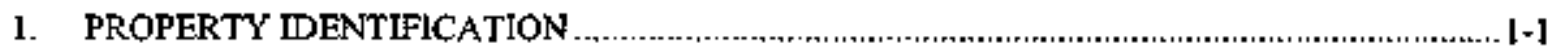

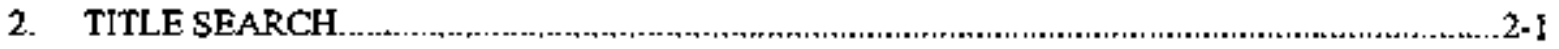

3. FEDERAL RECORDS SEARCH AND REGULATORY SUMMARY …..............................

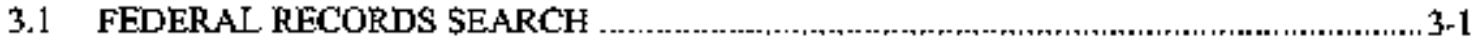

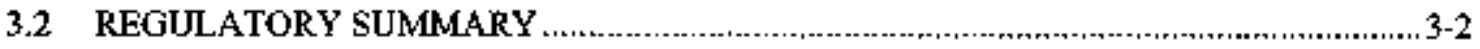

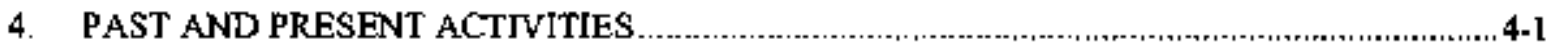

4.1 PAST AND PRESENT ACTIVITIES FOR THE REAL PROPERTY PROPOSED

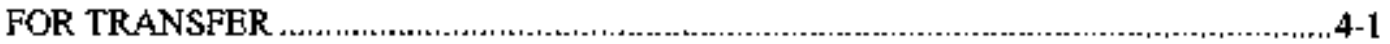

4.2 PAST AND PRESENT ACTIVITIES FOR THE ADJACENT PROPERTY ...................4-1

4.3 HYDROGEOLOGIC ENVTRONMENT …...........................................................

5. RESULTS OF VISUAL AND PHYSICAL INSPECTIONS ............................................5-1

5.1 VISUAL AND PHYSICAL INSPECTIONS OF THE PROPERTY FOR TRANSFER ........ $5-1$

5.2 VISUAL AND PHYSICAL INSPECTIONS OF ADJACENT PROPERTY ...................... 5-1

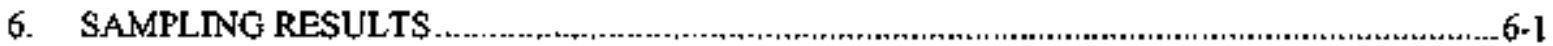

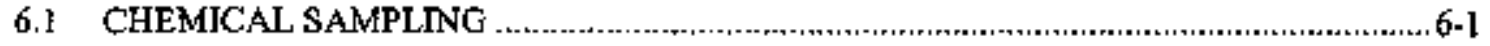

6.1.1 Data Validation..................................................................................................6-1

6.1.2 Chemical Sampling Results - Sediment .............................................................. 6-2

6.1.3 Chemical Sampling Results - Soil .................................................................6-10

6.1.4 Chemical Sampling ResuIts - Groundwater ........................................................6-16

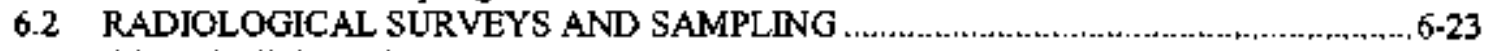

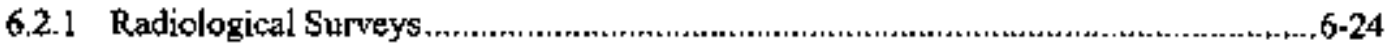

6.2 .2 Radiological Sampling …...........................................................................6-27

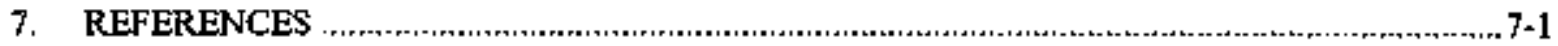

APPENDIX A REAL ESTATE ACQUSITION LETTER

APPENDIX B STUDY AREA MAP FROM RECORDS SEARCH................................................ B-1

APPENDIX C SAMPLING AND ANALYSIS PLAN FOR LAND PARCEL ED-4 AT THE

EAST TENNESSEE TECHNOLOGY PARK ..................................................................

APPENDIX D WALKOVER ASSESSMENT WORK INSTRUCTION ........................................ D-1 
APPENDIX E RADIOLOGICAL SURVEY PLAN FOR PARCEL ED-4 …................................. E-I

APPENDIX F RISK EVALUATION FOR PARCEL ED-4 ..................................................... 


\section{FIGURES}

1.1 Location map of the Parcel ED-4 study area........................................................................... 1-2

1.2 Circa 1945 atrial photo showing historical activities within a portion of the ED-4 footprint. ......... 1-3

4.1 Geologic and topographic trap of the ED-4 area. ................................................................ 4-3.

6.1 Sediment and surface soil sampling locations at ED-4 ......................................................

6.2 Groundwater sampling locations and results for VOCs at ED-4 .....................................

\section{TABLES}

6.1 Parcel ED-4 sediment chemical analytical results summary................................................6-2

6.2 Parcel ED-4 sediment - polychlorinated biphenyls results ......................................................64

6.3 Parcel ED-4 sedinent - volatile organic compounds results ..................................................6-5

6.4 Parcel ED-4 sediment - semivolatile organic compounds results .............................................6-6

6.5 Parcel ED-4 study area sediment - metals results ..................................................................

6.6 Parcel ED-4 soil chemical analytical results summary ..........................................................6-10

6.7 Parcel ED-4 soil - polychlorinated biphenyls results .............................................................6-11

6.8 Parcel ED-4 soil - volatile organic compounds results .........................................................6-12

6.9 Parcel ED-4 soil - semivolatile organic compoumds results ...............................................6-13

6.10 Parcel ED-4 study area soil - metals results ..................................................................6-15

6.11 Groundwater sample collection locations for Parcel ED-4 ......................................................6-17

6.12 Analytical results for VOCs in groundwater at Parcel ED-4 ..............................................6-19

6.13 Analytical results for metals in groundwater at Parcel ED-4 ...............................................6-20

6.14 Summary of analytical results for the Happy Valley Spring ..............................................6-22

6.15 Summery of analytical tesults for Spring 27-160 ......................................................... 6-23

6.16 Summary of contamination and dose rates ...................................................................6-25

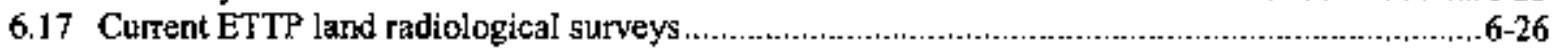

6.18 Fixed-timed radiological survey measurements of ED-4 …............................................6-27

6.19 Radiological soil sample data from Parcel ED-4 ...............................................................6-30

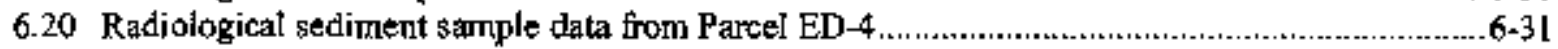

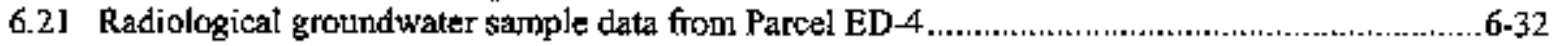

6.22 Parcel ED-4 summary statislics for radionuclides in soil .....................................................6.33

6.23 Parcel ED-4 summary statistics for radionuclides in sediment............................................ 6-34

6.24 Radionuclides summary for groundwater from Parcel ED-4 ................................................6-35

6.25 Radiological spring sample data from Happy Valley collected by the state of Tennessee...........6-37 


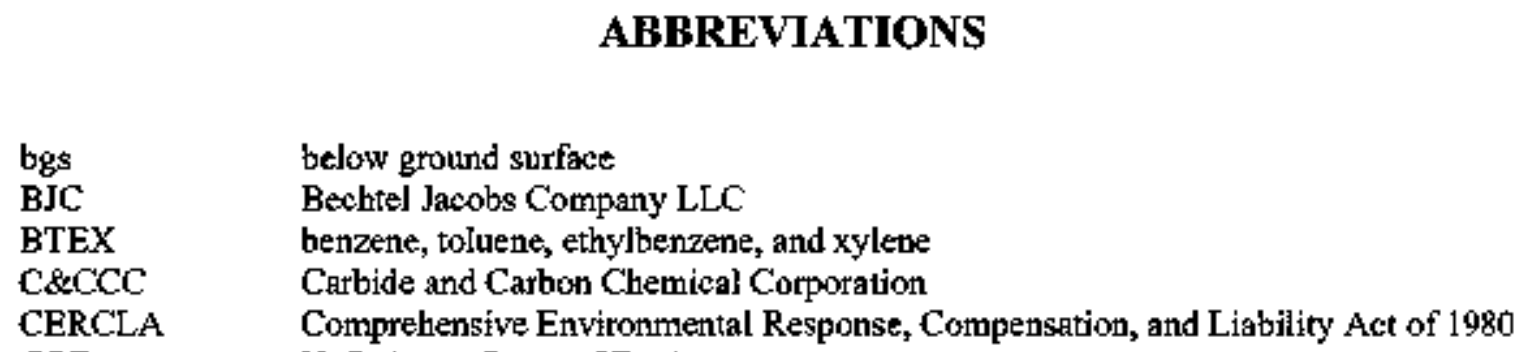




\section{EXECUTIVE SUMMARY}

This emvironmental baseline survey (EBS) report documents the baseline environmental conditions of a land parcel referred to as "ED 4" (ED-4) at the U.S. Department of Energy's (DOE's) East Tennessee Technology Park (ETTP). DOE is proposing to transfer the title of this land to the Heritage Center, LLC.

Parcel ED 4 is a land parcel that consists of two noncontiguous areas comprising a total of approxinately 18 acres located east of the ETTP. The western tract of ED-4 encompasses approximately 8.5 acres in the northeastem quadrant of the intersection of Boulevard Road and Highway 58 . The eastern tract encompasses an area of approximately 9.5 acres in the northwestern quadrant of the intersection of Blair Road and Highway 58 (the Oak Ridge Tumpike). Aerial photographs and site maps fiom throughout the bistory of the ETTP, going back to its initial development in the 1940s as the Oak Ridge Gaseous Diffusion Plant (ORGDP), indicate that this area has been undeveloped woodland with the exception of three support facilities for workers constructing the ORGDP since federal acquisition in 1943. These thres support facilitits, which were located in the western tract of ED 4, included a recreation ball, the Town Hall Camp Operations Building, and the Property Warehouse. A railroad spur also formerly occupied a portion of Parcel ED-4. These former facilities only occupied approximately 5 percent of the total area of Parcel ED-4.

This report provides supporting information for the transfer of this govenument-owned property at ETTP to a non-federal entity. This EBS is based upon the requirements of Sect 120(h) of the Comprehensive Environmental Response, Compensation, and Liability Acl of 1980 (CERCLA). In order to support a Clean Parcel Detemination (CPD) in accordance wilh CERCLA Seet 120(h)(4)(d), groundwater and sediment sarmples were collected within, and adjacent to, the Parcel ED-4 study arca The potential for DOE to make a CPD for ED-4 is further supported by a No Further Investigation (NFI) delermination made on land that adjoins ED-4 to the aast (DOE 1997a) and to the south (DOE 1997b).

Preparation of this report included the detailed search of Federal Govennent reconds [in accordance with CERCLA 120(h)(4)(A)(I)], title documents, aerial photos that may neflect prior uses, and physical and visual inspections of the property and adjacent propertics. Interviews with current employees' involved in operations on the real property were also conducted to idenlify any areas on the property where hazardous substances and petroleum products or their derivatives and acutely hazardous wastes were known to have been released, or disposed. In addition, a search was made of reasonably obtainable federal, state, and local government records of each adjacent facility where there has been a release of any hazardous substance or any petroleum product or their derivatives, including aviation fuel and motor oil, and which is likely to cause or contribute to a release of any hazardous substance or any petrolemn producl or its derivatives, including aviation fuel or motor oil on the real property proposed for transfer as a Clean Parcel (ED-4). Radiological surveys were conducted to assess the property's radiological condition, and soil sampling was also conducted to assess baseline conditions. The following is a summary of the findings of the evaluation that was performed:

- No cvidence was found of a release or disposal of hazardous substances or petroleum products or their derivatives oceurting on ED-4 property.

- There were and are no underground storage tanks located on ED4.

\footnotetext{
Park).

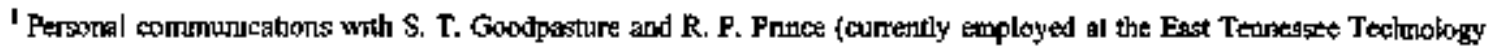


- No potential groumdwater plume bas been identified within, or in proxirnity to, ED-4. Additionally, the study area is upgradient of, or cross gradient to, the discharge zone for groundwater flow from ETTP.

- Parcel ED-4 is not located within ETTP Zones 1 or 2, which were established to address the potentially impacted areas of ETTP (DOE 2002); thus, no cleanup levels have been established for areas outside of Zones 1 or 2 , which includes the area oceupied by Parcel ED-4. However, for comparison purposes, Zone 1 rentediation levels (RLs) have been used for screening analytical results for soils and sediments at ED-4. In addition, residential risk-based preliminary remediation goals (PRGs) have also been used for screening the ED-4 soil and sediment data because the property is proposed for transfer as a Clean Parcel.

- Sediment and surface soil samples were collected in June of 2007 to support transfer of Parcel ED-4. The established Zone 1 RLs were not exceeded in either the sediment or soil samples collected at ED-4. Soil and sediment sampling results are discussed in Sect. 6.1 of this report.

- Results of the radiological surveys perforned in the study area and the statistical tests performed on the data gathered in each survey unit indicate that all results were less than the derived concentration guideline level (DCGL), and, therefore, the survey units can be transfened from a surface contamination standpoint. The radiological survey results are discussed in Sect. 6.2 of this report.

- The ED-4 transfer area soil/sediment data were screned against established RLs for ETTP Zone I (though ED-4 is not within either Zone 1 or Zone 2) and residential risk-based PRGs. No RLs were exceeded by soils'sediment at Parcel ED-4, and the PRG screen indicated that adverse health effects associaled with soilsediment residential exposure are highly unlikely. The analytical results for Parcel ED-4 soils and sediment indicated the cumulative risks were within the acceptable risk range and the $\mathrm{Hl}$ did not exceed $\mathrm{l}$ based on residential exposures. ln addition, because sample coliection was targeted to locations with the grealest potential for contamination (either at the location of historical operations, or from sediment accumblation areas within the parcel), it is unlikely that higher concentrations would be encountered within the parcel.

Because the risk evaluation for the residential scenario is within the J. S. Environmental Protection Agency's generally acceptable target risk range and the $\mathrm{HI}$ did not exceed 1 , and based on the information set forth in this document, DOE has identified the property proposed for transfer as "uncontaminated property" in accordance with CERCLA Set. 120(b)(4)(A). 


\section{CONCLUSIONS}

Based on the U.S. Department of Energy's (DOE's) review of the existing infomation, including discussions and interviews referenced berein, and evaluation of the data gathered in preparation of the environmental baseline survey for Parcel ED-4, DOE has determined that the parcel satisfies the statulory criteria for identification of the parcel as uncontaminated and that the transfer of Parcel ED-4 can be achieved by a Clean Parcel Detennination per Comprehensive Environmental Response, Compensation, and Liability Act of 1980 (CERCLA) Sect. 120(h)(4). Evidence that supports the conciusion includes the fact that no target organ hazard index exceedance occurred and the risk values are within the U. S. Environmental Protection Agency*s generally acceptable risk range (i.e., E-04 to E-06).

In addition, no evidence was found that hazardous substances were released, or disposed of, on the property that would preclude its identification as an uncentaminated parcel. Also, No Further Investigation (NFI) determinations have been made on property adjacent to the eastern and southern boundaries of Parcel ED-4. The property immediately adjacent to the nothem boundary of ED-4 was, and remains, undeveloped. The area bordering the eastem boumdary is occupied by a paved access road to the East Tennessee Technology Park and a parking lot. Thus, there are no indications of potential impacts on ED-4 from adjacent property that would preclude its identification as a Clean Parcel under CERCLA $120(b)(4)$.

\section{RESPONSES TO REGULATOR COMMENTS ON THE ENVIRONMENTAL BASELINE SURVEY REPORT FOR LAND PARCEL ED-4}

DOE received comments from the U.S. Environmental Protection Agency (EPA) on the Environmental Baseline Survey (EBS) report for Parcel ED-4 on March 19, 2008. Comments and responses are noted below.

1. Page 1-3. The ED-4 boundary reflected in the figure is not accurate. Please modify this figure.

DOE RESPONSE: The label for the boundary in the figure has been revised to note that it is the "ED-4 area". (It should also be noted that adjustrnents have been made to the proposed transfer footprints from DOE's initial notification of the intent to transfer that was issued in April 2007. The primary change to ED-4 was the reduction in the size of the footprint of the eastern parcel to avoid the wetlands. The notification letter was intended to commumicate DOE's overail plan, other minor changes may occur prior to transfer, especially to accommodate the metes and bounds survey.)

2. Page 4-2. The hydrogeology disclssed in Appendix $C$ should be included in Section 4.3. The hydrogeologic discussion in Appendix C, $5^{\text {th }}$ paragraph, discusses regional shallow groundwater flow, but not the local flow at ED-4. Shallow groundwater discharges south to the creek south of the westem portion of ED-4. Groundwater discharges to the wetlands southwest of the eastern portion of ED-4 and aiso south to the creek. Discussion of local groundwater flow should be included in the text

DOE RESPONSE: The bydrogeology discussion has been added to Section 4.3, and local flow has been described in more detail as suggested.

3. Page 6-21. The text states that several metals "were detected at concentrations exceeding $2 \times$ the background concentration, indicating that the observed concentrations of the other metais are not significantly bigher than background." It is not clear from this statement if the remaining metals are actually less than the $2 \times$ backgrownd screening concentrations. The text should be clarified. 
DOE RESPONSE: A sentence has been added to clarify that all other metals are less than the background criteria.

4. Page F.6-1, Section F,6.1, First Bullet. The text says that contaminant concentrations were screened against PRGs "for the residential scenario at risk ievel $1 \mathrm{E}-06$ and HO of $\mathrm{I}$," This contradicts the text in other areas of the Risk Evalualion that specify an Hof of 0 . I. The text should be corrected.

DOE RESPONSE: This is a typographical enror that has been contected. In Section F.6.1 it now states "and HQ of 0.1 ."

5. Page F.6-1, Section F.6.1. The text states, "as discussed in \$ect. F. 1.2, as a back check the results of the screen were evalualed..." This risk evaluation does not contain a Section F. I.2. Further, the basis of the "back check" method is not refereneed in the doeument. The text should be conrected, and additional information regarding the "back check" methoddogy, meluding a citation or reference, should be provided.

DOE RESPONSE: The referenced section should be F.1.1 instead of F.1.2; the text has been revised accordingly. Additional discussion of the risk evaluation methodology has been added to Section F.L.1, and reference to a "back check" has been removed. The text indicates that after the initial screening of data against PRGs, the data were interpreted to evaluate the potential for ELCR $>\mathrm{E}-04$ and/or $\mathrm{HI}>1$. Constituents with delected concentrations above PRGs and background criteria were evaluated in consultation with EPA and with the use of the RAIS risk calculator (http:/rais.ornl.gov/egi-bin/prg/for_ent_data) to confitm that no additional risk calculations were required for ED-4.

6. Page F.6-1, Section F.6.1, Second Set of Bullets. These bullets lay out the procedure that was performed to "back check" the potential risks for constituents that had concentrations that exceeded the PRGs. EPA has itentified several issues with the approach used here.

a. For constituents with a maximum detected concentration that exceeded PRGs, the average concentration was then used in the "back check" method. EPA does not support the use of an average concentration as a surrogate exposure point concentration in a screening method.

DOE RESPONSE: Reference to the "back check" method has been removed. The analysis of constituents defected above PRGs is based on the maximum detected concentration of each constituent to provide a conservative upper-bound evaluation of risks. Based on consultation with EPA and using the RAIS risk calculator (http//tais.oml.gov/cgj-bin/prg/for_ent_data) for confimation, the results of the andysis of maximum detected concentrations indicates that the HI does not exceed 1, and the ELCR is within EPA's generally acceptable range of E-04 to E-06 for ED-4 soil and sediment.

b. The back check method evaluated the average coneentration against an HI of 1 and ELCR of E-04. EPA does not support the use of an ELCR of E-04 as a decision point in a screening method.

DOE RESPONSE: Consistent with the RDR/RAWP (DOE/OR/01-2224\&D3, July 2007), the ELCR is discussed relative to the EPA generally accepted target ELCR range of E-04 to E-06.

c. The back check method used involves dividing the average concentration by the PRG "where appropriate." The text does not specify under what conditions this approach is appropriate. The method used provides a sinple ratio. This may be useful for approximating a Hazard Index, but it 
is not clear how the result could be used to compare against an ELCR of E-04 (or E-06). The back check method appears to be based upon misapplication of guidance described in a Region 4 memorandum from 1994, Amended Guidance for Preltmindry Risk Evaluations (PREs) for the Purpose of Reaching a Finding of Stitability to Lease (FOSL). The PRE method called for use of the maximum detected concentration, which would be divided by the PRG. For carcinogens, the resulting ratio would be multiplied by $1 \times 10^{-6}$ to approximate an $\mathrm{ELCR}$ concentration.

DOE RESPONSE: Reference to the "back check" method has been eliminated. Additional text has been added to provide interpretation of the potential risks and hazards associated with constituents above the PRG, and the text indicates that conclusions are based on consultation with EPA and confirmation using the RAIS risk calculator (http//tais.oml.gov/egibin/prg/for_ent_data). The result of the analysis for ED-4 based on maximum detected concentrations indicates that the HI does not exceed 1, and the ELCR is within EPA's generally acceptable range of $\mathrm{E}-04$ to $\mathrm{E}-06$.

d. The text following the bullets indicates that the HI exceeds 1, but it includes constituents that are present at concentrations below background Metals present at concentrations below their background concentrations should be eliminated from further consideration.

DOE RESPONSE: Metals and radionuclides with maximum detected concentrations below the ETTP background eniteria have been eliminated fiom consideration prior to the estimation of risks.

e. The text following the bullets also indicates that when constituents that are "not site-related" were eliminated, the HI was less than 1. The text does not deseribe how it was delemined that constituents were not site related.

DOE RESPONSE: Text has been modified and the reference to "site-related" constituents has been revised.

7. Page 6-16, Section 6.1.4.1. Discuss the depth of the piezometers.

DOE RESPONSE: Text has been added to Section 6.1.4.1 that notes the depths of the piezometers. Note that the depths of the piezomelers are also given in Table 6.11.

8. General Commenl. The unconsolidated zone well, UNW-125, is not a downgradient well to the ED-4 property. This well is a side gradient well based on topography. Text should be modified to reflect this on page 6-17, $1^{\text {sa }}$ paragraph; page 6-19, last paragraph; page 6-22, last paragraph; and

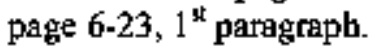

DOE RESPONSE: The description of UNW-125 on pp. 6-17 and 6-19 has been revised from downgradient to side-gradient. However, the references to the piezometers and springs located in ED-3 on pp. 6-22 and 6-23 should remain as downgradient because they are downgradient. 


\section{PROPERTY IDENTIFICATION}

The land referred to as Parcel ED-4, discussed in this Environmental Baseline Survey (EBS), is located in the southeastern portion of the East Tenuessee Technology Park (ETTP) [formerly the Oak Ridge Gaseous Diffusion Plant (ORGDP) and later the K-25 Site] on the Oak Ridge Reservation (ORR) in Roant County, Tennessee. It consists of two noncontiguous tracts comprising a total of approxirnately 18 acres. The wegtern tract of ED-4 encompasses approximately 8.5 acres in the northeastern quadrant of the intersection of Boulevard Road and Highway 58. The eastern tract encompasses ath area of approximately 9.5 acres in the notthwestern quadrant of the intersection of Blair Road and Highway 58. Figure 1.1 is a map showing the relationship of Parcel ED-4 to ETTP. Figure 1.2 shows the footprint of the Parcel ED-4 study area, and Fig. 1.3 is a photo (circa 1945) showing historical activities within a portion of the ED-4 footprint. Visible in this photo are several buildings associated with the Happy Valley Construction Campsite (HVCC), including a Recreation Hall, the Town Hall Camp Operations Building and the Property Warehouse. A railroad spur used during plant construction and the former Wheat Road are also visible.

Highway 58 bounds both tracts of land to the south. The eastern boundary of the eastern tract follows Btair Road for nearly $500 \mathrm{ft}$ from its intersection with Highway 58 to the point at which Blair Road turns eastward. From this point the notthetn boundary trends to the west for approximately 950 ft before turning to the soutb-southeast toward Highway 58 . From the Blair Road and Highway 58 intersection, the southem boundary of the eastern tract runs approximately $1200 \mathrm{ft}$ to the west along Highway 58 . This tract includes a former railroad bed and a segment of the former Wheat Road. The "Haul Road," is a U.S. Department of Energy (DOE)-owned road actively used for truck transport of waste materials to the DOE Environmental Management Waste Management Facility, also bisects this tract (the Haul Road is not a part of the property to be transferred). The western boundary of the western tract is formed by the intersection of Boulevard Road and Highway 58 and runs north for a distance of approximaleiy $600 \mathrm{tt}$ across the comer of a parking lot (following the Zone 2 boundary). The boundary then turns northeast for approximately $300 \mathrm{ft}$ before turning southeastward for approximately $600 \mathrm{ft}$ and then to the south to Highway 58. The southem boundary of the westem tract nuns from the Boulevard Road and Highway 58 intersection to the east for approximately $900 \mathrm{ft}$ along Highway 58. The footprint of Parcel ED-4 is outside of any Environmental Management (EM) Program areas planned for clean-up (DOE 2002).

Preparation of this report included a review of government records, title documents, and aerial protos; visual inspections of the property and adjacent properties; and interviews with current employees ${ }^{2}$ to identify any ateas on the property where hazardous substances and petroleum products or their derivatives were known to have been released, or disposed of.

\footnotetext{
${ }^{2}$ Personal communications with S. T. Goodpastume asd R. P. Prince (currently employed at ETTP).
} 


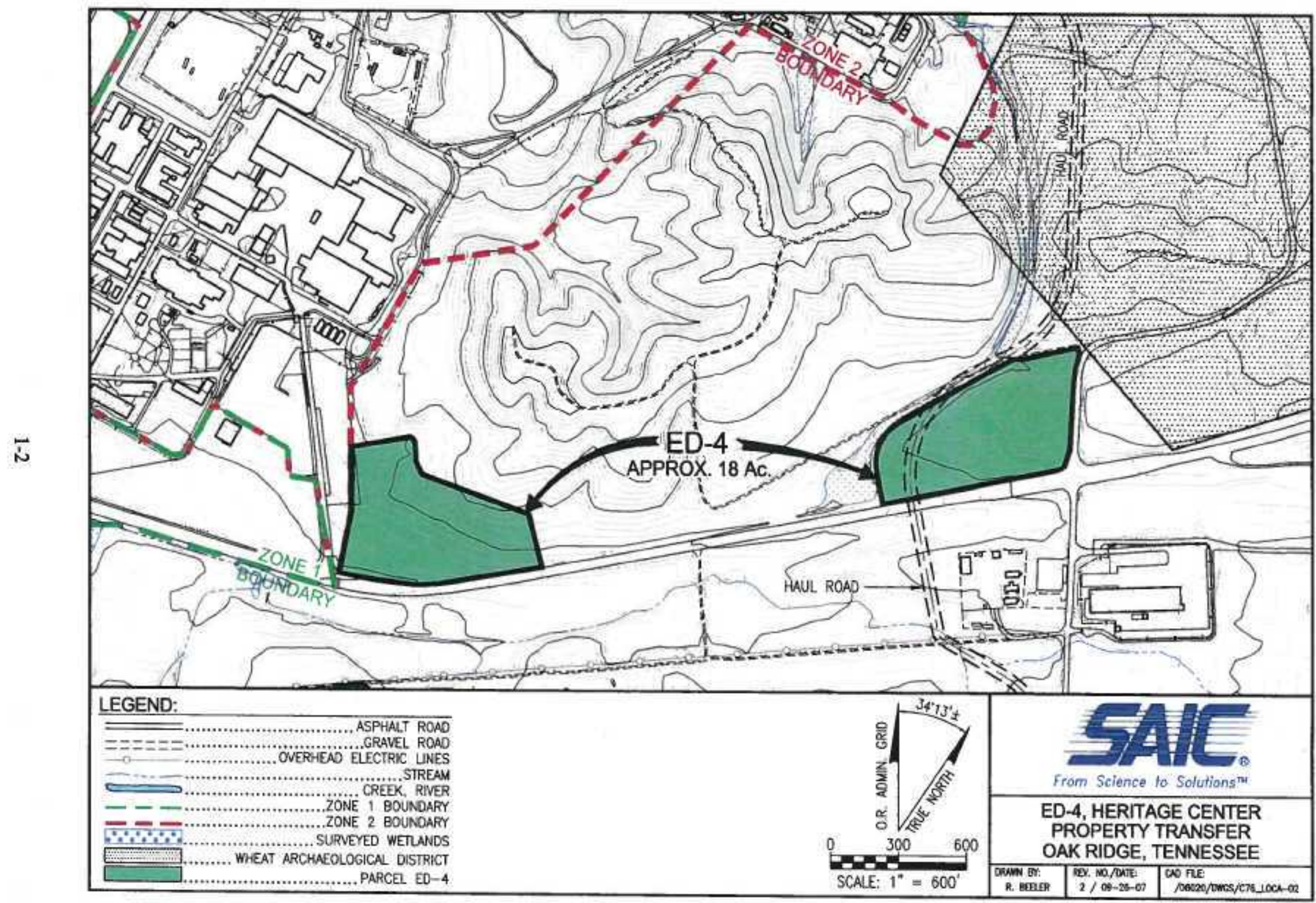

Fig. 1.1. Location map of the Parcel ED-4 study area. 


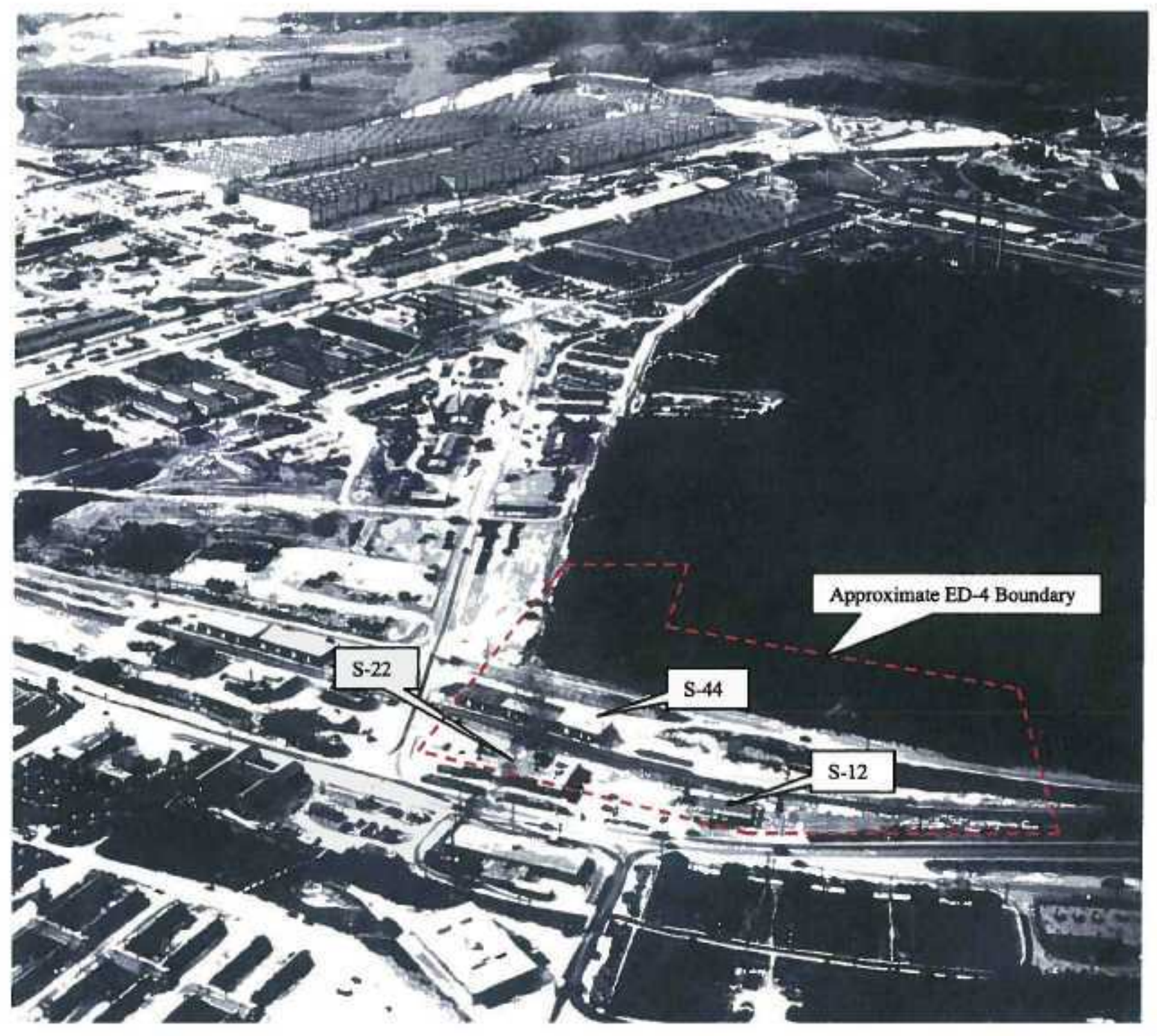

Fig. 1.2. Circa 1945 aerial photo showing historical activities within a portion of the ED-4 footprint. 


\section{TITLE SEARCH}

On July 20, 2007 a visit was made to the state of Tennessee Roane County Registrar's Office to conduct a review of the recorded deeds documenting previous ownership of the land tract where the ED-4 study area is located.

The deeds that conveyed the propenty from the previous owner to the U. S. Govemunent, and any deeds that conveyed the propenty to that previous owner, were reviewed as a part of the titie search. Generally, the deeds from the previous two owners of a particular ORR parcel provide information that goes back to the early 1900 s or even earier. The deeds were reviewed for any references to previous land uses (e.g., homestead, farm, school, business, etc.). Also reviewed were any easements or conveyances referenced in the deeds that might indicate that portions of the land were used for pipelines, potwer lines, etc. Partial disposal or acquisition conveyance deeds were also reviewed because, in some instances, the land comprising a large farm had been acquired via several separate acquisitions.

In addition, property assessment records from the County Property Assessor's Office were reviewed because these docurnents may also contain evidence of a particular land use. Survey or subdivision maps referenced in deeds and maintained in the Register of Deeds office were also reviewed for any indications of a previous land use. Furthermore, because the Tennessee Valley Authority (TVA) was the previous owner of several large tracts of ORR land, the TVA Real Estate Office was contacted regarding their knowiledge of any previous land uses. The U. S. Amy Corp of Engincers (COE) was another source of infornation that has been contacted regarding previous land uses. ${ }^{3}$

\footnotetext{
${ }^{3}$ Energy Systems 1996. Real Estate Section of Comprehersive Environmental Response, Compensation, and Liablity ACs (CERCLA) SECt. I3Oßh) Review, authored by W. W. Teer, Jr., Real Estate Manager, Lockheed Martin Ethergy Systems, lac., Oak Ridge, TN, August 9, 1996.
} 


\section{FEDERAL RECORDS SEARCH AND REGULATORY SUMMARY}

\subsection{FEDERAL RECORDS SEARCH}

The TVA in Knoxville, Tennessee, and the COE District Office in Nashville, Temessee, were contacted in 1996,1997, and again in 1998, to delemine if they maintained any records reflecting past or present land use relative to the land presently comprising ED-4.

Page A-3 of Appendix A is a statement from the Realty Officer of the DOE Oak Ridge Office (ORO) that the real estate records contained no infomation or references to other recorded evidence that, prior to DOE ownership, the property was utilized for the storage of hazardous substances. Additionally, no information contained in these records would indicate that hazardous substances were released from or disposed on the property.

The pre-construction aerial photographs and maps listed below that reflect prior use of this land were aIso reviewed. Copies of these photographs and maps are maintained on file in the DOE-ORO Real Estate Office.

Aerial Photographs:

Photograph Nos. and Date

Nos. 820-3-20 and -21, dated September 25,1942
Flloht By

Aero Service Corp. for Stone and Webster
Source

DOE-ORO, Real Estate Office

These photographs, which were taken in 1942, show that the land on which the study area is located was predominantly used for agricultural purposes. The remaining land was wooded. A map depicting pre-World War $\Pi$ structures, churches, and cemeteries that were present in the area of the current ETTP is also included on page B-3 in Appendix B.

\section{Topographic and real estate maps:}

1. A November 7, 1942, topographic map identified as Section A-2 of ORR that was prepared by Aero Services Corporation for Stone and Webster.

2. A November 1940, Land Acquisition Land Map (sheet $10 \mathrm{~N} 57$ ), prepared by the TVA, shows the boundaries of all land tracts that were acquired for the impoundment of the Watts Bar Reservoir.

3. A February 19. 1945, real estate acquisition map (sheet 9 of 16 ), prepared by the $U$. S. Army, shows the boundaries of the land tracts in Segment $H$ of the ORR that were acquired during the early 1940s for the construction of the ORGDP.

Neither the aforementioned photographs nor maps contained any infomation regarding the history of the past land use that would indicate that releases of hazardous substances or petroleum products or their derivatives have occurred on the land where ED-4 is located. Copies of the 1942 topographic map and real estate map are maintained in the DOE-ORO Real Estate Office. ${ }^{4}$

\footnotetext{
${ }^{1}$ Energy Systems 1996. Real Estate Section of Comprehensive Environmental Response, Compensation, and Liablity Act (CERCLA) Sect. I201hJ Review, authored by W, W. Teer, Jr, Real Estate Manizer, Loekcheed Martin Energy Systems, Inc., Oak Ridge, TN, Augusi 9,1996 .
} 


\subsection{REGULATORY SUMMARY}

As discussed previously, prior to ownership by DOE (and its U. S. Govemment predecessor agencies), the property was farmland and forested land Any DOE operations within the footprint of Parcel ED-4 occused under DOE's own authority, without external regulation, prior to 1984. [DOE became subject to extemal regulations, including the Resource Conservation and Recovery Act of 1976 (RCRA), in 1984].

Based on interviews with tmployees and a review of records, there was no evidence of releases of hazardous substances, or petroleum products or their derivatives, that would preclude identification of Parcel ED-4 as an uncontaminaled parcel.

Records (containing information about spills, permits, or permit violalions) and interviews with employees or fonmer employess ${ }^{5}$ do not indicate that any regulatory aclions bave occured within the footprint of Parcel ED-4. Therefore, no regulatory responses have been invoked.

\footnotetext{
${ }^{5}$ BJC 2007. Personal communications with S. T. Goodpashre ard R. P. Prince (cumently employed at the East Tethnesses Techoology Park) in Augrast,
} 


\section{PAST AND PRESENT ACTIVITIES}

\subsection{PAST AND PRESENT ACTIVITIES FOR THE REAL PROPERTY PROPOSED FOR TRANSFER}

Aerial photographs and site maps from throughout the history of ORGDP indicate that this aren has largely been undeveloped woodland since federal acquisition. Before that time, the land where ED-4 is located consisted primarily of forests and grassiands internixed with large and smali onchards, cropland, and pastures associated with the agriculnurally based and no longer extant Wheat Community. Blacksmithing, brick making, and gristmill operations also occurred in the community.

Workers building the ORGDP lived in a housing area referred to as the Happy Valley Construction Campsite (HVCC), which was located south of Highway 58(outside of ED 4). However, three support facilities for the construction workers were located in the westen tract of ED-4. The three HVCC buildings located within ED-4 were designated as S-12, S-22, and S-44. Historical records indicate that these buildings were the Recreation Hall (S-12), the Town Hall Camp Operations Building (S-22), and the Property Warehouse (S 44). A railroad used during site construction passed through both tracts of ED4, as did a segment of Wheat Road. By 1963 , no remnants of these former structures, including the railroad spur, are visible on aerial photographs.

Historical maps of the ETTP area that were reviewed to deternine whal former DOE facilities bad been located in the ED-4 footprint included the following:

- Kellex Corporation Drawing No. FD-01-AA-02, K-25 and K-27 Plot Plan, Rev. 9, dated March 31, 1946.

In the last two years, DOE bas constructed a Haul Road used for the transport of Comprehensive Environmental Response, Compensation, and Liability Act of 1980 (CERCLA) waste on the Reservation, which cuts across the easten tract. The road is gravel and is elevated above the surrounding terrain. The Hatil Road property is outside of the proposed transfer footprint.

\subsection{PAST AND PRESENT ACTIVITIES FOR THE ADJACENT PROPERTY}

The property to the east and immediately south of Pareel ED-4 has been designated as Parcel ED-3. Parcel ED-3 consists of two noncontiguous land areas, with the much larger area across the Oak Ridge Tumpike from ETTP and south of Parcel ED-4. Prior to federal acquisition, the land in Parcel ED-3 was mostly forest and grassland mixed with orchards, cropland, and pastures. Portions of a small agricuitural community, the Wheat Community, were located on the parcel. Although the primary land use around the Wheat Community was agricultural, the community was a center for religion, education, and trade in eastem Roane County. There is no evidence that activities involying hazardous substances or petroleum products were present in the Wheat Community prior to federal acquisition.

During construction of the ORGDP, the ED-3 tract located south of Highway 58 was a portion of the campsite that provided living quarters from 1943 to 1946 for the construction workers building the ORGDP. The history of the construction camp, known as the HVCC, is described in detail in the Remedial Site Evaluation (RSE) Report (DOE 1997b). 
Currently the only activities conducted at ED-3 occur at the trailers and sheds localed in the northeastem comer of the HVCC tract. These facilities are used as office space and for equipment storage by Restoration Services, lne., in support of ongoing EM activities.

The DOE EM program established the footprint reduction program in the mid-1990s as part of an incremental process to identify ORR lands that have not been impacted by activities that bave resulted in hazardous substance contamination and to issue all such lands a No Further Investigation (NFI) status. The footprint reduction process followed the CERCLA 120(h) process that requires that the following information sources be used to identify the potential presence of hazardous substance contamination on government land: bistorical records, historical aerial photography, and field investigation/verification. As part of the DOE's footprint reduction process, the McKinney Ridge Study Area, which includes an area inmediately east of Parcel ED-4, was evaluated for the purpose of an NFI determination under CERCLA. The evaluation included a review of historical records, aerial photographs, remote sensing data, and ficld investigation/verification. The Tenthessee Department of Environment and Conservation (TDEC) DOE-Oversight Office (DOE-O) and U. S. Environmental Protection Agency (EPA) Region 4 approved the $\mathrm{NFl}$ status for the McKinney Ridge Study Area on May 28, 1998 , and March 10, 1998, respectively.

An NEI determination has been made on the majority of both tracts of land that comprise ED-3. An NFI was approved by the EPA and TDEC in March and April of 1998, respectively, for the HVCC (DOE 1997a), which included the southem ED-3 tract. An NFl for the McKinney Ridge Study Area (DOE 1997a), which included the northem ED-3 tract, with the exception of a 150-ft-wide strip of land along Blair Road and $\mathrm{H}$ jghway 58 , was approved by TDEC and EPA in May and June of 1997, respectively.

The area to the north of ED- 4 was forested prior to acquisition by the federal govemment and has remained forested throughout the history of operations at ETTP. The area to the west of ED-4 encompasses land and building areas that are included in both Zone 1 and Zone 2 of the ETTP. These areas are described in the Phased Constuction Completion Reports (PCCRs) for the K-1007 Ponds Area (DOE 2005a) and the fiscal year 2006 PCCR for Zone 2 (DOE 2006b).

\subsection{HYDROGEOLOGIC ENVIRONMENT}

Pareel ED-4 is located in the southern portion of the ETTP, which is underlain by bedrock of the Chickanauga Supergroup and the Rome Formation (Fig. 4.1). Clastic bedrock of the oider Rome Formation ${ }^{6}$ bas been placed over the ealcareous rocks of the Chickannauga Supergroup by the K-25 thrust fault, which trends generally south to north across the western tract of ED-4 (Fig. 4.1). The Whiteoak Mountain Fault, which trends in a southwest-notheas dirtetion along the southem boundary of Parcel ED-4 is a regional thrust fault that also places rocks of the Rome Formation in contact with rocks of the Chickamanga Supergroup.

The notheastem half of the westem tract and the entire eastern tract are underlain by the Rome Fornation. The lower part of the Rome Formation, which is poorly exposed in the ETTP area, generally consists of thin-bedded shale and siltstone with interbedded sandstones in variegated colors of maroon, green, and yellow-brown. The upper Rome consists of maroon sandstone, siltstone, and shale, In situ weathering of the Rome Formation yields stapolite consisting of weathered shale or siltstone, which commonly becomes more competent with depth. Available exposures of this weathered saprolite in the area north of Pancel ED-4 reveal numerous tight, highly fractured folds with widely ranging bedding orientations. This degree of variability precludes predictions of bedruck flowpaths in the Rome Formation at the ETTP.

\footnotetext{
'P. J. Lerniţيki, I994. Geological Mapping of the Oak Ridge K-25 Site, Oak Ridge, Tennessee, KJER-I l.
} 


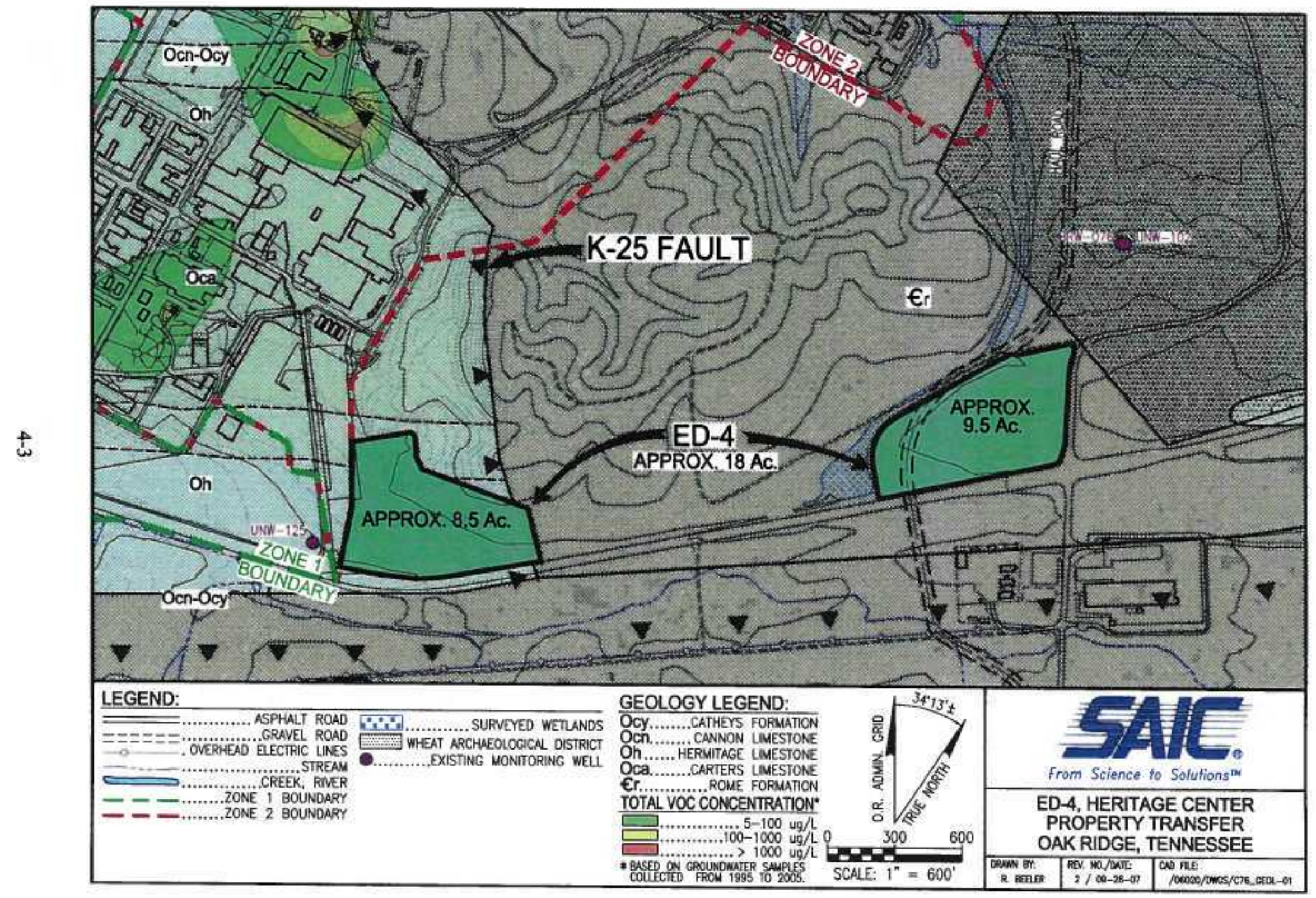

Fig. 4.1. Geologic and topographic map of the ED-4 area. 
The Chickamauga Supergroup formations, which underlie the southwestern comer of the western 1racl of ED-4, include the Carlers Limestone, the Hemitage Fornation, the Cannon Limestone, and the Catheys Formation. Although less prone to karst development than the Knox Group rocks in the vicinity of the ETTP, the Chickamauga formations are nevertheless subject to the development of karst. Solutionally enlarged fractures, joints, and bedding planes are common in exposures of Chickarnauga rocks in the vicinity of ETTP. Structuralty, these formations have been folded into an anticline (convex upward fold) in the vicinity of ParceI ED-4 with the axis of this structure located approximately $600 \mathrm{ft}$ north of the western parcel and trends soutbwest-northeast. Bedding in the Chickanauga generally dips norlhwestwart on the north side of this axis and southeastward on the south side of this axis. The western tract of Parcel ED-4 is located on the south side of the anticline axis; thus, bedding is expecled to dip primarify to the southeast in the vicinity of the tract. However, movement along the Whiteoak Mountain Fault may have caused significant disturbance of bedding orientations in the area of ED- 4 .

Depth to bedrock, interpolated from available data in the general vicinity of ED-4, is expected to be from 12 to $30 \mathrm{ft}$ below ground surface (bgs). The depth to groundwater, interpolated from the available data, is expected to range from $51025 \mathrm{ft}$ bgs depending on lopographic position within the ED-4 parcels. The water table at ETTP generally minics topography with shallow groundwater flowing from higher topographic areas to the surrounding surface water bodies. Groundwater flow through bedrock is primarify controlled by fractures, bedding planes, and hydraulic gradient, and specific flow paths are difficult to discem. Shallow groundwater flow at ED-4 is likely to be to the soulhwest in the western tract of ED-4 with shallow groundwater moving from the bighter topegraphic area in the northeast portion of this tract and discharging to the creek south of Highway 58 . Groundwater flow beneath the eastem tract of ED-4 will be in a radial pattern off of the higher topographic area in the east central portion of this tract and discharging to the wetlands located to the northwest and west of the eastern portion of ED-4, wilh somt flow also to the south toward the lower topographic area on the south side of Highway 58.

A groundwater plume has not been identified beneath or upgradient of ED-4. The nearest groundwater plume is located approximately $700 \mathrm{ft}$ northwest of, and cross-gradient to, ED-4, and occurs in bedrock. The role of the K-25 faut on groundwater movement has not been delemined at ETTP. However, the possibility of transport of contaninated groundwater found cross-gradient and in a different sub-watershed through bedrock flowpaths to ED-4 is not considered to be likely based on hydraulic gradients, which are anticipated to transport the groundwater plume to the southwest away from ED-4. Volatile organic compounds (VOCs) have not been detected at concentrations above a federal drinking water maximum contaminant leve] (MCL) or TDEC domestic water supply criteria ${ }^{3}$ at any of the existing monitoring wells in the vicinity of ED-4. Additjonal information on the hydrogeology of ED-4 can be found in the approved Sampling and Analysis Plan (SAP) [Appendix C].

${ }^{7}$ The Tennessee Department of Environnent and Conservation domestic water supply criteria are tquivalent to the EPA's primary finking waltr maximum contarnimant levels (MCLB) indicated in Table 4.2 with the exception of chloroform, which does not have a corresponding state domestic water quality criterion. 


\section{RESULTS OF VISUAL AND PHYSICAL INSPECTIONS}

\subsection{VISUAL AND PHYSICAL INSPECTIONS OF THE PROPERTY FOR TRANSFER}

A visual inspection of the propesty was conducted on May 3, 2007 and a walkover assessment conducted from May 8 to 14,2007 . At that time, the area was partially grass-covered and partially forested with the exception of the segment of Haul Road that transects the eastem tract of ED-4. The grass-covered areas are routinely mowed. There was no visible evidence of disposal of hazardous substances on or in the vicinity of the road during the walkover assessment. The work instruction for conducting the walkover assessment is presented in Appendix D.

A concrete pad that may be a remnant of a fomer structure associated with the historical railroad spur that crossed the pareel was identified during the walkover assessment. A minor amount of debris (e.g., small pieces of metal) was also present in the area of the pad. No visible evidence of disposal of hazardous substances was observed in this area

A tecision was made during development of the SAP for Parcel ED-4 that sediment accumulation areas, which would represent locations where potential contaminants may accumulate, would be targeted for sample collection to support the Clean Parcel Determination (CPD). Five sediment accumulation areas within Parcel ED-4 were identified during the walkover assessment conducted in May of 2007. These sediment accumulation areas were sampled to determine if surface soils had been impacted by historical activities at Pareel ED-4. These areas were sampled in accordance with the SAP (Appendix C), and the results are described in Chap. 6 of this report The analytical results for the sediment samples were screned against Zone 1 soil remediation levels (RLs) and EPA preliminary remediation goals (PRGs) for evaluation. These sediment accumulation areas represent the accunulation of surface soils that have been transported by runoff from the surrounding areas.

\subsection{VISUAL AND PHYSICAL INSPECTIONS OF ADJACENT PROPERTY}

During the walkdown of May 3, 2007, the properties immediately adjacent to Parcel ED-4 were also visually inspected for signs of current or historical disposal of hazardous substances or petroleum produets or their derivatives.

Thete is no evidence of any historical activities within the small northern tract of ED-3 (located to the east of ED4's eastern tract) other than a small gravel road heading to the east from the intersection of Blair Road and Higbway 58. A remnant of this road is currently gravel covered and used as a vehicle pullmoff from Blair Road The rest of this tract is either grass covered or wooded. The southem tract of ED-3 is largely forested with some grass-covered areas and a gravel-covered road. The Haul Road also transects the eastern end of the southem tract of ED-3.

A TVA power transmission right-of-way (ROW) is located south of Parcel ED-4 and a natural gas line is losated in the area between the two ED 4 land tracts. No bazardous substances were observed in this area at the time of the walkdown. There was no visible evidence of disposal of hazardous substances in this area.

The area immediately west of the westem tract of ED-4 is occupied by a parking lot and an open, grass-covered lawn. The forner \$-21 Happy Valley Service Station was locabed approximately $150 \mathrm{ft}$ west of the westem tratt. This facility was in operation during the late $1940 \mathrm{~s}$. Four underground storage tanks (USTs) were closed in place at this fommer service station in the summer of 2007. Soil sampling 
conducted for the Dynanic Verification \$trategy (DVS) for Zone 1 of the ETTP indicated shallow surface soils had been impacted by a surface release of diesel fuei; however, subsurface soil data do not indicate past leaks from the USTs (DOE 2006a). The risk to an industrial worker from chemical and radiological exposures within the DVS expostue unit encorapassing the former Happy Valley Service Station was found to be within EPA's acceptable risk range (DOE 2006a).

Closure of the Happy Valley Service Station UST site was completed on August 28, 2007, with all actions being approved by the ETTP Remedial Action Core Tean (DOE 2007a), which includes EPA, TDEC, and DOE. The results of the samples were below the TDEC UST initial screening level criteria for soil with commercial use. Concentrations of benzene, toluene, ethylbenzene, and xylenes (BTEX) and metbyI tertiary butyl ether (MTBE) were not detected in any of the soil samples. Although naphthalente was detected at three of the six sample locations, the maximum concentration detected $(0.69 \mathrm{mg} / \mathrm{kg})$ was less than initial screening level criteria ( $403 \mathrm{mg} / \mathrm{kg}$ ). Thus, there is no evidence that bazardous substances, or petroleum products or their derivatives, from the former Happy Valley Service Station bave impacted ED-4.

NFI determinations have been made on properties adjacent to the eastem and southern boundaries of Parcel ED-4. Based on the results of the walkdown, historical records research, and the approved NFIs, there are no indications of potential impacts to ED-4 from adjacent properties. 


\section{SAMPLING RESULTS}

DOE's Reindustrialization Program has worked closely with the EM Program in an effort to realign data collection and analysis approaches to be consistent and to gain efficiencies where possible. To this end, the approach outlined in Chap. 3, "Approach to DVS," of the document titled Remedial Action Work Plan for Dynamic Verification Strategy for Zone I East Tennessee Technology Park Oak Ridge, Tennessee (DVS), DOE/OR/01-2182\&D3 (DOE 2005) is being followed to support transfers within ETTP. Because Parcel ED-4 is Iocated outside of Zone 1, the general DVS approach has been modified to more closely resemble the footprint reduction process implemented by DOE. A process similar to the footprint reduction process was applied to ED-4 through the implementation of a walkover assessment protocol prepaned to support its transfer (BJC 2007). Specifically, the walkover assessment was conducted by making foot traverses across the property and locating areas of potential anthropogenic impacts and sediment accunulation areas for subsequent sample collection. Soil and sediment sarnples were collected for laboratory analysis from the locations identifted during the walkover (see Appendix D). In addition, becausse RLs do not exist for areas outside of Zones 1 and 2 at ETTP, for evaluation purposes, the sampling results have been screened against the RLs established for Zone 1 and also against EPA residential PRGs.

The SAP for Parcel ED-4 (set Appendix C) was written to address groundwater, which was excluded from the NFI for McKinney Ridge (DOE 1997a), and to address potential sediment accumulation areas and soil sampling locations withis Parcel ED-4. The SAP in Appendix C, which was accepted by EPA and TDEC in May 2007 , provides the analytical requirements for groundwater, sodiment, and soil samples obtained from Parcel ED-4.

\section{I CHEMICAL SAMPLING}

Based on discussions with EPA, it has been agreed that the need to collect soil samples to support tite transfer activities will be determined on a case-by-case basis. Factors such as a facility's past operational history, if any, and geographic location will be considered In addition, the history and knowledge of activities on adjacent properties are evaluated to see if those activities could impact the property proposed for transfer.

This section presents and discusses the resutts of the chemical groundwater, sediment, and soil sampling performed in the study area in 2007.

\subsubsection{Data Validation}

During the data validation process, laboratory data were assigned appropriate data validation flags. These flags were as described below:

" $U$ " When the material was analyzed for but not detocted above the level of the associated value.

" $J$ " When the associated value was an estimated quantity (indicating there was cause to question the accuracy or precision of the reported data).

"UJ" When the analyle was analyzed for but not detected above the associated value; however, the reported value was ant estimate and temonsirated a decreased bnowledge of its accuracy or precision. 
" $\mathrm{R}$ " When the analyte value reported was umusable. The integrity of the analyte's identification, accuracy, precision, or semsitivity raised significant questions as to the reality of the information presented.

"=" When the analyte value reported was detected. The integrity of the analyte's identification, accuracy, and precision was validated.

\subsubsection{Chernical Sampllng Results - Sediment}

\section{Data Validation Summary}

Sediment samples were collected from the five sampling locations within the Parcel ED-4 study area (ste Fig. 6.1), with 228 discrett analytes being validated for this project (EPA 1999, 2004). The samples were collected in June and July of 2007 . There were 22 results rejected during validation. Note that estimated values are considered acceptable information for dala interpretalion.

\section{Results}

The chemical sampling results for sediments at Parcel ED-4 are summarized in Table 6.1 by analysis type and were interpreted is the risk screen (see Appendix F). The analylical results for sediment samples colleclect at ED-4 are being treated as soils for the purposes of the data evaluation, as these sediment accunulation areas serve as collection points for surface soils mobilized by runoff from the sumounding area. The sediment data tables foclude the following information:

- the frequency of detection;

- minimum and maximum detected concentrations;

- location(s) at which detected;

- if detected, concentrations that exceed the cIean-up levels established for Zone 1 of the ETTP;

- PRGs for the residential scenario; and

- an indication whether the PRGs were exceeded by the maximum detected concentration.

Tahle 6.1. Pareel ED 4 sediment chemical dnabytical results summory

\begin{tabular}{|c|c|c|c|c|}
\hline & Analysis type & $\begin{array}{l}\text { Frequency of } \\
\text { detections }\end{array}$ & $\begin{array}{l}\text { Mibinaum } \\
\text { concentration } \\
\text { (migkg) }\end{array}$ & $\begin{array}{l}\text { Maximum } \\
\text { concentration } \\
(\mathrm{m} / \mathrm{kq})\end{array}$ \\
\hline Metals & & $133 / 150$ & $3.0 \mathrm{E}-02$ & $3.4 E+04$ \\
\hline PPCB & & $2 / 115$ & $2.6 \mathrm{E}-02$ & $4.1 \mathrm{E}-02$ \\
\hline SVOC & & $37 / 390$ & 4.4E-04 & 2.7E-01 \\
\hline$V O C$ & & $1 \mathrm{I} / 2 \mathrm{BR}$ & $3.9 \mathrm{E}-104$ & 4.0E-01 \\
\hline
\end{tabular}

Datasen from sampling conducted in 2007 includes duplicate analysis collected for quality control.

$\mathrm{mg} / \mathrm{kg}=$ milligtame themical per lejlogram soil.

$\mathrm{PPCB}=$ pesticites and polychlorinated biphenyls.

SYOC - semiyolabile orgaric compound.

VOC = volstile organic compound. 


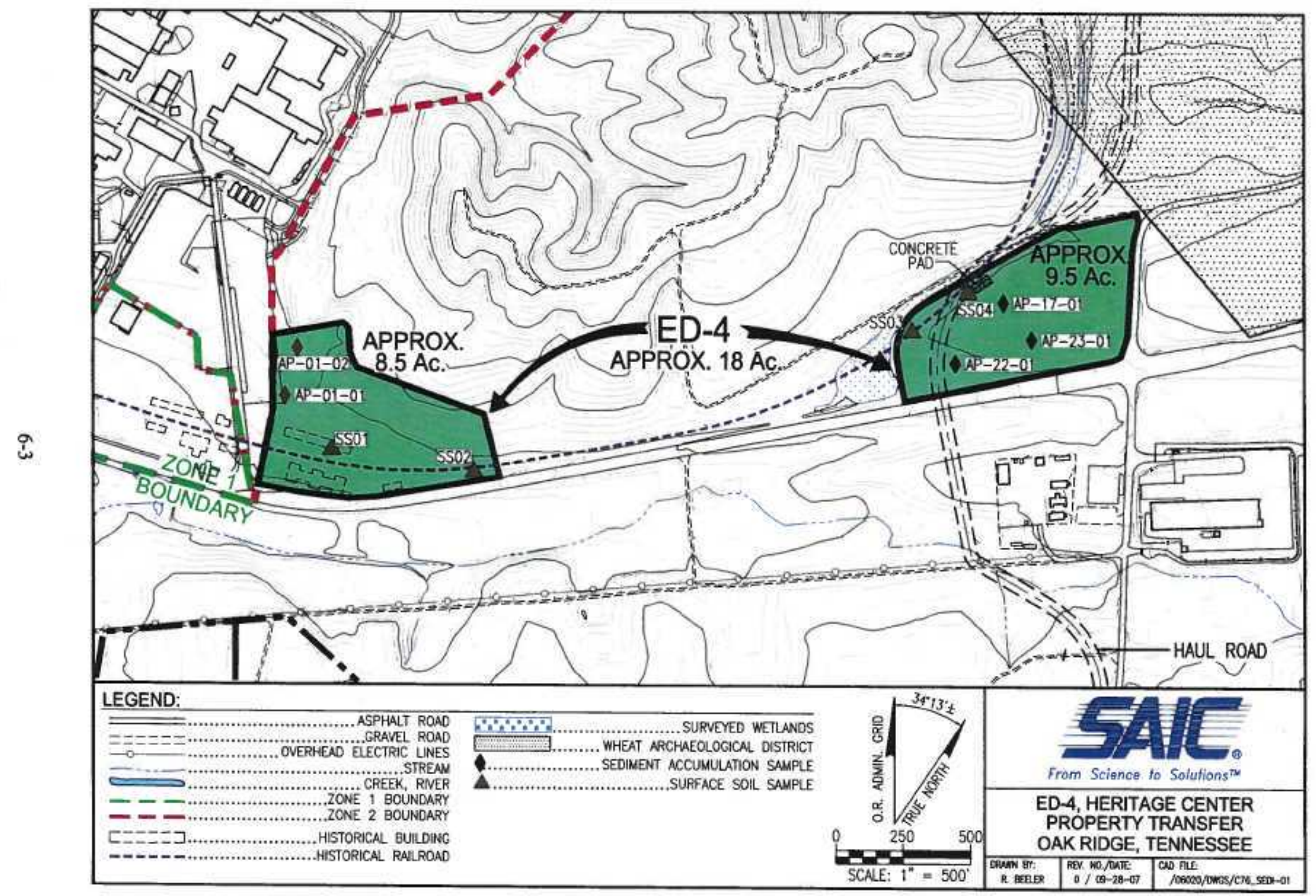

Fig. 6.1. Sediment and surface soil sampling locations at ED-4. 


\subsubsection{Sediment results analysis}

The following discussion summarizes the sediment sampling results for ED-4.

\section{PCBS}

A total of 61 polychlorinated biphenyl (PCB) results were reported (Table 6.2), with 2 tesults (3.3\%) detected. Both PCB-1254 (0.041 mg/kg) and PCB-1260 (0.026 mg/kg) were detected at location AP-01-02. This location receives runoff from a relatively large area of the hillside to the north and east of this location and from the parking lot to the west PCBs bave been used for their heat-resistant properties in oils, paints, and other materials subjected to the effects of high temperature. The detceted PCB results could be the to the accumulation from runoff of small amounts of oils containing PCBs at this location. As presented in Table 6.2, none of the PCB sediment constituents had maximum detected concentrations that exceeded PRGs, indicating a low likelihood of adverse health effects.

Table 6.2. Parcel ED-4 sedlment - polychłorinated biphenyls results

\begin{tabular}{|c|c|c|c|c|c|c|c|}
\hline Angalysis type & $\begin{array}{c}\text { Frequency } \\
\text { of } \\
\text { detections }\end{array}$ & $\begin{array}{c}\text { Minimum } \\
\text { concentration } \\
\text { (mogke) }\end{array}$ & $\begin{array}{c}\text { Maximum } \\
\text { concentratlon } \\
\text { (mo/kg) }\end{array}$ & $\begin{array}{l}\text { Locations } \\
\text { where } \\
\text { detected }\end{array}$ & $\begin{array}{c}\text { Exceeds } \\
\text { Zone 1 } \\
\text { remedistion } \\
\text { levet? }\end{array}$ & $\begin{array}{c}\text { Resideatial } \\
\text { soll } \\
\text { prellminary } \\
\text { remediation } \\
\text { goal } \\
\text { (mg/kg) }\end{array}$ & $\begin{array}{c}\text { Maxignum } \\
\text { defect } \\
\text { exceeds } \\
\text { prelininary } \\
\text { remediatlon } \\
\text { goat } \\
\text { ? }\end{array}$ \\
\hline PCB-J016 & $0 / 4$ & $\mathrm{~N} / \mathrm{A}$ & $\mathrm{N} / \mathrm{A}$ & & $\mathrm{N} / \mathrm{A}$ & $3.9 \mathrm{E}-01$ & $\mathrm{~N} / \mathrm{A}$ \\
\hline PCB-122I & $0 / 6$ & $\mathrm{~N} / \mathrm{A}$ & N/A & & N/A & 1.JE-0] & $\mathrm{N} / \mathrm{A}$ \\
\hline РСB-1232 & $0 / 6$ & $\mathrm{~N} / \mathrm{A}$ & N/A & & $\mathrm{N} / \mathrm{A}$ & $1 . J E-01$ & $\mathrm{~N} / \mathrm{A}$ \\
\hline PCB-1242 & $0 / 6$ & N/A & $N / A$ & & $N / A$ & [.JE-0] & N/A \\
\hline PCB-1248 & $0 / 6$ & $N / A$ & $\mathrm{~N} / \mathrm{A}$ & & $\mathrm{N} / \mathrm{A}$ & J.IE-01 & $\mathrm{N} / \mathrm{A}$ \\
\hline PCB-1254 & $1 / 6$ & $4,1 E-02$ & $4.1 \mathrm{E}-02$ & AP-0] -02 & No & $1.1 E-0\}$ & No \\
\hline PCB-1260 & $1 / 6$ & $2.6 \mathrm{E}-02$ & 2.6E-02 & $A P-01-02$ & No & I. $|E-0|$ & No \\
\hline PCB-1262 & $0 / 6$ & $\mathrm{~N} / \mathrm{A}$ & $\mathrm{N} / \mathrm{A}$ & & $\mathrm{N} / \mathrm{A}$ & $1.1 \mathrm{E}-0 \mathrm{0}$ & N/A \\
\hline PCB-I268 & 066 & N/A & $\mathrm{N} / \mathrm{A}$ & & $\mathrm{N} / \mathrm{A}$ & 1.JE-0] & $\mathrm{N} / \mathrm{A}$ \\
\hline
\end{tabular}

Dataset from 5 ampling eonducted in 2007 inchuies duplicate analysis collected for quality control.

Residential soil PRO at a risk level of $1 E-06$ and a hazard index of 0.1 .

$\mathrm{N} / \mathrm{A}=$ not applicable.

$\mathrm{PCB}=$ polychlorinated biphenyl.

$\mathrm{mg} / \mathrm{kg}$ - fitilligrams chemical per kilograra soil.

VOCs

A total of 288 VOC results were reported (Table 6.3 ), with only 11 results (3.8\%) detected. Constituents were detected at AP-01-01 (1,1-DCE, acetone, 2-butanone); AP-01-02 (acetone, 2-butanone, styrene); AP-17-01 (actone, xylenes); AP-22-01 (xylenes); and AP-23-01 (acelone, toluene) ranging from 0.004 to $0.494 \mathrm{mg} / \mathrm{kg}$ with the higher detections at AP-01-01 and AP-01-02. VOCs are typically used as industrial solvents or components of fuel. The detected VOC results could be the result of the accumulation of soil containing minor amounls of these compounds from releases related to the industrial activities conducted at ETTP. It should be noted that acetone, 2-butanone, and toluene are considered to be comrnon laboratory contaminants. As presented in Table 6.3, none of the VOCs in sediment had maximum detected concentrations that exceeded PRGs, indicating a low likelihowd of adverse health effects from these constituents. 
Table 6.3. Parcel ED-4 sediment - volatile organic compounds results

\begin{tabular}{|c|c|c|c|c|c|c|c|}
\hline Analytis type & $\begin{array}{c}\text { Frequency } \\
\text { of } \\
\text { detectious }\end{array}$ & $\begin{array}{c}\text { Minimum } \\
\text { concentratlon } \\
\text { (wisg) }\end{array}$ & $\begin{array}{l}\text { Maximuni } \\
\text { concentration } \\
\text { (mokg) }\end{array}$ & $\begin{array}{c}\text { Locations } \\
\text { Where } \\
\text { deteeted }\end{array}$ & $\begin{array}{l}\text { Excedts } \\
\text { Zone } 1 \\
\text { remedlation } \\
\text { bval? }\end{array}$ & $\begin{array}{c}\text { Residentil } \\
\text { sol } \\
\text { pretiminary } \\
\text { remediation } \\
\text { gasl } \\
\text { (molkg) }\end{array}$ & $\begin{array}{c}\text { Marintum } \\
\text { detect } \\
\text { erceets } \\
\text { prelimlnosry } \\
\text { remediation } \\
\text { goal } \\
? \\
\end{array}$ \\
\hline 1.I.]-Tichloroethane & $0 / 6$ & $N / A$ & $N / A$ & & $N / A$ & $2.0 \mathrm{E}+02$ & $\mathrm{~N} / \mathbf{A}$ \\
\hline $1,1,2,2-T$ etrachlorwethane & $0 / 6$ & $N / A$ & $N / A$ & & $N / A$ & $4,1 E-01$ & $N / A$ \\
\hline 1,1,2-Tricklomo-1,2,2-trifhromethane & $0 / 6$ & N/A & N/A & & $N / A$ & $2.1 \mathrm{E}+03$ & W/A \\
\hline 1,1,2-Trichortethare & $0 / 6$ & $N / A$ & $\mathrm{~N} / \mathrm{A}$ & & N/A & $7.3 \mathrm{E}-01$ & $\mathrm{~N} / \mathrm{A}$ \\
\hline ],1-Dichlorothane & 046 & N/A & $N / A$ & & N/A & $5.1 \mathrm{E}+01$ & $\mathrm{~N} / \mathrm{A}$ \\
\hline 1,1-Dichlor»etherpe & $1 / 6$ & $1.4 E-03$ & $1,4 \mathrm{E}-\mathrm{aS}$ & $A P-01-01$ & $\mathrm{~N} / \mathrm{A}$ & $12 \mathrm{E}+01$ & No \\
\hline 1,2,3-Trichlowobenzente & 066 & $N / A$ & $N / A$ & & $N / A$ & $N / A$ & $\mathrm{~N} / \mathrm{A}$ \\
\hline 1,2-Dibromo-3-ehloroproppane & 06 & N/A & $N / A$ & & $N / A$ & 2.IE-0I & $N / A$ \\
\hline 1,2-Dibrornoethane & $0 / 6$ & N/A & $\mathrm{N} / \mathrm{A}$ & & $N / A$ & $3.2 \mathrm{E}-02$ & $N / A$ \\
\hline 1,2-Dichloroethane & $0 / 6$ & $N / A$ & N/A & & $N / A$ & $2.8 \mathrm{E}-0 \mathrm{I}$ & $N / A$ \\
\hline 1,2-Dichlorepropane & $0 / 6$ & $N / A$ & $N / A$ & & N/A & $3.4 \mathrm{E}-01$ & $N / A$ \\
\hline 1,2-Dimenthylbenzene & $0 / 6$ & N/A & NA & & $N / A$ & $2.7 \mathrm{E}+01$ & $N / A$ \\
\hline 1,4-Dioxare & Q 6 & NA & $\mathrm{N} / \mathrm{A}$ & & NA & $4.4 \mathrm{E}+0 \mathrm{l}$ & $N / A$ \\
\hline 2-Butanginc & 266 & $2.0 \mathrm{E}-02$ & 3.1E-02 & $\begin{array}{l}\text { AP-01-01 } \\
\text { AP-0।-02 }\end{array}$ & $N / A$ & $2.2 \mathrm{E}+03$ & No \\
\hline 2-Hexanone & $0 / 6$ & $N / A$ & N'A & & $N / A$ & $N / A$ & $N / A$ \\
\hline 2-Methoxy-2-anetbylpropane & $a 6$ & N/A & NiA & & N/A & $1.7 E+01$ & $N^{\prime} A$ \\
\hline 4-Methyl-2-pentanone & ors & N/A & $N / A$ & & $\mathrm{~N} / \mathrm{A}$ & $5.3 E+02$ & $\mathrm{~N} / \mathrm{A}$ \\
\hline A.cetonter & 46 & $5.1 \mathrm{E}-03$ & 4.0E-0I & $\begin{array}{l}A P-01-01 \\
A P-01-02 \\
A P-17-01 \\
A P-23-01\end{array}$ & N/A & $1.4 \mathrm{E}+0.3$ & No \\
\hline Bexzente & 0 & $N / A$ & $\mathrm{~N} / \mathrm{A}$ & & N/A & $6.4 \mathrm{E}-01$ & $N / A$ \\
\hline Bromochloromethane & 06 & N/A & $\mathrm{N} / \mathrm{A}$ & & $N / A$ & $N / A$ & $\mathrm{~N} / \mathrm{A}$ \\
\hline Brotsodichlorsmethane & 016 & $N / A$ & $N / A$ & & N/A & $8.2 \mathrm{E}-[0]$ & $N / A$ \\
\hline Bromoform & $0 / 6$ & N/A & N/A & & $N / A$ & $6.2 \mathrm{E}+01$ & $N / A$ \\
\hline Bnomomethane & $0 / 6$ & $N / A$ & N/A & & $N / A$ & $3.9 \mathrm{E}-01$ & N/A \\
\hline Carton dișulfide & $0 / 6$ & $N A$ & $\mathrm{~N} / \mathbf{A}$ & & $\mathrm{N} / \mathrm{A}$ & $3.6 \mathrm{E}+01$ & N/A \\
\hline Carton letrachloride & $0 / 6$ & N/A & $N / A$ & & N/A & $2.2 \mathrm{E}-0 \mathrm{]}$ & $N / A$ \\
\hline Chlorabentzent & $0 / 6$ & $N / A$ & $N / A$ & & N/A & $1.5 E+0 \mathrm{~L}$ & $N / A$ \\
\hline Chlorothane & $0 / 6$ & N/A & $\mathrm{N} / \mathrm{A}$ & & N/A & $3.0 \mathrm{E}+00$ & $N / A$ \\
\hline Chioroform & $0 / 6$ & $N / A$ & $N / A$ & & N/A & 2.2E-0I & $N / A$ \\
\hline Chlordmethene & $9 / 6$ & $N / A$ & $N / A$ & & $N / A$ & $4.7 \mathrm{E}+0 \mathrm{~d}]$ & $\mathrm{N} / \mathrm{A}$ \\
\hline Cumene & $0 / 6$ & NA & N/A & & $N / A$ & $1.6 \mathrm{E}+01$ & $\mathrm{~N} / \mathrm{A}$ \\
\hline Cyclohexare & 0,6 & $N / A$ & $N / A$ & & $N / A$ & $1.4 E+02$ & $N / A$ \\
\hline Dibromochlonomethane & $0 / 6$ & $N / A$ & NA & & $N / A$ & $1.1 \mathbf{E}+00$ & $N / A$ \\
\hline Dichlomodifuotomethane & 06 & $N / A$ & $N / A$ & & $N / A$ & $9.4 \mathrm{E}+00$ & $\mathrm{~N} / \mathrm{A}$ \\
\hline Ethylbenzene & 06 & N/A & $N_{A}$ & & $\mathrm{~N} / \mathrm{A}$ & $1.9 \mathrm{E}+02$ & $\mathbf{N} / \mathbf{A}$ \\
\hline$M+P X y l e n c$ & $2 / 6$ & $3.95-04$ & $4.6 \mathrm{E}-04$ & $\begin{array}{l}\text { AP-I }-01 \\
\text { AP-22-01 }\end{array}$ & $N / A$ & $2.7 \mathrm{E}+01$ & No \\
\hline Metbyl acelats & 066 & $\mathrm{~N} / \mathrm{A}$ & $\mathrm{N} / \mathrm{A}$ & & $N / A$ & $2.2 \mathrm{E}+03$ & N/A \\
\hline Madbylcyclohexane & $0 / 6$ & N/A & $N_{A}$ & & $N / A$ & $2.6 \mathrm{E}+02$ & $\mathrm{~N} / \mathrm{A}$ \\
\hline Metbylent chloride & 066 & $N / A$ & NA & & $N / A$ & $9.1 E+00$ & N/A \\
\hline Styrente & $1 / 6$ & $4.3 \mathrm{E}-02$ & $4.3 \mathrm{E}-\mathrm{CQ} 2$ & $A^{P}-01-02$ & $N / A$ & $4,4 \mathrm{E}+12$ & $N_{0}$ \\
\hline Tetrachloroethetie & 06 & N/A & $N / A$ & & $N / A$ & 4.8E-01 & $\mathrm{N} / \mathrm{A}$ \\
\hline Toluene & $1 / 6$ & 8.]E-04 & 8.IE-Q4 & $A P-23-01$ & $\mathrm{~N} / \mathrm{A}$ & $6.6 \mathrm{E}+01$ & $N_{0}$ \\
\hline
\end{tabular}


Tabbe 6.3. Parcel ED-4 sediment - volatile organic compounds results (continned)

\begin{tabular}{|c|c|c|c|c|c|c|c|}
\hline Analysis type & $\begin{array}{c}\text { Frequency } \\
\text { of } \\
\text { defections }\end{array}$ & $\begin{array}{c}\text { Minirrurp } \\
\text { concentratton } \\
\text { (mg/kg) }\end{array}$ & $\begin{array}{c}\text { Maximam } \\
\text { conceafration } \\
\text { (mog/kg) }\end{array}$ & $\begin{array}{l}\text { Looptiong } \\
\text { where } \\
\text { detected }\end{array}$ & $\begin{array}{c}\text { Exceeds } \\
\text { Zone } 1 \\
\text { remetintion } \\
\text { level? }\end{array}$ & 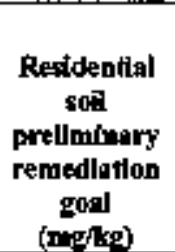 & $\begin{array}{c}\text { Maximum } \\
\text { detect } \\
\text { exceeds } \\
\text { prellminary } \\
\text { remedlation } \\
\text { gosl } \\
?\end{array}$ \\
\hline Thichloroethene & $0 / 6$ & N/A & $\mathrm{N} / \mathrm{A}$ & & N/A & $5.3 E-12$ & $\mathrm{~N} / \mathrm{A}$ \\
\hline Trichlorofluoromethare & $0 / 6$ & N/A & $\mathrm{N} / \mathrm{A}$ & & $N / A$ & $3.9 \mathrm{E}+0]$ & N/A \\
\hline Vinyl chloride & 0,6 & N/A & N/A & & N/A & $7.9 \mathrm{E}-02$ & N/A \\
\hline cis-1, 2-Dithlordethent & 0,6 & $N_{A}$ & $\mathrm{~N} / \mathrm{A}$ & & NA & $4.3 \mathrm{E}+00$ & N/A \\
\hline cis-1,3-Dichloropopene & 06 & W/A & $\mathrm{N} / \mathrm{A}$ & & $N / A$ & $7.8 \mathrm{E}-01$ & N/A \\
\hline trars-1,2-Dichlotoethese & $0 / 6$ & $\mathrm{~N} / \mathrm{A}$ & $\mathrm{N} / \mathrm{A}$ & & NA & $6.9 E+00$ & N/A \\
\hline trans-1,3-Dichloropropene & $0 / 6$ & N/A & N/A & & N/A & 7.8E-01 & N/A \\
\hline
\end{tabular}

Daझaset from sampling conducted in 2007 includes duplicate analysis collected for quality control.

Residenlial soil PRG at a riak level of $1 \mathrm{E}-0 \mathrm{G}$ and a hasand index of 0.1 .

$m 0 / k g=$ millighrams themical per kilogram soil.

$\mathrm{N} / \mathrm{A}=$ not applictable.

\section{SVOCs}

A total of 390 semivolatile organic compound (SVOC) results were reported (Tabile 6.4), with 37 results (10\%) detected. SVOCs are used in various industries and found in lubricants and as components of fuel and asphalt. Detections ranged from $0.0004 \mathrm{mg} / \mathrm{kg}$ for $1,2,4$ trichlorobenzene at AP-02-01 to $0.269 \mathrm{mg} / \mathrm{kg}$ for benzo(a)pyrene at AP-17-01, with detections at ail locations excepl AP-23-01 and generally higher detections found at location AP-17-01. The detected SVOC results copld be the result of runoff from adjoining asphall areas or the use of diesel fuel and/or lubricating oils associaled with activities along the former railroad.

As indicated in Table 6.4, benzo(a)pytene had a maximum detected sediment concentration in excess of the residential soil PRG. The potenlial for adverse health effects associated with concentrations exceeding the PRG is diseussed in the risk evaluation (Appendix F).

Table 6.4. Parcel ED-4 sediment-semivolatile or qanic compaunds results

\begin{tabular}{|c|c|c|c|c|c|c|c|}
\hline Analysta type & $\begin{array}{l}\text { Frequenory } \\
\text { of } \\
\text { detestion: }\end{array}$ & 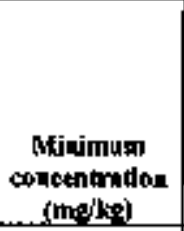 & 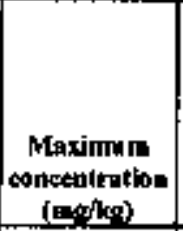 & $\begin{array}{c}\text { Locations } \\
\text { where } \\
\text { detected }\end{array}$ & $\begin{array}{c}\text { Exceds } \\
\text { Zone I } \\
\text { remediatio= } \\
\text { level? } \\
\end{array}$ & $\begin{array}{c}\text { Rtsidential } \\
\text { sol } \\
\text { prelimisary } \\
\text { remedlation } \\
\text { goal } \\
\text { (wig/kg) }\end{array}$ & $\begin{array}{l}\text { Maximum } \\
\text { detect } \\
\text { exceeds } \\
\text { preliminary } \\
\text { renedisting } \\
\text { goul } \\
\text { ? }\end{array}$ \\
\hline I,245-Tetachloroberatese & $0 / 6$ & N/A & N/A & & $N / A$ & $\mathrm{I.8 \textrm {E } + 0 \mathrm { D }}$ & $\mathrm{N} / \mathrm{A}$ \\
\hline 1,2,4-Trichloxobxazente & $2 / 6$ & A. $4 \mathrm{E}-04$ & $4.5 E-04$ & $\begin{array}{l}A P Q I=01 \\
A P-0 \mid=02\end{array}$ & N/A & $6.2 E+00$ & $\mathrm{Na}$ \\
\hline 12-Dichlogobcenzene & $0 / 6$ & N/A & N/A & & $\mathrm{N} / \mathrm{A}$ & $1.1 \mathrm{E}+02$ & $\mathrm{~N} / \mathrm{A}$ \\
\hline 1,3-Dichlorobetizene & 046 & $\mathrm{~N} / \mathrm{A}$ & NiA & & $\mathrm{N} / \mathrm{A}$ & $53 E+01$ & $\mathrm{~N} / \mathrm{A}$ \\
\hline 1,4-Dkehilonotenzone & 046 & N/A. & $N / A$ & & $\mathrm{~N} / \mathrm{A}$ & $3.4 \mathrm{E}+00$ & $\mathrm{~N} / \mathrm{A}$ \\
\hline 2,3,4,6-Tctrachy forophenol & $0 / 6$. & $\mathrm{N} / \mathrm{A}$ & $\mathrm{N} / \mathrm{A}$ & & $\mathrm{N} / \mathrm{A}$ & $1.3 \mathrm{E}+\mathrm{q}$ & $N / A$ \\
\hline $2,4,5-$ Trichlorophenol & 0.8 & $\mathrm{~N} / \mathrm{A}$ & $\mathrm{N} / \mathrm{A}$ & & NA & 6.IE+02 & NA \\
\hline $2,4,6$-Trithilatepthenol & $0, \sqrt{6}$ & $N / A$ & $\mathrm{~N} / \mathrm{A}$ & & $N / A$ & 6.1E0! & $N / A$ \\
\hline 24-Dithloropherial & 06 & $\mathrm{~N} / \mathrm{A}$ & $\mathrm{N} / \mathrm{A}$ & & N/A & $1.9 \mathrm{E}+0 \mathrm{O}$ & $N / A$ \\
\hline
\end{tabular}


Table 6.4. Pareel ED-4 sediment - semivolatle organíc compounds results (continued)

\begin{tabular}{|c|c|c|c|c|c|c|c|}
\hline Analysis type & $\begin{array}{c}\text { Frequency } \\
\text { of } \\
\text { defectlons }\end{array}$ & $\begin{array}{l}\text { Mindnum } \\
\text { concentration } \\
\text { (mafkg) }\end{array}$ & $\begin{array}{l}\text { Maximan } \\
\text { cancentration } \\
\text { (mollkg) }\end{array}$ & $\begin{array}{c}\text { Lotatlons } \\
\text { where } \\
\text { deterted }\end{array}$ & $\begin{array}{c}\text { Exceeds } \\
\text { Zone 1 } \\
\text { remedfaton } \\
\text { level? }\end{array}$ & $\begin{array}{c}\text { Residential } \\
\text { soil } \\
\text { predlinlnary } \\
\text { remedlalinn } \\
\text { Gal } \\
\text { (mg/kg) }\end{array}$ & $\begin{array}{l}\text { Mayinam } \\
\text { detect } \\
\text { exceents } \\
\text { prctbininary } \\
\text { reroediation } \\
\text { gon } \\
?\end{array}$ \\
\hline 2,4-Ditnethylpbenol & Q/6 & $N N A$ & N/A & & $N / A$ & $12 \mathrm{E}+42$ & $\mathrm{~N} / \mathrm{A}$ \\
\hline 2,4-Dinitrophenol & Q/6. & $\mathrm{N} / \mathrm{A}$ & $\mathrm{N} / \mathrm{A}$ & & $\mathrm{N} / \mathrm{A}$ & $1.2 E+01$ & $\sqrt{\mathrm{V} A}$ \\
\hline 2,4-Dinitrotoluene & a.6 & $\mathrm{N} / \mathrm{A}$ & $\mathrm{NA}$ & & $\mathrm{N} / \mathrm{A}$ & $7.2 \mathrm{E}-01$ & $N / A$ \\
\hline 2,6-Dinituotolutet & 016 & N/A & $N A$ & & $N / A$ & $7.2 E-01$ & N/A \\
\hline 2-Chlorornaphthalene & Wh & N/A. & $\mathrm{N} / \mathrm{A}$ & & $\mathrm{N} / \mathrm{A}$ & $4.9 \mathrm{E}+02$ & $\mathbf{N} / \mathrm{A}$ \\
\hline 2 -Chlorophenel & 0,6 & N/A & $\mathbf{N} / \boldsymbol{A}$ & & $N / A$ & $6.3 \mathrm{E}+00$ & $\overrightarrow{N A R}$ \\
\hline 2-Mlethyl-4,6-diniogopher & $0 / 6$ & $\mathrm{~N} / \mathrm{A}$ & $N / A$ & & $N / A$ & 6. $|E-0|$ & $N / t_{2}$ \\
\hline 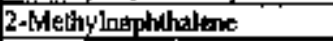 & 016 & $\mathrm{~N} / \mathrm{A}$ & NiA & & N/A & $5.6 \mathrm{E}+00$ & $N / A$ \\
\hline 2-Methylphenol & Ji' & NiA & $N / A$ & & $N / A$ & $3.1 \mathbf{E}+(12$ & $\mathrm{N} / \mathrm{A}$ \\
\hline 2-Nilroberzensamine & $00^{\prime} 6$ & NA & NA & & $\mathrm{N} / \mathrm{A}$ & 1. $8 \mathrm{E}+\dot{+1}$ & $\mathbf{N} / \mathrm{A}$ \\
\hline 2-Nitrophenol & 006 & $\mathrm{Wh}$ & $\mathrm{N} / \mathrm{A}$ & & $N / A$ & N/A & $\mathrm{N} / \mathrm{A}$ \\
\hline 3-Ninobenzetominis & 06 & $\mathrm{~N} / \mathrm{A}$ & $N / A$ & & $N / A$ & 1.8E+00 & NiA \\
\hline 4-Bromopheny phanyl ether & $0 / 6$ & $\mathrm{~N} / \mathrm{A}$ & $N / A$ & & $\mathrm{~N} / \mathrm{A}$ & $\mathrm{N} / \mathrm{A}$ & $\mathbf{N} / \mathbf{A}$ \\
\hline 4Chloro-3-rTethylphenol & $0 / 6$ & $\mathrm{~N} / \mathrm{A}$ & $\mathrm{N} / \mathrm{A}$ & & $\mathrm{N} / \mathrm{A}$ & $\mathrm{Nh}$ & $N / A$ \\
\hline 4-Chlorobetedinninte & $0 / 6$ & $\mathrm{~N} / \mathrm{A}$ & $\mathrm{N} / \mathrm{A}$ & & $N / A$ & $2.4 \mathrm{E}+\mathrm{DI}$ & N/A \\
\hline 4-Chlotophenyl plyeryl eflert & $0 / 6$ & $\mathrm{NA}$ & N/A & & $N / A$ & $N / A$ & NIA \\
\hline 4-Nitrobtarenérjinc & 016 & NA & $N / A$ & & $N / A$ & $1.8 E+01$ & Nidh \\
\hline 4-Nitrophermol & 016 & $N / A$ & Nidh & & Nih & NiA & Ni/h \\
\hline Acensphthetge & $0 / 6$ & NA & M/dA & & $\sqrt{\mathrm{A}}$ & 3. $3 E+02$ & $\mathrm{~N} / \mathrm{A}$ \\
\hline Accliaphthylens & $0 / 6$ & $N A$ & $\mathrm{~N} / \mathrm{A}$ & & NA & 3.JE+CQ & $\mathrm{N} / \mathrm{A}$ \\
\hline Actophrotont & $0 / 6$ & NA & N/A & & $N / A$ & $\mathrm{~N} / \mathrm{A}$ & $\mathrm{N} / \mathrm{A}$ \\
\hline Anthracede & $2 \sqrt{6}$ & $9.9 E_{-13}$ & $1.5 \mathrm{E}-02$ & $A P-|T=0|$ & $N_{A}$ & $2.2 \mathrm{E}+03$ & $\mathrm{No}$ \\
\hline 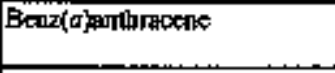 & $3 / 6$ & $2.8 \mathrm{E}-02$ & I.IE-01 & $\begin{array}{l}A P=1701 \\
A P-22-01\end{array}$ & NA & $6.2 E 001$ & No \\
\hline Betlzota)pyrene & $4 / 6$ & $1.4 \mathrm{E}-0 \mathrm{~L}$ & $2.7 E-0]$ & $\begin{array}{l}A P-01-02 \\
A P-17-41 \\
A P-22-01\end{array}$ & N/A & $6.2 E-02$ & Y昰 \\
\hline Benzo(b)flixorandhene & $2 / 6$ & $1.9 \mathrm{E}-01$ & $2.4 \mathrm{E}-\mathrm{Q} 1$ & $\begin{array}{l}A P \cdot 01<02 \\
A P-22-01\end{array}$ & $\mathrm{NAB}$ & $6.2 \mathrm{E} 01$ & No \\
\hline Benzolg,h,tjperylenes & $\overline{36}$ & $7.5 E-02$ & I. $7 \mathrm{E}-0 \mathrm{H]}$ & $\begin{array}{l}A P-0]-02 \\
A P-17-01\end{array}$ & NA & $2.3 \mathrm{E}+42$ & No \\
\hline Berrec(k)fluxorantfiere & 3.46 & $4.2 \mathrm{E}-102$ & $1.2 \mathrm{E}-\mathrm{DI}$ & $\begin{array}{l}\text { AP-01-02 } \\
\text { AP- } 17-01\end{array}$ & WA & $62 \overline{2 E}+00$ & No \\
\hline Bis(2-Chlornethoxy)methame & ors & $\mathbf{N} / \mathrm{A}$ & $N /$ & & $N / A$ & $N / A$ & $\mathbf{N} / \mathrm{A}$ \\
\hline Bis (2-chloroctuyl) elhar & 046 & $\mathrm{~N} / \mathrm{A}$ & $\mathrm{N} / \mathrm{A}$ & & $\mathrm{N} / \mathrm{A}$ & $22 \mathrm{E}-01$ & $\mathrm{~N} / \mathrm{A}$ \\
\hline 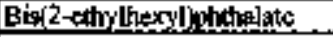 & 016 & $N / A$ & $\mathrm{~N} / \mathrm{A}_{2}$ & & $\mathrm{~N} / \mathrm{A}$ & $3.5 \mathbf{E}+01$ & $\mathrm{NA}$ \\
\hline Buby benzyl hhthalate & 046 & $\mathrm{~N} / \mathrm{A}$ & $\mathrm{NA}$ & & $N / A$ & $12 \mathrm{E}+03$ & N/A \\
\hline Catberole & Q46 & $\mathrm{N} / \mathrm{A}$ & N/A & & $N /{ }^{\prime} A$ & $2,4 E+01$ & NA \\
\hline Clarysene & 46 & $1.95-02$ & $1.7 \mathrm{E}-4 \mathrm{I}$ & $\begin{array}{l}A P-01-02 \\
A P-17-01 \\
A P-22-01\end{array}$ & $N / A$ & $6.2 \mathrm{E}+01$ & No \\
\hline Di-ributyd phthalate & 016 & $\mathrm{~N} / \mathrm{A}$ & $\mathrm{N} / \mathrm{A}$ & & N/A & $6.1 \mathbf{E}+02$ & $\mathbf{N} / \mathbf{A}$ \\
\hline Di-n-octylphuhajate & $9 / 6$ & $N A$ & $N / h$ & & $\mathrm{~N} / \mathrm{A}$ & $2.4 \mathrm{E}+02$ & $\mathrm{~N} / \mathrm{A}$ \\
\hline 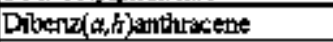 & 066 & $\mathrm{~N} / \mathrm{h}$ & $\mathrm{N}_{\mathrm{A}}$ & & $N / A$ & $62 \mathrm{E}-02$ & N/A \\
\hline Dibetizofirm & 0.6 & $\overline{N / A}$ & $\mathrm{~N} / \mathrm{A}$ & & $\mathrm{N} / \mathrm{A}$ & $1.5 \mathrm{E}+01$ & $\mathrm{~N} / \mathrm{A}$ \\
\hline Dituthyl phthalate & 0,6 & $N / 4$ & $\mathrm{~N} / \mathrm{A}$ & & $\mathrm{N} / \mathrm{A}$ & $4.9 E+03$ & N/h \\
\hline Dimethyl phobalale & $0 / 6$ & $\mathrm{NA}$ & $N / A$ & & N/A & $6.1 E+04$ & $\mathrm{~N} / \mathrm{A}$ \\
\hline Diphęuylintine & 016 & $\mathrm{~N} / \mathrm{A}$ & NA & & $\mathrm{N} / \mathrm{A}$ & $1.5 E+02$ & NiA \\
\hline Fluortithernt & $4 / 6$ & 2.7E-02 & $12 \mathrm{E}-01$ & $\begin{array}{l}A P-01=02 \\
A P-17-01 \\
A P-22-01\end{array}$ & $\mathrm{~N} / \mathrm{A}$ & $2.35+02$ & No \\
\hline Fluprone & $0 \sqrt{6}$ & $\mathrm{NA}$ & $N / A$ & & NiA & $2.7 \mathrm{E}+02$ & $\mathrm{NA}$ \\
\hline Hexachlorobernene & 016 & $\mathrm{~N} / \mathrm{A}$ & N/A & & Nis & $3.0 \mathrm{E}-01$ & $\mathrm{Wh}$ \\
\hline
\end{tabular}


Table 6.4. Parcel ED-4 sediment - semivolatite organic compounds results (cintinued)

\begin{tabular}{|c|c|c|c|c|c|c|c|}
\hline Analyts typt & $\begin{array}{c}\text { Frequency } \\
\text { of } \\
\text { deteclions }\end{array}$ & $\begin{array}{c}\text { Minlmum } \\
\text { contentration } \\
\text { (ingtikg) }\end{array}$ & $\begin{array}{c}\text { Maximmm } \\
\text { contentration } \\
(\text { moj/kg) }\end{array}$ & $\begin{array}{c}\text { Lacations } \\
\text { where } \\
\text { detected }\end{array}$ & $\begin{array}{c}\text { Exceds } \\
\text { Zont } 1 \\
\text { remertistion } \\
\text { level? }\end{array}$ & $\begin{array}{l}\text { Aesidentinl } \\
\text { sot } \\
\text { prelimbitery } \\
\text { remediation } \\
\text { goal } \\
\text { (mglkg) }\end{array}$ & $\begin{array}{c}\text { Maxireum } \\
\text { detect } \\
\text { efcteds } \\
\text { preliminary } \\
\text { rementiation } \\
\text { gotl } \\
?\end{array}$ \\
\hline Hexactiboubuladidient & 016 & N/A & $N / A$ & & $\mathrm{~N} / \mathrm{A}$ & $1.8 \mathrm{E}+00$ & $N / A$ \\
\hline Hexactilondyclopendadiente & Ads & N/A & $N / A$ & & $\mathrm{~N} / \mathrm{A}$ & 3. $7 \mathbf{E}+0.1$ & $\overline{N / A}$ \\
\hline Hexachlorkethape & $0 / 6$ & $\mathrm{~N} / \mathrm{R}$ & $\mathrm{N} / \mathrm{A}$ & & $\mathrm{N} / \mathrm{A}$ & $6, \mid E+90$, & N/A \\
\hline 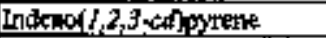 & $2 / 6$ & 5.7E-02 & $1.2 \mathrm{E}-0.1$ & AP-17-01 & NiA & $6.2 \mathrm{E}=01$ & $\$ \omega_{0}$ \\
\hline Isophorone & $0 \times 6$ & $\mathrm{NUA}$ & $\mathrm{N} / \mathrm{A}$ & & $N / A$ & $5.1 \mathrm{E}+02$ & $\mathrm{~N} / \mathrm{A}$ \\
\hline N-Nitrosom-di-n-pupy iamine & $0 \%$ & $\mathbf{N} / \mathbf{A}$ & $\mathrm{N} / \mathrm{A}$ & & $\mathrm{N} / \mathrm{A}$ & $6.9 \mathrm{E}-02$ & $\mathrm{~N} / \mathrm{A}$ \\
\hline Neapthis ene & $0 / 6$ & $\mathrm{~N} / \mathrm{A}$ & $\mathrm{N} / \mathrm{A}$ & & N/A & $5.8 \mathrm{E}+00$ & NA \\
\hline Nitrobyorzente & $0 / 6$ & $\mathrm{~N} / \mathrm{A}$ & N/A & & $\mathrm{N} / \mathrm{A}$ & $20 \mathrm{E}+\mathrm{d} 0$ & $\mathrm{~N} / \mathrm{A}$ \\
\hline Penlagchlorcphenol & a/6 & $\mathrm{N} / \mathrm{A}$ & $\mathrm{N} / \mathrm{A}$ & & $\mathrm{N} / \mathrm{A}$ & $3.0 \mathrm{E}+00$ & $\mathrm{~N} / \mathrm{A}$ \\
\hline Phenombitente & 316 & $2.2 \mathrm{E}-02$ & $2.6 \mathrm{E}-02$ & $\begin{array}{l}A P-01-02 \\
A P-17-01\end{array}$ & $N_{A}$ & $2.3 E+02$ & No \\
\hline Pbtong & $0 \mathrm{y} 6$ & $N / A$ & $N / A$ & & $\sqrt[N]{/ A}$ & $1.8 E+0.3$ & $\mathrm{~N} / \mathrm{A}$ \\
\hline Pyтte & $5 / 6$ & $1.5 \mathrm{E}-02$ & $1.8 \mathrm{E}-91$ & $\begin{array}{l}A[\vec{P}-0]-01 \\
A P-0]-02 \\
A P \cdot 17-01 \\
A P \cdot 2 Z=01\end{array}$ & $\mathrm{~N} / \mathrm{A}$ & $23 \mathrm{E}+02$ & No \\
\hline Ë+phetryiphenol & 06 & $N / A$ & $\mathrm{~N} / \mathrm{A}$ & & $\mathrm{N} / \mathrm{A}$ & $\mathrm{N} / \mathrm{A}$ & $N / A$ \\
\hline
\end{tabular}

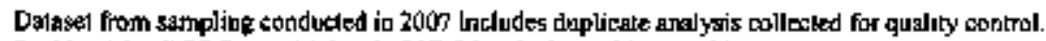
Regidential soil PRG at a rigk level of $1 \mathrm{E}-06$ and a haterd iadex of 0.1 .

mpikg - molbiguans shertical per bilogran soil.

NA = oot awailable.

\section{Pesticides}

A total of 61 pesticide results were reported, with no detections.

\section{Herbicides}

A total of 7 herbicide results were reported, with no detections

\section{Mefals}

A total of 150 metals results were reported, with 133 results $(90 \%)$ detected. The detected concentrations ranged from $0.03 \mathrm{mg} / \mathrm{kg}$ for mercury at AP-17-01 to $34,100 \mathrm{mg} / \mathrm{kg}$ for iron at AP-01-01 (Table 6.5). All sample locations were found to have detected metals results, which is to be expected because soil generally has a measurable content of metals in nature. There is no evident contation between sample location and elevated metals results. The highest metals results were for the macronutrient elements calcium, iron, manganeșt, magnesiurn, aluninum, and potassium, which are ali naturally occurring. As indicated in Table 6.5, several of the metals had a maximum detected sediment conentration in excess of the residential soil PRG. Howwever, the established ETTP background soil concentrations for six of these eight metals are bigher than the PRG, and only the maximum concentrations of selenitur and thallium exceed both the PRG and background concentrations. The potential for adverse health effects associated with concentrations exceding the PRG is discussed in the risk evaluation (Appendix F). 
Table 6.5. Parcel ED-4 study area sediment - metals results

\begin{tabular}{|c|c|c|c|c|c|c|c|c|c|}
\hline Anabysis type & $\begin{array}{c}\text { Frequency } \\
\text { of } \\
\text { detections }\end{array}$ & 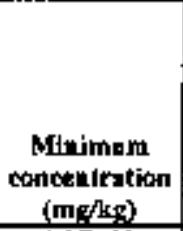 & $\begin{array}{c}\text { Maximum } \\
\text { concentration } \\
\text { (no } / \mathrm{kg})\end{array}$ & Locat & ons whered & fected & $\begin{array}{c}\text { Exceedts } \\
\text { Zone } 1 \\
\text { remedlation } \\
\text { lexd? }\end{array}$ & $\begin{array}{c}\text { Residential } \\
\text { soli } \\
\text { preliminary } \\
\text { remediat|on } \\
\text { goal } \\
\text { (malke) }\end{array}$ & $\begin{array}{c}\text { Maximurs } \\
\text { detect } \\
\text { exceeds } \\
\text { preliminary } \\
\text { kemedlation } \\
\text { goul } \\
?\end{array}$ \\
\hline Alutuintuth & 66 & $3.9 E+13$ & $2.6 \mathrm{E}+04$ & \begin{tabular}{|l|l|}
$A P+01-01$ \\
$A P-22$
\end{tabular} & $\begin{array}{l}A P-01-02 \\
A P-23-01\end{array}$ & $A P=17-[1]$ & $N / A$ & $7.6 \mathrm{E}+03$ & Yes \\
\hline Antimour & $1 / 6$ & 1.TE+00 & I.7E+00 & AP.23.QI & & & $\mathrm{N} / \mathrm{A}$ & $3.1 E+(n)$ & $\mathrm{No}$ \\
\hline Arșenic & $6 / 6$ & $1 . \overline{5}+00$ & $|.3 \mathrm{E}+0|$ & $\begin{array}{l}A P-01-01 \\
A P-22-01\end{array}$ & $\begin{array}{l}\text { AP } 401-02 \\
\text { AP-25-01 }\end{array}$ & $A P-17-0 \mid$ & Na & $3.9 \mathrm{E}-01$ & Yes \\
\hline Esinm & 616 & $2.6 \mathrm{E}+[01$ & $9.4 E+01$ & $\begin{array}{l}\mathrm{AP}-01-0 \mathrm{~L} \\
\mathrm{AP}-22-01\end{array}$ & $\begin{array}{l}A P-0]-02 \\
A P-23-0]\end{array}$ & $A P-17-01$ & NA & $5.4 E+62$ & No \\
\hline Beryllium & 616 & 3.3E-01 & $1.6 E+00$ & $\begin{array}{l}A P-01-01 \\
A P \cdot 22-01\end{array}$ & $\begin{array}{l}A P-0]-02 \\
A P-23-101\end{array}$ & $A^{P}-17-0 \mid$ & No & $1 S E+01$ & $\mathrm{No}$ \\
\hline Beton & $5 / 6$ & $2.4 \mathrm{E}+00^{\circ}$ & $4.6 E+00$ & $\begin{array}{l}A P-0]-[1] \\
A P \cdot 23-0\}]\end{array}$ & $A P-01-12$ & $A P=17-0 I$ & $N / A$ & $1.6 \mathrm{E}+03$ & $N$ \\
\hline Cudmivm & 0,6 & N/A & $N / A$ & & & & N/A & $3.7 \mathrm{E}+00$ & $\mathrm{~N} / \mathrm{A}$ \\
\hline Cakium & $6 / 6$ & $25 \mathrm{E}+03$ & $1.5 \mathrm{E}+04$ & $\begin{array}{l}\text { AP-O1-01 } \\
\text { AP-22-01] }\end{array}$ & $\begin{array}{l}\text { APQ01-102 } \\
\text { AP. } 23-01\end{array}$ & AP:] $7-0 \mid$ & $\mathrm{M} / \mathrm{A}$ & $\mathrm{N} / \mathrm{A}$ & $\mathrm{N} / \mathrm{h}$ \\
\hline Chrominam & $6 / 6$ & $3.3 \mathrm{E}+00$ & $3.1 \mathrm{E}+0]$ & 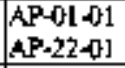 & $\begin{array}{l}\text { AP.01-02 } \\
\text { AP.23-01 }\end{array}$ & $A P \cdot 17 \cdot 01$ & $N / A$ & $2.2 \mathrm{E}+01$ & Yष्ठ \\
\hline Cotaglit & $6 / 6$ & $1.6 \mathrm{E}+00$ & 2.IE+01 & $\begin{array}{l}A P-01-01 \\
A P-22-01\end{array}$ & $\begin{array}{l}\text { AP-01-02 } \\
\text { AP-23-01 }\end{array}$ & $\mathrm{AP}-\mathbf{I}-\mathrm{O}-1$ & $\mathrm{~N} / \mathrm{A}$ & $1.4 \mathrm{E}+02$ & No \\
\hline Copper & 616 & $5.7 \mathrm{E}+00$ & 2.7E+01 & $\begin{array}{c}A+0-0] \\
A P-22-0]\end{array}$ & $\begin{array}{l}\text { AP-0I-02 } \\
\text { AP-23-0| }\end{array}$ & AP-I7-0] & $\mathrm{N} / \mathrm{A}$ & $3.1 E+02$ & No \\
\hline tront & $6 / 6$ & $1.3 E+04$ & $3.4 E+04$ & $\begin{array}{l}A P-[0]-0] \\
A P-22-01\end{array}$ & $\begin{array}{l}\text { AP-01-02 } \\
\text { AP-23.01 }\end{array}$ & AP-I7-0] & NiA & $2.3 \mathrm{E}+03$ & Yes \\
\hline Lead & $6 / 6$ & $60 \mathrm{E}+00$ & $5.2 E+01$ & $\begin{array}{l}A P-01-0] \\
A P-22-\theta 1\end{array}$ & $\begin{array}{l}\text { AP- }-0 \mid-02 \\
\text { AP.23-01 }\end{array}$ & $A P \cdot 17=01$ & $\mathrm{~N} / A$ & $4.0 \mathrm{E}+02$ & No \\
\hline Maguesium & $6 / 6$ & $4.0 E+02$ & $1.0 \mathrm{E}+04$ & $\begin{array}{l}A P+01-01 \\
A P-22-0 \mid\end{array}$ & $\begin{array}{l}\text { AP-01 } 102 \\
\text { AP-2\}-01 }\end{array}$ & $A P=|T-|||$ & $\bar{N} / \mathrm{A}$ & $\mathrm{N} / \mathrm{A}$ & $N / A$ \\
\hline Mengenneșt & $6 / 6$ & $6.1 \mathrm{E}+\mathrm{OI}$ & $1.3 \mathrm{E}+03$ & $\begin{array}{l}A P=01-01 \\
A P=22-01\end{array}$ & $\begin{array}{l}A P-01+02 \\
A P-2\}=01\end{array}$ & $A P-17-0 \mid$ & $N^{\prime} A$ & i. $8 \mathrm{E}+02$ & Ves \\
\hline Maxury & $6 / 6$ & $3.0 \mathrm{E}-62$ & $|-4 \mathrm{E} \cdot 0|$ & $\begin{array}{l}A P-01-01 \\
A P-22-01\end{array}$ & $\begin{array}{l}A P-01-02 \\
A P-23-01\end{array}$ & $A P-|7-0|$ & No & $2.3 \mathrm{E}+00$ & No \\
\hline Nicked & $6 / 6$ & $8.6 \mathrm{E}+00$ & $2.5 \mathrm{E}+0 \mathrm{i}$ & $\begin{array}{l}A \overrightarrow{A P}-01-01 \\
A P-22-01\end{array}$ & $\begin{array}{l}A P-01-02 \\
A P-23=01\end{array}$ & $A P-17-01$ & N/A & $1.6 E+02$ & No \\
\hline Polassiun & $6 / 6$ & $3.5 E+02$ & $1.9 \mathrm{E}+03$ & $\begin{array}{l}A P-01-01 \\
A P-22-01\end{array}$ & $\begin{array}{l}A P-0,1-02 \\
A P-23-01\end{array}$ & $A P-I T-0 \mid$ & $N / A$ & $N / A$ & $N / A$ \\
\hline Selenitum & 66 & $6.6 \mathrm{E}+0 \mathrm{0}$ & $1.3 E+02$ & $\begin{array}{l}A P \cdot 01-01 \\
A P \cdot 22 \cdot 01\end{array}$ & $\begin{array}{l}\mathrm{AP}+01-02 \\
\mathrm{AP} \cdot 23-01\end{array}$ & $A P=17+01$ & NIA & $3.9 \mathrm{E}+01$ & Yes \\
\hline Silicont & $6 / 6$ & $1.1 \mathrm{E}+03$ & $1.8 \mathrm{E}+03$ & $\begin{array}{l}\text { AP.01न01 } \\
\text { AP-22-01 }\end{array}$ & $\begin{array}{l}\mathbf{A}-01-02 \\
A P-23-01\end{array}$ & AP.17-BI & $N / A$ & N/A & $N / A$ \\
\hline Silva & $6 / 6$ & $28 E+\infty 0$ & $6.2 \mathrm{E}+00$ & \begin{tabular}{|l|}
$A P-01-0 \mid$ \\
$A P-22-01$
\end{tabular} & $\begin{array}{l}A P-01402 \\
A P-23-0]\end{array}$ & $A P-17-01$ & $N / A$ & $3.9 E+01$ & No \\
\hline Sodiantra & 606 & $9.5 \mathrm{E}+00$ & $3,4 \mathrm{E}+01$ & $\begin{array}{l}\text { AP- } 01-01 \\
\text { AP- } 22-01\end{array}$ & $\begin{array}{l}A P-01-02 \\
A P-23-01\end{array}$ & $A P-17-01$ & $N / A$ & $\mathrm{~N} / \mathrm{A}$ & $N^{\prime} A$ \\
\hline Trellium & $1 / 6$ & $1.5 \mathrm{E}+00$ & $1.5 \mathrm{E}+00$ & AP-I7-0! & & & $N / A$ & $52 \mathrm{E}-1$ & $Y=s$ \\
\hline Yanadium & 66 & $63 \mathrm{E}+00$ & $4.4 \mathrm{E}+01$ & $\begin{array}{l}A P-01=01 \\
A P=22=01\end{array}$ & $\begin{array}{l}A P-01+07 \\
A P=23-01\end{array}$ & $\mathrm{AP}=\mathrm{]} 7 \mathrm{Al}$ & $N / A$ & 7.BE+ +00 & Y̌s \\
\hline Zine & $6 / 6$ & $1.3 E+0 \mid$ & $1.2 \mathrm{E}+02$ & $\begin{array}{l}\mathrm{AP}-01-01 \\
\mathrm{AP}-27-[\hat{1}]\end{array}$ & $\begin{array}{l}A P-01-02 \\
A P-23-01\end{array}$ & $A P-17=01$ & N/A & $2 . \overline{\mathrm{E}}+0 \mathrm{~s}$ & No \\
\hline
\end{tabular}

Dalasel from sompling tonducted in 2003 includes duplicale anafysis collecled for quality control.

Residentiol soil PRG at a rigk level of JE-06 and a hazard Index of 0.1 .

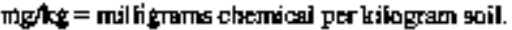

NiA - Bal avallable 


\section{Asbestes}

Asbestos was analyzed in selected sediment samples collected at ED-4. The purpose was to detemine if this substance may bave been disposed in the area due to the common use of asbestos-containing building materials during that period of time. A lotal of two (2) asbestos sarnples and one (1) duplicate sample were collected from sediment accurnulation areas identified during the walkover assessment. The sediment samples analyzed for asbestos included the 02-SD-ED4 and 04-SD-ED4, locations. A duplicate sample was collected from the 02-SD-ED4 location All sediment sample analysis results were negative for the presence of asbestos.

\subsubsection{Chemtcal Sampling Results - Soll}

\section{Data Validation Summary}

Soil samples were collected from four locations wilhin the Parcel ED-4 study area (ste Fig 6.I), with 1236 discrete andytes being validaled for this project (EPA 1999, 2004). There were 22 resuits rejected dering validation. Note that estinated values are considered acceptable information for data interpretation. The samples were collected in June 2007.

\section{Resu/ts}

The chenical sampling resuits for soil at Parcel ED-4 are summarized in Table 6.6 by analysis type and were interpreted in the risk screen (sec Appendix F). The soil data tables include the following information:

- the frequency of detection;

- minimum and maximum detected concentrations;

- location(s) at which detected:

- if detected, concentrations that exceed the clean-up levels established for Zone 1 of the ETTP;

- PRGs for the residential scenario;

- and an indication whether the PRGs were exceeded by the maximum detected concentration.

Table 6.6. Parcel ED-4 soil chemical analytical results șummiary

\begin{tabular}{lccc}
\hline \multicolumn{1}{c}{ Anatysis type } & $\begin{array}{c}\text { Frequency of } \\
\text { detecttons }\end{array}$ & $\begin{array}{c}\text { Minimum } \\
\text { concentration } \\
\text { (m-g/kg) }\end{array}$ & $\begin{array}{c}\text { Maximum } \\
\text { concentratlon } \\
\text { (mg/kg) }\end{array}$ \\
\hline METAL & $112 / 125$ & $5.3 \mathrm{E}-02$ & $5.6 \mathrm{E}+04$ \\
OTHER & $4 / 4$ & $1.0 \mathrm{E}+00$ & $1.0 \mathrm{E}+00$ \\
PPCB & $4 / 129$ & $2.0 \mathrm{E}-03$ & $5.3 \mathrm{E}-03$ \\
RADS & $332 / 332$ & $-2.6 \mathrm{E}+00$ & $5.1 \mathrm{E}+01$ \\
SVOC & $32 / 313$ & $4.0 \mathrm{E}-04$ & $2.2 \mathrm{E}-01$ \\
VOC & $4 / 240$ & $3.3 \mathrm{E}-03$ & $6.5 \mathrm{E}-0.3$ \\
\hline
\end{tabular}

Datasẹt from sampling conducted in 2007 includes duplicate amalysis collected for quality control. $\mathrm{mg} / \mathbf{k g}=$ milligrams chemical per kilogtam sol].

PPCB = pesticides and potychlorinated biphenyls.

SVOC - semivolatile organic compoumd.

VOC = volatile otgante compound. 


\subsubsection{Soil results analysis}

The following discussion summarizes the soil sampling results for ED-4.

PCRs

A total of $45 \mathrm{PCB}$ results were reported (Table 6.7), with 4 results (9\%) detected. Three of the constituents: PCB-1248 (0.0053 mg/kg), PCB-1254 (0.0036 mg/kg), and PCB-1260 (0.002 mg/kg); were detected at location NS-SSOI, while PCB-1254 was also detected at NS-SSO3. As mentioned previously, PCCBs have been used for their heat-resistant properties in oils, paints, and other materials subjected to the effects of high tempetature. The detected PCB results could be diae to the past use of oils or paints containing PCBs in the vicisity of this location, which is located between the former warehouse and the railroad spur. As presented in Table 6.7, none of the PCBs in soil had maximum detected concentrations that exceeded PRGs, indicating a low likelihood of adverse health effects from these constituents.

Table 6.7. Parcel ED-4 soi] - polychlorinated biphenyls results

\begin{tabular}{|c|c|c|c|c|c|c|c|}
\hline Analysis type & $\begin{array}{c}\text { Frequuewry } \\
\text { of } \\
\text { detections }\end{array}$ & $\begin{array}{c}\text { Minimam } \\
\text { coroceptration } \\
\text { (makg) }\end{array}$ & $\begin{array}{l}\text { Maximenm } \\
\text { concentration } \\
\text { (maskg) }\end{array}$ & $\begin{array}{l}\text { Locations } \\
\text { where } \\
\text { detected }\end{array}$ & $\begin{array}{l}\text { Exceeris } \\
\text { Zone 1 } \\
\text { remedietion } \\
\text { Ievel? }\end{array}$ & $\begin{array}{l}\text { Resident soil } \\
\text { prelinimary } \\
\text { remediation } \\
\text { (mod } \\
\text { (miky) }\end{array}$ & $\begin{array}{l}\text { Maximm m } \\
\text { detect axcesds } \\
\text { preliminary } \\
\text { remeotiatios } \\
\text { goal } \\
?\end{array}$ \\
\hline PCB-1016 & $0 / 5$ & N/A & $N / A$ & & NIA & $3.9 \mathrm{E}-0.1$ & $N / A$ \\
\hline PCB-12.21 & $0 / 5$ & $\mathrm{~N} / \mathrm{A}$ & $\mathrm{N} / \mathrm{A}$ & & $\mathrm{N} / \mathrm{A}$ & ].IE-01 & $\mathrm{N} / \mathrm{A}$ \\
\hline PCB-1232 & ars & $\mathrm{N} / \mathrm{A}$ & $\mathrm{N} / \mathrm{A}$ & & $\mathrm{N} / \mathrm{A}$ & ].IE-0I & $\mathrm{N} / \mathrm{A}$ \\
\hline$P C B-1242$ & or & $N / A$ & N/A & & N/A & 1.1E-0| & NiA \\
\hline PCB-1248 & $1 / 5$ & $5.3 \mathrm{E}-0.3$ & $5.3 \mathrm{E}-013$ & NS-SSOl-ED4 & No & I.JE-0I & No \\
\hline $\mathrm{PCB}=1254$ & $2 \sqrt{5}$ & $2,7 \mathrm{E}-03$ & $3.6 \mathrm{E}-103$ & $\begin{array}{l}\text { NS-SSOU-ED4 } \\
\text { NS-SS03-ED4 }\end{array}$ & No & $1.1 \mathrm{E}-0 \mathrm{I}$ & $\mathrm{No}$ \\
\hline $\mathrm{PCB}-1260$ & $1 / 5$ & $2.0 \mathrm{E}-03$ & $2.0 \mathrm{E}-03$ & N\$-\$SO1-ED4 & No & ],$\hat{i} \mathbf{E}=0 \mid$ & No \\
\hline $\mathrm{PCB}-1262$ & d/s & $\mathrm{N} / \mathrm{A}$ & $N A$ & & $\mathrm{~N} / \mathrm{A}$ & ]. LE-0| & $\mathbf{N} / \mathbf{A}$ \\
\hline PCCB-1268 & $0 / 5$ & $\mathrm{~N} / \mathrm{A}$ & $N / A$ & & $N / A$ & $|.1 \mathrm{E}-0|$ & $N / A$ \\
\hline
\end{tabular}

Latacet from ampling contucted in 2007 ixeludes tuplicate analysis collected for quality control.

Residential soil PRG at a risk leve] of JE-06 and a hazerd inden of 0.1

mg/kg = milligranus cherrical pa kílogram soil.

$\mathrm{N} / \mathrm{A}=\mathrm{not}$ available.

Vocs

A total of 240 VOC results were reported, with only 4 results (1.7\%) detected (Table 6.8 ). Constituents were detected at NS-SSO1 (acetone, carbot disulfide) and NS-SS02 (actene). VOCs are typically industrial solvents or components of fuel. Although the detected VOC results could be the result of releases related to former operations in this area, acetone is a common laboratory contaminant, and it is not uncommon for carbon disulfide to also be reported by laboratories in the absence of the detection of other VOCs. As presented in Table 6.8, none of the VOCs in soil had maximum detected concentrations that exceeded PRGs, indicating a low tikelihood of adverse health effects from these constituents. 
Tabie 6.8. Parcel ED-4 soilh - volatile organic compounds results

\begin{tabular}{|c|c|c|c|c|c|c|c|}
\hline Anulyss type & $\begin{array}{c}\text { Frequercy } \\
\text { of } \\
\text { detectlons }\end{array}$ & $\begin{array}{c}\text { Minlmum } \\
\text { concentration } \\
\text { (me/kf) }\end{array}$ & $\begin{array}{l}\text { Maxinum } \\
\text { concemtration } \\
\text { (mples) }\end{array}$ & $\begin{array}{l}\text { Losetions } \\
\text { whett } \\
\text { delected }\end{array}$ & $\begin{array}{c}\text { Ercetds } \\
\text { Zane } 1 \\
\text { reanediatien } \\
\text { Level? }\end{array}$ & $\begin{array}{c}\text { Resident soil } \\
\text { prelimimary } \\
\text { remedinlios } \\
\text { poll } \\
\text { [molkt] }\end{array}$ & 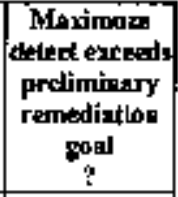 \\
\hline 1,1,I-Trichlorosthine & d/s & N/A & $\mathbf{N} / \mathbf{A}$ & & N/A & $2,0 \mathrm{E}+02$ & $\mathbf{N} / \mathbf{A}$ \\
\hline $1,2,2, T$ trachloroethant & OWS & N/A & N/A & & $\mathrm{N} / \mathrm{A}$ & 4.1E-[0] & $N A$ \\
\hline $\begin{array}{l}\text { 1,1,2-Trichkoro-1,2,2- } \\
\text { nifhequedhant }\end{array}$ & $0 / 5$ & $N / A$ & $\mathrm{~N} / \mathrm{A}$ & & $\dot{\mathrm{N}} / \dot{A}$ & $2.1 \mathrm{E}+03$ & N/A \\
\hline 1,1,2-Trichlorodhoure & QN5 & NA & $\mathrm{N} / \mathrm{A}$ & & N/A & $7.3 \mathrm{E}-0 \mathrm{I}$ & $N / A$ \\
\hline I,I-Dichloroethatle & ON' & $\mathrm{NA}$ & NA & & $\mathbf{N} / \mathbf{A}$ & $5.1 E+0]$ & $\mathrm{N} / \mathrm{A}$ \\
\hline I, I-Dixhlorothene & 0 or 5 & NA & $N / A$ & & $\mathrm{~N} / \mathrm{A}$ & $12 E+01$ & $N / A$ \\
\hline $1,2,3$-Trichtorobermzene & $0 / 5$ & $N / A$ & N/A & & $\mathbf{N} / \mathbf{A}$ & $\mathrm{N} / \mathrm{A}$ & $\mathrm{N} / \mathrm{A}$ \\
\hline $\begin{array}{l}\text { 1,2-Dibronk-3- } \\
\text { clilgropropente }\end{array}$ & $0 / 5$ & $\bar{N} / \mathrm{A}$ & $\mathrm{N} / \mathrm{A}$ & & N/A & 2.IE-01 & NIA \\
\hline 12-Dibroknoethane & o/s & $\mathrm{N} / \mathrm{A}$ & $N / A$ & & $\mathrm{~N} / \mathrm{A}$ & $3.2 \mathrm{E}-02$ & N/A \\
\hline 1,2-Dichlorothame & ofs & $\mathrm{N} / \mathrm{A}$ & $\mathrm{NA}$ & & $N / A$ & $2.8 \mathrm{E} \cdot 0 \mathrm{01}$ & $\overline{N A}$ \\
\hline 1,2-Dichloraporianse & N/5 & NA & NA & & $N A$ & $3.4 E-0]$ & $\mathrm{N} / \mathrm{A}$ \\
\hline 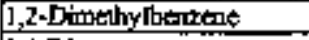 & $0 / 5$ & $N / A$ & N/A & & $N A$ & $2.7 \mathrm{E}+0 \mathrm{I}$ & N/A \\
\hline 1,4-Dioxante & ous & $\mathrm{NA}$ & $N_{A}$ & & N/A & 4.4E+Q1 & $N / A$ \\
\hline 2-Butanone & $0 \sqrt{5}$ & $\mathrm{NA}$ & $\mathrm{N} / \mathrm{A}$ & & $N / A$ & $22 E+03$ & N/A \\
\hline 2-Hexancone & $0 / 5$ & NA & $\mathrm{N} / \mathrm{A}$ & & $\mathrm{N} / \mathrm{A}$ & $N / A$ & $\mathrm{NAA}$ \\
\hline $\begin{array}{l}\text { 2-Methory-2- } \\
\text { pisethylpropane }\end{array}$ & ors & $\overline{N / A}$ & $W_{A}$ & & $\mathrm{~N} / \mathrm{A}$ & 1.JE+01 & $\mathrm{N} / \mathrm{A}$ \\
\hline 4-vetedy-2-penlamone & $0 / 5$ & $\mathrm{~N} / \mathrm{A}$ & $\mathrm{NA}$ & & $\mathrm{N} / \mathrm{A}$ & $5.3 E+02$ & $\mathrm{~N} / \mathrm{A}$ \\
\hline Acetone & $3 / 5$ & $3.3 \mathrm{E}-0.3$ & $5.0 \mathrm{E}-03$ & $\begin{array}{l}\text { NS-SSO]-ED4 } \\
\text { NS-SSO2-EDA }\end{array}$ & $N / A$ & $1,4 E+03$ & No \\
\hline Brizeale & Qi5 & $N A$ & $\mathrm{NA}$ & & $\mathrm{N} / \mathrm{A}$ & $6.4 E-0]$ & $\mathrm{N} / \mathrm{A}$ \\
\hline Bromechloromethune & $0 / 5$ & $\mathrm{~N} / \mathrm{A}$ & $N A$. & & $\mathrm{N} / \mathrm{A}$ & $\mathrm{N} / \mathrm{A}$ & $N / A$ \\
\hline Bromodichlonomethane & $0 / 3$ & NA & $\mathrm{N} / \mathrm{A}$ & & $N / A$ & $82 \mathrm{E}-1$ & $\mathrm{~N} / \mathrm{A}$ \\
\hline Bmengform & $0 / 5$ & $\mathbf{N A}$ & $N / A$ & & $\mathrm{~N} / \mathrm{A}$ & $6.2 E+0]$ & $\mathrm{N} / \mathrm{A}$ \\
\hline Bromometrane & $0 / 5$ & N/A & $N_{A}$ & & $\mathrm{~N} / \mathrm{A}$ & $3.9 \mathrm{E}-\mathrm{I}$ & $\mathrm{N} / \mathrm{A}$ \\
\hline Carbong disulfipde & $1 / 5$. & $6.5 \mathrm{E}-03$ & $6.5 \overline{\mathrm{E}} \overline{0.35}$ & NS-SSOJ-EDA & $N / A$ & $3.6 \mathrm{E}+01$ & $\mathbf{N o}$ \\
\hline Carbon tetrechlonide & $0 / 5$ & $\mathrm{~N} / \mathrm{A}$ & N/A & & $N / A$ & $2.2 \mathrm{E}-01$ & $\overline{\mathrm{N}} / \mathrm{A}$ \\
\hline Chlorobenzente & $0 / 5$ & $\mathrm{~N} / \mathrm{A}$ & $\mathrm{NA}$ & & $N / A$ & $1.5 \mathrm{E}+01$ & $N / A$ \\
\hline Chloronthane & $0 / 5$ & $N / A$ & $\mathrm{~N} / \mathrm{A}$ & & $\mathrm{N} / \mathrm{A}$ & $3,0 \mathrm{E}+00$ & N/A \\
\hline Charoform & $0 \sqrt{5}$ & $\mathrm{~N} / \mathrm{A}$ & $\mathrm{NA}$ & & $\mathrm{N} / \mathrm{A}$ & $2.2 \mathrm{E}-0 \mathrm{l}$ & $\bar{W} / A$ \\
\hline thloromethank & $0 / 5$ & $N \tilde{N}$ & N/A & & $\mathrm{N} / \mathrm{h}$ & 4.TE+0.0 & $\mathbf{N} / \mathbf{A}$ \\
\hline Cumbene & $0 / 5$ & $\mathrm{~N} / \mathrm{A}$ & N/A & & $N / A$ & {$[.6 \mathrm{E}+0]$} & $\sqrt{/ A}$ \\
\hline Cyclohexane & $0 / 3$ & $N / A$ & N/A & & N/A & $1,4 E+02$ & $N / A$ \\
\hline Dithropophlaromethanc & 0,5 & $\mathrm{~N} / \mathrm{A}$ & $N / A$ & & $\mathrm{~N} / \mathrm{A}$ & $1.1 \mathrm{E}+00$ & $\mathrm{~N} / \mathrm{A}$ \\
\hline Diehlarodíflowomethant & $0 / 5$ & N/A & $\mathrm{N} / \mathrm{A}$ & & $N / A$ & $9.4 \mathrm{E}+6 \mathrm{~B}$ & $\mathrm{~N} / \mathrm{A}$ \\
\hline Ellyylberzene & $0 / 5$ & $N / A$ & $\mathrm{~N} / \mathrm{A}$ & & $\mathrm{N} / \mathrm{A}$ & $1.9 \mathrm{E}+02$ & $N / A$ \\
\hline$M+P X y l a n t$ & $0 \sqrt{5}$ & $\mathrm{~N} / \mathrm{A}$ & $N / A$ & & $N / h$ & $2.7 \mathbf{E}+01$ & NA \\
\hline Mettyl acetate & ors & $N / A$ & $N / A$ & & $N A$ & $2.2 \mathrm{E}+63$ & $\mathrm{~N} / \mathrm{A}$ \\
\hline Methylsycichexsane & $0 / 5$ & $N / A$ & $\mathrm{~N} / \mathrm{A}$ & & $\mathrm{N} / \mathrm{A}$ & $2.6 \mathrm{E}+02$ & $N / A$ \\
\hline Methylega chillopide & $0 / 5$ & $N / A$ & NA & & N/A & 9.IE+00 & $N / A$ \\
\hline Sxyted & Dis & $N / A$ & $\mathbf{N} / \mathrm{A}$ & & $\mathbf{N A}$ & $4.4 E+02$ & $N / A$ \\
\hline Tetrachloroetianse & $0 \sqrt{5}$ & $\mathrm{~N} / \mathrm{A}$ & $N / A$ & & NA & 4.85-01. & $\mathrm{N} / \mathrm{A}$ \\
\hline Tolucare & 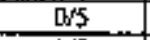 & $N_{A}$ & $N / A$ & & $N / A$ & $6.6 \mathrm{E}+01$ & $N / A$ \\
\hline Trishlondelhene & $0 / 5$ & $\mathrm{~N} / \mathrm{A}$ & $\mathrm{N} / \mathrm{A}$ & & $\mathrm{N} / \mathrm{A}$ & $5.3 E-102$ & N/A \\
\hline Trichloroflikongenthane & $0 / 3$ & $\sqrt{N / A}$ & $\mathrm{~N} / \mathrm{A}$ & & $\mathrm{N} f \mathrm{~A}$ & $3.9 \mathrm{E}+(0 \mathrm{]}$ & $\mathrm{N} / \mathrm{A}$ \\
\hline Vinylchloride & $a / 5$ & N/A & $\mathrm{N} / \mathrm{A}$ & & NiA & $7.9 E-02$ & $\mathrm{~N} / \mathrm{A}$ \\
\hline cits-1,2-Dichlotokthile & $0 / 5$ & $\mathrm{~N} / \mathrm{A}$ & $N / A$ & & $\mathrm{~N} / \mathrm{A}$ & $4.3 E+00$ & N/A \\
\hline ers-1,3-Djchloropepene & 0,5 & $N / A$ & $N / A$ & & $N / A$ & $7.8 \mathrm{E}-101$ & $\sqrt{1 / A}$ \\
\hline frams-1,2-Dichlonothene & 2015 & NA & N/A & & NA & $6.9 \mathrm{E}+00$ & $\mathrm{~N} / \mathrm{A}$ \\
\hline Erans-1, 3-Dichloripprepene & $0 / 5$ & N/A & $N / A$ & & $\sqrt[N]{ } / \mathrm{A}$ & 7.8E-0! & $\mathrm{N} / \mathrm{A}$ \\
\hline
\end{tabular}

Dulasel fron sampling booducted in 2007 inchrdes tupilicate asalysis colleciled for quality contol. Residential soil PRG al a jigk leved of IE-06 and a hazard index of 0.1 .

$\mathbf{m g} / \mathbf{k g}=$ milligrams chemical per kilogtalis sot.

NAA = mot applicabie. 


\section{SVOCs}

A total of 313 SVOC results were reported (Table 6.9), with 32 results (10\%) detected. SVOCs ase used in various industries and found in lubricants and as components of fuel. Detections ranged from $0.0004 \mathrm{mg} / \mathrm{kg}$ for 1,4-dichlorobenzene at NS-SSO3 to $0.222 \mathrm{mg} / \mathrm{kg}$ for benzo(a)anthracene at NS-SS01, with detections at all locations. The detected SVOC results could be the result of runoff from adjoining asphalt artas or the use of diesel fuel andor lubricating oils associated with activities along the former railroad. As indicated in Table 6.9 , benzo( $a$ )pyrene had a maximum detected soil concentration in excess of the residential soil PRG. The potential for adverse health effects associated with concentrations excetding the $\mathrm{PRG}$ is discussed in the risk evaluation (Appendix F).

Table 6.9. Parcel ED-4 soi- semirvolatile organic compounds Iesults

\begin{tabular}{|c|c|c|c|c|c|c|c|}
\hline L. Antolysis type & $\begin{array}{c}\text { Frequency } \\
\text { of } \\
\text { detections }\end{array}$ & $\begin{array}{c}\text { M0nImum } \\
\text { concentratian } \\
\text { (mig'ks) }\end{array}$ & 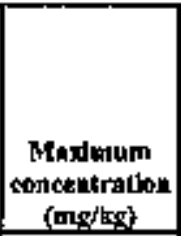 & $\begin{array}{c}\text { Locationt where } \\
\text { detected }\end{array}$ & $\begin{array}{c}\text { Exceeds } \\
\text { Zonte 1 } \\
\text { renetiation } \\
\text { levelf } \\
\end{array}$ & $\begin{array}{c}\text { Residem } \\
\text { noil } \\
\text { perliminary } \\
\text { remediatlon } \\
\text { soal } \\
\text { (moiks) }\end{array}$ & $\begin{array}{c}\text { Maximum } \\
\text { delee: } \\
\text { exceads } \\
\text { prellminary } \\
\text { remediation } \\
\text { onal } \\
?\end{array}$ \\
\hline 1,2,4,5-Teriacblorobentente & 015 & $\mathrm{~N} / \mathrm{A}$ & NiA & & N/A & $1.8 \mathrm{E}+0 \mathrm{OW}$ & $\mathrm{N} / \mathrm{A}$ \\
\hline 1,2,4-Trichlonobrazerik & $0 / 5$ & $\mathrm{~N} / \mathrm{A}$ & $N / A$ & & $N / A$ & $6.2 \mathrm{E}+00$ & N/A \\
\hline 1,2-Dichlorobenzene & 015 & N/A & $\mathrm{N} / \mathrm{A} / \mathrm{H}$ & & N/A & l.IE 402 & $\overline{N / A}$ \\
\hline 1,3 -Dichloraberizent & $0 / 5$ & $\mathrm{~N} / \mathrm{A}$ & $N / A$ & & $\overline{N / A}$ & $5.3 \mathrm{E}+0 \mathrm{I}$ & $\mathrm{N} / \mathrm{A}$ \\
\hline 1,4-Dichlorobenzene & $2 / 5$ & $\$, 0 \mathrm{E}-144$ & $6.5 \mathrm{E}-04$ & NS-SSO2-ED4 NS-SSO3-ED4 & $\mathrm{N} / \mathrm{A}$ & $3.4 \mathrm{E}+00$ & $N_{0}$ \\
\hline 2,3,4,6-Tetrachlorepheral & $0 / 5$ & $\mathrm{~N} / \mathrm{A}$ & $\mathrm{N} / \mathrm{A}$ & & $\mathbf{N} / \mathrm{A}$ & $1.8 \mathrm{E}+02$ & N/A \\
\hline 2,4,5-Trichiorophenol & $0 / 5$ & $\mathbf{N A}$ & $\mathbf{N} / \mathrm{A}$ & & $\mathrm{N/A}$ & $6.1 E+02$ & $N / A$ \\
\hline 2,4,6-Trichlotophenol & $2 / 5$ & $\mathrm{~N} / \mathrm{A}$ & $\mathrm{N} / \mathrm{A}$ & & $N / A$ & $0.1 \mathrm{E}-01$ & $N / A$ \\
\hline 2,4-Dithlorophenol & $0 / 5$ & $\mathrm{~N} / \mathrm{A}$ & $\mathrm{N} / \mathrm{A}$ & & $\mathbf{N} / \mathrm{A}$ & $1.8 \mathrm{E}+0 \mid$ & $\mathrm{N} / \mathrm{A}$ \\
\hline 2,4-Dimethylphenol & $0 / 5$ & $\mathrm{~N} / \mathrm{A}$ & $N / A$ & & $N A$ & $12 \mathrm{E}+02$ & $N / A$ \\
\hline 2,4-Diritrophenol & $0 / 5$ & $\mathrm{~N} / \mathrm{A}$ & N/A & & $N / A$ & I2E+0I & N/A \\
\hline 2,d-Dimitrotolustines & as & N/A & $\mathrm{N} / \mathrm{A}$ & & $\mathrm{N} / \mathrm{A}$ & $7.2 \mathrm{E}-01$ & $\mathrm{NA}$ \\
\hline 2,6-Diuitrofoluene & 045 & $\mathrm{~N} / \mathrm{A}$ & $\mathrm{N} / \mathrm{A}$ & & $\mathrm{N} / \mathrm{A}$ & $7.2 \mathrm{E}-0 \mathrm{I}$ & $\mathrm{N} / \mathrm{A}$ \\
\hline 2-Chlorothaphthalene & $0 / 5$ & $\mathrm{~N} / \mathrm{A}$ & $\mathrm{N} / \mathrm{A}$ & & $\mathrm{N} / \mathrm{A}$ & $4.9 E+02$ & $N / A$ \\
\hline 2-Chbiknophend & $0 / 5$ & $N / A$ & $\mathrm{~N} / \mathrm{A}$ & & $\mathrm{N} / \mathrm{A}$ & $6.3 \mathrm{E}+00$ & $\mathrm{~N} / \mathrm{A}$ \\
\hline 2-Methyl-4,6-dinitrophenol & ars & $\mathrm{NA}$ & $N / A$ & & $\overline{N / A}$ & $6.1 \mathrm{E}-01$ & $\mathrm{NA}$ \\
\hline 2-twethylthaphthalene & $0 / 5$ & $N / A$ & N/A & & $N^{\prime} \mathrm{A}$ & $5.6 \mathrm{E}+00$ & NA \\
\hline 2-Methylphanol & ois & $\mathbf{N} / \mathbf{A}$ & $\mathrm{N} / \mathrm{A}$ & & $N / A$ & $3.1 \mathrm{IE}+02$ & $\mathrm{~N} / \mathrm{A}$ \\
\hline 2-Nitrobeturtakenine & ars & N/A & $N_{A}$ & & $\mathrm{NA}$ & $1.8 \mathrm{E}+0 \mathrm{I}$ & $\mathrm{N} / \mathrm{A}$ \\
\hline 2-Nirrophengl & D/5 & $\mathbf{N} / \mathbf{A}$ & $\mathrm{N} / \mathrm{A}$ & & $\mathrm{N} / \mathrm{A}$ & $\mathbf{N} / \mathbf{A}$ & $\mathrm{N} / \mathrm{A}$ \\
\hline 3-Nitrobenzeramine & $0+5$ & $\mathbf{N} / \mathbf{A}$ & $N / A$ & & $N / A$ & I.GE+OD & $\overline{N_{A}}$ \\
\hline 6-Bromophenyl phard the & as & $\mathrm{N} / \mathrm{A}$ & $\bar{N} / \mathrm{A}$ & & $\mathrm{N} / \mathrm{A}$ & $\mathrm{N} / \mathrm{A}$ & $N / A$ \\
\hline 4-ohora-3-methylphenol & 0,5 & $\mathbf{N} / \mathrm{A}$ & $\mathrm{N} / \mathrm{A}$ & & $\mathrm{N} / \mathrm{A}$ & $\mathrm{N} / \mathrm{A}$ & $\mathrm{N} / \mathrm{A}$ \\
\hline 4-Chloroberizentamine & ass & N/A & NA & & $\mathrm{N} / \mathrm{A}$ & $2.4 \mathrm{E}+01$ & NA \\
\hline 4-Chloropheny1 pherry 1 epher & Dots & $\mathbf{N} / \mathrm{A}$ & $\mathrm{N} / \mathrm{A}$ & & $\mathrm{N} / \mathrm{A}$ & $\mathrm{N} / \mathrm{A}$ & $\mathrm{N} / \mathrm{A}$ \\
\hline 4-Nitrobtemenamine & $0 / 5$ & $\mathrm{~N} / \mathrm{A}$ & $\mathrm{N} / \mathrm{A}$ & & $\mathrm{N} / \mathrm{A}$ & $1.8 \mathrm{E}+01$ & $\mathbf{N} / \mathrm{A}$ \\
\hline 4-Nitrophengl & $0 / 3$ & $\mathrm{~N} / \mathrm{A}$ & $\mathrm{N} / \mathrm{h}$ & & $\mathrm{N} / \mathrm{A}$ & $\mathrm{N} / \mathrm{A}$ & $N / A$ \\
\hline Acensphthent & 015 & $\mathrm{~N} / \mathrm{A}$ & $\mathrm{NA}$ & & NIA & $3.7 \mathrm{E}+02$ & $N / A$ \\
\hline Acenaphthylere & $0 / 5$ & $\mathrm{~N} / \mathrm{A}$ & $N / A$ & & $\mathrm{~N} / \mathrm{A}$ & $3.7 \mathrm{E}+02$ & $N / A$ \\
\hline Acetophethone & ars & $N / A$ & $\mathrm{~N} / \mathrm{A}$ & & $\mathrm{N} / \mathrm{A}$ & $\mathrm{N} / \mathrm{A}$ & $\mathrm{N} / \mathrm{A}$ \\
\hline Anulluatcenr & $0 / 5$ & $N / A$ & $\mathrm{NA}$ & & $\mathrm{N} / \mathrm{A}$ & $2.2 \mathrm{E}+03$ & $\mathrm{~N} / \mathrm{h}$ \\
\hline Benz(a)anthracene & $2 / 5$ & $3.0 \mathrm{E}-02$ & $3.6 \mathrm{E}-02$ & NS-SSÓl-ED4 & NAS & $6.2 \mathrm{E}-01$ & $N o$ \\
\hline Bedzo(a)pytene & $4 \sqrt{4}$ & {$[.6 \mathrm{E}-0]$} & l.7E-0I & $\begin{array}{l}\text { NS-SS01-ED4 N5-5S03-ED4 } \\
\text { NS-SS04-ED4 }\end{array}$ & $N / A$ & $6.2 \mathrm{E}-12$ & Yes \\
\hline
\end{tabular}


Table 6.9. Parcel ED-4 soil - senivodatile orgenic compounds results (continued)

\begin{tabular}{|c|c|c|c|c|c|c|c|}
\hline Analy회 type & $\begin{array}{c}\text { Prequenky } \\
\text { of } \\
\text { detections }\end{array}$ & $\begin{array}{c}\text { Minimum } \\
\text { concentratlon } \\
\text { (mox/kg) }\end{array}$ & $\begin{array}{c}\text { Muximum } \\
\text { cancentration } \\
\text { (mog/kg) }\end{array}$ & $\begin{array}{c}\text { Lacatinas where } \\
\text { detected }\end{array}$ & $\begin{array}{c}\text { Exceets } \\
\text { Zone I } \\
\text { zemediation } \\
\text { level? }\end{array}$ & 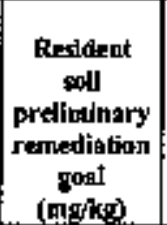 & $\begin{array}{l}\text { Maximum } \\
\text { delect } \\
\text { eretedt } \\
\text { prelietinary } \\
\text { remetifation } \\
\text { goal } \\
\text { ? }\end{array}$ \\
\hline 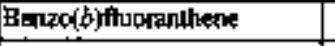 & $2 / 4$ & $2 . I E-0 \mid$ & $22 \mathrm{E}-0 \mathrm{I}$ & NS-S\$ON-ED4 N5-S\$S03-ED4 & $\mathrm{N}_{\mathrm{A}}$ & $6.2 \mathrm{E}-01$ & No \\
\hline Bertzo(g,h, iperyleate & $3 / 3$ & $6.9 \mathrm{E}-02$ & $8.0 \mathrm{E}-02$ & NS-SSBI-ED4 NS-SSO4-ED4 & N/A & $2.3 \overrightarrow{\mathrm{E}}+02$ & $\mathrm{No}$ \\
\hline Benow(k) fifurranthene & $1 / 3$ & $32 \mathrm{E}=03$ & $32 \mathrm{E}-02$ & NS-SSAl-ED4 & N/A & $6.2 \mathrm{E}+00$ & No \\
\hline Bis(2-chlordethoxy)methane & $0 / 5$ & $\mathrm{~N} / \mathrm{A}$ & $\mathrm{NA}$ & & N/A & NA & N/A \\
\hline Bis (2-ebikoethyl) ether & $0 / 5$ & N/A & N/A & & $N / A$ & $2.2 \mathrm{E}-01$ & NA \\
\hline Bis(2-thylhexyl )phtbalate & $0 / 5$ & $\mathrm{NA}$ & NA & & $\mathrm{N} / \mathrm{A}$ & $3.5 \mathrm{E}+0 \mathrm{I}$ & $\mathrm{N} / \mathrm{A}$ \\
\hline Bury] benzyd philhalate & ors & N/A & $N / A$ & & N/A & $1.2 \mathrm{E}+03$ & N/A \\
\hline Crubazole & 045 & $\mathrm{~N} / \mathrm{A}$ & $\mathrm{N} / \mathrm{A}$ & & $N A$ & $2.4 \mathrm{E}+01$ & $\mathrm{~N} / \mathrm{A}$ \\
\hline Chrysene & 45 & $1.8 \mathrm{E}-02$ & $3.9 \mathrm{E}-02$ & $\begin{array}{l}\text { NSS-SS4I-ED4 NS-5S03-ED4 } \\
\text { NS-SSB4-ED4 }\end{array}$ & NA & $6.2 \mathbf{E}+41$ & $\mathrm{No}_{\mathrm{p}}$ \\
\hline Di-n-butyd phalbabie & $0 / 5$ & $N / A$ & $\mathrm{~N} / \mathrm{A}$ & & $N / A$ & $6.1 E+02$ & $N / A$ \\
\hline Di-n-DCtylphththalate & $0 / 3$ & $N / A$ & $N / A$ & & N/A & $2.4 \mathrm{E}+02$ & $\mathrm{~N} / \mathrm{A}$ \\
\hline Dibenz(a,h)anthracene & $0 / 3$ & $N / A$ & $N / A$ & & W/A & $62 \mathrm{E}-02$ & $N / A$ \\
\hline Dibreofinath & $\alpha / 5$ & $\overline{N / A}$ & $N / A$ & & $\mathrm{~N} / \mathrm{A}$ & $1.5 E+[0]$ & $\mathrm{N} / \mathrm{A}$ \\
\hline Diethyl phifhalate & $6 / 5$ & $\mathbf{N} / \mathbf{A}$ & $\mathrm{N} / \mathrm{A}$ & & $\mathrm{N} / \mathrm{A}$ & $4.9 \mathrm{E}+03$ & N/A \\
\hline Dimethy] phthable & $0 / 5$ & $N / A$ & $N / A$ & & NA & $6 . \overline{\mathrm{E}+04}$ & N/A \\
\hline Diphtenylimine & $0 / 5$ & $N / A$ & $N / A$ & & N/A & $1.5 E+02$ & $\mathbf{N} / A$ \\
\hline F] woradthent & 45 & $2.5 \mathrm{E}-02$ & $5.7 \mathrm{E}-102$ & $\begin{array}{l}\text { NS-SSOI-ED4 NS-SS03-ED4 } \\
\text { NS-SSO4-ED4 }\end{array}$ & NA & $2.3 \mathrm{E}+02$ & No \\
\hline Fluotent: & $0 \sqrt{5}$ & $\mathrm{~N} / \mathrm{A}$ & $\mathrm{N} / \mathbf{A}$ & & W/A & $2.7 \mathrm{E}+\mathrm{Q2} 2$ & $\mathrm{~N} / \mathrm{A}$ \\
\hline Herachiortbaniene" & ors & $N / A$ & NA & & $\mathrm{N} / \mathrm{A}$ & $3.0 \mathrm{E}-10 \mathrm{I}$ & $N / A$ \\
\hline Mexachlorptrotadiene & ois & $\mathrm{N} / \mathrm{A}$ & $N A$ & & $N / A$ & $1.8 E+00$ & $\bar{N} / \mathrm{A}$ \\
\hline Hexachlorocyclopets tadiene & ows & $\mathrm{N} / \mathrm{h}$ & $\mathrm{N} / \mathrm{A}$ & & $\mathrm{N} / \mathrm{A}$ & $3.7 \mathrm{E}+\mathrm{OI}$ & N/A \\
\hline Fexachlortethane & $0 / 5$ & $\mathrm{~N} / \mathrm{A}$ & $\mathrm{N} / \mathrm{A}$ & & $\mathrm{N} / \mathrm{A}$ & $6.15+00$ & $\mathrm{~N} / \mathrm{A}$ \\
\hline Indeno $(1,2,3,-\operatorname{cod})$ pytente & $3 \sqrt{3}$ & $1.6 \mathrm{E}-02$ & $2,3 \mathrm{E}-122$ & NS-SSGI-EDA NS-SSA4-ED4 & $\mathrm{N} / \mathrm{A}$ & $6.2 \mathbf{E}-01$ & No \\
\hline Lophorone & $0+5$ & $\mathrm{~N} / \mathrm{A}$ & $\mathrm{N} / \mathrm{A}$ & & N/A & $5.1 \mathrm{E}+02$ & $\mathrm{~N} / \mathrm{A}$ \\
\hline N-Nilroso-di-n-propylantine & 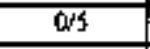 & $N^{\prime} \mathrm{A}$ & $N / A$ & & N/A & $6.9 E-02$ & $\mathrm{~N} / \mathrm{A}$ \\
\hline Naphthalche & ors & $N / A$ & $\mathrm{~N} / \mathrm{A}$ & & $N / A$ & $5.6 \mathrm{E}+00$ & $\mathrm{~N} / \mathrm{A}$ \\
\hline Nitubenzene & avs & $\mathrm{N} / \mathrm{A}$ & $\mathrm{N} / \mathrm{A}$ & & N/A & $2.0 \mathrm{E}+40$ & $\mathrm{~N} / \mathrm{A}$ \\
\hline Pentachlorophenoil & $6 / 5$ & $\mathrm{NA}$ & $W / A$ & & $\mathrm{~N} / \mathrm{A}$ & $3.6 \mathrm{E}+00$ & $\mathrm{~N} / \mathrm{A}$ \\
\hline Ficjanthyent & $2 / 3$ & $1.2 E-42$ & $3,0 \mathrm{E}-02$ & NS-SSO1-ED4 NS-SSO4EDA & $\mathrm{N} / \mathrm{h}$ & $2.3 \mathrm{E}+02$ & No \\
\hline Phenod & $0 A S$ & N/A & $\mathrm{N} / \mathrm{A}$ & & $W A$ & $1.8 \mathrm{E}+03$ & $\mathrm{~N} / \mathrm{A}$ \\
\hline Pyrene & $5 / 5$ & $1.9 \mathrm{E}-02$ & $9.0 \mathrm{E}-102$ & $\begin{array}{l}\text { NS-SSOI-ED4 NS-SSO2-ED4 } \\
\text { NS-SS63-ED4 NS-SS44-ED4 }\end{array}$ & N/A & $2.3 E+02$ & $\mathrm{No}$ \\
\hline Hip Methy]phenol & $0 / 5$ & $\mathrm{~N} / \mathrm{A}$ & $\mathrm{NA}$ & & $\mathrm{N} / \mathrm{A}$ & $\mathbf{N} / A$ & $\mathrm{~N} / \mathrm{A}$ \\
\hline
\end{tabular}

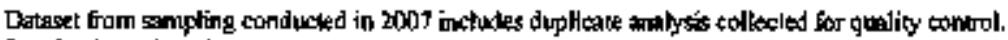

Recidential soil PRG at a rigk ked of ]E-06 and a bezerd index orO.1.

$\mathrm{mg} / \mathrm{kg}$ - melligrams themicsl per kikeram soil.

N/A = not applicable

\section{Pestictides}

A total of 84 pesticide results were reported, with no detections.

\section{Herbicides}

A total of 24 herbicide results were reported, with no detections. 


\section{Metals}

A total of 125 metais results were reported, with 112 results $(90 \%)$ detected. The detected concentrations ranged from $0.09 \mathrm{mg} / \mathrm{kg}$ for mercury at NS-SSO2 to $56,000 \mathrm{mg} / \mathrm{kg}$ for chlcium at NS-\$S0201 (Table 6.10). All sample locations were found to have detected metals results, which is to be expected because soil generally has a measurable content of metals in nature. There is no evident correlation benveen sample location and elevated metals results. The highest metals results were for the macronutrient elements calcium, iron, manganese, magnesium, alumirum, and potassium, which are all naturally occurring. As indicated in Table 6.10, several metals had a maximum detected soil concentration in excess of the residential soil PRG. However, the established ETTP background soil concentrations for gix of these seven metals are higher than the PRG, and only the maximum coneentration of selenim exceted both the PRG and background concentrations. The potential for adverse health effects associated with concentrations exceeding the PRG is discussed in the risk evaluation (Appendix F).

Tsble 6.10. Parcel ED-4 study area soil - metals results

\begin{tabular}{|c|c|c|c|c|c|c|c|}
\hline Anslysts type & $\begin{array}{c}\text { Frequency } \\
\text { of } \\
\text { defections }\end{array}$ & 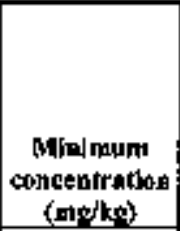 & $\begin{array}{c}\text { Maxlmum } \\
\text { collceatralion } \\
\text { (mijkg) }\end{array}$ & $\begin{array}{c}\begin{array}{c}\text { Lorations where } \\
\text { detected }\end{array} \\
\end{array}$ & $\begin{array}{c}\text { Excteds } \\
\text { Zone 1 } \\
\text { remeqtiation } \\
\text { level? }\end{array}$ & $\begin{array}{c}\text { Regbdent } \\
\text { gol] } \\
\text { prelimilasry } \\
\text { remediatton } \\
\text { goal } \\
\text { (mig/kg). }\end{array}$ & 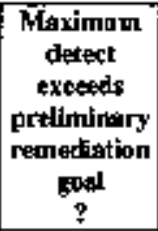 \\
\hline Aluminum & $5 / 5$ & $8.1 \mathbf{E}+03$ & $1.2 \mathrm{E}+04$ & $\begin{array}{l}\text { NS-SSOOI-EDA NS-SSO2-ED4 } \\
\text { NS-SSO3-ED4 NS-SSO4-ED4 }\end{array}$ & N/A & $7,6 \mathrm{E}+0.3$ & Yes \\
\hline Antimony & $2 / 5$. & $1.3 \mathrm{E}+00$ & $1.5 \mathrm{E}+00$ & NS-SSOJ-ED4 NS-SS04-ED4 & $\mathrm{N} / \mathrm{A}$ & $3,1 \mathbf{E}+00$ & No \\
\hline Arsenic & $\$ / 5$ & $3,0 E+00$ & $6.5 E+00$ & $\begin{array}{l}\text { NSSSSOI-EDA NS-SSTO2-ED4 } \\
\text { NST-SSO3-ED4 NS-SSO4-ED4 }\end{array}$ & No & $3.9 \mathrm{E}-0 \mathrm{I}$ & Yes \\
\hline Barimm & $5 / 5$ & $3,4 \mathrm{E}+0 \mathrm{I}$ & $2.9 \mathrm{E}+0 \mathbf{2}$ & 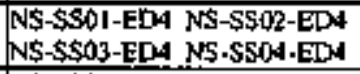 & $N / A$ & $5,4 \mathrm{E}+02$ & No \\
\hline Beтryllinm & $s / s$ & $2.9 \mathrm{E}-01$ & $12 \mathrm{E}+00$ & $\begin{array}{l}\text { NS-SSO1-EDA NS-SS02-ED4 } \\
\text { NS-SS03-ED4 NS-SS04-ED4 }\end{array}$ & No & $1.5 \mathrm{E}+0 \mathrm{I}$ & No \\
\hline Eoron & $\overline{5 / 3}$ & $1.4 \mathrm{E}+\mathrm{CH}$ & $7.5 E+00$ & $\begin{array}{l}\text { NS-SS01-ED4 NS-SS02-EDA } \\
\text { NS-SS03-ED4 NS-SSO4-ED4 }\end{array}$ & $\mathrm{N} / \mathrm{A}$ & $9.6 \mathrm{E}+03$ & No \\
\hline Caktmium & $2 / 5$ & I.6E-Dl & $2.4 \mathrm{E}-0 \mathrm{I}$ & NS-S\$02-ED4 NS-SSOL4-EDA & $\mathrm{N} / \mathrm{A}$ & 3.7 400 & No \\
\hline Calcium & $5 / 3$ & $2.9 E+03$ & $5.6 \mathrm{E}+04$ & $\begin{array}{l}\text { NS-SSO1-ED4 NS-S502-ED4 } \\
\text { NS-SS03-ED4 NS-SSO4-ED4 }\end{array}$ & $N / A$ & $\mathrm{~N} / \mathrm{A}$ & $N / A$ \\
\hline Chrottintm & $5 / 5$ & $1.2 \mathrm{E}+0 \mathrm{l}$ & $3.1 \mathrm{E}+01$ & $\begin{array}{l}\text { NS-SS01-ED4 NS-SSO2-ED4 } \\
\text { NS-SS03-ED4 NS-SSO4-EL4 }\end{array}$ & $\mathrm{NA}$ & $22 \mathrm{E}+0 \mathrm{~d}$ & $Y=3$ \\
\hline Cobali & $5 / 5$ & $4.0 \mathrm{E}+00$ & ].6E+01 & $\begin{array}{l}\text { NS-S501-ED4 NS-SSO2-ED4 } \\
\text { NS-SSSOJ-ED4 NS-SSO4-ED4 }\end{array}$ & N/A & $1.4 E+\infty 2$ & No \\
\hline Copper & $5 / 5$ & $\mathrm{~J} .1 \mathrm{E}+01$ & $3.0 \mathrm{E}+0 \mathrm{t}$ & $\begin{array}{l}\text { NS-SSO1-ED4 NS-SSO2-ED4 } \\
\text { NS-SSO3-ED4 NS-SSQ4-ED4 }\end{array}$ & NA & $3.1 \mathrm{E}+02$ & No \\
\hline fron & $5 / 5$ & 1.7E+04 & $2.6 \mathrm{E}+04$ & $\begin{array}{l}\text { NS-\$SO1-ED4 NS-SS02-ED4 } \\
\text { NS-SSO3-ED4 NS-SS04-ED4 }\end{array}$ & $N A$ & $23 \mathrm{E}+00$ & Yes \\
\hline Lead & $\ddot{5} / 5$ & $1.2 \mathbf{E}+0]$ & $3.1 E+01$ & $\begin{array}{l}\text { NS-SSOI-EDA NS-SS02-ED4 } \\
\text { NS-SSO3-ED4 NS-SSO4-ED4 }\end{array}$ & $N / A$ & $4.6 \mathrm{E}+02$ & No \\
\hline Mingesium & $5 / 5$ & $9.5 \mathrm{E}+02$ & $3.2 \mathrm{E}+1 \mathrm{l}$ & $\begin{array}{l}\text { NS-SSOL-EDA N5-S\$O2-ED4 } \\
\text { NS-SSO3-ED4 NS-SSO4-ED4 }\end{array}$ & $N / A$ & $\mathrm{~N} / \mathrm{A}$ & $N / A$ \\
\hline Manzpanege & $3 / 5$ & 1. $\pi \mathrm{E}+02$ & $9.7 \mathrm{E}+\overline{02}$ & $\begin{array}{l}\text { N\$S-\$SOI-EDA NS-\$SO2-ED4 } \\
\text { NS-S503-ED4 NE-SS04-ED4 }\end{array}$ & $N / A$ & $1.8 \mathrm{E}+102$ & Yes \\
\hline Mencury & $5 / 5$ & $5.3 \mathrm{E}-02$ & 8.5E- 02 & $\begin{array}{l}\text { NS-S501-EDA NS-SSO2-ED4 } \\
\text { NS-SSO3-ED4 NS-SSO4-ED4 }\end{array}$ & $\mathrm{ko}$ & $2.3 \mathrm{E}+00$ & No \\
\hline Nicket & $5 \sqrt{5}$ & $6.8 \mathrm{E}+00$ & d. JE+[k] & $\begin{array}{l}\text { NS-SS01-ED4 NS-SS02-ED4 } \\
\text { NS-S\$03-ED4 NS-SSO4-ED4 }\end{array}$ & $N / A$ & $1.6 E+02$ & No \\
\hline Potassiurs & $5 / 5$ & $6.8 \mathrm{E}+02$ & $1.7 \mathrm{E}+03$ & $\begin{array}{l}\text { NS-SS0]-ED4 NS-SSO2-EDA } \\
\text { NSS-SS03-ED4 NS-SSO4-EDN4 }\end{array}$ & $\mathrm{N} / \mathrm{A}$ & N/A & $N / A$ \\
\hline
\end{tabular}


Table 6.10. Parcel ED-4 study area soil - metals results (continned)

\begin{tabular}{|c|c|c|c|c|c|c|c|}
\hline Anabysto type & $\begin{array}{c}\text { Frequency } \\
\text { of } \\
\text { dutections }\end{array}$ & $\begin{array}{l}\text { Minlaium } \\
\text { conctintralion } \\
\text { (mojeg) }\end{array}$ & 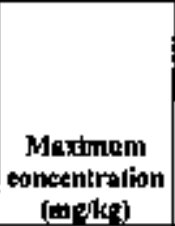 & $\begin{array}{l}\text { Losationis wherc } \\
\text { detected }\end{array}$ & $\begin{array}{c}\text { Exceeds } \\
\text { Zont I } \\
\text { ramedistion } \\
\text { levat? }\end{array}$ & $\begin{array}{c}\text { Retident } \\
\text { sodl } \\
\text { prellminsiny } \\
\text { remediation } \\
\text { sod } \\
\text { (molkg) }\end{array}$ & $\begin{array}{c}\text { Maximam } \\
\text { detect } \\
\text { exceeds } \\
\text { prebimlary } \\
\text { ramedialion } \\
\text { gonl } \\
\text { ? }\end{array}$ \\
\hline Seleninm & $5 / 5$ & $4.0 E+01$ & $9.5 \mathbf{E}+61$ & $\begin{array}{l}\text { NS-SS01-ED4 NS-SS02-EDA } \\
\text { NS-SSO3-ED4 NS-SSO4-ED4 }\end{array}$ & $\mathrm{N} / \mathrm{A}$ & $3.9 \mathrm{E}+0 \mathrm{O}$ & Yes \\
\hline Silicon & $5 / 5$ & $1.0 E+03$ & $1.3 E+03$ & $\begin{array}{l}\text { NS-SSOl-ED4 NS-SSO2-ED4 } \\
\text { NS-SSO3-ED4 NS-SSO4-ED4 }\end{array}$ & $\mathrm{N} / \mathrm{A}$ & $N / A$ & $\mathbf{N} / \mathbf{A}$ \\
\hline Sעver & $3 / 5$ & $35 \mathrm{E}+00$ & $4.8 \mathrm{E}+100$ & NS-SSOL-ED4 NS-SSOZ-ED4 & $\mathrm{N} / \mathrm{A}$ & $3.9 \mathrm{E}+0 \mathrm{~J}$ & $\mathrm{~N} 0$ \\
\hline Sodiuma & $5 / 5$ & ]企+01 & $5.4 \mathrm{E}+01$ & $\begin{array}{l}\mathrm{NS} \text {-SSOI-ED4 NS-SSO2-ED4 } \\
\mathrm{NS} \text {-S5O3-ED4 NS-S504-ED4 }\end{array}$ & $\mathrm{N} / \mathrm{A}$ & $\mathbf{N} / \mathbf{A}$ & $\mathrm{N} / \mathrm{A}$ \\
\hline Thallisen & $0 / 5$ & $N / A$ & N/A & & $\mathbf{N} / \mathbf{A}$ & $5.2 \mathrm{E}-01$ & $\mathrm{~N} / \mathrm{A}$ \\
\hline Vartaxtitun & $5 / 3$ & $2.3 \mathrm{E}+01$ & $3.6 \mathrm{E}+01$ & $\begin{array}{l}\text { NS-SSO1-ED4 NS-SSD2-ED4 } \\
\text { N5-SS03-ED4 NS-SS04-EDL4 }\end{array}$ & N/A & $7.8 \mathrm{E}+00$ & Yes \\
\hline Žink & $5 / 5$ & $2.0 \mathrm{E}+01$ & $6.0 \mathrm{E}+0 \mathrm{I}$ & $\begin{array}{l}\text { NS-5501-ED4 NS-5S02-ED4 } \\
\text { NS-5503-ED4 NS-5S04-ED4 }\end{array}$ & $\mathrm{N} / \mathrm{A}$ & $2.3 \mathrm{E}+013$ & No \\
\hline
\end{tabular}

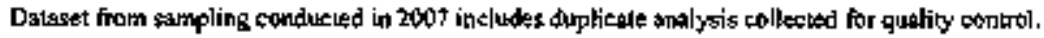

Residential soil PRG at a risk level of $L E-06$ and a bazard index of 0.1 .

$\mathrm{mg} / \mathrm{kg}$ - milligrams chemizal kilogrant soil.

NAA = nol available.

\section{Asbestos}

Asbestos was analyzed in selected surface soil samples collected at ED-4. A total of three (3) abbestos samples and one (1) dupilicate sample were collected from surface soile and submitted for laboratory analysis. The samples analyzed for abbeatos ineluded the SS01-ED4, SS02-ED4, and SSO4-ED4 locations. A duplicate sample was collected from the \$SOI-ED4 location. All sample anaiysis results for the surface soil samples were negative for the presence of asbestos.

\subsubsection{Chemical Sampling Results - Groundwater}

\subsubsection{Groundwater results analysis}

In accordance with the approved SAP for Land Parcel ED-4, groundwater samples were to be collected using temporary drive-point piezometers and existing monitoring wells. Five temporaty piezometers were installed for the purpose of groundwater sampie collection at ED-4. Installation took place on July 19, 2007. Locations of the ternporary piezometers and the existing monitoring wells in the vicinity of ED-4 ane indicated on Fig. 6.2. Tabie6.11 indicates the total depths of the completed piezometers and existing wells sampled at ED-4, the formation monitored by the piezometers and weils, the date sampie collection was attempted, and whether or not a sampie was suecessfully collected.

The collection of groundwater samples from the drive-point piezorneters was attempted on July 23, 2007. On that date, four of the five piezometers were dry [NS-01-GW-ED4 (18.5 ft bgs), NS-03-GWED4 (12.9 ft bgs), NS-04-GW-ED4 (10.1 ft bgs), and NS-05-GW-ED4 (9.29 ft bgs)]. The piezometers were checked periotically for water leveis, and a groundwater sample was collected from piczorneter $\mathrm{NS}-02-\mathrm{GW}-\mathrm{ED} 4(12.8 \mathrm{ft}$ bgs) on July 30,2007 . In addition to four of the five drive-point piezometers, one existing upgradient monitoring well (UNW-102, $31.5 \mathrm{ft}$ bgs) was also found to be dry during the

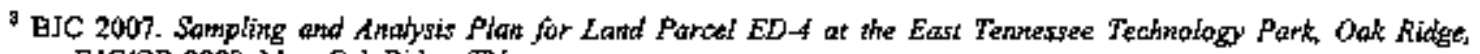
Ternessee, BJCfOR-2809, Mey, Oak Ridge, TN. 
period that sample collection was attempted. Well UNW-102 continued to be dry on the date that the paired well, BRW-076 (60.5 ft bgs), was sampled on July 30, 2007. The side-gradient unconsolidated zone well (UNW-125, $20 \mathrm{ft}$ bgs) was sampled on August 2, 2007. Groundwater samples collected fromi the bedrock well BRW-076 were analyzed for herbicides, pesticides, metals, VOCs, SVOCs, and radiological paraneters. Groundwater samples collected from well UNW-125 wete analyzed for herbicides, pesticides, metals, VOCs, and radiological parameters. Groundwater samples collected from temporary piezometer 02 were analyzed for herbicides, pesticides, metals, VOCs, and radiological parameters. Samples for SVOC analysis were not collected at UNW-125 and the temporary piezometer 02 due to the limited water volume obtained from these shallow monitoring loctations. The temporary piezometer samples were not filtered at the time of collection, and due to questionable radiological results, subsequent analyses for radiological parameters were conducted on a subsample filtered in the laboratory. The results of the radiological re-analysis are discussed below in Sect. 6.2.2.4. Figure 6.2 indicates the piezometer and well locations that were dry and sumnarizes the analytical results for VOCs in groundwater samples collected at Parcel ED-4.

Table 6.11. Groundwater sample collection locatlons for Parcel ED-4

\begin{tabular}{|c|c|c|c|c|}
\hline Location & Formation monitored & $\begin{array}{c}\text { Total } \\
\text { depth (ft) }\end{array}$ & $\begin{array}{l}\text { Date sampling } \\
\text { attempted }\end{array}$ & $\begin{array}{c}\text { Date } \\
\text { sample } \\
\text { collepted }\end{array}$ \\
\hline \multirow{4}{*}{ NS-01-GW-ED4 } & Inconsolidated zone & 18.5 & $7 / 19 / 07$ & NA \\
\hline & & & $7 / 23 / 0 \mathrm{~T}$ & \\
\hline & & & $7 / 24 / 07$ & \\
\hline & & & $7 / 30 / 07$ & \\
\hline \multirow[t]{4}{*}{ NS-02-GW-ED4 } & Unconsoljdated zone & 12.8 & $7 / 19 / 07$ & $7 / 30 / 07$ \\
\hline & & & $7 / 23 / 07$ & \\
\hline & & & $7 / 24 / 07$ & \\
\hline & & & $7 / 30 / 07$ & \\
\hline \multirow[t]{4}{*}{ NS-03-GW-ED4 } & Unconsolidated zorite & 12.9 & $7 / 9 / 07$ & NA \\
\hline & & & $7 / 23 / 07$ & \\
\hline & & & $7 / 24 / 07$ & \\
\hline & & & $7 / 30607$ & \\
\hline \multirow[t]{4}{*}{ NS-04-GW-ED4 } & Unconsolidated zone & 10.1 & $7 / 19 / 07$ & NA \\
\hline & & & $7 / 23 / 07$ & \\
\hline & & & $7 / 24 / 07$ & \\
\hline & & & $7 / 30 / 07$ & \\
\hline \multirow[t]{4}{*}{ NS-05-GW-ED4 } & Unconsolidated zone & 9.29 & $7 / 19 / 07$ & $\mathrm{NA}$ \\
\hline & & & $7 / 23 / 07$ & \\
\hline & & & $7 / 24 / 07$ & \\
\hline & & & $7 / 30 / 07$ & \\
\hline BRW-076 & Bedrock & 60.5 & July 30,2007 & $7 / 30 / 07$ \\
\hline \multirow[t]{4}{*}{ UNW-102 } & Unconsolidated zone & 31.5 & $7 / 19 / 07$ & NA \\
\hline & & & $7 / 23 / 0 ?$ & \\
\hline & & & $7 / 24 / 07$ & \\
\hline & & & $7 / 30 / 07$ & \\
\hline UNW-125 & Unconsolidated zone & 20.0 & August 2, 2007 & $8 / 01 / 07$ \\
\hline
\end{tabular}

NA - Groundwater sample could not be collecled due to insufficient water. 


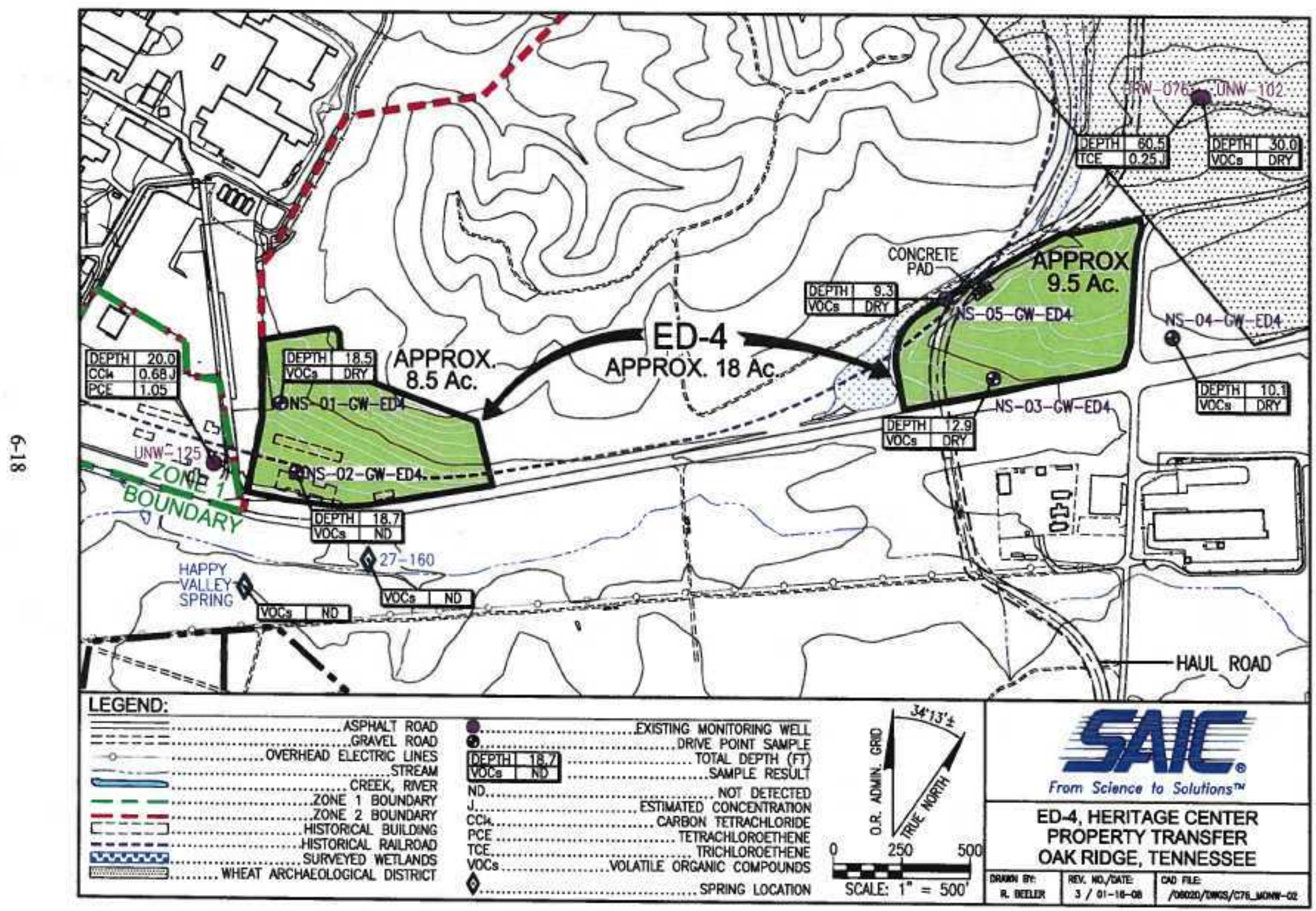

Fig. 6.2. Groundwater sampling locations and results for VOCs at ED-4. 
Tabie 6.12 provides a sumunary of the sample collection history and analytical results for VOCs, including July 2007 results, for the existing wells in the vicinity of ED-4. Tabie 6.12 inciudes the wetl identification, the date sampled, the VOCs detected for each sampling event, and the concentration of the VOCs detected. VOCs had not been detected al the upgradient bedrock well BRW-076 in any bistorical sampling results untit the July 2007 sample collection event. Trichloroethene (TCE) was detected at an estimated concentration of $0.25 \mu \mathrm{g} / \mathrm{L}$ at well BRW -076 . However, due to the absence of TCE in hislotical samples and the low concentration reported in July 2007, this result may be questionable and is wetl below the MCL of $5 \mu \mathrm{g} / \mathrm{L}$ established for this compound."

Table 6.12. Anglytical results for VOCs in grougdwater at Parcel ED-4

\begin{tabular}{|c|c|c|c|}
\hline Well & Date sampled & VOCs detected & Concentration $(\mu \sigma L)$ \\
\hline \multirow{6}{*}{$\begin{array}{l}\text { BRW-076 } \\
\text { upgradien! }\end{array}$} & September 1994 & $\mathrm{ND}$ & NA \\
\hline & February 1995 & ND & NA \\
\hline & September 1995 & $\mathrm{ND}$ & NA \\
\hline & February 2005 & $\mathrm{ND}$ & NA \\
\hline & March 2005 & ND & NA \\
\hline & July 2007 & Trichlorothene & $0.25 \mathrm{~J}$ \\
\hline \multirow{6}{*}{$\begin{array}{l}\text { UNW-102 } \\
\text { upgradient }\end{array}$} & September 1994 & $\begin{array}{c}\text { Toluene } \\
\text { 1,1,1-Trichloroethane }\end{array}$ & $\begin{array}{l}38 \\
2 \mathrm{~J}\end{array}$ \\
\hline & February 1995 & ND & NA \\
\hline & Sefptember 1995 & 2-Butanone & 10 \\
\hline & December 2004 & $\mathrm{ND}$ & NA \\
\hline & March 2005 & $\begin{array}{c}\text { 1,1,1-Trichloroethane } \\
\text { 1,1-Dichloroethane } \\
\text { 1,I-Dichloroethene }\end{array}$ & $\begin{array}{l}0.56 \mathrm{~J} \\
0.33 \mathrm{~J} \\
0.19 \mathrm{~J} \\
\end{array}$ \\
\hline & July 2007 & Dry- no sample collected & $\mathrm{NA}$ \\
\hline \multirow{5}{*}{$\begin{array}{l}\text { UNW-125 } \\
\text { side-gradient }\end{array}$} & April 1998 & $\begin{array}{c}\text { 1,1,2-Trichloro-1,2,2-trifluoroethane } \\
\text { Acetone } \\
\text { Toluene } \\
\text { Trichloroethene }\end{array}$ & $\begin{array}{l}8 \\
5 \mathrm{~J} \\
1 \mathrm{~J} \\
3 \mathrm{~J}\end{array}$ \\
\hline & September 1999 & Methylene chloride & $4 y$ \\
\hline & February 2000 & ND & NA \\
\hline & August 2000 & ND & NA \\
\hline & August 2007 & $\begin{array}{l}\text { Carbon tetrachloride } \\
\text { Tetrachlorocthene }\end{array}$ & $\begin{array}{l}0.68 \mathrm{~J} \\
1.05\end{array}$ \\
\hline $\begin{array}{l}\text { NS-012-GW-EV4 } \\
\text { downgradient }\end{array}$ & July 2007 & $\mathrm{ND}$ & NA \\
\hline
\end{tabular}

BRW - bedrock wrell.

GW $=$ grouthwater.

$\mathrm{J}=$ estimated concentration.

NA - not applicsble.

$\mathrm{ND}=$ Thot detected.

UNW = unconsolidated zone well.

VOC = volatile organic compourd.

Carton tetrachloride and tetrachloroethene were detected at side-gradient well UNW-125 at low concentrations (0.68 J and $1.05 \mu \mathrm{g} / \mathrm{L}$, respectively) during the July sampling event. However, atthough VOCs have been reported sporadically at this side-gradient wetl, none of the reported compounds have

\footnotetext{
${ }^{9}$ Maximum contamitianl levels (MCLs) for drinking water have been eslablished by the EPA

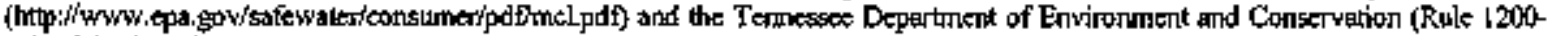
1-15-, (66, 12, and 25). Comparisons to MCLs are made to be consistest wilh EM"'s groundwater progsam at ETTP.
} 
been detected in more than ont sampling event at this well. The repported concentrations of carbon tetrachloride and tetrachloroethene at well UNW-125 during the July 2007 sampling event do not exceed the MCL of $5 \mu \mathrm{g} / \mathrm{L}$ established for these compounds. ${ }^{\text {to }}$

VOCs have been reponted during three of five historical sampling events at the upgradient unconsolidated zone monitoring well UNW-102, which was dry during the attempted sampling event in

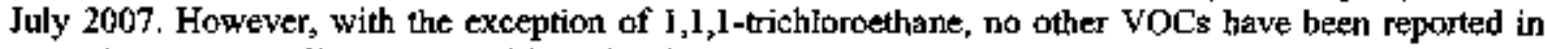
more than one sampling event at this well. The VOC $1,1,1$-trichloroethane was reported at low estimated concentrations in the 1994 and 2005 sampling events.

Analytical resutts for the single temporary piezometer (NS-02-GW-E04) that was not dry during the Juty 2007 sampling event indicate that no VOCs are present in groundwater at this location.

Herbicides and pesticides were not detected in groundwater samples collected from the existing wells (BRW-076 and UNW-125) and the temporary piezoneter (02) at Parcel ED-4. In addition, SVOCs were not detected in the sample where analyzed for at bedrock well BRW-076.

The analybical results for metals are summarized in Teble 6.13. This table also includes background concentrations established for ETTP for the Sitewide Remedial Investigation (DOE 2007b) and applicable MCLs. Metals exceeding established MCLs were only detected in groundwater samples collected from the temporary piezometer (02) installed at ED-4. However, samples collected from this piezometer were not fittered prior to preservation of the sample, and significant suspended solids were present in the sample obtained from the piezorneter. The required acid preservation of water samples may release thetals bound to the surface of the suspended solids or dissolve some of the solids altogether, thus resulting in higher metals concentrations in the sample (Giles and Story 1997). There were no exceedances of MCLs in the groundwaler semples collected from the existing monitoring wells.

T*ble 6.13. Analytical results for metals in groundwater at Parcel ED-4

\begin{tabular}{|c|c|c|c|c|c|}
\hline \multirow[b]{2}{*}{ Analyte } & \multirow[b]{2}{*}{ Background } & \multirow[b]{2}{*}{ MCL } & \multicolumn{3}{|c|}{ Well identification } \\
\hline & & & $\begin{array}{c}\text { BRW-076 } \\
\text { (topperadient) }\end{array}$ & $\begin{array}{c}\text { UNW-125 } \\
\text { (side-gradlient) }\end{array}$ & $\begin{array}{c}\text { Temporary } \\
\text { plezometer } \\
02\end{array}$ \\
\hline Aluminum & $1.0 \mathrm{~J} \widehat{\mathrm{B}}$ & & 1.030 & $0.049 \mathrm{U}$ & 420 \\
\hline Antimory & 0.00095 & 0.006 & $0,008 \mathrm{U}$ & $0.008 \mathrm{U}$ & $\mathrm{NA}$ \\
\hline Arsentic & 0.0019 & 0.01 & $0.007 \mathrm{U}$ & $0.007 \mathrm{U}$ & 0.0961 \\
\hline Baium & 0.249 & 2 & 0.197 & 0.122 & 2.89 \\
\hline Beryllium & 0.00025 & $0,0,04$ & $0.0002 \mathrm{U}$ & $0.0002 \mathrm{U}$ & 0.0381 \\
\hline Boron & 0.0252 & & $0.025 \mathrm{~J}$ & 0.0235 & $0.265 \mathrm{~J}$ \\
\hline Cadmium & 0.0016 & 0.005 & $0.001 \mathrm{U}$ & $0.001 \mathrm{U}$ & $0.005 \mathrm{U}$ \\
\hline Caicium & 72.5 & & 30.9 & 101 & 642 \\
\hline Chromium & 0.011 & 0.1 & 0.016 & $0.0049 \mathrm{~J}$ & 0.435 \\
\hline Cobalt & 0,0067 & & $0.0019 \mathrm{U}$ & $9.001 \mathrm{U}$ & 0.303 \\
\hline Coppes & 0.007 & 1.3 & $0.106 \mathrm{~J}$ & 0.1111 & 0.474 \\
\hline Iron & 1.63 & & 1.9 & $0.054 \mathrm{U}$ & $\$ 45$ \\
\hline Lead & 0.0045 & 0.015 & $0.004 \mathrm{U}$ & $0.004 \mathrm{U}$ & 0.537 \\
\hline Magnesium & 24.7 & & 10.2 & 22.5 & 98.5 \\
\hline Manganese & 0.14 & & 0.268 & $0.009 \mathrm{~J}$ & 24.4 \\
\hline
\end{tabular}

10 Jbid. 
Table fi.13. Analytical results for metals in groundwater at Parcel ED-4 (continued)

\begin{tabular}{|c|c|c|c|c|c|}
\hline \multirow[b]{2}{*}{ Analyte } & \multirow[b]{2}{*}{ Background } & \multirow[b]{2}{*}{ MCL } & \multicolumn{3}{|c|}{ Wel identícation } \\
\hline & & & $\begin{array}{c}\text { BRW-076 } \\
\text { (eparadient) }\end{array}$ & $\begin{array}{c}\text { LNW-125 } \\
\text { (stde-eradlent) }\end{array}$ & $\begin{array}{c}\text { Temaporary } \\
\text { piezometer } \\
02\end{array}$ \\
\hline Mercury & 0.00013 & 0.002 & $0.00003 \mathrm{UJ}$ & $0.00003 \mathrm{U}$ & $0.0016 \mathrm{~J}$ \\
\hline Nickel & 0.023 & & 0.204 & $0.0031 \mathrm{~J}$ & 0.627 \\
\hline Potassium & 5.62 & & 4.03 & 1.38 & $58.2 \mathrm{~J}$ \\
\hline Selenium & 0.0012 & 0.05 & $0.005 \mathrm{~J}$ & 0.0050 & $0.0428 \mathrm{~J}$ \\
\hline Silicon & 15.7 & & 18.8 & 2.16 & 111 \\
\hline Silver & 0.00023 & & $0.002 \overline{\mathrm{U}}$ & $0.002 \mathrm{U}$ & $0.002 \mathrm{U}$ \\
\hline S̃odium & 12.06 & & 19,1 & 3.24 & 2.91 \\
\hline Thallium & 0.0021 & 0.002 & $0,008 \mathrm{U}$ & $0.008 \mathrm{U}$ & $0.04 \mathrm{U}$ \\
\hline Vanadium & 0,008 & & $0,0021 \mathrm{~J}$ & $0.002 \mathrm{U}$ & 0.475 \\
\hline Zinc & 0.032 & & $0.102 \mathrm{~J}$ & $0.918 \mathrm{~J}$ & 1.5 \\
\hline
\end{tabular}

Note: All concentratious are in metL

ERW = bedrock motritoring well.

$\mathrm{J}$ w estimated concentration.

MCL = maximum contaminant level.

$U=$ not derected at or above indicated concentration.

UJ = aot detected at or above indicated concentration and concentration is estimated.

UNW - unconsolidared monitoring we]].

Concentrations of metals in existing monitoring wells that exceeded groundwater background concentrations established for $\mathrm{ETTP}^{1 \mathrm{l}}$ include aluminum, calcium, chromium, copper, iron, manganese, nickel, silicon, sodium, and zinc. However, only copper, nickel, and zinc were detected at concentrations excedding the background criteria, with all other metals below their background criteria. The elevated concentrations of copper, nickel, and zinc may be, in part, the to the unfiltered samples collected from these wells and the fact that these wells have not been re-developed since their installation in 1993 (BRW-076) and 1998 (UNW-125).

The groundwater sampte collected from the temporary piezometer 02 indicates that MCLs for several metals were exceeded. The metals exceeding MCLs included arsenic, barium, beryllium, chromium, lead, and mescury. However, as mentioned previously, this sample was unfiltered and the acid preservation of the sample, which contained a significant suspended solids content, likely has impacted the results from this location (Giles and Story 1997).

Due to the uncertainty of the results for the ED-4 groundwater samples obtained from the temporary piezometer, discussions with EPA were held in November 2007 to determine an appropriate path forward with respect to groundwater at ED-4. Based on discussions with the EPA, it was agreed that groundwater quality results from springs located across Highway 58 on Parcel ED-3 could be used as being representative of groundwater quality discharging from ED-4. Two springs located within Parcel ED-3 have been sampled more than once as noted below. These include the Happy Valley Spring and spring 27-160 (Fig. 6.2).

TDEC conducts routine sampling of selected springs around the ORR, including the Happy Valley Spring, which is located approximately $300 \mathrm{ft}$ south of the western tract of ED-4. Analytical results for the period from 1998 through 2002 are available for samples collested from this spring. Samples from this spring, which represents a discharge point for groundwater on the south side of Higbway 58

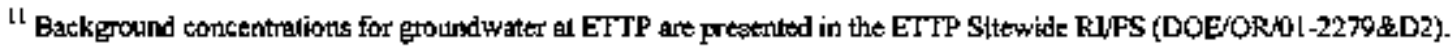


(Oak Ridge Tumpike), have been analyzed for radiological parameters, metals, VOCs, and general water quality parameters (e.g, alkalinity, chloride, mitrate/nitrite, and stifate). The results for the chemical constituents are summarized in Table 6.14.

Table 6.14. Summary of analytutal resulfs for the Happy Valley Spring

\begin{tabular}{|c|c|c|c|c|c|}
\hline Anglyte & $\begin{array}{l}\text { Frequency of } \\
\text { deterts }\end{array}$ & $\begin{array}{l}\text { Minimum } \\
\text { detected } \\
\text { concentration }\end{array}$ & $\begin{array}{l}\text { Maximum } \\
\text { defected } \\
\text { concentration }\end{array}$ & $\begin{array}{c}\text { Drinking } \\
\text { water MCL } \\
(\mu g / \mathrm{L})\end{array}$ & $\begin{array}{c}\text { Frequency } \\
\text { of detects } \\
\text { exceedies } \\
\text { MCL }\end{array}$ \\
\hline Arsenic $(\mu g / L)$ & $2 / 9$ & 1.0 & 1.1 & 10 & $0 / 2$ \\
\hline Cadmium $(\mu g / L)$ & o/s & NA & NA & 5 & NA \\
\hline Chromium ( $\mu \mathrm{g} / \mathrm{L})$ & $3 / 8$ & 1.0 & 1,0 & 100 & $d / 3$ \\
\hline Iron $(\mu, \alpha)$ & I/] & 113 & 113 & $300^{2}$ & NA \\
\hline$\overline{\mathrm{L}} \mathrm{ead}(\mu \mathrm{g} / \mathrm{L})$ & $1 / 8$ & 1.0 & 1.0 & 15 & $\alpha^{\prime} 1$ \\
\hline Magnesium $(\mu \mathrm{g} \mathrm{L})$ & $1 / 1$ & 20,300 & 20,300 & $\mathrm{NA}$ & NA \\
\hline Mercury ( $\mu g / L)$ & ors & NA & NA & 2 & $N A$ \\
\hline Nickel $(\mu \mathrm{g} / \mathrm{L})$ & $1 / 4$ & 14 & 14 & NA & NA \\
\hline Seletium $(\mu q / \mathrm{L})$ & of 8 & NA & NA & 50 & NA \\
\hline Thallium ( $\mu \mathrm{s} / \mathrm{L})$ & $0 / 8$ & NA & NA & 2 & NA \\
\hline $\operatorname{Zinc}(\mu g / \mathrm{L})$ & $8 / 8$ & 2 & 8 & $5,000^{7}$ & $\mathrm{NA}$ \\
\hline VOCs $(\mu \mathrm{g} / \mathrm{L})$ & $0 / 8$ & $\overline{N A}$ & NA & $\mathrm{NA}^{*}$ & NA \\
\hline
\end{tabular}

"Represents secondary nen-enforceable drinking water standard.

$\mathrm{MCL}$ = maximutn contarninant level.

$N A=$ not applicable.

VOCs = volatile organic compounds.

* MCLs apply only to specific VoCs.

Based on the resuits for the Happy Valiey Spring, no metais or VOCs have been detected above an MCL. Thus, there is no evidence of contamination in groundwater at this spring.

Spring 27-160 was identified by the U. S. Geological Survey (USGS) during a spring and seep survey of the ETTP conducted in 1994 and 1995 . Samples were collected from this spring to support the Groundwater Remedial Site Evaluation Report for ETTP (DOE 1996) in 1995. The analytical results for samples collected in April and October of 1995 at the 27-160 spring, located approximately $450 \mathrm{ft}$ northeast of the Happy Valley Spring, are summarized in Table 6.15.

Results for samples collected from the $27-160$ spring indicate there is no evidence of groundwater contarnination at this spring. No organic compounds were detected. All conceutrations of the only metal detected that has a corresponding MCL (barium) were well below the MCL.

In addition, groundwater samples have been collected from two temporary piezorneters installed within the adjacent and downgradient Parcel ED-3. One piezometer was located approximately $200 \mathrm{ft}$ worth of the Happy Valley Spring and the second piezometer was located on the south side of Highway 58, approximately halfway between the two ED-4 tracts. Results for these piezometers indicate that the only VOCs detected have been benzene (maximum of $1.36 \mu \mathrm{g} / \mathrm{L}$ ) and xylene (maximum of $1.02 \mu \mathrm{g} / \mathrm{L}$ ). The detected concentrations are below the MCLs for these compounds. 
Table 6.15. Summary of analytical results for Spring 27-160

\begin{tabular}{|c|c|c|c|c|c|}
\hline Constituent & $\begin{array}{c}\text { Frequency of } \\
\text { detects }\end{array}$ & $\begin{array}{l}\text { Mininum } \\
\text { detected } \\
\text { concentration }\end{array}$ & $\begin{array}{l}\text { Maximum } \\
\text { detected } \\
\text { concentretion }\end{array}$ & $\begin{array}{c}\text { Drinklng } \\
\text { water MLL } \\
\text { (1g/L) }\end{array}$ & $\begin{array}{c}\text { Frequency } \\
\text { of detects } \\
\text { exceeding } \\
\text { MCL }\end{array}$ \\
\hline Arșenjc $(\mu \mathrm{g} / \mathrm{L})$ & $0 / 4$ & $\mathrm{NA}$ & NA & 10 & NA \\
\hline Barium $(\mu \mathrm{g} / \mathrm{L})$ & $4 / 4$ & 46.3 & 49.5 & 2000 & $0 / 4$ \\
\hline Cadmium $(\mu \alpha / L)$ & $0 / 4$ & $\overline{\mathrm{AA}}$ & NA & 5 & $\overline{N A}$ \\
\hline Chromiuts $(\mu / L)$ & $0 / 4$ & NA & $N A$ & 100 & $\mathrm{NA}$ \\
\hline Lead $(\mu g / L)$ & $0 / 2$ & $\overline{\mathrm{NA}}$ & NA & 15 & $\overline{N A}$ \\
\hline Selenium (kgL) & $0 / 4$ & NA & $\mathrm{NA}$ & 50 & NA \\
\hline Thallium ( $\mu$ gL) & $0 / 4$ & $\mathrm{NA}$ & NA & 2 & $\overline{N A}$ \\
\hline $\operatorname{VOCs}(\mu g / L)$ & $0 / 2$ & NA & NA & $\mathrm{NA}^{*}$ & $\mathrm{NA}$ \\
\hline SVOCS $(\mu \mathrm{gLL})$ & $\overline{0 / 2}$ & NA & $\mathrm{NA}$ & $\mathrm{NA}^{*}$ & $N A$ \\
\hline Herbicides ( $\mu \mathrm{g} / \mathrm{L})$ & $\mathrm{O} / 2$ & $\mathrm{NA}$ & $\mathrm{NA}$ & $\mathrm{NA}^{*}$ & $\mathrm{NA}$ \\
\hline Pesticides ( $\mu \mathrm{g} / \mathrm{L})$ & $0 / 2$ & $\mathrm{NA}$ & NA & $\mathrm{NA}^{*}$ & $\mathbf{N A}$ \\
\hline PCBs $(\mu \mathrm{g} / \mathrm{L})$ & $0 / 2$ & $\mathrm{NA}$ & $\mathrm{NA}$ & $\mathrm{NA}^{*}$ & $\mathrm{NA}$ \\
\hline
\end{tabular}

* MCLs are only epplicable to specific compounds.

MCL = maximum conlaminant level.

$N A$ - not applicable.

PCBs = polychlorinated biphenylg.

SVOCs $=$ semivolatile organic compounds.

VOCs * volatile organic compounds.

Based on discussions with the EPA, groundwater data collected at springs and temporary piezometers located south and downgradient of ED-4 (in ED-3) have been included in this report to support a CPD. The results of these groundwater samples and those collected at existing monitoring wells and temporary piezometers within Parcel ED-4 indicale that there is no evidence of groundwater contamination from chemical constituents at this parcel.

\subsection{RADIOLOGICAL SURVEYS AND SAMPLING}

This section presents and discusses both historical and recent radiological survey data and the radiological soil sampling results collected from the study area. The availability of historical survey data is discussed, followed by a discussion of the survey methodology and results used to supplement the historical data. The final subsection presents a sumomary of the 2007 soil sample data.

The process history of the ETTP indicates uranium (natural, depleted, and/or enriched) is the most prominent radiological contaminant potentially present in the ED-4 land parcel as a result of tracking contamination from on-site buildings or from environmental release. Uranium-235 enrichment levels of contamination from operations since the early $1960 \mathrm{~s}$ are expected between 0.2 and $5.0 \%$. Most on-site facilities were potentially contaminated from enrichrnents of less than $3 \%{ }^{12}$

Other radionuclides detected on-site at ETTP are ${ }^{60} \mathrm{Co},{ }^{197} \mathrm{Cs},{ }^{8990} \mathrm{Sr}_{2}{ }^{257} \mathrm{~Np},{ }^{99} \mathrm{Tc}$, and ${ }^{258239240} \mathrm{Pu}$. These other tadionuclides originated from the introduction of contaninated materials from Oak Ridge National Laboratory andior from the Hanford and Savannah River reactor returns uratnium-reprocessing program.

\footnotetext{
${ }^{12}$ Contracted Health Physics Technieien Training handouts, K-25 Sitt, 1993.
} 


\subsubsection{Radjological Surveys}

\subsubsection{Eistorical surveys}

No historical radiological survey data were found for this land parcel. However, the eastern tract is bisected by DOE's Haul Road. The Haul Road is being surveyed monthly using a large-area plastic scintillator (LAPS) detector that can be compared to the established management limits. It will be assumed that any contamination identified in connection with monitoring the Haul Road will be remediated prior to transfer; Haul Road activities are outside the scope of this survey plan.

\subsubsection{2007 Exterior sarwey units}

As the survey teams were performing the land survey units (LSUs) walkover scans, a concrete pad and other concrete debris were idenlified in ED-4. The pad was probably an old building foundation and measured approximately $36 \mathrm{ft}$ by $80 \mathrm{ft}\left(2880 \mathrm{ft}^{2}\right)$. The pad was surveyed with the sodium iodide (NaI) probe in order to detect elevated readings. None were detected. The pad was classified as a Class 3 exterior survey unit (ESU) for further survey.

One exterior radiological surface survey was conducted (survey number 07 -AREAC-1377) for the concrete pad. The survey was performed on May 18, 2007, in accordance with ETTP Radiation Control (RADCON) procedures ${ }^{13}$ and the survey design document ${ }^{\text {t4 }}$ (hereafter referred to as the "design document") and the survey plan (see Appendix E). The concrete pad was scan-surveyt over the accessible area that was not covered by moss, weeds, or vegetation (available pad area was less than $20 \mathrm{ft}^{2}$ ). The accessible locations for survey were isolated to multipie small areas of exposed concrete across the entire surface of the pad. Twenty-one measurements of total and removable activity were taken (alphe and betaugamma) at locations having the highest activities, as determined during the scan survey. A summary of the survey results is shown in Table 6.16.

All readings taken in the ESU were less than $1250 \mathrm{dpm} / 100 \mathrm{~cm}^{2}$ total activity. Because all results were well below the respective screening levels, no further statistical analysis was performed. Based on an inspection of the individual surveys, including quality assurance/quality control (QAVC) surveys, ali total activities were less than $24 \mathrm{dpm} / 100 \mathrm{~cm}^{2}$ total alpha and $375 \mathrm{dpm} / 100 \mathrm{~cm}^{2}$ total beta-gamma, with alt removable contanination results less than $19 \mathrm{dpm} / 100 \mathrm{~cm}^{2}$ removable alpha and $56 \mathrm{dpm} / 100 \mathrm{~cm}^{2}$ removable beta-gamma The maximum tissue-equivalent dose rate was $\$$ prem/hour.

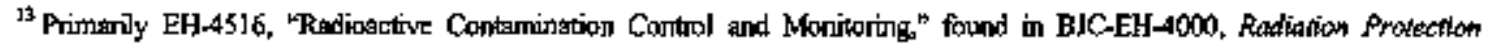
Prograpn Description for Bechtel Jacobs Compary LLC, Oak RLdge, Tentessee.

${ }^{14}$ Design of Radiological Survey and Sampling to Support Titte Tratisfer or Lectse of Property on the Department of Energy Oak Ridge Resernation, BJClOR-554-R1, Bechtel Jacobs Compay LLC, Gak Ridge, TN, Augusi 2006.
} 
Table 6.16. Summary of contomination and dose rates

\begin{tabular}{|c|c|c|c|c|c|c|c|c|c|c|c|c|c|c|c|c|c|}
\hline \multirow[b]{2}{*}{ Loration } & \multicolumn{4}{|c|}{ Ajphn total } & \multicolumn{4}{|c|}{ Alphz remorable } & & \multicolumn{3}{|c|}{ Beto-ganme totol } & \multicolumn{4}{|c|}{ Beta-ganma remuyable } & \multirow{2}{*}{$\begin{array}{c}\text { Dose equlvalent rate } \\
\text { (mrem/li) }\end{array}$} \\
\hline & & Min. & & Mox. & & Min. & & Hax. & & Min. & & Max. & & Mis. & & Max. & \\
\hline \multicolumn{18}{|c|}{ Esteribr surney ander - Closs 3} \\
\hline ESOI & 4 & 24 & 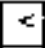 & 44 & $<$ & 19 & ए & 19 & 4 & 375 & $<$ & 375 & $<$ & 56 & 2 & 56 & 0.905 to 0.008 \\
\hline DOEE conlamsnatan limıs & & & & 50000 & & & & 1000 & & & & 5000 & & & & IOOÓ் & 20 \\
\hline
\end{tabular}

Noter. All readings we in units of disintegrations per miute $\left(\mathrm{dpm} / 100 \mathrm{~cm}^{2}\right.$.

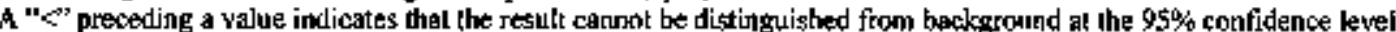

This table does not include results from quafily assurinceidquality controf sirveys.

DOE $=$ U. S. Department of Enaryy.

ESU = exlenior survey unit.

mrem/h = millirem per lsour 


\subsubsection{Land survey units}

A separate sampling plan was prepared to evaluate soil contamination and is discussed below. Surveys of the soils are conducted to identify possible additional sampling locations. Many of the radionuclides found on the ORR have natural background concentrations; therefore, background subtraction is required for all direct field measurements and laboratory analyses. Some comparison to background levels is required for the scanning because only a gross signal was measured

The ED-4 land parcel consisted of two LSUs, LSU 1 is the westem tract while LSU 2 is the eastern tract. Both ED-4 tracts were classified as Class 3.

A total of six radiological land surveys were conducted in the proposed transfer footprint The survey numbers are listed in Table 6.17. The surveys of the fixed-grid assessment points were performed from May 8, 2007, to May 14,2007 , and the surveys of the biased assessment points were performed on June 14, 2007, in accordance with ETTP RADCON procedures, ${ }^{2}$ the survey design document, ${ }^{3}$ and the survey plan (see Appendix E). Fixed messurements were made at the predeternined fixed-grid locations in the LSUs per Appendix E, Fig. E.2. Biased measurements were made at locations of anthropogenic disturbances or sediment accumulation areas determined from the walkover survey.

Table 6.17. Current ETTP landi radiological sarveys

\begin{tabular}{c}
\hline 07-AREAC-1416 \\
07-AREAC-1417 \\
07-AREAC-1418 \\
07-AREAC-1419 \\
07-AREAC-1420 \\
200706152AKDESK001 \\
\hline
\end{tabular}

$\mathrm{NeI}$ walkover survey scans were performed according to the survey plan (Appendix E). Emphasis was placed on roadbed areas, areas of soil or vegetation discoloration, and other areas based on professional judgment. NaI survey measurements were performed at the fixed-grid assessutent points; any anthropogenic, groundwater runoff, and sediment collection areas recognized during the NaI walkover; and any scan areas determined to have elevated readings. A 10 -ft-diameter surface area was to be scanued at each of these points with the NaJ detector, however, a 20 -ft-diameter surface area was actually scanned. The measurements at each point, to include only timed, 1-min-count $\mathrm{NaJ}$ and dose-rate measurements at the location of the highest scan reading, wee recorded. The fiked-gnid and biased NaJ radiological survey assessment locations were to be marked for biassed sampling for laboratory analysis by pin-flagging any location having an NaI survey reading greater than thrte times the established background or two times the established background if a background specific to the geology and topography for the area can be obtained.

Because the derived concentration guideline levels (DCGLS) for surface measurements do not apply to soil areas, direct readings are not used in the dose and risk assessinents for soil areas. Instead, soil samples were collected for dose and risk assessmment puposes. Upgrades of any LSUs were not made based on the timed NaI measurements. However, upgrades could bave been necessary based on the results of the soil sampling and analysis discussed below.

As a result of the walkover survey, five additional discretionary locations were chosen for sampling and fixed, timed messurements based on them either being recognized as a sediment collection area or due to the NaI count tate (see Fig. 6.1). Location AP-01-0I is located in a parking lot drainage ditch 
where a culvert ruming under the parking lot empties into the ditch. Location AP-01-02 is located in the woods adjacent to the parking lot near some rock outcroppings where there was a large, depressed sediment collection area Location AP-17-01 is located in a drainage area on the east side of the concrete pad. Location AP-18-01 is chosen duc to the NaI count rate [total count rate of approximately 11,000 counts per minute (cpm)] being elevated above the average background more than the other measurements. However, it was still less than twice the background of approximately $8000 \mathrm{cpm}$. Location AP-22-01 is located in a drainage area on the east side of the Hanl Road near where the area drains into a culvert that goes under Highway 58.

The results of the fixed, timed NaI measurements are shown in Table 6.18. A total of $17 \mathrm{NaI}$ measurements were riade at fixed-grid locations in LSU 1 and LSU 2. In addition, as described above, five measurements were made in LSU 1 and LSU 2 based on the walkover survey results. No NaI measurements were greater than two times a field-determined background appropriate for the geological and topographical conditions. NaI background count rates ranged fiom $5,004 \mathrm{cpm}$ to $10,578 \mathrm{cpm}$. Based on an inspection of the individual surveys, all NaI readings were less than $11,323 \mathrm{cpm}$. The maximum tissue-equivalent dose rate was $15 \mu$ rem/hour.

Results of the strveys performed in the study area and the statistical tests performed on the datz gathered in each survey unit indicate that the concrete surfaces are below the DOE surface contamination limits and within the acceptable dose-equivalent rate range for exlerior surfaces. Because all results were less than the DCGL, no statistical analysis of the data for each survey unit was required, and, therefore. the survey units can be released from a surface contamination standpoint.

Table 6.18. Fixed-timed radiological survey measurements of ED-4

\begin{tabular}{lcccc}
\hline \multicolumn{1}{c}{ Location } & $\begin{array}{c}\text { No. of } \\
\text { Measurements }\end{array}$ & $\begin{array}{c}\text { NaI } \\
\text { Min. cpm }\end{array}$ & $\begin{array}{c}\text { NaI } \\
\text { Max. cpm }\end{array}$ & $\begin{array}{c}\text { Dose equivalent } \\
\text { (nurem/b) }\end{array}$ \\
\hline LSU 1 Grid APs Locations & 8 & 6,733 & 10,140 & 0.006 to 0.009 \\
LSU 2 Grid AP Locations & 9 & 7,194 & 10,521 & 0.008 to 0.015 \\
LSU 1 Biased Sample Locations & 2 & 7,645 & 7,911 & 0.006 \\
LSU 2 Biased Sample Localions & 3 & 9,059 & 11,323 & 0.005 to 0.008 \\
\hline
\end{tabular}

Note: Sodium iodide (NaI) fixed, timed measurements are reported in counts per minute (cpm) and are not background-corrected.

AP - astessment point.

LSU -lend survey uriL

mrem/h = millirem per hour.

\subsubsection{Radiological Sanpling}

\subsubsection{Historical samples}

No historical radiological sampling data were found for this land parcel. 


\subsubsection{2007 Soil and Sediment Samples}

Soil samples were collected in the vicinity of anthropogenic features identified from historical aerial photographs and meps, and features identified during a walkover assessment of the parcel. The operation with the greatest potential to ontribute radiological contamination was transport of materials over the former railroad spur and over the existing Haul Road. The walkover assessment of the parcel identified a concrele pad and debris in the eastern tract of ED-4. Biased sampies of surface soils were collected in the vicinity of the formet warehouse and from the concrete pad. All soil samples were collected to a depth of 0 to $0.5 \mathrm{ft}$ below gtound surface (bgs). These samples (SS01. S\$O2, SSO3, and SSO4) were analyzed for baseline radionuclides. In addition, location SS01 was analyzed for transuranic (TRU) radionuclides. Sediment samples were also collected from five locations idenlified during the radiological walkover survey as sediment collection or drainage areas as discussed in Sect 6.2.1.3. The sediment samples were analyzed for baseline radionuclídes and total activity. Samples were collected in accordance with the SAP included in Appendia C. The sample iocations are discussed in the SAP.

A total of four soil and five sediment locations were sampled in the study area for radiological analysis during this effort One field duplicate was also collected at soil sample location $\$ \$ 01$ and one at sediment location 02. During the data validation process, laboratory data were assigned appropriate data validation flags. Refer to Sect. 6.1.1 for the validation flags and their definitions.

\subsubsection{2007 Groundwater Samples}

In accordance with the final SAP for Land Paret ED-4, ${ }^{\text {th }}$ groundwater samples were to be collected using temporary drive point pitzomters and existing monitoring wells. As prescribed in the SAP, five temporary piezometers were installed for the purpose of groundwater sample collection at ED-4. Section 6.1.4 describes the attempts made to collect samples, and Fig. 6.2 indicates the piezometer and weil locations that were dry and which locations were able to be sampled as part of the ED-4 investigation.

\subsubsection{Sample data analysis}

\section{Data Validation Summary}

Four surface soil sampies and five sediment samples from ED-4 were validated. During the data valjdation process, Iaboratory data wete assigned appropriate dath validation flags. Refer to Sect. 6.1.1 for the validation flagg and their deftnitions. The original analyses showed that the detection linits required to evaluate the data in comparison to the 1E-6 residential PRG screening levels were not achieved for ${ }^{90} \mathrm{Sr},{ }^{96} \mathrm{Te},{ }^{225 \mathrm{~Tb}},{ }^{233} \mathrm{U},{ }^{239} \mathrm{U}$, and ${ }^{237} \mathrm{~Np}$. Therefore, the original results were rejected and reanalyses were requested. This resulted in duplication of valid results for some of the other uranium and thotium isotopes. In these cases, the higher valid result was used for the statistical summary and risk assessment. Duplicate analyte results from gamma spectroscopy were also rejected in favor of more sensitive alpha spectroscopy results when available. Also, all fission and activation products reported from gamma spectroscopy, except for ${ }^{137} \mathrm{Cs}_{\mathrm{s}}$ were undetected and were eliminated from the final data set. In the final data set for soil samples, nine ${ }^{234} \mathrm{U}$ results were qualified as estimated due to method blank contamination, ail thorium isotopic results were qualified as estimated due to uncentainty in the chemical recovery, and the ${ }^{9 \mathrm{Sr}} \mathrm{results}$ were qualified as estimated due to duplicate results being out of limits and due to a background subtraction bias.

${ }^{15}$ BJC 2007. Sampling and Aralysis Ptan for Laud Parcel EO-A at the Eost Tennestee Technology Park, Dak Ridge, Tennesset, BJCIOR-2809, May, Dak Ridge, TN. 
For the groundwater samples, all gross beta were qualified as estimated due to the setri-quanbiative nature of the analysis that does not include volatile radionuclides (e.g., ${ }^{3} \mathrm{H}$ and ${ }^{99} \mathrm{Tc}$ ), the ${ }^{234} \mathrm{U}$ results for thre samples were qualified as estimated and undetected due to blank contamination, and the gross apha results for three samples were qualified as estimated due to blank contamination. The gross alpha/beta result for the groundwater sample collected from the temporary piezometer was greater than the sum of the alpha emitters by a factor of $8.3 / 18.5$, respectively, indicating possible missing alpha and beta emitters not analyzed for in the individual alpha and beta analyses. However, it was judged that the difference was most likely die to the natural decay chain products in the soil the sample contained undissolved solids because the samples were not filtered.

As the results for the temporary piezometer were greater than the screening values for adjusted gross alpha and gross beta, the sample was reanalyzed for gross alphafbeta, uramium tsotopes, and ${ }^{226} \mathrm{Ra}$ after filtering at the laboratory. The results of the second analysis showed ninimum uranium and ${ }^{226} \mathrm{R}$, but the gross alpha/beta results were qualifed as unusable (R) due to very high detection limits. Therefore, another analysis for gross alpha/beta was performed on a second sub-sarnple taken from the same location. Since the results were still elevated, further analyses (alpba spectroscopy, garnna spectroscopy, and total activity) were requested for qualitative purposes to belp identify the rature of the activity. The total activity results were qualified as unusable $(R)$ due to very higb detection limits.

The surb of the analytical results, including daughters assumed in equilibrium, was compared with the total activity result for each sample. The comparison showed reasonable agreement with all samples.

\section{Results}

The radiological results were intespreted in a risk evaluation (Appendix F). The surface soil sampling results are shown in Table 6.19, the sediment sampling results are shown in Table 6.20, and the groundwaler sampling results are shown in Table 6.21 by analysis type. The results are summarized along with historical data in Table 6.22 for soil samples and in Table 6.23 for sediment samples. The results for groundwater are presented in Table 6.24.

For each analysis type, the following information is listed:

- frequency of detection above background screening level,

- minimum and maximum concentrations,

- arithmetic mean,

- comparison to Zone $1 \mathrm{RL}$, and

- comparison to residential PRGs calculated for a lE-06 risk level.

The cutrent background evaluation methot is based on DOE (2003) and EPA (2002b). Backgrounds for ${ }^{252} \mathrm{Th}$ and ${ }^{236} \mathrm{Ra}$ and ${ }^{230 \mathrm{U}} \mathrm{U}$ decay chain progeny are assumed to be equal to those established for their parents due to equilibrium in nature.

Although some individual sample results for all radionuclide analytes exceeded their background values and some $\left({ }^{157} \mathrm{Cs},{ }^{49} \mathrm{Tc},{ }^{226} \mathrm{Ra},{ }^{225} \mathrm{Ra},{ }^{223} \mathrm{Th},{ }^{232} \mathrm{Th}\right.$, and ${ }^{288} \mathrm{U}$ ) exceeded their PRGs, as shown in Tables 6.22 and 6.23 for soil and sediment respectively, no individual results were greater then the Zone 1 maximum RLs and no averages were above average RLs. All survey units were found to have no residual contamination in excess of the Zone I remediation goals. 
Tabte 6.19. Radiological soil sample data from Parcel ED-4

\begin{tabular}{|c|c|c|c|c|c|c|c|c|c|c|c|c|c|c|c|c|}
\hline Sanple ID & $\begin{array}{c}\text { Sample } \\
\text { deppti (ft) }\end{array}$ & 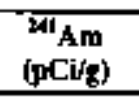 & $\begin{array}{l}{ }^{137} \mathrm{Cs} \\
(3 \mathrm{Ci} / 5) \\
\end{array}$ & $\begin{array}{l}{ }^{37} \mathrm{~Np} \\
(\mathrm{nCl} / \mathrm{g})\end{array}$ & $\begin{array}{c}\begin{array}{c}{ }^{23} \mathbf{P u} \\
(\mathrm{pCV})\end{array} \\
\end{array}$ & 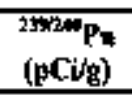 & $\begin{array}{r}226 \mathbf{R x} \\
(\mathrm{pC} i / 2) \\
\end{array}$ & $\begin{array}{l}{ }^{259} \mathrm{Ra} \\
(\mathrm{pCi} / g)\end{array}$ & 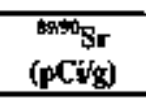 & $\begin{array}{c}{ }^{n} \mathbf{T r} \\
(\mathrm{pCr} g)\end{array}$ & $\begin{array}{c}2{ }^{28} \mathrm{Th} \\
(\mathrm{pCu} / \mathrm{g})\end{array}$ & $\begin{array}{c}{ }^{30} \mathrm{Th} \\
\left(\mathrm{pCC}^{\prime} \mathrm{g}\right)\end{array}$ & $\begin{array}{c}{ }^{2+} \mathrm{Th} \\
\left(\mathrm{pCi}^{\prime} \mathrm{g}\right)\end{array}$ & 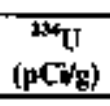 & 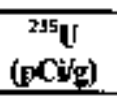 & $\begin{array}{c}{ }^{240} \mathrm{v} \\
\langle\mathrm{pCV}(\mathrm{b})\end{array}$ \\
\hline $\mathrm{N} S \mathrm{~S}-\mathrm{S} 50 \mathrm{D}$-EDA- 03 & $0-0.5$ & $-0.0825 \mathrm{U}$ & 0.356 & $0.0183 \mathrm{U}$ & $N A$ & $0.0919 \mathrm{U}$ & 0.766 & I.I & $0.00492 \mathrm{UJ}$ & $0.0715 \mathrm{U}$ & NA & $0.954 \mathrm{~J}$ & $\mathrm{NA}$ & 1.72 & 0.0964 & $1.09 \mathrm{~J}$ \\
\hline NS-550]-ED4-03 & $0 \operatorname{los}$ & -0.1230 & 0.253 & $-0.0116 \mathrm{U}$ & NA & 005140 & 0.833 & 136 & -0.0162 UH & $0.034 \mathrm{~J}$ & $123 \mathrm{~J}$ & $0.991 \mathrm{~J}$ & $1.13 \mathrm{~J}$ & 2.49 & 0.0773 & $1.07 \mathrm{~J}$ \\
\hline NS-S502-ED4-03 & $0-0.5$ & $-0.0152 \mathrm{U}$ & 0.298 & $\mathrm{Na}$ & $N A$ & NA & 0.803 & 0.913 & $0.0102 \mathrm{UI}$ & 0.02050 & $\mathrm{NA}$ & $\mathrm{NA}$ & $\mathrm{NA}$ & 1.4 & 0.0683 & $1.5 \mathrm{~J}$ \\
\hline NS-S503-EDA-03 & $0-05$ & $-0.0026 \mathrm{U}$ & 0.787 & NA & NA & NA & 1.05 & 1.01 & $0.0186 \mathrm{UJ}$ & $0.06 \mathrm{U}$ & NA & $\mathrm{NA}$ & NA & 289 & 0.142 & 3.18 \\
\hline NS-S\$N4-ED4-03 & $0-0.5$ & $0.0612 \mathrm{U}$ & 0.096 & $N_{A}$ & NA & NA & 0.688 & 112 & $-0.0972 \mathrm{Us}$ & $0.0265 \mathrm{U}$ & NA & NA & NA & 1.3 & 0.0972 & $1.45 \mathrm{~J}$ \\
\hline
\end{tabular}

NA $=$ Data not awablable

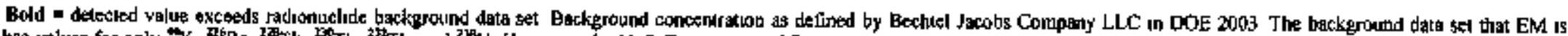

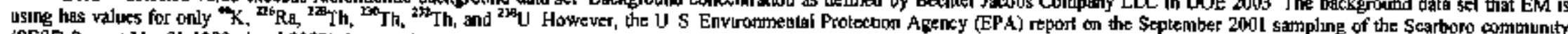

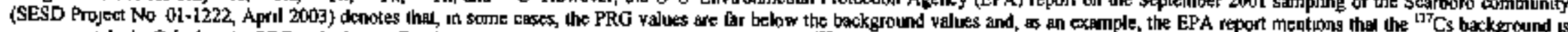

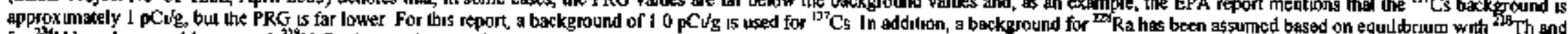

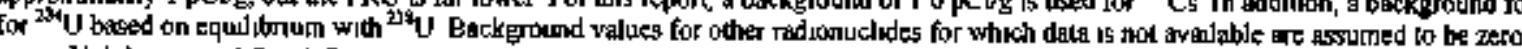

Validarton qualifier definitions

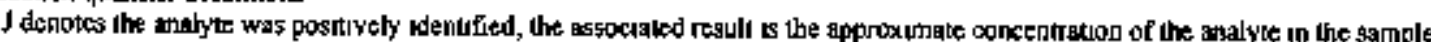

$U$ denotes the andlyte was analyzed for, but was not detected abowe the reported gample quanilitation hitut

望

Uj denotes the analyte was jot delected above the reported detection limil, which is approximated the to quality deficiency 
Table 6.20, Radiologital sediment sample data from Parcel ED-4

\begin{tabular}{|c|c|c|c|c|c|c|c|c|c|c|c|c|c|c|c|c|}
\hline Sample ID & $\begin{array}{l}\text { Semple } \\
\operatorname{depL}(\mathrm{ft})\end{array}$ & $\begin{array}{l}{ }_{2 A 1} \mathrm{Am} \\
\langle\mathrm{p} \mathrm{CH} / \mathrm{g}\rangle\end{array}$ & $\begin{array}{c}{ }^{157} \mathrm{Cs} \\
\left(\mathrm{mCi}^{\prime} \mathrm{s}\right)\end{array}$ & $\begin{array}{l}{ }^{3+} \mathrm{Np} \\
\text { (nCifg) }\end{array}$ & $\begin{array}{c}{ }^{23 n} \mathrm{Pu} \\
(\mathrm{pCug})\end{array}$ & $\begin{array}{l}2392 \times 10 \mathrm{Pu} \\
([\times C / g)\end{array}$ & $\begin{array}{c}{ }^{276} \mathrm{Re} \\
(\mathrm{NeV})\end{array}$ & 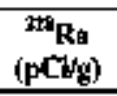 & 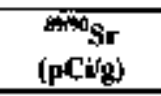 & $\begin{array}{c}{ }^{*} \mathrm{Tc} \\
(\mathrm{pCHg})\end{array}$ & $\begin{array}{l}{ }^{221} \mathrm{Th} \\
\left(\mathrm{pCi}^{\prime} / \mathrm{g}\right)\end{array}$ & 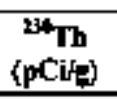 & $\begin{array}{c}{ }^{2{ }^{2}} \mathrm{Th} \\
(\mathrm{pCl} / \mathrm{y})\end{array}$ & $\begin{array}{c}{ }^{24}[\mathrm{G} \\
\left(\mathrm{p} C \mathrm{~V}_{\mathrm{g}}\right)\end{array}$ & $\begin{array}{c}(\mathrm{nax} v \\
(\mathrm{pCi} f \mathrm{~g})\end{array}$ & $\begin{array}{c}{ }^{130} U \\
\left(p C W_{g}\right)\end{array}$ \\
\hline NS-01-SD-ED4-03 & $0-0.5$ & $0.193 \mathrm{U}$ & 0.158 & $\mathrm{NA}$ & $\mathrm{NA}$ & $N A$ & 1.47 & 1. 12 & $-4.00235 \mathrm{UJ}$ & $0.0305 \mathrm{U}$ & $\mathbf{N A}$ & NA & $N A$ & $1.39 \mathrm{~J}$ & 0.125 & 1.4 \\
\hline NS-12-DI-ED4-03 & $0-0.5$ & $0.0799 \mathrm{U}$ & 0.12 & $\mathrm{NA}$ & NA & NA & 1.07 & {$[.47$} & -0.00549 (JJ & 0.0403 & NA & $\mathrm{NA}$ & NA & 199 & 0.144 & 1.48 \\
\hline NS-02-SD-ED4-03 & $0-0.5$ & $0.162 \mathrm{v}$ & 0.78 & NA & NA & $\mathrm{NA}$ & 1.06 & 0.39 & -0.00819 U४ & $0.0262 \mathrm{U}$ & NA & NA & NA & 1.99) & 0.0897 & 1.48 \\
\hline NS- $-13-S D-E D 4-03$ & $0-0.5$ & $0.211 \mathrm{U}$ & 0.416 & NA & NA & $\mathrm{NA}$ & 0.701 & 0.935 & $-0.00969 \mathrm{UJ}$ & 0387 & NA & $\mathrm{NA}$ & $\mathrm{NA}$ & 2.41 & 0.165 & $1.92 \mathrm{~J}$ \\
\hline NS- $-14-S D-E O 4-03$ & $0-0.5$ & 0.10010 & 0.0961 & NA & NA & NA & 1.05 & Is & -0.020865 & 0.0388 & $\mathrm{NA}$ & $\mathrm{NA}$ & NA & $1.1 \mathrm{~J}$ & 0.0944 & $1.36 \mathrm{~J}$ \\
\hline NS-OS-SD-ED4-03 & $0-0.5$ & $0.0971 \mathrm{U}$ & 0.149 & $\mathrm{NA}$ & NA & $\mathrm{NA}$ & 1.07 & 1.85 & $0.00436 \mathrm{U}$ & $0.0190 \mathrm{U}$ & NA & $\mathrm{NA}$ & NA & 1.68 & $0.13 \mathrm{I}$ & $1.98,7$ \\
\hline
\end{tabular}

NA = Dala not draileble.

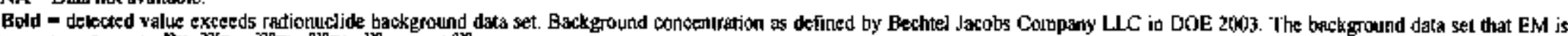

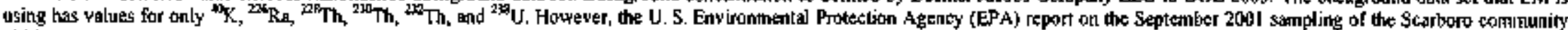

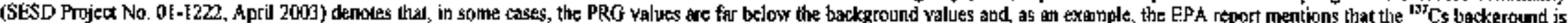

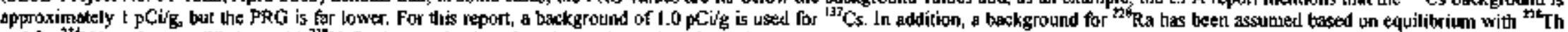

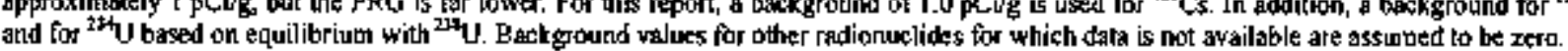

Validation qualificer definitions:

J denotes the analyle was positively identified; the associaled resalt is the approximate concentration of the malyte in the sample

U denoles the andyle wes analyzad lor, but was not delected above the reparted sample quantitation liusic

UJ denous the analyte was not detected above the reported detection limit, which is approximated due to guality deficisncy. 
Table 6.21. Radłological groundwater sampte data from Parcel ED-4

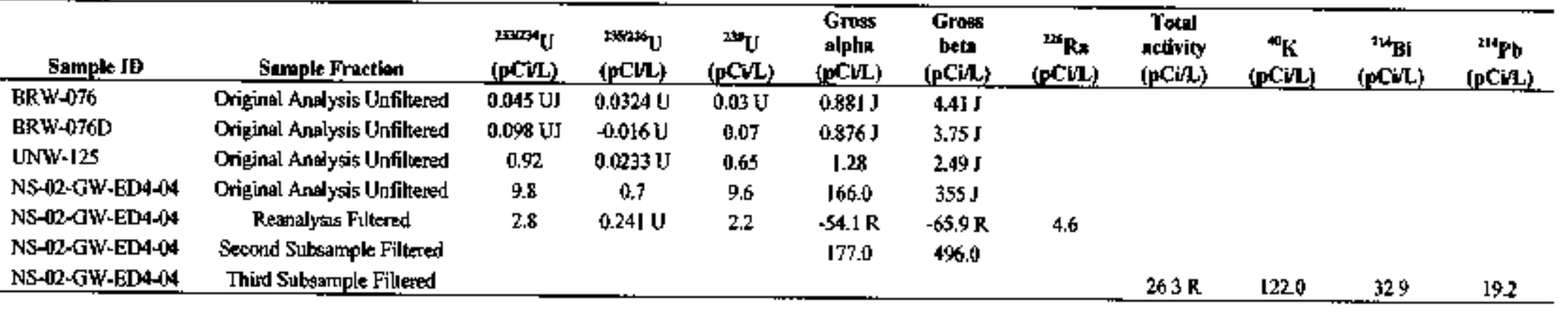

Valudation qualıfier definitions

J denotes the analyte was positively wend ficed, the associated resull is the approxirnate concentration of the analyte in the sample

U denrates the analyte was aralyzed fo, but was not deteded abowe the seported sample quantiation licoit

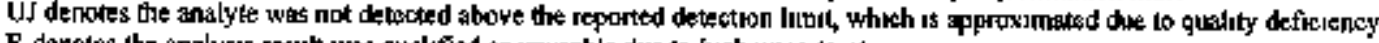

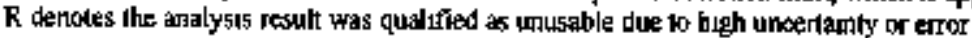




\begin{tabular}{|c|c|c|c|c|c|c|c|c|c|c|c|c|}
\hline Analyte & $\begin{array}{c}\text { Frequency } \\
\text { of } \\
\text { tetections }\end{array}$ & 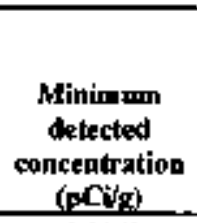 & $\begin{array}{c}\text { Maximum } \\
\text { deseted } \\
\text { concentration } \\
\left(p C^{\prime} / q\right)\end{array}$ & $\begin{array}{c}\text { Aversist } \\
\text { detected } \\
\text { concendration } \\
\text { (pCig) }\end{array}$ & $\begin{array}{l}\text { Backigrownd } \\
\text { reference } \\
\text { level" } \\
\text { (pCi/s) }\end{array}$ & $\begin{array}{l}\text { Frequency } \\
\text { of } \\
\text { detections } \\
\text { exceeding } \\
\text { backgriound } \\
\text { reference }\end{array}$ & $\begin{array}{c}\text { Marimum } \\
\text { RL }\end{array}$ & $\begin{array}{l}\text { Frequency } \\
\text { af detexts } \\
\text { extexding } \\
\text { maximain } \\
\text { RL } \\
\end{array}$ & $\begin{array}{l}\text { Average } \\
\text { Zope } 1 \\
\text { remediation } \\
\text { leves } \\
\text { (pCi/s) }\end{array}$ & $\begin{array}{c}\text { Exceed } \\
\text { Zone I } \\
\text { gverage } \\
\text { reneediation } \\
\text { Leyel? }\end{array}$ & 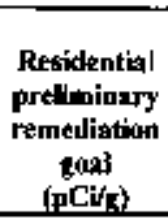 & $\begin{array}{c}\text { Prequency } \\
\text { of detests } \\
\text { expeeding } \\
\text { prelimimary } \\
\text { remediation } \\
\text { goal }\end{array}$ \\
\hline Americium-24I & 0,4 & -0.0152 & 0.0612 & 0.02 & 0 & $0 / 4$ & & NA & & $\mathrm{N} \mathrm{A}$ & 1.87 & $0 / 4$ \\
\hline Cesium-137 & $4 / 4$ & 0.096 & 0.787 & 0.38 & I & $0 / 4$ & 20 & 64 & 2 & No & 0.0597 & $4 / 4$ \\
\hline Neptunium-237 & $0 / 1$ & $\mathrm{ND}$ & ND & ND & 0 & ort & 50 & $b^{\prime \prime}$ & 5 & No & 0.13 & 01 \\
\hline Plutonium-239 & $\theta / 1$ & ND & ND & $\mathrm{ND}$ & 0 & orl & & $\mathrm{NA}$ & & NA & 2.6 & $\mathrm{H}_{1}$ \\
\hline Radiurn-226 & $4 / 4$ & 0.6888 & 1.05 & 0.84 & 1.25 & $0 / 4$ & 15 & $0 / 4$ & 5 & No & 0.0124 & $4 / 4^{\circ}$ \\
\hline Radilizum-228 & $4 / 4$ & 0.913 & 1.36 & i. 1 & 1.95 & $0^{\prime} 4$ & & NA & & NA & 0.67681899 & $4 / 4^{b}$ \\
\hline Strontium-90 & $1 / 4$ & $0.0186 \mathrm{~J}$ & $0.0186 \mathrm{~J}$ & 0.02 & 0 & $1 / 4$ & & $\mathrm{NA}$ & & NA & 0.231 & $0 / 4$ \\
\hline Technetium-99 & $2 / 4$ & 0.86 & 0.0715 & 0.07 & 0 & $2 / 4$ & & $N A$ & & $\mathrm{NA}$ & 0.25 & $0 / 4$ \\
\hline Thorlume-228 & III & $1.23 \mathrm{~J}$ & $\mathrm{E} .23 \mathrm{~J}$ & 123 & 1.86 & $0 / 1$ & & $\mathrm{NA}$ & & $\mathrm{NA}$ & $0.0768 \mathrm{1} 899$ & $1 / t^{b}$ \\
\hline Thoriu|a-230 & $1 / 1$ & 0.991 & $0.991 \mathrm{~J}$ & 0.99 & 1.2 & $0 / 1$ & & NA & & $\mathrm{NA}$ & 3.5 & $0 / 1$ \\
\hline Thoriumin-232 & $1 / 1$ & $1,12 \mathrm{I}$ & $2.12 \mathrm{~J}$ & 1.32 & 1.95 & $0 / 1$ & 15 & $0 / 1$ & 5 & $\mathrm{No}$ & 0.07681899 & $1 / I^{\phi}$ \\
\hline Uranium-234 & $4 / 4$ & $0.939 \mathrm{~J}$ & 2.12 & 0.31 & 1,47 & $1 / 4$ & 7,000 & $0 / 4$ & 700 & No & 4.02 & $0 / 4$ \\
\hline Uraniuun-235 & $4 / 4$ & 0.0683 & 0.142 & 0.1 & 0 & $4 / 4$ & 80 & $0 / 4$ & 8 & No & 0.195 & $0 / 4$ \\
\hline Uranium-238 & $4 / 4$ & 1.95 & 2.89 & 2.38 & 1.47 & $4 / 4$ & 500 & $0 / 4$ & 50 & No & 0.743 & $4 / 4$ \\
\hline
\end{tabular}

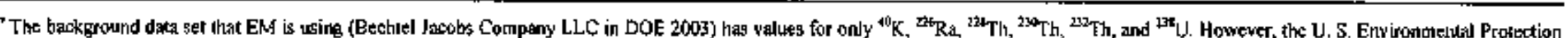

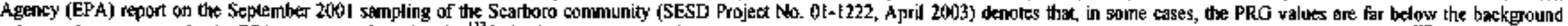

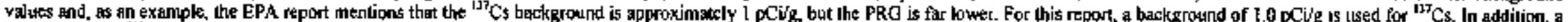

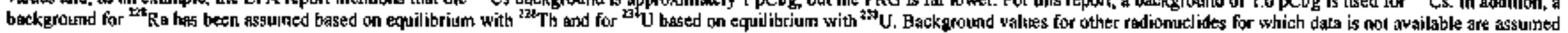
to be zero.

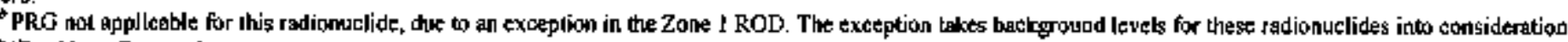

$\mathrm{ND}=$ None Delected

NA = Not applicable, not audilable or insufficent data ka calculate the statistic.

pCily - picocirajes per gram.

RL - remediation lewel. 
Table 6.23. Parcel ED-4 saumary statistics for radionuetides in sodiment

\begin{tabular}{|c|c|c|c|c|c|c|c|c|c|c|c|c|}
\hline Anatyte & $\begin{array}{c}\begin{array}{c}\text { Prequency } \\
\text { of } \\
\text { detections }\end{array} \\
\end{array}$ & $\begin{array}{l}\text { Mhininm } \\
\text { detected } \\
\text { comcentration } \\
\text { (pCifr) }\end{array}$ & $\begin{array}{c}\text { Maximm } \\
\text { detected } \\
\text { concentrstion } \\
\text { (pCifg) }\end{array}$ & $\begin{array}{l}\text { Avernge } \\
\text { detested } \\
\text { concentration } \\
\text { (ncivg) }\end{array}$ & $\begin{array}{c}\text { Backgraund } \\
\text { retereuce } \\
\text { bevel } \\
\text { (pCis) } \\
\end{array}$ & $\begin{array}{l}\text { Frequency } \\
\text { of } \\
\text { detections } \\
\text { exceeding } \\
\text { backgronnd } \\
\text { reterenee }\end{array}$ & $\begin{array}{c}\text { Maximnm } \\
\text { RI. } \\
\end{array}$ & $\begin{array}{c}\text { Freqneacy } \\
\text { of detects } \\
\text { excetding } \\
\text { maximinis } \\
\text { RL }\end{array}$ & 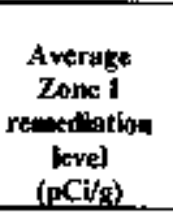 & $\begin{array}{l}\text { Exceed } \\
\text { Zone } 1 \\
\text { axerape } \\
\text { remedlotion } \\
\text { level? }\end{array}$ & 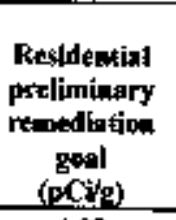 & $\begin{array}{l}\text { Frequency } \\
\text { of deteets } \\
\text { exceeding } \\
\text { preliminoty } \\
\text { remediation } \\
\text { gapal } \\
\end{array}$ \\
\hline Americium 241 & $5 / 5$ & 0.0971 & 0.211 & 0.15 & 0 & $5 / 5$ & & $\mathrm{NA}$ & & NA & 1.87 & a/s \\
\hline Cessium-137 & $5 / 5$ & 0.0961 & 0.416 & 0.2 & 1 & ors & 20 & $0 / 5$ & 2 & No & 0.0597 & $5 / 5$ \\
\hline Radium-226 & $5 / 5$ & $0.70 \mathrm{t}$ & 1,47 & 1.07 & 1.25 & $1 / 5$ & IS & ats & 5 & $\mathrm{No}$ & 0.0124 & $5 / 5^{*}$ \\
\hline Ratium-228 & $\$ 15$ & 0.935 & I.85 & 1.38 & 1.95 & $0 / 5$ & & NA & & $N A$ & 0.07681899 & $s / S^{*}$ \\
\hline Strantium-90 & $0 / 5$ & ND & ND & ND & 0 & $0 / s$ & & NA & & NA & 0.231 & $0 / 5$ \\
\hline Techbetitu-99 & $3 / 5$ & 0.0388 & 0.387 & 0.16 & 0 & $3 / 5$ & & NA & & NA & 025 & $1 / 5$ \\
\hline Uran'́n, & $5 / 5$ & L.IJ & 1.81 & 1.45 & 1.47 & $2 / 5$ & 1,000 & $0, s$ & 700 & $N$ & $4.02 \mathrm{E}+100$ & $0 / 5$ \\
\hline Unanium-235 & $5 / 5$ & 0.0944 & 0.165 & 0.13 & 0 & $5 / 5$ & 80 & ats & 8 & No & 0.195 & 065 \\
\hline Uranium-239 & $5 / 5$ & I.4 & 3.08 & 1.94 & 1.47 & $4 / 5$ & 500 & 0,5 & 50 & No & 0.743 & $5 / 5$ \\
\hline
\end{tabular}

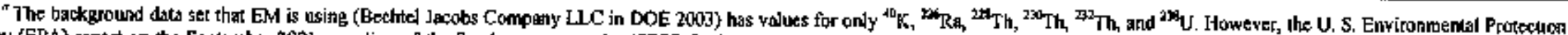
Afency (EPA) report on the Seplember 200] sampling of the Scarturo community (SESD Project No. 0J-J222, Aprit 2003) denotes that, in some cases, the PRG values are far below the background

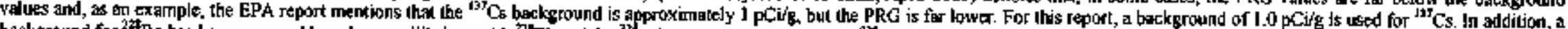

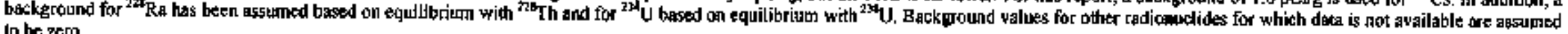
to be zero.

PRG ort epplicable for this radionuclide, due wo an exception in the Zone I ROD. The exception takes background levels for these cadionuelides into consideration. $\mathrm{ND}=\mathrm{N}_{\mathrm{N}} \mathrm{Ne}$ Detected

NA = Not applicable, not arajlable or ifsufficient dita to calculate the statistic.

PCíg = picocuries per gram.

RL - remedialion level. 
Table 6.24. Radlonuclides sumenary for groundwater from Pareel ED-4

\begin{tabular}{|c|c|c|c|c|c|c|}
\hline Anolyte & $\begin{array}{c}\text { Frequency of } \\
\text { detections }\end{array}$ & $\begin{array}{l}\text { Mlutmam } \\
\text { detected } \\
\text { crncentration } \\
(\mathrm{pCi} / \mathrm{L})\end{array}$ & $\begin{array}{l}\text { Maximum } \\
\text { detected } \\
\text { concentration } \\
\text { (pCi/L) }\end{array}$ & $\begin{array}{c}\text { Average } \\
\text { deterted } \\
\text { concentration } \\
\text { (pClL) }\end{array}$ & $\begin{array}{c}\text { Drinking water } \\
\text { screening level } \\
(\mathrm{pCt} / \mathrm{L})\end{array}$ & $\begin{array}{c}\text { Frequency } \\
\text { of detects } \\
\text { erceeding } \\
\text { drinking water } \\
\text { screening level }\end{array}$ \\
\hline 231204 & $2 / 3$ & 0.92 & 2.8 & 1.9 & 30 & $0 / 3$ \\
\hline${ }^{25 \sin 36} \mathrm{U}$ & $1 / 3$ & 0.7 & 0.7 & 0.7 & 30 & $a / 3$ \\
\hline${ }^{23} \mathrm{~B}_{\mathrm{U}} \mathrm{J}$ & $2 / 3$ & 0.07 & 2.2 & 1.1 & 30 & ars \\
\hline Gross Alpha & $3 / 3$ & .88 & 177 & 59.7 & 15 & $1 / 3$ \\
\hline Gross Beta & $3 / 3$ & 2.5 & 4.96 & 167 & 50 & $1 / 3$ \\
\hline${ }^{70} \mathrm{Ra}$ & $1 / 1$ & 4.6 & 4.6 & 4.6 & 20 & 03 \\
\hline
\end{tabular}

RL = remodistion leyel 
For each groundwater andysis type, the following information is listed:

- frequency of detection,

- aritbmetic mean, and

- comparison to EPA drinking water screening values.

The results for the groundwater sample collected from the newly installed piezometer NS-02-GWED4 showed high gross alpha and gross beta results. The high result coutd not be accounted for by the uranium and radium measured in the sample. The alpha spectra did not confirm the presence of significant alpha activity in the sample. The gamma spectra provided some clues and showed that naturally occurring ${ }^{49} \mathrm{~K}$ could account for over $100 \mathrm{pCV} / \mathrm{L}$ of beta activity. Also, ${ }^{214} \mathrm{Bi}$ and ${ }^{214} \mathrm{~Pb}$ were detected at levels close to $20 \mathrm{pCi} / \mathrm{L}$. These are ${ }^{226} \mathrm{Ra}$ daughters and would indicate the presenee of the entìe ${ }^{276} \mathrm{Ra}$ decay chain since all ${ }^{225} \mathrm{Ra}$ daughters have a relatively short half-life. That could account for five or six times the activity of the measured ${ }^{214} \mathrm{Bi}$ or ${ }^{214} \mathrm{~Pb}(20$ to $30 \mathrm{pCi} / \mathrm{L})$ for the gross alphe activity and the gross beta activity. The fact that ${ }^{226} \mathrm{Ra}$ was not detected might be explained by the fact that the ${ }^{272} \mathrm{Rn}$ daughter is a gas and could have been absorbed in the liquid phase of the sample if ${ }^{226} \mathrm{Ra}$ is in the solids that were filtered away. It is also possible that ${ }^{222} \mathrm{R}$ could have becn drawn into the liqguid sample from the air during filtering since vacum suction was used. The measured values do not seem to be consistent between the different analyses. This may be due to variations in how the sample is preserved and bandled, which can influence bow much activity in the solids is dissolved or in a colloidal form that would pass through the filter.

EPA has suggested that the data collected by the state of Tennessee for the Happy Valley Spring in ED -3 may be used to evaluate the groundwater for this area of ED-4. The radiological data from that data set are shown in Table 6.25. While the gross alpha and gross beta results are consistently less than $10 \mathrm{pCi} / \mathrm{L}$, results for ${ }^{314} \mathrm{Bi}$ and ${ }^{214} \mathrm{~Pb}$ are consistently around 60 to $70 \mathrm{pCi} / \mathrm{L}$ for each and with one sample showing approximately $200 \mathrm{pCl} / \mathrm{L}$ for each. From the evaluation of all data, it is apparent that the elevatod alpha and beta activity is likely due to naturally occuring radionuclides in the solids or absotbed into the liquid and is not from process-related radionuclides. Although ${ }^{214} \mathrm{Bi}$ and ${ }^{214} \mathrm{~Pb}$ are ${ }^{238} \mathrm{U}$ decay chein daughters, they are not process related due to the long half-Iife internediate isotopes in the decay chain berween the chemically separated process uranium and thcse daughters. 
Table 6.25. Ratiological spring tample data from Happy Valley collected by the state of Tennessee

\begin{tabular}{ccccc}
\hline Date & $\begin{array}{c}\text { Gross } \\
\text { Alphis } \\
\text { (pCiL) }\end{array}$ & $\begin{array}{c}\text { Gross } \\
\text { Beta } \\
(\mathrm{pCi})\end{array}$ & $\begin{array}{c}{ }^{314} \mathbf{B i} \\
(\mathrm{pCi})\end{array}$ & $\begin{array}{c}{ }^{214} \mathrm{~Pb} \\
(\mathrm{pCi})\end{array}$ \\
\hline $1 / 13 / 97$ & 0.7 & 2.3 & 203 & 198 \\
$11 / 2 / 98$ & 1.6 & 5.7 & 20 & $\mathrm{ND}$ \\
$5 / 25 / 99$ & -0.4 & 3.3 & 67 & 60 \\
$11 / 15 / 99$ & -0.5 & 1.9 & 35 & 25 \\
$3 / 1400$ & 0.5 & 6 & 62 & 54 \\
$10 / 23 / 00$ & 2.8 & 10.2 & 73 & 68 \\
$2 / 7 / 01$ & 0.9 & 3.6 & 42 & 24 \\
$3 / 25 / 02$ & 0.8 & 3.8 & $\mathrm{ND}$ & $\mathrm{ND}$ \\
$10 / 16 / 02$ & -0.06 & 1.4 & 56 & 71 \\
\hline
\end{tabular}

Validation qualifer definitions:

J denotes the analyte was positively identified; the associated result is the approximate concentration of the amalyte in the sample.

U denotes the analyte was analyzed for, but was not detected above the reported sample quantitation limit.

UI denotes the auslyte was not detected above the reported delection limit, which is approximated due to quality deficiency.

$R$ denotes the analysis result was qualified as unusable tue $w$ high uncertainty or error.

ND denotes not detected. 


\section{REFERENCES}

Aero (Aero Services Corporation) 1942. Untitled topographical map of Oak Ridge Reservation showing Pine Ridge and Bear Creek Valley, Sheet B-I, RQ 10898, prepared by Aero Services Corporation for Stone and Webster, November 2, 1942.

BJC (Bechtel Jacobs Company LLC) 1996. Radiation Protection Program, BJC-SH-04, Vol. 1, Sect. SHB-4012, "Radiation Contamination Control and Monitoring," Oak Ridge, TN.

BJC 2000s. Destgn of Radiological Surveys of Potential Lease Space at East Tennessee Technology Park, Oak Ridge, Tennessee, BJClOR-554, Oak Ridge, TN, March.

BJC 2002a Surveillance and Maintenance Plan for Remedial Action and Past Cleanup O\&M Sites at the East Tennessee Technology Park, Oak Ridge, Tennersee, K/ER-54/R9, December.

BJC 2002b. Design of Radiological Swney and Sampling to Support Title Transfer or Lease of Property on the Department of Energy Oak Ridge Resenation, BJC/OR-554-R I, Oak Ridge, TN, September.

BJC 2007. Walkover Assessment Instruction for Heritage Center Propenty Transfers at the East Tennessee Technology Park, Oak Ridge, Tennessee, BJCJOR-2808, Oak Ridge, TN, May.

C\&CCC (Carbide and Carbon Chemical Comoration) 1946a. K-25 \& K-27 Plot Plan, Drawing No. AW-1M, dated December 21, 1945 (Rev. 3, dated Decernber 26, 1946), Oak Ridge K-25 Site, Oak Ridge, TN.

C\&CCC 1946b. Reference Plot Plom, Drawing No. ERS-1, dated January 24, 1946, Engineering Records Section.

C\&CCC 1947. K-25 \& K-27 Plot Plan, Drawing No. AW-10M (supersedes AW-1M), dated January 31, 1947, Carbide and Carbon Chemicals Corporation, Dak R.jdge K-25 Site, Oak Ridge, TN.

C\&CCC 1951a. K-25 \& K-27 Plot Plan, Drawing No. AW-10M (Rev. 8, dated August 17, 1951), Carbide and Carbon Chemicals Corporation, Oak Ridge K-25 Site, Oak Ridige, TN.

C\&OCC 1951b. "Figure 1: Location Map Underground Transmission Lines - K-704 to Process Area" ( $K-25$ \& K-27 Plot Plan, Drawing No. AW-10M, Rev. 8, August 17, 1951, modified into figure that shows underground transmission lines and K-704 \$witchhoust).

C\&CCC 1955. Area Plot Plan, Drawing No. S-KT-K100 (supersedts AW-10M), dated lune 24, 1953 (Rev. 2, dated June 22, 1955), Carbide and Carbon Chernicats Company, Division of Union Carbide and Carbon Corporation, K-25 Plant, Oak Ridge, TN.

Catalytic, Inc. 1971, K-1037 "Area Plan," Drawing. No. E-101, Rev. 0, dated May 20, 1971, prepared for Union Carbide Comporation-Nuclear Division, Oak Ridge Gascous Diffusion Plant, by Catalytic, Inc., Philadelphia, PA.

CDM (CDM Federal) 2005. Email communication regrarding underground storage tanks from S. T. Goodpasture of CDM Federal, Oak Ridge, TN, on March 11. 
CFR (Code of Federal Regulations) 1997a. Rentification and Listing of Hazardots Waste, 40 CFR 261, Appendix VHI, "Hazardous Constituents."

CFR 1997b. Designation, Reportable Quontities, and Notiflcation, 40 CFR 302.4, Table 302.4, "List of Hazardous Substances ard Reportable Quantities."

CFR 1999. Occupational Radiation Protection, 10 CFR 835, Appendix D, "Surface Radioactivity Values."

COE (U. S. Amy Corps of Enginers) 1998. Personal communication with Bil Banes of the U. S. Anny Corps of Engineers on April 6, 1998.

DOE (U. S. Department of Energy) 1940s-Present. ED-4 Site Photographs, DOE Photo Archives, Oak Ridge, TN.

DOE 1990a. Radiation Protection of the Public and the Environment, DOE Order 5400.5, Fig. IV-1, "Surface Contamination Guidelines," p. IV-6, Washington, D.C., Ftbriary.

DOE 1990b. Radiation Protection of the Public and the Environment, DOE Order $\$ 400.5$, Sect. IV.4,C, Washíngton, D.C.

DOE 1992. Federal Facility Agrement for the' Oat Ridge Reservatton, DOE/OR-1014, U. S. Environmental Protection Agency Region 4, U. S. Department of Energy, and Tennessee Department of Environment and Conservation, Washington, D.C.

DOE 1996. Groundwater Remedial Site Evaluation Report for the Oak Ridge $K-25$ Site, DOE/OR/01$1468 \& \mathrm{D} 1$, Office of Environmental Maragement, Oak Ridge, TN.

DOE I997a Evaluation of McKinney Ridge Study Area, Environmental Restoration Footprint Rechuction Process, DOE/OR/01-1511\&D1/R1, Office of Environmental Management, Oak Ridge, TN.

DOE 1997 b. Remedial Site Evahtation Report for the Happy Valley Campsite at the Oak Ridge Reservation, Oak Ridge, Tennessee, DOE/OR/02-1654\&D1, Office of Envinonmental Management, Oak Ridge, TN.

DOE 1999. "Memorandum: Information Brief on Long-Term Control of Property," October 26, 1999, from Andrew Wallo III, Director, Air, Water and Radiation Division, Office of Environmental Policy and Assistance, to Distribution (transmits EH-412-0014/1099, The Long-Term Control of Property: Overview of Requirements in Orders DOE 5400. I \& DOE 5400.5).

DOE 2000. Independent Investigation of the East Temessee Technology Part, Volume I: Past Environment, Safety, and Health Practices, ETTP-363, p. 18. U. \$. Departnent of Energy, Office of Oversight, Environment, Safety and Health, Washington, D.C., October.

DOE 2002. Recond of Decision for Interim Actions in Zone 1, East Tennessee Technology Park, Oak Ridge, Tennessee, DOE OR/01-1997\&D2, U. S. Department of Energy, Office of Environmental Management, October.

DOE 2003. Soil Background Supplemental Data Set for the East Tennessee Technology Park, Oak Ridge, Tennessee, DOE/OR/01-2105\&D1, U.S. Department of Encrgy, Office of Environmental Management, September. 
DOE 2005. Remedial Action Work Plan for Dynamic Verification Strategy for Zone I East Tennessee Technology Park, Oak Ridge. Tennessee, DOE/OR-01-2182\&D2, U. S. Departnent of Energy, Office of Environmental Management, Oak Ridge, TN, February,

DOE 2006a, Phased Construction Completion Report for the $K-1007$ Ponds Area and Powerhotse Area in Zone $l$ at East Tennessee Technology Park, Oak Ridge, Tennessee, DOE/OR/01-2294\&D2, U. S. Department of Energy, Office of Environmental Management, Oak Ridge, TN.

DOE 2006b. Fiscal Year 2006 Phased Construction Completion Report for the Zone 2 Soils, Slabs, and Subsurface Strichures at East Tennessee Technology Park, Oak Ridge, Tennessee, DOE/OR/0123I7\&DI, U. S. Department of Energy, Office of Environmental Management, Oak Ridge, TN.

DOE 2007a. FY 2008 Phased Construction Completion Report for the Front Lawn in Zone 1 at East Tennessee Technology Park, Oak Ridge, Tennessee, DOE/OR/01-2367\&D0, U. S. Department of Energy, Office of Environmental Management, Oak Ridge, TN.

DOE 2007b. Final \$itewide Remedial Investigation and Feasibility Study for the East Tennessee Technology Park, Oak Ridge, Tennessee, DOE/OR01-2279\&D3, U. S. Departnent of Energy, Office of Environmental Maragement, Oak Ridge, TN.

Energy Systems (Lockheed Martin Energy Systems, Inc.) 1995. Site Descriptions of Environmental Restoration Units at the Oak Ridge K-25 Site, Oak Ridge, Tennessee, K/ER-47/R1, Oak Ridge, TN.

Energy Systems 1996a. Real Estate Section of Comprehensive Environmental Response, Compensation, and Liability ACt (CERCLA) Sect. 120(h) Review, authored by W. W. Teer, Jr., Real Estate Manager, Lockheed Martín Energy Systems, Inc., Oak Ridge, TN, August 9.

Energy Systems 1996b. Oak Ridge Outdoor Radiological Characterization Phase II, K/HS-620, Lockheed Martin Energy Systems, Inc, Oak Ridge, TN.

Energy Systems 1997. Oak Ridge Reservation Anmal Site Environmental Report for 1997. ES/ESH-78, Lockheed Martin Energy Systems, Inc., Oak Ridge, TN.

Energy Systems (Martin Marietta Energy Systems, Inc) 1992. The Oak Ridge K-2S Site Technical Site Information, K/EN/SFP-3, Oak Ridge, TN, July.

EPA (U.S. Envionmental Protection Agency) 1989. Risk Assessment Guidance for Superfund, Vol. 1. Ftuman Healh Evaluation Manual (Part A), Interim Final, EPA/540/1-89/002, Office of Emergency and Remedial Response, Washington, D.C., December.

EPA 1999. National Functional Guidelines for Organic Data Review, EPA-5401R-99-008, Washington, D.C.

EPA 2002b. Guidance for Comparing Background and Chemical Concentrations in Soil for CERCLA Sites, EPA 540-R-01-003, U. S. Environmental Protection Agency, Washington, D.C.

EPA 2004. National Functional Guidelines for Inorganic Data Review, EPA 540-R-04-004, Washington, D.C.

Giles, G., and J. Story 1997. "The Low-Down on Low Flow", Site Remediation News 9(3), Bureau of Ground Water Pollution Abatement, New Jersey Department of Environmental Protection. 
Goddard, P. L., A. J. Legeay, D. S. Pesce, and A. M. Stanley 1995. Site Descriptions of Envinonmental Restoration Units at the Oat Ridge K-25 Site, Oat Ridge, Tennessee, K/ER-47/R1, Lockheed Martin Energy Systerns, Ine., Oak Ridge K-25 \$ite, Oak Ridge, TN, November.

Jones (J. A. Jones Construction Cornpany, Inc.) 1944. "General Layout, K-25 Area, Sanitary Water Distribution," Drawing No. 20711, Clinton Engineer Works, Oak Ridge, TN.

Kellex (Kellex Corporation) 1946. K-25 and K-27 Plot Plan \& Status of Construction, Drawing No. FD-01-AA-02, dated February 27, 1944 (Rev. 9, dated March 31, 1946).

Kellex (Undated), Contour Map, Process \& Power Plant Area, Drawing No. SK-T405-M-01-A10.

Legery, A. J., Goddard, P. L., Stanley, A M., and Pesce, D. S. (unpublished). Hazardous Waste Sites Historical Investigation, 1987-1993, Oak Ridge K-25 Site, Oak Ridge, TN.

Lemiszki, P. J. 1994. Geological Mapping of the Oat Ridge K-25 Site, Oak Ridge, Tennessee, KVR-111, Environmental Restoration Division, Oak Ridge K-25 Site, Martin Marietta Energy Systerns, Inc., Oak Ridge, TN.

Mitchell, M E, 1983a, Environmental, Health, and Safety Concerns for the New ORGDP Contractor, K/HS-5 (Rev.), Health, Safety, and Environmental Aftairs Division, Union Carbide Corporation, Nuclear Division, Oak Riđge Gaseous Diffusion Plant, Oak Ridge. TN, May.

Mitchell, M. E. 1983b. Environmental, Health, and Safety Concerns for the New ORGDP Contractor, K/HS-S (Rev, 2), Health, Safety, and Environmental Affairs Division, Union Carbide Corporation, Nuelear Division, Oak Ridge Gaseous Diffusion Plant, Oak Ridge, TN, July.

NRC (Nuclear Regulatory Comunission) 1997a. Multi-Agency Radiation Survey and Site Investigation Manual (MARSSM), Find Edition, NUREG-1575, Washington, D.C., December.

NRC 1997b. A Proposed Nonparametrical Statistical Methodology for the Design and Analysis of Find Status Decommissioning Sturveys, Final Edition, NUREG-1505, Washington, D.C., Deeember.

NRC 1997c. Minimum Detectable Concentrations with Typical Radiation Suney Instruments for Varlous Contaminants and Field Conditions, Table 5.1, NUREG-1507, Washington, D.C., December.

ORPS (Occurrence Reporting and Processing System) 2003. Oceurrence Reporting and Processing System database.

Prince, R. P. 2004a. Data Extracted from Secret-Restricted Dato Documents KZ-7976-4, KZ-7976-5, K2-7976-6, and From Confidential-Restricted Data Dacument. KZ-7976-I Cascade Log Books (U) - As Relating to Historic Materials Releases at Selected Facilities, ETTP-696, Bechtel Jacobs Company LLC, Oak Ridge, TN.

Prince, R P. 2004b. Data Extracted from Secret-Restricted Data Documents K-SS-46, Parts 1, 2, \& 4. KSS-56, Part 3, K-SS-70, Fart 3, K/P-6749, Parts 5, 6. \& 7, K/SS-439, K/SS-444, K/SS-451, Par 1 . Shift Superintendent's Log Books (U)-As Retating to Historic Materials Releases at Selected Facilities, ETTP-697, Bechtel Jacobs Company LLC, Oak Ridge, TN. 
Prince, R. P. 2004c. Data Extracted from Secret-Restricted Data Document K-SS-451, Part 2, Shift Superintendent's Log Book (U) - Regarding Events During the December I985 Release of ${ }^{\circ 0} \mathrm{Sr}$ Through White Oak Dam, ETTP-698, Bechtel Jacobs Company LLC, Oak Ridge, TN.

Prince, R. P. 2004d. Data Extracted from Secret-Restricted Data Documents K-Z-7975, Parts 2,4, \& 8, KC-377. Parts 1 \& 2, KP-1165, Parts 1, 2, \& 8, KP-1666, Parts 2, 4, and 8. Shift Stiperintendent's Log Book $(U)$ - Regarding Materiat Reteases as Observed from the Former Oak Ridge Gaseous Diffision Plant, ETTP-699, Bechtel Jacobs Company LLC, Oak Ridge, TN.

Prince, R. P. 2004e. Data Extracted from K-25 Shift Superintendent's Daily Logs for: January 1986 thru Febrwary 1986. September 1986 thru October 1986, and November 1986 thrw December 1986 Regarding Material Relecses as Observed from the Former Oak Ridge Gaseous Diffusion Plant, ETTP-700, Bechtel Jacobs Company LLC, Oak Ridge, TN.

SAIC 1999. Lotopic Distribution of Contamination Found at the U. S. Department of Energy Gaseous Diffusion Plants, 143.19991103 .002 , Oak Ridge, TN, October 1999.

Shonka Research Associates 1999. "Rollback Program: Surcy of the Twenty-Eight Discreet Areas," Noventer.

TDEC (Tennessee Department of Envirommenl and Conservation, DOE Oversight Division) 2006. Environmental Monitoring Repont. January through December 2005, Tennessee Department of Environment and Conservation, Oak Ridge, TN.

TVA (Tennessee Valley Authority) 1998. Personal communication with Jimrny Massengill of the Tennessee Valley Authority on April 6, 1998. 


\section{APPENDIX A}

\section{REAL ESTATE ACQUISITION LETTER}




\section{PROPOSED REAL ESTATE ACTION, OAK RIDGE RESERVATION, TN FILES RESEARCH FOR HAZARDOUS SUBSTANCE ACTIVTTY}

The following statement is provided in support of guidance promulgated under Section $120(\mathrm{~h})$ of the Comprthensive Environmental Response, Compensation and Liability Act, as amended (CERCLA) 42 U.3.C 9620(h) and in support of regulations issued by the Environmental Protection Agency at 40 CFR part 373.

The undersigned has made a complete search of existing and availabie Department of Energy (DOE) records, documentation, and data within the real estate files relating to the property that is subject to the proposed fee transfes action of Parcel ED-4 at the Esst Temnessec Technology Park (ETTP) within the Oak Ridge Reservation, Ténnessee. The proposed action would result in transfer to the Heritage Center, LEC, under a 10 CFR 770 Proposel. The search conducted was considered reasonable wrth a good fatth effort expended to identify whether any hazardous substances were known to have been released or disposed of on the property. The availabie real estate records of this office do not reflect any determinable reference that hazardous substance activity as defined by Section 101(14) of CERCLA took place on or in the property during the time the property was owned by the United States of America.

Lands affected by this action are identiffed as portions of the following original acquisition tracts in which the United States of America acquired title, (having been acquired for the Atomyc Energy Commisson as a forerunner of the Department of Energy) by Civil Action No. 429 filed in the United States Distruct Court for the Eastem District of Tennessee, Northerr Division:

Parcel ED-4 is located on a portion of Tract H-720. Title to this land was vested in the United Siates of America by Declaration of Taking No. 19. Judgment on Declaration of Taking was filed for public record on February 23, 1943, in Vol. Y-5, page 138 in the Roane County Register's Office, Tennessee.

Parcel ED-4 is located an a portion of Tract H-729. Title to this land was vested in the United States of America by Declaration of Taking No. 24. Judgment on Declaration of Taking was filed for public record on March 24, 1943, in Vol. Y-5, page 438 in the Roane County Register's Office, Tennesset.

Parcel ED 4 is located on a portion of Tract H-732. Title to this land was vested in the United States of Americs by Declaration of Taking No. 19. Judgrnent on Declaration of Taking was filed for pubtic record on Febrtary 23, 1943, in Vol. Y-5, page 139 in the Roane County Register's Office, Tennesset.

Parcel ED-4 is located on a portion of Tract H-736. Title to this land was vested in the United States of America by Declaration of Taking No. 20. Judgment on Declaration of Takng was filed for public record on February 23, 1943, in Yol. Y-5, page 170 in the Roane County Register's Office, Tennessee.

This record shall be made a part of the CERCLA report currently being prepared.

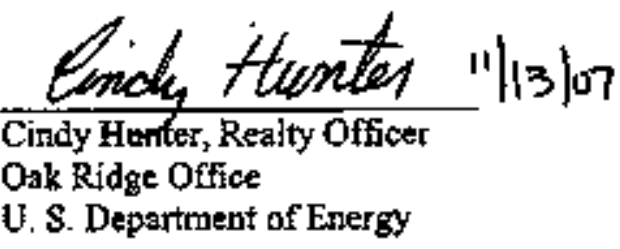

Attachament

Plat Exhibrt 


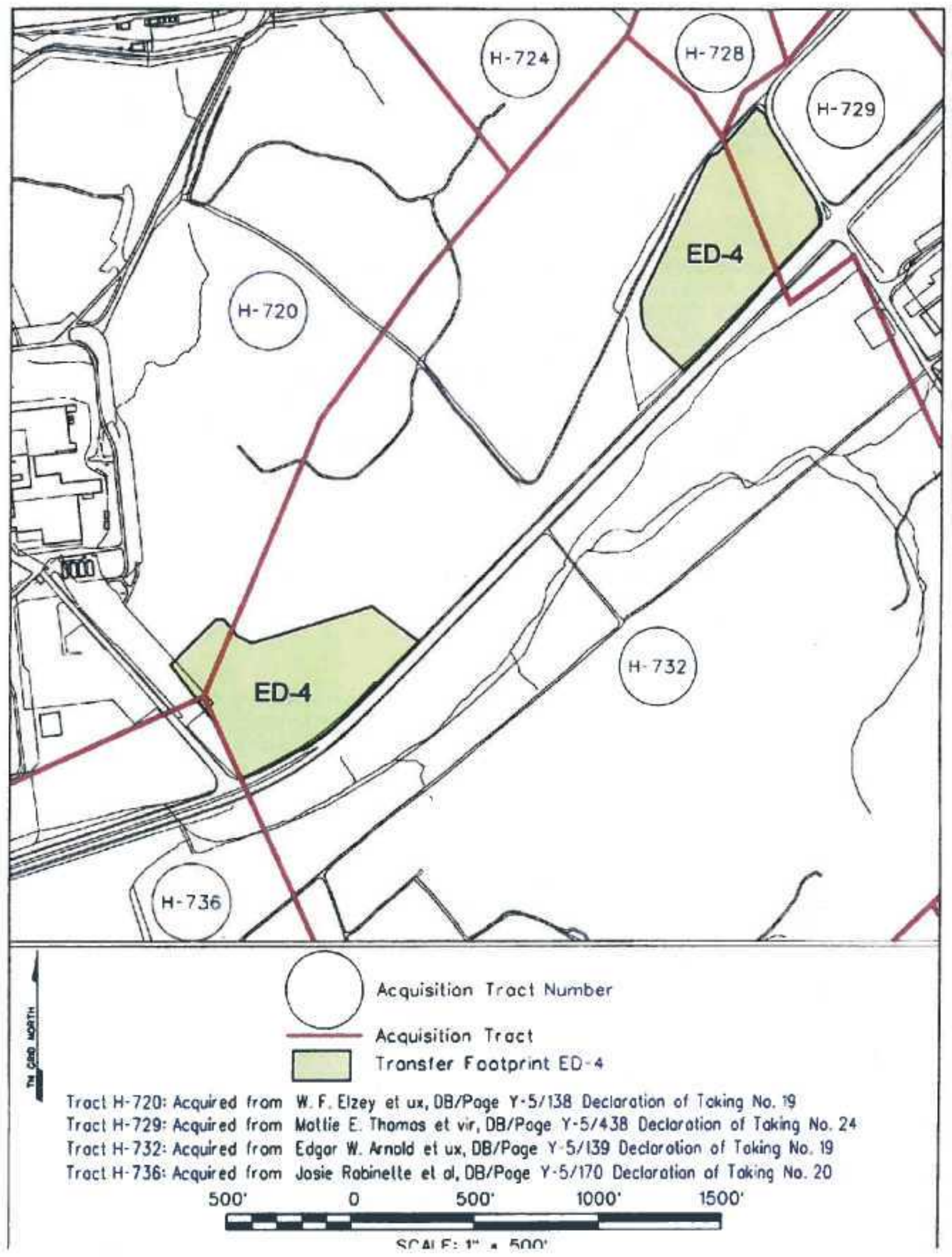




\section{APPENDLX B}

STUDY AREA MAP FROM RECORDS SEARCH 


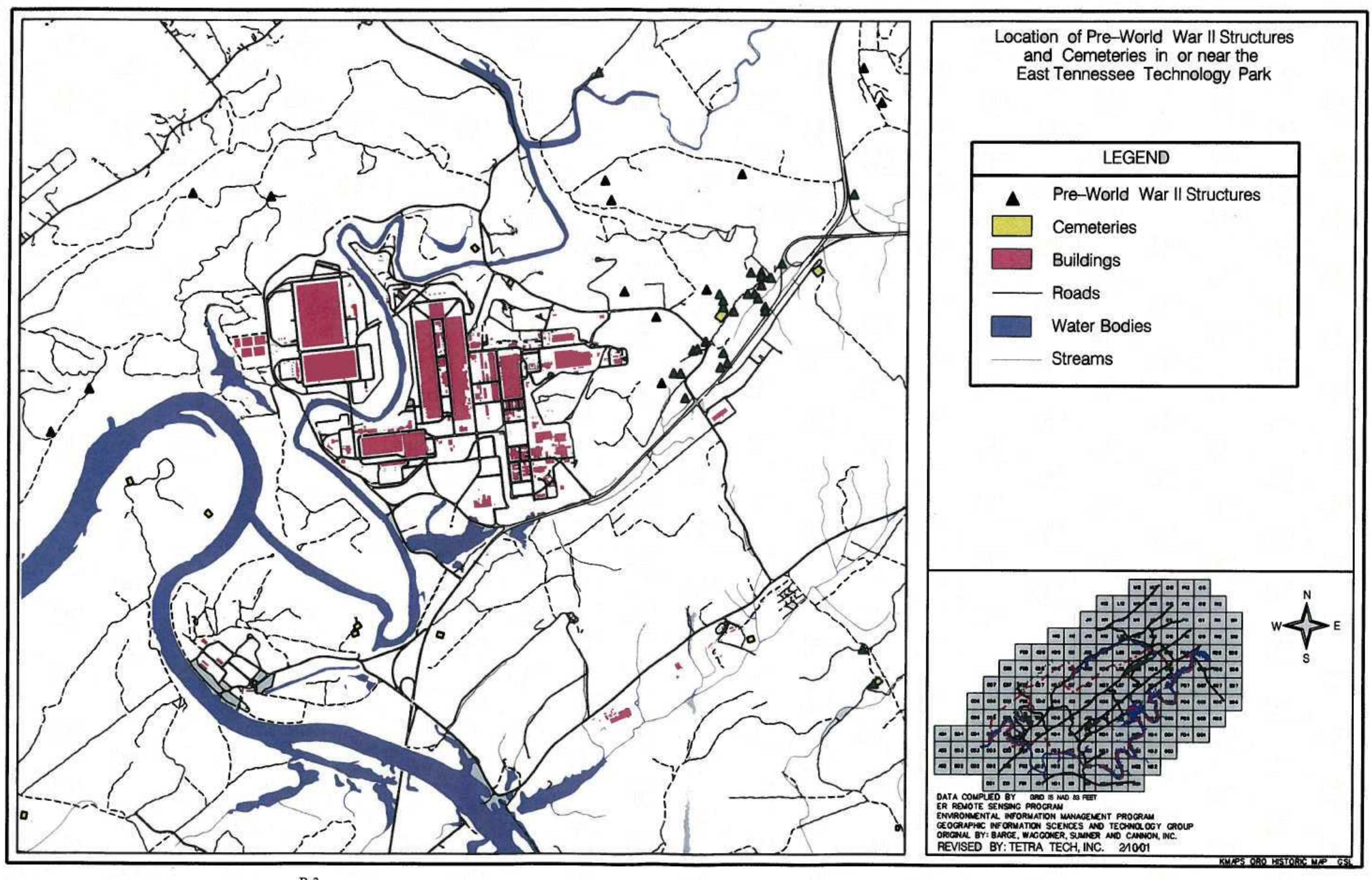


APPENDIX C

SAMPLING AND ANALYSIS PLAN FOR LAND PARCEL ED-4 AT THE EAST TENNESSEE TECHNOLOGY PARK. 


\section{Sampling and Analysis Plan for Land Parcel ED-4 at the East Tennessee Technology Park, Oak Ridge, Tennessee}

This dacument is approved foe public release per revtew by:

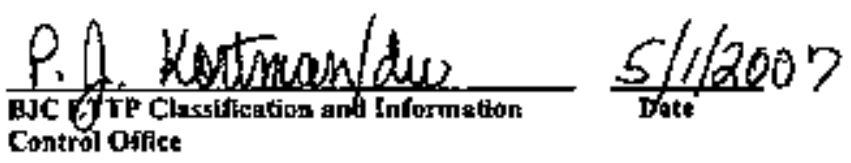




\section{SCIENCE APPLICATHONS INTERNATIONAL CORPORATION}

contributed to the preparation of this document and should not be considered an eligible contractor for its review. 


\title{
Sampling and Analysis Plan for Land Parcel ED-4 at the East Tennessee Technology Park, Oak Ridge, Tennessee
}

\author{
Date Issued-May 2007
}

\author{
Prepared by \\ Science Applications International Corporation \\ Oak Ridge, Tennessee \\ under subcontract 23900-BA-PR007U \\ under work release 000700
}

Prepared for the U. S. Department of Energy Oftice of Nuclear Fuel Supply

\author{
BECHTEL JACOBS COMPANY LLC \\ managing the \\ Envirotumental Management Activities at the \\ East Tennessee Technology Park \\ Y-12 National Security Complex Oak Ridge Nationd Laboratory \\ under contract DE-AC05-980R22700 \\ for the \\ U. S. DEPARTMENT OF ENERGY
}




\section{CONTENTS}

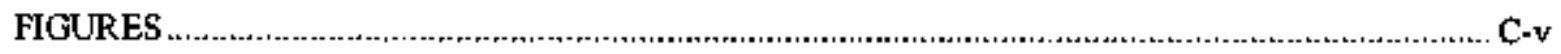

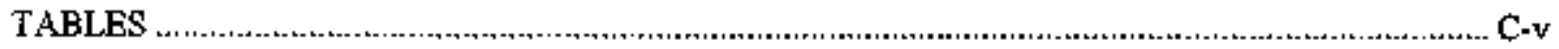

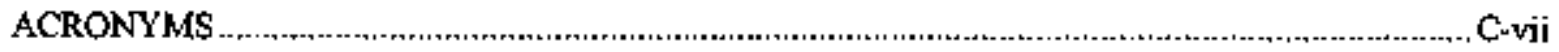

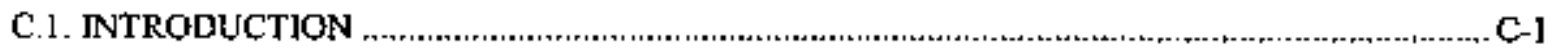

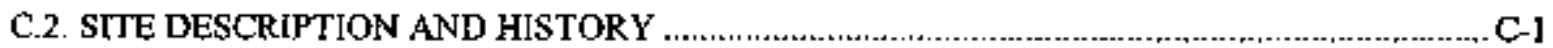

C.3. HISTORICAL DATA

C.4. OBJECTIVES

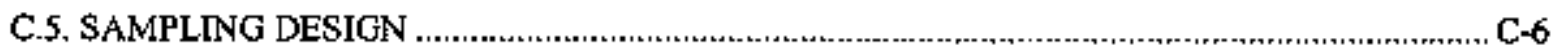

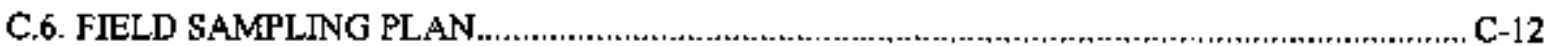

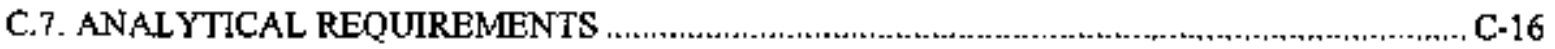

C.8. DATA MANAGEMENT AND REPORTING .............................................................. C. 77

C.9. REFERENCES

67.069(6) C-iii 


\section{FIGURES}

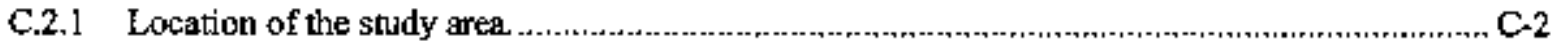

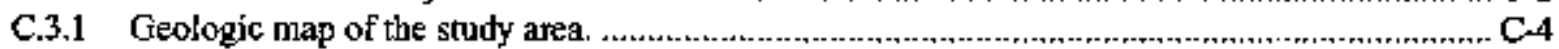

C.5.1 Proposed soil and groundwater sampling locations for ED-4.................................................. C-9

\section{TABLES}

C.3.1 Summary of VOCs detected in groundwater samples from monitoring wells in the vicinity of Parcel ED-4

C.5.1 Coordinates for Parcei ED-4 groundwater stample locations .......................................... C-8

C.5.2 Summary of sampling requirements for groundwater at Parcel ED-4 …................................. C-8

C.5.3 Summary of ground twater sampling activities for Parcel ED-4 ........................................., C-10

C.5.4 Summary of analyses for surface soil samples for Parcel ED-4 …..................................... C-11

C.5.5 Coordinates for surface soil sampling stations at Parcel ED-4 .........................................

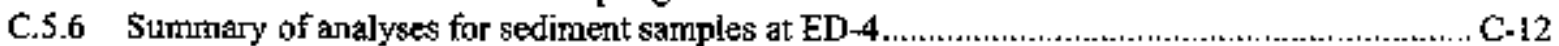

C.6.1 Container, preservation, and holding time requirenents for ED-4 groundwater samples ........ C-14

C.6.2 Container, preservation, and holding time requirements for ED-4 soil and sediment

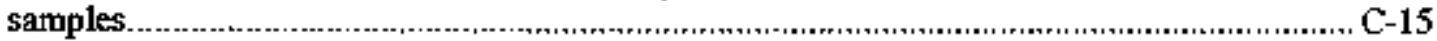

C.7.1 Analytical requirements for Parcel ED-4 groundwater, soil, and sediment samples ............... C-16 


\section{ACRONYMS}

\begin{tabular}{|c|c|}
\hline bgs & below ground surface \\
\hline CERCLA & Comprehensive Environmental Response, Compensation, and Liability Act of 1980 \\
\hline CFR & Code of Federal Regulations \\
\hline CPD & Clean Parcel Determination \\
\hline $\mathrm{DOE}$ & U. S. Department of Energy \\
\hline DPT & direct-push technology \\
\hline EPA & U. S. Environgnental Protection Agency \\
\hline ETTP & East Temnessee Technology Park \\
\hline GPS & Global Positioning System \\
\hline HVCC & Happy Valley Constuction Campsite \\
\hline ID & inside diameter \\
\hline$\mu g / L$ & micrograms per lites \\
\hline $\mathrm{NFI}$ & No Further Investigation \\
\hline ORGDP & Oak Ridge Gaseous Diffursion Plant \\
\hline PCB & polychlorinated biphenyl \\
\hline PVC & polyvinyl chloride \\
\hline QC & quality control \\
\hline R] & remedial investigation \\
\hline SAP & Sampling and Analysis Plan \\
\hline SOP & standard operating procedure \\
\hline $\mathrm{SSC}$ & sampling subcontractor \\
\hline SVOC & semivolatile organic compound \\
\hline TDEC & Ternessee Department of Environment and Conservation \\
\hline VOA & volatile organic analysis \\
\hline VOC & volatile organic compound \\
\hline
\end{tabular}




\section{C.1. INTRODUCTION}

This Sampling and Analysis Plan (SAP) presents the objectives, rationale, and the protocols for conducting groundwater, soil, and sediment sampling at a piece of land referred to as Parcel ED-4 at the East Tennessee Technology Park (ETTP), fomerly known as the Oak Ridge Gaseous Diffusion Plant (ORGDP). The study area addressed by this $\$ A P$ consists of two noncontiguous areas comprising a total of approximately 18 acres located east of the ETTP.

In order to support a Clean Parcel Determination (CPD) in accordance with Comprehensive Environmental Response, Conppensation, and Liability Act of 1980 (CERCLA) Sect 120(h)(4)(d), groundwater, soil, and sediment samples will be collected at, and adjacent to, the Parcel ED.4 study area. The potential for the U. S. Department of Energy (DOE) to make a CPD for ED-4 is supported by a No Further Jnvestigation (NFJ) determination made on land that adjoins ED-4 to the east (DOE 1997a) and to the south (DOE 1997b).

Groundwater monitoring wells have not been installed in the vicinity of Parcel ED-4, and, therefore, site-specific hydrogeologic characterization data are not available. Groundwater samples will be collected in the vicinity of Parcel ED-4, soil samples will be collected from anthropogenic features, and sediment samples will be collected from sediment accumulation areas identified turing the radiological survey. These samples will be quantified for volatile organic componnds (VOCs) and other potential contaninants that would be expected from historical operations at ETTP.

\section{C.2. SITE DESCRIPTION AND HISTORY}

The study area adiressed by this SAP consists of two noncontiguous tracts comprising a total of approximately 18 actcs. The western tract of ED-4 cncompasses approximately 8.5 aers in the northeastern quadrant of the intersection of Boulcvard Road and Highway 58 . The eastern tract cncompasses an arca of approximately 9.5 acres in the northwestern quadrant of the intersection of Blair Road and Highway 58. Highway 58 boumds both tracts of land to the south. The eastern boundary of the eastern tract follows Blair Road for ntarly 500 ft from its intersection with Highway 58 to the point at which Blair Road turns eastward. From this point the northern boundary trends to the west for approximately $300 \mathrm{ft}$ before turning to the southwest toward Highway 58 parallel to the Haul Road described below. From the Blair Road and Higdway 58 intersection, the southem boundary of the eastern tract runs approximately $800 \mathrm{ft}$ to the west along Highway 58 . This tract includes a former railroad bed and a segment of the former Wheat Road and the wetlands area near the headwaters of Mitchell Branch. The "Haul Road" actively used for truck transpott of waste materials from ETTP to the Environmental Mathagement Waste Management Facility, also bisects this tract. The western boundary of the western tract is formed by the intersection of Boulevard Road and Highway 58 and runs north for a distance of approximately $600 \mathrm{ft}$ across the corner of a parking lot. The boundary then turns northeast for approximately $300 \mathrm{ft}$ before tuming southeastward for approximately $600 \mathrm{fi}$ and then to the south to Highway 58 . The southern boundary of the westem tract runs from the Boulevard Road and Highway 58 intersection to the east for approximately $900 \mathrm{ft}$ along Highway 58. The locations of the two ED-4 tracts are depicted in Fig. C.2.1.

Aerial photographs and site maps from throughout the bistory of ORGDP indicate that this area bas largely been undeveloped woodland since federal acquisition Before that time, the land where ED-4 is located consisted primarily of forests and grasslands intermixed with large and small orchards, cropland, and pastures associated with the agriculturally based and no longer extant Wheat Community. Blacksmithing, brick making, and gristmill operations also occurred in the community. 


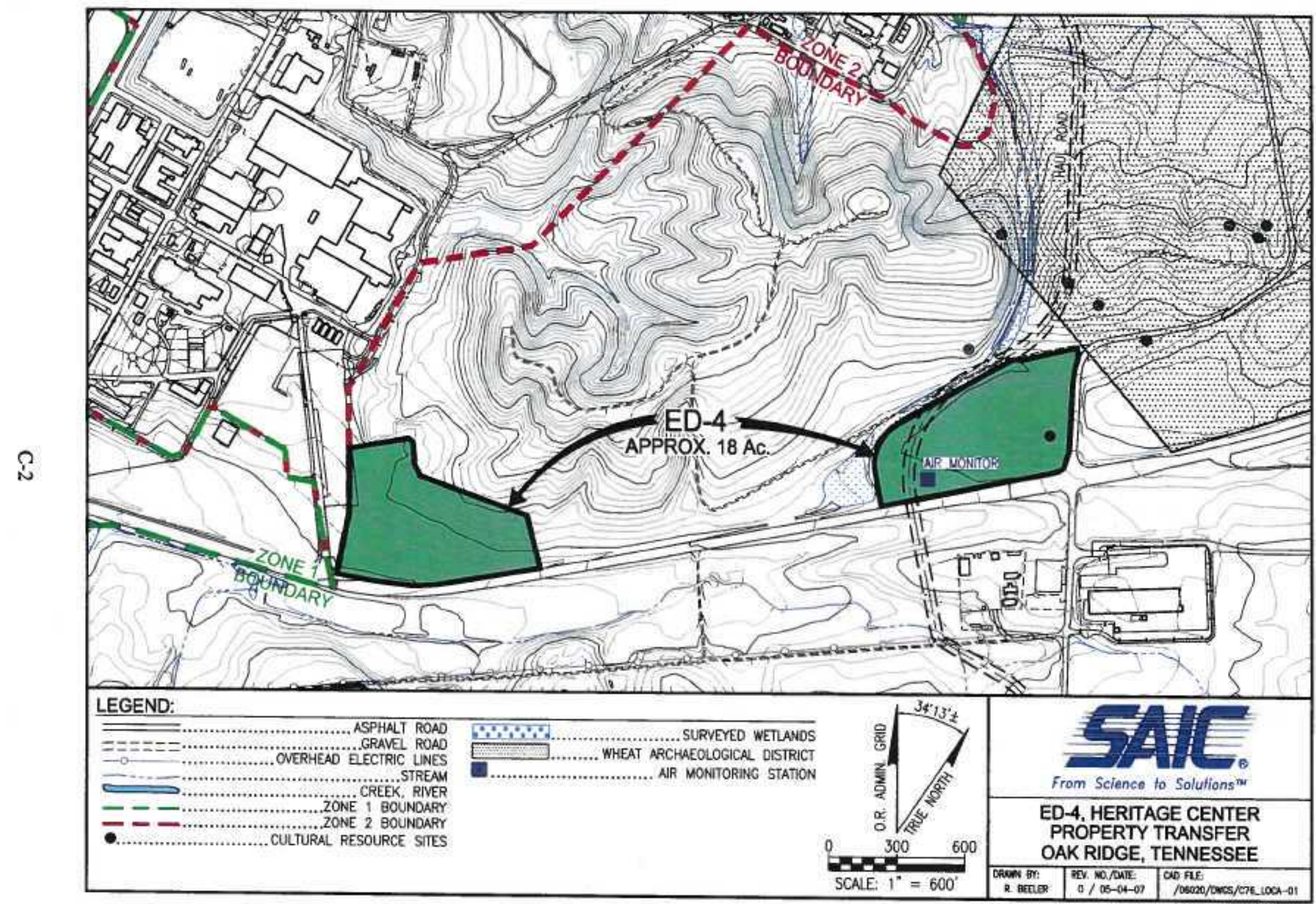

Fig. C.2.1. Location of the study area. 
Workers building the ORGDP lived in a housing area referred to as the Happy Valley Construction Campsite (HVCC), which was located south of Higbway 58 . Several of the supporting facilities for the construction workers building the ORGDP were located in the western tract of ED-4. The three HVCC buildings located within ED-4 were designated as S-12, S-22, and S-44. Historical records indicate that these buildings were the Recreation Hall (S-12), the Town Hall Camp Operations Building (S-22), and the Property Warehouse (S-44). A railroad used during site construction passed through both tracts of ED-4, as did a segment of Wheat Road. By 1963, no remnants of these former structures, including the railroad spur, are visible on aerial photograpbs.

\section{C.3. HISTORICAL DATA}

No soil samples have been collected from Parcel ED-4. Historical sampling has been conducted in the vicinity of Parcel ED-4 and has included the collection of surface soil, surface water, sediment, and groundwater from areas adjacent to ED-4. The ETTP Sitewide Remedial Investigation (RU) conducted in 1998 (DOE 1999) included the collection of two surface soil samples and two surface water samples from adjacent areas (see Fig. C.3.1). A groundwater monitoring well (UNW-125) was also installed and sampled under the activities conducted for the Ri. The Remedial Sitc lnvestigation of the HVCC (DOE 1997 ) included the collection of 13 sediment samples, of which six were collected within $300 \mathrm{ft}$ of the southern boundary of ED-4. The arza formerly occupied by the HVCC (ED-3) is also proposed for transfer as a separate action. Additional data collection is planned for the ED-3 parcel in the future. A separate SAP will be prepared in the future to address the proposed data collection effort for ED-3.

As part of the DOE's footprint reduction process, the McKinney Ridge Study Area, which incIudes an area immediately east of Parcel ED-4, was evaluated for the purpose of an NFI determination under CERCLA. The evaluation included a review of bistorical records, aerial photographs, remote sensing data, and field investigation/verification. The Tennessee Department of Environument and Conservation (TDEC) DOE-Oversight Office and U. S. Environmental Protection Agency (EPA) Region 4 approved the NF] status for the McKinney Ridge Study Area on May 28, 1998, and March 10, 1998, respectively.

Parcel ED-4 is underlain by bedrock of the Rome Formation and the Chickamauga Supergroup. Clastic bedrock of the older Rome Fombation' has been placed over the calcareous rocks of the Chickanauga Supergroup by the K-25 thrust fault, which trends generally south to noth across the westem tract of ED-4 (Fig. C.3.1). The Whiteoak Mountain Fault, which trends in a southwest-rnortheast direction along the southem boundary of Parcel ED-4 is a regional thrust fault that also places rocks of the Rome Formation in contact with rocks of the Chickamauga Supergroup.

The northeastern half of the western tract and the entire eastem tract are underlain by the Rome Formation. The lower part of the Rome Formation, which is poorly exposed in the ETTP area, generally consists of thin-bedded shale and siltstone with interbedded sandstones in variegated colors of maroon, Ereen, and yellow-brown. The upper Rome consists of maroon sandstonc, siltstone, and shale. In situ weathering of the Rome Formation yields saprolite consisting of weathered shale or siltstone, which commonly becomes more coinpetent with depth. Available exposures of this weathered saprolite in the area north of Parcel ED-4 revel numerous tight, highly fractured folds with widely ranging bedding orientations. This degree of variability precludes predictions of bedrock llowpaths in the Rome Formation at the ETTP.

\footnotetext{
' P. J. Lemiszki, 1994. Geological Kapping of the Oak Ridge K-25 Site, Oak Ridge, Tennessee, KJER-] I.
} 


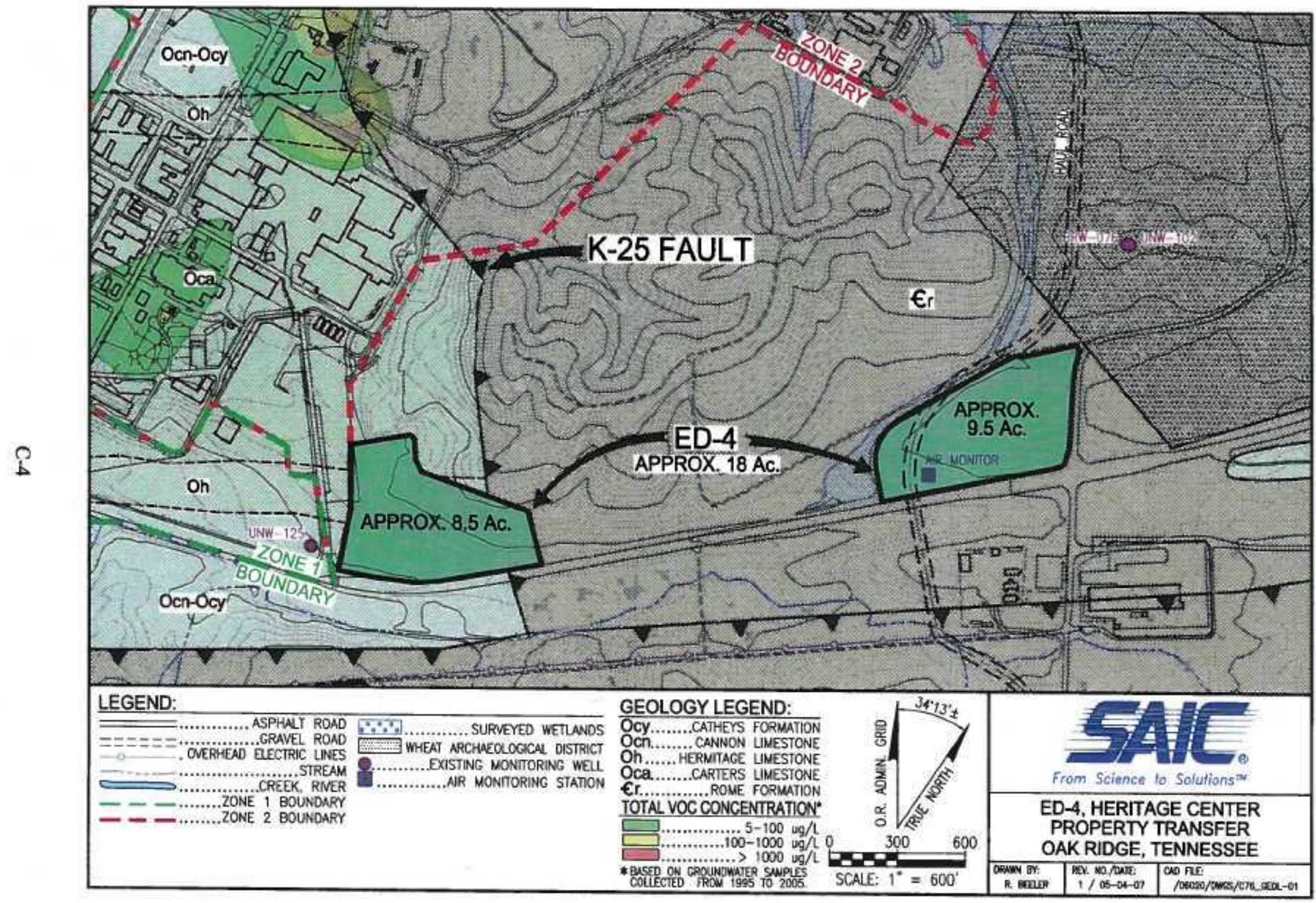

Fig. C.3.1. Geologic map of the study area. 
The Chickamauga Supergtoup fornations, which underlie the soutbwestem comer of the westem tact of ED-4, include the Carters Limestone, the Hermitage Fomation, the Cannon Limestone, and the Catheys Formation. Although less prone to karst development than the Knox Group rocks in the vicinity of the ETTP, the Chickamauga formalions art nevertheless subject to the development of karst. Solutionally enlarged fractures, joints, and bedding plants are common in exposures of Chickanauga rocks in the vicinity of ETTP. Structurally, these formations have been folded into an anticline (convex upward fold) in the vicinity of Percel ED-4 with the axis of this structure located approximately $600 \mathrm{ft}$ north of the western parcel and trends southwest-northeast. Bedding in the Chickamauge generally dips northwestward on the north side of this axis and southeastward on the south side of this axis. The western tract of Pareel ED-4 is located on the south side of the anticline axis; thus, bedding is expected to dip primarily to the southeast in the vicinity of the tract. However, movement along the Whiteoak Mountain Fault may bave caused significant disturbance of bedding orientations in the area of $\mathrm{ED}-4$.

The Carters Limestone generally consists of thick to massive beds of limestone with some medium beds and occasional interbedded argillaceous limestone and calcareous shale. Some pods and lenses of chert are present in the lower and middle parts of the formation. Although exposures were not observed during the geologic mapping of ETTP, the middle part of the Carters Limestone also contains two distinctive apple-green metabentonite beds, which tange from I to $3 \mathrm{ft}$ in thickness. Irregular, obbbly, and fossiliferous bets characterize the overlying Hermitage Formation.

Due to lack of exposures, the Cannon Limestone and Catheys Formation have been mapped as one unit at ETTP (Lemiszki 1994). The Cannon-Catheys Formation, which is pootly exposed at ETTP, generally consists of dark-gray, thick to massive beds of limestone with some thin to medium beds and shale seams and partings. Chert and siltstone fragments are common in soil developed above the Catheys Formation (Lemiozki 1994).

The water table at ETTP generally mimics topography with shallow groundwater flowing from higher topogxaphic areas to the surrounding surface water bodies. Groundwater flow through bedrock is primarily controlled by fractures, bedding planes, and hydraulic gradieat, and specific flow paths are difficult to discern. Hydrogeologic characterization data for ED-4 are limited because the property was not involved in ETTP operational activities and no contamination history exists. No groundwater moditoring wells exist in the immediate vicinity of the study area either. The oearest monitoring well (UNW-125), which is completed in the unconsolidated materials, is located approximately $150 \mathrm{ft}$ west of the western ED-4 parcel (Fig. C.3.1). In addition, a well pair consisting of an unconsolidated zone well and a bedrock welI (BRW-076UNW-102) have been installed within 500 ft to not theast of the eastern ED-4 parcel. This well pair was installed in 1993 to provide background groundwater quality data for the ETTP. The hydrogeologic characterization data presented below for ED-4 are partly based on the data fron these wells and partly based on interpolation from available ETTP sitewide information.

Because no monitoring points exist in the immediate vicinity of the study arca, depth to bedrock and depth to groundwater can only be interpolated from available data. Depth to betrock, interpolated from data in the general vicinity of ED-4, is expected to be from 12 to $30 \mathrm{ft}$ below ground surface (bgs). The depth to groundwater, interpolated from the available data, is expected to range from 5 to $25 \mathrm{ft}$ bgs depending on topographic position within the ED-4 parcels. Shallow groundwater flow is anticipated to be gencrally to the southwest toward the primary surface water bodies (K-1007-P Ponds and Poplar Creck) in this area of the ETTP.

A groundwater plume has not been identified beneath or upgradient of ED-4. The nearest groundwater plume is located approximately $700 \mathrm{ft}$ northwest of, and cross-gradient to, ED-4, and occurs in bedrock. The role of the K-25 fault on groundwater movement has not been delemined at ETTP. However, the possibility of transport of contaminated groundwater found crose-gradient and in a 
differenl sub-watershed through bedrock flowpaths to ED-4 is not considered to be likely based on hydraulic gradients, which are anticipated to transport the groundwater plume to the southwest away from ED-4. Table C.3.1 summarizes the analytical results for the VOCs detected in groundwater samples collected from the three monitoring wells located downgradient and upgradient of ED-4. The data included in Table C. 3.1 represent all of the analytical results for these wells. It should be noted that these concentrations do not represent groundwater beneath ED-4 but have been included to provide characterization data for the groundwater monitoring wells located nearest to ED-4.

VOCs have not been detected at concentrations above a federal drinking water maximum contaminant level (MCL) or TDEC domestic water supply criteria ${ }^{2}$ at any of the existing monitoring wells.

\section{C.4. OBJECTIVES}

The overall scope or objective of this SAP is to obtain data that demonstrate the absence of cheratcal and radiological groundwater, soil, and sediment contamination at ED-A to support a CPD.

\section{C.5. SAMPLING DESIGN}

The overall objectives of this $\$ A P$ are to obtain data for the two study areas comprising ED-4 that demongtrate the absence of groundwater, soil, and sediment contamination. in order to meet this overalf objective, the following ampling will be required:

- groundwater sarnples will be collected from temporary piezometers installed within the study areas;

- groundwater samples will be collected from exiating monitoring wells adjacent to the study areas;

- surface soil sarnples will be collected in the vicinity of historical anthropogenic features; and

- sediment samples will be colleted from accumulation areas identified during the radiological walkover survey.

Although groundwater impacts are nol expecled based on the history of the ED-4 area, groundwater samples will be collected to support a CPD for ED-4. The proposed groundwater sample locations bave been selected based on inferred groundwater flow directions and potential releage sites in the ED-4 area.

Groundwater samples will be collected from five (5) temporary piezometers, constructed using drive-point technology (DPT) such as Geoprobed or a similat technique, and three (3) existing monitoring wefls. The temporary piezometers bave been located to determine groundwater quality beneath ED-4 and the quality of groundwater being transported to ED-4 from upgradient areas. Piezometer 01 will provide groundwater quality in the northwestern portion of the westers tract, and piezorneter 02 will provide groundwater quality data for the area downgradjent of the former warehouse and in the vicinity of the former railroad. Piezometer 03 will provide groundwaler quality data in the downgradient portion of

\footnotetext{
${ }^{2}$ The Tennessec Department of Environment and Conservation domestic water supply criteria are equivalent to the EPA's primary dinkding water maximums contaminant Ievels (MCLs) indicaled in Table 4.2 with the exception of thloroform, which does not have a comesponding state domestic water quality criterion.
} 
Table C.3.1. Summary of VOCs detected in groundwater sumples from monitoring wells in the vicinity of Parcel ED-4

\begin{tabular}{|c|c|c|c|c|c|c|c|c|c|c|c|c|c|c|c|c|}
\hline \multirow[b]{2}{*}{ Anabyte ( $\mu \sigma / L)$} & \multirow[b]{2}{*}{ MCL } & \multicolumn{5}{|c|}{ BRW-076 } & \multicolumn{5}{|c|}{ UNW-1n } & \multicolumn{5}{|c|}{ LNW-125 } \\
\hline & & Oct-94 & Feb-95 & Sep-gh & Feb-0 & Marans & Oct-94 & Febos & Sep-95 & Dec 44 & Mar-0 & Apar 98 & Apr-9y & Sep-999 & Feh-00 & $A=5$ \\
\hline i,1,1-Trictilorgethante & 200 & $5 \mathrm{U}$ & $5 \mathrm{U}$ & 50 & 10 & $0.5 \mathrm{U}$ & $2 J$ & $5 U$ & $\leqslant \mathrm{U}$ & $0.5 \mathrm{U}$ & 0.56 & $5 \mathrm{U}$ & $\$ U$ & $5 \mathrm{U}$ & $2 \mathrm{U}$ & $2 U$ \\
\hline 1, I-Dichlorpethane & $\mathrm{NA}$ & $5 \mathrm{U}$ & $5 \mathrm{U}$ & $s \mathbf{U}$ & $\mathrm{IU}$ & $0.5 \mathrm{U}$ & 50 & $5 \mathrm{U}$ & $5 v$ & $0.5 \mathrm{U}$ & $0.33 \mathrm{~J}$ & $5 \mathrm{U}$ & $5 U$ & $5 \mathrm{U}$ & $2 \mathrm{U}$ & $2 \mathrm{U}$ \\
\hline 1,J-Dichlaroethene & 7 & $5 \mathrm{U}$ & $5 \cup$ & $5 U$ & 10 & $0.5 \mathrm{U}$ & $5 U$ & $5 U$ & $5 \mathrm{U}$ & $0.5 \mathrm{U}$ & $0.19 \mathrm{~J}$ & $5 \mathrm{U}$ & $5 \mathrm{U}$ & $5 \mathrm{U}$ & $2 \mathrm{u}$ & $2 U$ \\
\hline 2-butanone & NA & [0 U. & 100 & $10 \mathrm{U}$ & $5 U$ & 30 & 5001 & 100 & 10 & 5 UI & $5 \mathrm{U}$ & $\mathrm{NA}$ & $10 \mathrm{U}$ & $10 \mathrm{U}$ & $50 \mathrm{U}$ & $50 \mathrm{U}$ \\
\hline Hethylene chloride & 5 & $5 \mathrm{U}$ & $5 \mathrm{U}$ & $5 \mathrm{U}$ & IU & $0.5 \mathrm{U}$ & sU & $\$ U$ & $5 \mathrm{U}$ & $0.5 \mathrm{UJ}$ & $0.5 \mathrm{U}$ & $5 \mathrm{U}$ & $11 \mathrm{U}$ & 43 & $2 \mathrm{U}$ & $2 U$ \\
\hline Toluene & 1000 & $5 \mathrm{U}$ & $5 U$ & $5 \mathrm{U}$ & 10 & $0.5 u$ & 38 & $5 U$ & $5 \mathbf{U}$ & $0.5 \mathrm{U}$ & $0.5 \mathrm{U}$ & 11 & $5 U$ & su & $2 \mathrm{U}$ & $2 \amalg$ \\
\hline Trichloroethene & 5 & $\$ U$ & $5 \mathrm{v}$ & SU & 10 & $0.5 \mathrm{U}$ & $5 \mathrm{U}$ & 50 & $3 \mathrm{U}$ & $0.5 \mathrm{U}$ & $0.5 \mathrm{U}$ & $3 J$ & $5 \mathrm{U}$ & $5 \mathrm{u}$ & $2 \mathrm{U}$ & $2 \mathrm{U}$ \\
\hline
\end{tabular}

BR $W=$ bedrock well

I = estumated concentrat $\mid 0 m$

MCL = ruxirnum condmminant level

NA = NoN appticable or not ausilable

$\mathrm{U}=$ alzalyue nol delecled al indrated encentration

$\mathrm{UJ}=$ analyte not derected al indicaled concentralpon and concentration E esinnated

UNW = unconsol |litated zone well

VOC $=$ yolatile organic compound

2

$\mu y / L=$ microgerams per liter 
the eastern tract, and piezometer 05 provides groundwater quality data for the area near the wetlands and former railroad in the nortbwestern portion of the eastern tract. The 04 location will provide data for groundwater upgradient of the eastern tract The exisling monitoring wells will provide additional upgradient and downgradient groundwater quatity tata for ED-4. Collection of samples from the temporary piezoneters will be accomplished using non-dedicate mini-bailers or peristaltic pumps one to two days after piezoneter installation. The existing wells will be sampled using micropurge, low-flow sample collection techriques using non-dedicated bladder pumps. The temporary piezometers will be designated 01 to 05 and the existing monitoring wells to be sampled will include BRW-076, LNNW-102, and UNW-125. The groundwater sampling locations are presented in Fig. C.5.1. The locations for the proposed temporary piezometers in latinde/longitude and Tennesse State Plane coordinales are indicaled in Table C.5.1.

Table C.5.1. Coordinates tor Parcel ED-4 groundwater gample kocations

\begin{tabular}{|c|c|c|c|c|}
\hline $\begin{array}{c}\text { Sample station } \\
\text { number }\end{array}$ & Latítude & Longitade & $\begin{array}{c}\text { Easting } \\
\text { State Plane } \\
\end{array}$ & $\begin{array}{l}\text { Northing } \\
\text { State Plane } \\
\end{array}$ \\
\hline NS-OI-GW-ED4 & $35^{\circ} 55^{4} 40.32^{n}$ & $84^{\circ} 22^{\prime} 59.12^{\mathrm{H}}$ & 2447191.41 & 594372.83 \\
\hline NS-02-GW-ED4 & $35^{\circ} 55^{\prime} 38.59^{n}$ & $84^{\circ} 22^{\prime} 56.82^{\prime \prime}$ & 24477358.69 & 5844195.34 \\
\hline NS-03-GW.ED4 & $35^{\circ} \$ 5^{\circ} 55.75^{n}$ & $84^{\circ} 22^{\prime} 33.60^{\prime \prime}$ & 2449263.39 & 585968.19 \\
\hline NS-04-GW-ED4 & $35^{\circ} \$ 66^{\circ} 00.64^{m}$ & $84^{\circ} 22^{\prime} 28^{8} .08^{\prime \prime}$ & 2449709.33 & 586470.39 \\
\hline NS-05-GW-ED4 & $35^{\circ} 55^{\circ} 57.14^{n}$ & $84^{0} 22^{\prime} 37.32^{n}$ & 2448930.28 & 586097.12 \\
\hline
\end{tabular}

Samples collected from the temporary piezometers and existing monitaring wells will be analyzed for VOCs, semivolatile organic compounds (SVOCs), metals, herbicides/pesticides, gross alpha activity, gross beta activity, and uranium isotopes. Table C.5.2 provides a surnmary of the sampling requirenents for ED-4 and a summary of the total number of groundwater samples, and the andytical parameters, to be collected from the temporary piezoneters and existing monitoring wells is presented in Table C.5.3.

Table C.5.2. Summary of sampling requirements for groundwater at Pareel ED-4

\begin{tabular}{lcccccc}
\hline \multicolumn{1}{c}{ Sample number } & $\begin{array}{c}\text { Uranium } \\
\text { isotopes }^{\sigma}\end{array}$ & $\begin{array}{c}\text { Gross alpha and } \\
\text { beta zctivity }\end{array}$ & $\begin{array}{c}\text { TAL } \\
\text { Metals }\end{array}$ & $\begin{array}{c}\text { TCL } \\
\text { VOCs }\end{array}$ & $\begin{array}{c}\text { TCL } \\
\text { svoCs }\end{array}$ & $\begin{array}{c}\text { Herbidides' } \\
\text { Pesticides }\end{array}$ \\
\hline NS-01-GW-ED4 & $\mathrm{X}$ & $\mathrm{X}$ & $\mathrm{X}$ & $\mathrm{X}$ & $\mathrm{X}$ & $\mathrm{X}$ \\
NS-02-GW-ED4 & $\mathrm{X}$ & $\mathrm{X}$ & $\mathrm{X}$ & $\mathrm{X}$ & $\mathrm{X}$ & $\mathrm{X}$ \\
NS-03-GW-ED4 & $\mathrm{X}$ & $\mathrm{X}$ & $\mathrm{X}$ & $\mathrm{X}$ & $\mathrm{X}$ & $\mathrm{X}$ \\
NS-04-GW-ED4 & $\mathrm{X}$ & $\mathrm{X}$ & $\mathrm{X}$ & $\mathrm{X}$ & $\mathrm{X}$ & $\mathrm{X}$ \\
NS-102-GW-ED4 & $\mathrm{X}$ & $\mathrm{X}$ & $\mathrm{X}$ & $\mathrm{X}$ & $\mathrm{X}$ & $\mathrm{X}$ \\
NS-12S-GW-ED4 & $\mathrm{X}$ & $\mathrm{X}$ & $\mathrm{X}$ & $\mathrm{X}$ & $\mathrm{X}$ & $\mathrm{X}$ \\
NS-076-GW-ED4 & $\mathrm{X}$ & $\mathrm{X}$ & $\mathrm{X}$ & $\mathrm{X}$ & $\mathrm{X}$ & $\mathrm{X}$ \\
NS-TB1-GW-ED4 & & & & $\mathrm{X}$ & & \\
NS-TB2-GW-ED4 & & & & $\mathrm{X}$ & & \\
NS-01D-GW-ED4 & $\mathrm{X}$ & $\mathrm{X}$ & $\mathrm{X}$ & $\mathrm{X}$ & $\mathrm{X}$ & $\mathrm{X}$ \\
NS-7GD-GW-ED4 & $\mathrm{X}$ & $\mathrm{X}$ & $\mathrm{X}$ & $\mathrm{X}$ & $\mathrm{X}$ & $\mathrm{X}$ \\
NS-ER1-GW-ED4 & $\mathrm{X}$ & $\mathrm{X}$ & $\mathrm{X}$ & $\mathrm{X}$ & $\mathrm{X}$ & $\mathrm{X}$ \\
NS-ER2-GW-ED4 & $\mathrm{X}$ & $\mathrm{X}$ & $\mathrm{X}$ & $\mathrm{X}$ & $\mathrm{X}$ & $\mathrm{X}$ \\
\hline
\end{tabular}

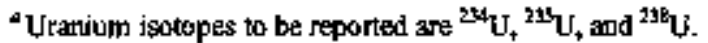

SVOC = semivolatile organit compound.

TAL $=$ Target Anelyte List.

TCL = Targen Compourt L L jat

VOC $=$ volatile orgatic compoumd 


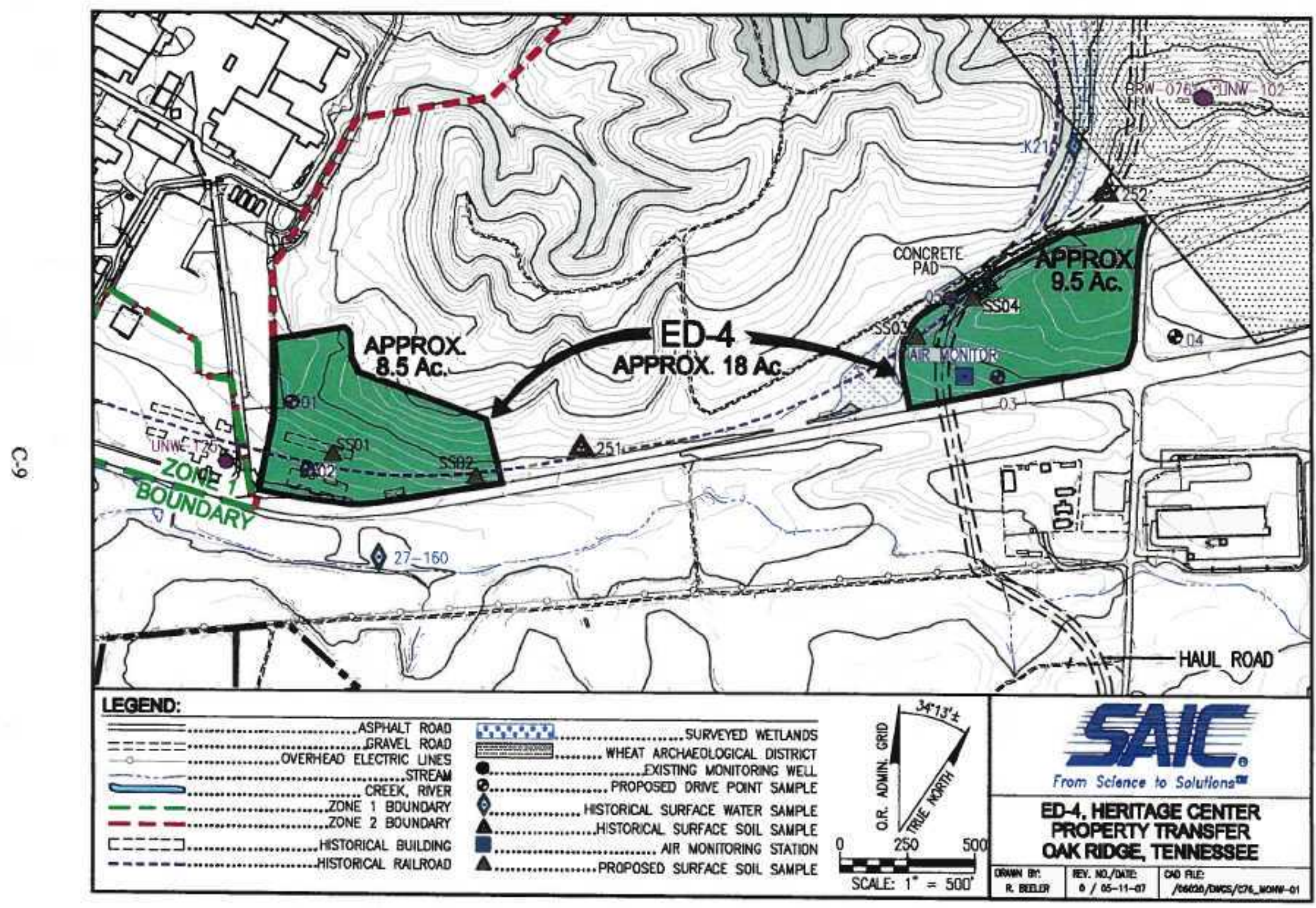

Fig. C.5.1. Proposed seil and grouadwater sampling locations for ED-4. 
Table C.5.3. Summory of groundwater sampling activities for Parcel ED-4

\begin{tabular}{|c|c|c|c|c|c|c|c|c|c|c|c|}
\hline & & \multicolumn{4}{|c|}{$\begin{array}{c}\text { Quantity of } \\
\text { semples }\end{array}$} & \multicolumn{6}{|c|}{ Rexuired analysis } \\
\hline & & $\begin{array}{l}\frac{5}{5} \\
\frac{5}{5} \\
\frac{5}{5} \\
\frac{5}{6}\end{array}$ & 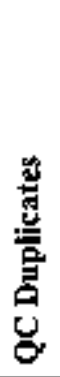 & 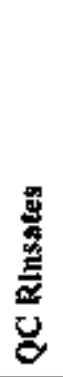 & 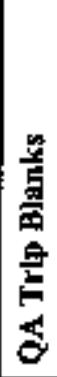 & 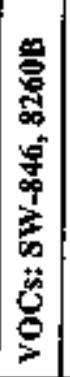 & 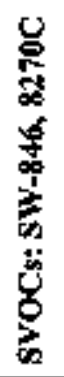 & 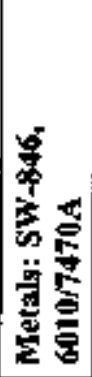 & 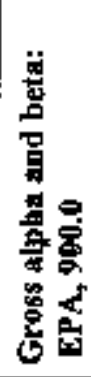 & 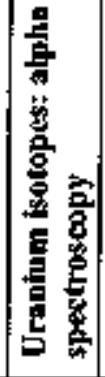 & 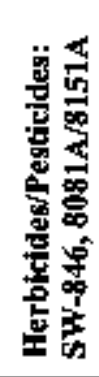 \\
\hline $\begin{array}{c}\text { DPT } \\
\text { (Temporary } \\
\text { piezometers) }\end{array}$ & Parcel ED-4 & 5 & 1 & 1 & 1 & 8 & 7 & 7 & 7 & 7 & 7 \\
\hline $\begin{array}{l}\text { Existing } \\
\text { monitoring } \\
\text { pells }\end{array}$ & $\begin{array}{l}\text { BRW-076, } \\
\text { UNW-102, and } \\
\text { UNW-125 }\end{array}$ & 3 & 1 & 1 & 1 & 6 & 5 & 5 & 5 & 5 & 5 \\
\hline
\end{tabular}

BRW = bedrock well.

DPT = direct-push technology.

EPA = U. S. Environmentel Protection Agency.

$Q A=$ quality assurmance.

$Q C=$ quality control.

$S V O C=$ semivolatile organic compound

UNW = unconsolidated zone well.

VOC = volatile organic comphound,

Soil samples will be collected from the vicinity of anthropogenic features identified from historical aerial photographs and maps, and features identified during a walkover assessment of the parcel. As indicated in Chap. C.2, limited historical operations were localed in the ED-4 area. The operations with the greatest potential to contribute contamination are likely to be incidenial spills and leaks related to the storage and bandling of materials at the former HVCC warehouse, and transport of materials over the former railroad spur. A walkover assessment of the parcel has also identified a concrete pad and debris in the eastern tract of ED-4. The contaminants most likely to have resulted from activities in these areas include VOCs, SVOCs, melals, and radionuclides. Asbestos may also be of concern due to its use in bujlding materials at that time. These contaminants, if present, would be expected primarily in surface soils.

Biased sampling of surface soils will be perforned in the vicjnity of the former warehouse, at selected locations along the railroad, and from the concrete pad. The proposed sample locations are indicated in Fig. C.5.1. All samples will be collected to a depth of 0 to $0.5 \mathrm{ft}$ bgs. Thest samples ( $\$ 501$, SS02, \$\$03, and \$\$04) will be analyzed for VOCs, SVOCs, polychlorinated biphenyls (PCBs), metals, and baseline radionuclides. In addition, locations $\mathrm{SSOl}, \mathrm{SS02}$, and $\mathrm{SSO4}$ will also undergo analysis for pesticides/hesticides and asbestos, and SSO1 will also be analyzed for transuranic radionuclides. Table C.5.4 surnmarizes the soil sampling requirentents for Parcel ED-4. Table C.5.5 provides coordinates for the surace soil sample locations to allow the field sampling crew to locate them using Global Positioning System instrumentation. 
Tahle C.5.4. Summary of 2a:alyses for surface soil samples far Parcel ED-4

\begin{tabular}{|c|c|c|c|c|c|c|c|c|}
\hline Sample station & Metals & VOC6 & SVOCs & PCBs & $\begin{array}{l}\text { Pesticides' } \\
\text { Berbitides }\end{array}$ & Asbestos & $\begin{array}{c}\text { Baseline } \\
\text { radtonuctides }\end{array}$ & $\begin{array}{c}\text { TRU } \\
\text { isotopes' }\end{array}$ \\
\hline NS-S\$O1-ED4 & $x$ & $x$ & $\mathrm{X}$ & $\mathrm{X}$ & $\mathrm{X}$ & $\mathrm{x}$ & $\mathrm{X}$ & $x$ \\
\hline NS-SS02-ED4 & $\mathrm{x}$ & $\mathrm{X}$ & $\mathrm{x}$ & $\mathrm{x}$ & $\mathrm{x}$ & $\mathrm{x}$ & $\mathrm{x}$ & \\
\hline NS-SS03-ED4 & $\mathrm{x}$ & $x$ & $\mathrm{x}$ & $\mathrm{x}$ & & & $\mathrm{X}$ & \\
\hline NS-S\$04-ED4 & $\mathrm{x}$ & $\mathrm{X}$ & $\mathrm{x}$ & $\mathrm{x}$ & $x$ & $x$ & $x$ & \\
\hline NS-SS01D-ED4 & $\mathrm{X}$ & $\mathrm{X}$ & $x$ & $x$ & $\mathrm{x}$ & $\mathrm{x}$ & $\mathrm{x}$ & $\mathrm{x}$ \\
\hline NS-S\$ERI-ED4 & $\mathrm{x}$ & $\mathrm{X}$ & $\mathrm{x}$ & $\mathrm{x}$ & $x$ & & $\mathrm{X}$ & \\
\hline NS-SSTB1-ED4. & & $\mathrm{X}$ & & & & & & \\
\hline NS-SSFBl-ED4 & & $\mathrm{x}$ & & & & & & \\
\hline
\end{tabular}

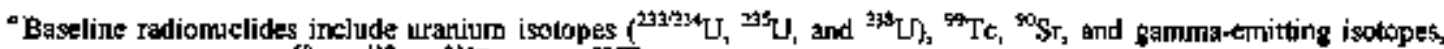

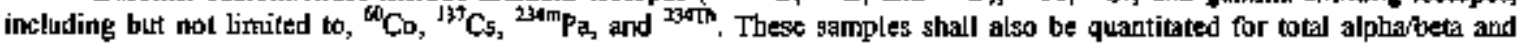
tomma activity.

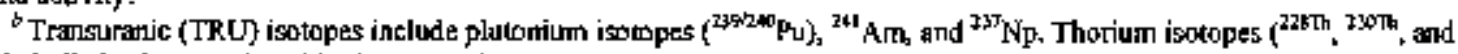
${ }^{13}$ Thi) shall also be quantilated in these samples.

$\mathrm{PCB}=$ polychlorinaled biphenyl.

SVOC = semivolatile organit compound

$\checkmark O C=$ yolatile organic compound.

Table C.5.5. Coordingtes for surface soil sampllng stations at Parcel ED-4

\begin{tabular}{|c|c|c|c|c|}
\hline Sample ID & Latitude & Longltude & State Planar East & State Planar North \\
\hline NS-SSOHI-ED4 & $35^{\circ} 55^{\prime} 39.58^{11}$ & $84^{\circ 22} 156.28 "$ & 2447401.20 & 584296.35 \\
\hline NS-SS02-ED4 & $35^{\circ} 55^{\prime} 41.86^{\prime \prime}$ & $84^{\circ} 22^{\prime} 50.42^{\prime \prime}$ & 2447879.18 & $\$ 84 \$ 34,73$ \\
\hline NS-SSO3-ED4 & $35^{\circ} 55^{\prime} 5525^{\prime \prime}$ & $84^{\circ} 22^{\prime} 37.60^{\prime \prime}$ & 2448910.99 & 585906.01 \\
\hline NS-S\$04-ED4 & $35^{\circ} 55^{\prime} 57.57^{n}$ & $84^{\circ} 22^{\prime} 36.48^{\prime \prime}$ & 2449023.86 & 586147.92 \\
\hline
\end{tabular}

One field duplicate will be collected at sampling location $\mathrm{SSOL}$. The field duplicate will be designated by the sample identifier NS-SSOID-ED4. One equipment rinsate will also be collected and designated by the identifier NS-SSERI-ED4. A trip blank will accompany each rigid containet (ice chest) used to ship samples for volatile organic analysis. The trip blank will be designated as NS-SSTB1-ED4.

Sediment samples will be collected from a maximum of five locations to be identified during the radiological walkover survey. Sediment samples will be submitted to a laboratory for analysis for VOCs, SVOCs, metals, PCBs, total radioactivity, and radionuclides. Selected samples will also be analyzed for herbicides, ptsticides, and astestos. Table C.5.6 provides a sumrnary of the analyses to be performed on the sedirnent samples. 
Table C.5.6. Snmmary or analyses for setiment samples at ED-4

\begin{tabular}{|c|c|c|c|c|c|c|c|c|}
\hline $\begin{array}{c}\text { Sample } \\
\text { Number }\end{array}$ & Metals $^{a}$ & $\mathrm{VOCs}^{\mathrm{t}}$ & SVOCs & PCBs & Asbestos & $\begin{array}{l}\text { Total } \\
\text { ectivity }\end{array}$ & $\begin{array}{l}\text { Herbicide } \\
\text { Pesticide }^{r}\end{array}$ & $\begin{array}{c}\text { Bapeline } \\
\text { radionuclides }\end{array}$ \\
\hline NS-0I-SD-ED4 & $x$ & $\mathrm{X}^{*}$ & $\mathrm{X}$ & $x$ & & $x$ & $\mathbf{X}$ & $\mathrm{X}$ \\
\hline NS-02-SD-ED4 & $\mathrm{X}$ & $\mathrm{X}^{*}$ & $\bar{x}$ & $\mathrm{X}$ & $x$ & $x$ & $\bar{x}$ & $\bar{x}$ \\
\hline NS-03-SD-ED4 & $x$ & $X^{2}$ & $\mathrm{x}$ & $\mathrm{X}$ & & $x$ & & $\mathrm{X}$ \\
\hline NS-04-SD-ED4 & $\mathrm{X}$ & $x^{2}$ & $\bar{X}$ & $\bar{x}$ & $x$ & $\bar{X}$ & & $x$ \\
\hline NS-05-SD-ED4 & $x$ & $\mathrm{X}^{*}$ & $x$ & $\mathrm{x}$ & & $\mathrm{X}$ & & $\mathrm{X}$ \\
\hline NS-02-D1-ED4 & $\mathrm{X}$ & $\mathrm{X}^{*}$ & $x$ & $X$ & $x$ & $\mathrm{X}$ & $\mathrm{x}$ & $\mathrm{X}$ \\
\hline NS-ER-0]-ED'4 & $\mathrm{X}$ & $x$ & $\bar{x}$ & $\bar{x}$ & & $\mathrm{x}$ & $\bar{x}$ & $\mathrm{X}$ \\
\hline NS-TB-00-ED4 & & $\underline{x}$ & & & & & & \\
\hline NS-FB-00-ED4 & & $\bar{X}$ & & & & & & \\
\hline
\end{tabular}
$\mathrm{Zn}$

${ }^{\circ}$ Total metals include Al, As, $\mathrm{Sb}, \mathrm{Ba}, \mathrm{Be}, \mathrm{B}, \mathrm{Ca}, \mathrm{Cd}, \mathrm{Cr}, \mathrm{Co}, \mathrm{Cu}, \mathrm{Fe}, \mathrm{Pb}, \mathrm{Mg}, \mathrm{Mr}, \mathrm{Hg}, \mathrm{Ni}, \mathrm{K}, \mathrm{Ne}, \mathrm{Si}, \mathrm{Se}, \mathrm{Ag}, \mathrm{Tl}, \mathrm{V}$, and

${ }^{b}$ Target Compound List (TCL)-volatile arganic compounds (VOCs) and TCL-semivolatile organit compoumds (SVOCs) ate to be guantified.

"Herbicides and pesticides include chlorinated herbicides and organocilorine pesticides.

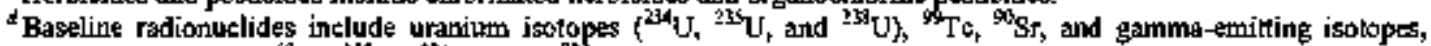
including but not limited to, ${ }^{60} \mathrm{Co},{ }^{131} \mathrm{Cs},{ }^{2540} \mathrm{~Pa}$, and ${ }^{235} \mathrm{Th}$. Uranium-235 stall also be quantified by gamma speciroscopy.

'VOC sample shall be an aliquot collected prior to compositing remaining sample materigl. The aliguot shall be collexted from the portion of the coning with the most elevated PD reading.

${ }^{i}$ Samples designated NS-ER, NSS-TB, and NS-FB are equipment rinsates, trip blanks, and field blanks, respecliyely.

\section{C.6. FIELD SAMPLING PLAN}

The various types of samples specitied in Chap. C.5 of this SAP will be obtained by the sampling subcontractor (SSC) in accordance with their contract specifications. Therefore, the descriptions of the field sempling methodologies provided in the following sections are provided only as a general synopsis of the procedures and do not supercede the SSC's contract requirements.

The groundwater investigation shall be accomplished using DPT to install tempotary piezometers, which will subsequently be sampled, In addition, three existing monitoring wells will be sampled. Groundwater samples shall be collected in accordance with the sampling subcontractor's contract specifications, "Exhibit E, Standard Specifications for Environmental Sampling." The following description of the field sampling method is provided only as a general synopsis of the sampling procedure and does not supercede the contract requirements.

Groundwater samples will be collected from five (5) temporary piezometers, constructed using DPT, and three (3) existing monitoring wells. The temporary piezometers will be placed to determine groundwater quality beneath ED-4 and the quality of grountwater being transported to ED-4 from upgradient areas. The existing monitoring wells will provide additional upgtadient and downgradient groundwater quality data for ED-4, Collection of samples from temporary piezometers will be accompilished using non-dedicated mini-bailers or peristaltic pumps one to two days after piezometer installation. The existing wells will be sampled using micropurge, low-flow sample collection techniques using non-deticated bladder purnps. The temporary piezometers will be designated 01 to 05 and the existing monitoring weils to be sempled will include BRW-076, UNW-102, and UNW-125. The groundwater sempling locations are presented in Fig. C.5.1. Samples collected from the temporary piezometers and monitoring weils will be analyzed for VOCs, $\$ \vee O C s$, metals, hebicides/pesticides, gross alpha activity, gross beta activity, and uranium isotopes. 
Temporary piezometers will be installed using a DPT drill rig. Boreholes will be drilled using the DPT until refusal of the drill rods is obtained. The temporary piezometers will be constnucted in the borehole using $\mathrm{l}$-in. inside diameter (ID) schedule 40, polyvinyl chloride (PVC) riser casing equjpped with a 5-ft PVC screen. The screens shall be manufactured with a 0.010 -in. slot size. A fine-grained silica sand (20/40 grade), to serve as a filter, shall be placed around the screen to a point at least I ft above the top of the screen. Bentonite shall be placed above the sand to serve as a temporary seal until sampling is complete. At the completion of sample collection from the lemporary piezometers, the PVC casing and screen shall be removed from the borehole and the borehole backfilled to the ground surface with bentonite.

Groundwater samples shall be collected from the temporary piezometers as soon as a sufficient amount of water for sample collection has entered the piezoneter. Groundwater samples shall be collected from the temporary piezometers using a stainless steel mini-bailer or Teflon-lined polyethylene tubing attached to a peristaltic pump.

As discussed in Chap. C.3, groundwater sampling has previously been performed at the selected monitoring wells between October 1994 and March 2005. The most recent igroundwater data at well UNW-102 indicated low estimated concentrations of chlorinated VOCs, which were not detected in the four previous sampling events at this well. Therefore, additional monitoring data will be obtained for this well to detemine the validity of the March 2005 results. The bedrock well, BRW-076, paired with UNW-102 will also be sampled to provide contemporaneous data with UNW-102. Although VOCs bave never beten detected at BRW-076, because of the placement of the shallow well, UNW-102, on a topographic high point, a bedrock transport pathway is the most likely mechanism to explain the possibility of VOCS at UNW-IO2, if they are actually present and not a sampling or laboratory artifact. Moniloring well UNW-125 is located downgradient of the western parcel of ED-4 and this well has not been sampled since Angust 2000. Thus, a groundwater sample will be collected from this well to determine current groundwater quality conditions inumedjately downgradient of the paneel. Groundwater samples from the existing wells will be collected using non-dedicated bladder pumps and micropurge, low-flow sample collection techniques. The discharge line of the bladder pumps shall be equipped with Teflon-lined polyetby lene tubing.

Groundwater samples colleted for chemical analyses from each temporary piezorneter and monitoring well shall be field-analyzed for temperature, $\mathrm{pH}$, Redox, DO, turbidity, and specific conductance. Purging of the existing monitoring wells should continue until stabilization of the field parameters has been obtained.

Samples stall be placed into appropriate laboratory containers for submittal to the laboratory for analysis inmediately upon sample collection. Sample containers will be labeled to include the sample location, sample number, sampling data and time, sampler's name, and requested analyses. Chain-of-custody procedures shall be maintained throughout the sample collection effort. Sample container preservation and holding time requirements are provided in Table C.6.I. 
Table C.6.1. Contsiner, preservation, and holding time requirements for ED-4 groundwater sampłes

\begin{tabular}{|c|c|c|c|c|}
\hline Sample hecation & $\begin{array}{c}\text { Parameters of } \\
\text { concern }\end{array}$ & Container type/vołume & Preseryation & $\begin{array}{l}\text { Holding } \\
\text { time }\end{array}$ \\
\hline \multirow{6}{*}{$\begin{array}{l}\text { Temporary } \\
\text { piezometers (01 to 05) } \\
\text { and existing wells } \\
\text { (BRW-076, UNW-102, } \\
\text { and UNW-125) }\end{array}$} & Volarile organics & (3) $40-\mathrm{mL}$ VOA vials & $\mathrm{pH}<2, \operatorname{Cool} 4^{\circ} \mathrm{C}^{\dagger}$ & 14 days \\
\hline & Semivolatile orgatrics ${ }^{c}$ & (2) 1-L A-glass-Teflon ${ }^{\mathrm{TH} *}$ & $\operatorname{Cos} 14^{\circ} \mathrm{C}$ & 7 days \\
\hline & Total metals" & 1-L Polybottle & $\mathrm{pH}<2, \mathrm{Cool} 4^{\circ} \mathrm{C}$ & 180 days \\
\hline & Herbicides/pesticides & (2) 1-L A-glass - TeflonT & $\operatorname{Cool} 4^{\circ} \mathrm{C}$ & 7 days \\
\hline & Uranium isotopes & $2 \times 1$-gal Polybottle & $\mathrm{pH}<2, \mathrm{Cool}$ to $4^{\circ} \mathrm{C}$ & I 80 days \\
\hline & Gross alpha and beta & Taken from above & $\mathrm{pH}<2, \mathrm{CoOl} 4^{\circ} \mathrm{CO}^{f}$ & I80 days \\
\hline Trip blanks & Volatile organics & (3) 40-mL VOA vials & Pre-preserved & 14 days \\
\hline
\end{tabular}

"Abalytes to be quantified are Target Compound List (TCL)-volatile orgaric compounds (VOCS).

"Preservation requirement for water samples for volatife organic compound anabysis is addition of HCl to pH $\angle 2$ end cool $4^{\circ} \mathrm{C}$.

"Analytes to be quantified are TCl-semiyolatile organic compounds (SVOCs). A-glass * amber plass.

${ }^{4}$ Holding time is 7 days to extraction and 40 days from extraction to entylyses.

'Total metels include Al, As, Sb, Ba, Be, B, Ca, Cd, Cr, Co, Ou, Fe, Pb, Me, Mr, Hg, Ni, K, Na, Se, Si, Ag, T], V, and Zn, Holding time for mercury is 28 days.

Preservation requirement for water samples for metals and radionuclides is addition of $\mathrm{HNO}$, to $\mathrm{pH} \times 2$ and $\cos 4^{\circ} \mathrm{C}$.

VOA = volatle organic analysis.

Surface soil samples at each of the locations specified in Fig. C.5.1 shall be manually collected using a hand-held auger or scoop to obtain a soil sample (core) from 0.0 to 0.5 -ft below ground surface. Upon collection of the soil sample, it shall be scanned using a PID. Samples for VOC analysis from these intervals shall be collected frora the portion of the sample that exhibits elevated PID readings from the center of the sample core. If no portion of the sanple exhibits an elevated reading, the VOC sample shall be selected randomly from the collected material at the eenter of the sample core. Samples for VOC analyses may be obtained using Encore $3^{3}$ semplers or Method $8260 \mathrm{~B}$ sampling tubes.

After collection of the VOC samples, the remainder of the collected soil mass shall be homogenized in a stainless steel bowl and transferred to appropriate sample containers. Requirements for sartele containers, preservation, and holding times for all analytes of concen from soil sampling are summatized in Table C.6.2. All sample containers shall be sealed, cleaned, and secured in accordance with the SSC's standard operating procedures (SOPs). After filling, containere will be transfered to ice chests with ice packs and a temperature check bottle. Sampling equjpment (e.g., auget, mixing bowis, etc) shail be decontarninated in accordance with the SSC's SOPs prior to reuge at other tampling stations.

Sediment samples shall be collected at locations identified during the radiological walkover survey. Sediment samples at each identified location shall be manually collected using either a shovel or hand-beld auger. Upon retrieval of the sediment material, it shall be scanned using a PID. Samples for VOC analyses shall be collected from the portion of the sediment material that exhibits the highest PID reading. If no portion of the material exhibits elevated PID readings, the sample shall be collected from the center of the material. Samples for VOC analystes may be collected using three Encore 1 samplers that are pushed into the sample material until the sampler is completely filled After collection of the sample material, the Encore(i) samplers shall be capped and placed in a plastic bag that is subsequently securely closed. The plastic bag containing the Encore samplers shall be innmediatcly transferred to an ice chest with ice packs. The bolding time for VOC samples collected using Encoretis sanplers is $48 \mathrm{hrs}$.

\footnotetext{
${ }^{3}$ Reference herein to any pecific commercial produch, process, or service by tade name, trademark, manufacturer, or otberwige, does not necessarily constitute or imply its endorsement, nocommendation, or favoring by the United States Gaverimetat or any agency thereof of its contractors of subcontractors.
} 
Alternately, the VOC samples may be collected using three laboratory pre-marked sampling tubes that are pushed into the material to the indicated interval. The sample material collected using each sampling tube shall be extruded to a laboratory pre-preserved, $40-\mathrm{mL}$ volatile organic analysis (VOA) vial. As required for Method $8260 \mathrm{~B}$, two of these VOA vials shall be pre-preserved with methanol. The third VOA vial shall be pre-preserved using sodium bisulfate solution as required by Method B260B. Holding times for VOC samples collected in this fashion are 14 days.

After collection of the VOC samples, the remaining sample material shall be transfened to a stainless steel mixing bowl, hornogenized, and transferred to the appropriate sample containers. Requirements for sample containers, preservation, and holding times for sediment samples are provided in Table C.6.2. Care shall be taken to prevent sediment from remaining in the lid threads prior to closure to prevent contarninant mignation into or from the sample. Upon filling each sample container, it shall be sealed, wiped ciean, and the lid secured in accordance with the $\$ S C$ 's $\$ O P$. After filling, the sample containers shall be transferred to an ice chest with ice packs and a temperature check bottle. Sampling equipment shall be decontaminated in accordance with the SSC's SOPs between each sampling location

Table C.6.2. Contalner, preservation, and holding time requirements for ED-4 soil and sediment samples

\begin{tabular}{|c|c|c|c|c|}
\hline Sample locstion & Par'ameters of concern & Container type/volume & Preservation & $\begin{array}{c}\text { Holding } \\
\text { time }\end{array}$ \\
\hline \multirow{15}{*}{$\begin{array}{l}\text { Locations } 01 \text { through } \\
05 \text { and S\$0t through } \\
\text { SS04 }\end{array}$} & Total metals & $\begin{array}{l}\text { 8-oz glass - Teflon } \\
\text { closurs }\end{array}$ & $\operatorname{Cool} 4^{\circ} \mathrm{C}$ & 180 days $^{6}$ \\
\hline & Metrury" & Taken from above & $\operatorname{Cool} 4^{\circ} \mathrm{C}$ & 28 days ${ }^{a}$ \\
\hline & Volatile organics ${ }^{b}$ & $\begin{array}{l}\text { Three pre-marked sample } \\
\text { tubes extruded to } 3-\times \\
40 \text {-mL pre-preserved VOA } \\
\text { vials or } 3 \text { Encore samplers }\end{array}$ & $\begin{array}{c}\text { One vial Methanot - } \\
\text { Cool } 4^{\circ} \mathrm{C} \\
\text { One vial Methanot- } \\
\text { Cool } 4^{\circ} \mathrm{C} \\
\text { One vial Nalls } \\
\text { Cool } 4^{\circ} \mathrm{C} \\
\end{array}$ & 14 days \\
\hline & Semivolatile organies ${ }^{2}$ & $\begin{array}{l}\text { 8-az A-glass - Teflon } \\
\text { closure }\end{array}$ & Cool $4^{\circ} \mathrm{C}$ & 14 days \\
\hline & PCBs (Aroclors) & Taken from above & Cool $4^{\circ} \mathrm{C}$ & 14 days" \\
\hline & Herticides/pesticides & Taken from above & $\mathrm{Cool} 4^{\circ} \mathrm{C}$ & 14 days \\
\hline & Asbestos & 50-mL plastic vial & None & Nonte \\
\hline & Uranium isotopes & $\begin{array}{l}1500 \text { g glass - Tefhon }{ }^{\text {Th }} \\
\text { closure }\end{array}$ & Cool $4^{\circ} \mathrm{C}$ & 180 days \\
\hline & ${ }^{235} \mathrm{U}$ & Taken from above & $\mathrm{CoOl} 4^{\circ} \mathrm{C}$ & 180 days \\
\hline & $\begin{array}{l}\text { Gamma-emitting } \\
\text { jsotopes' }\end{array}$ & Taken from above & $\mathrm{COO}] 4^{\circ} \mathrm{C}$ & 180 drys \\
\hline & ${ }^{90} \mathrm{St}$ & Taken from above & $\mathrm{CoO} 4^{\circ} \mathrm{C}$ & 180 days \\
\hline & ${ }^{{ }^{9} \mathrm{Te}} \mathrm{Te}$ & Taken from above & $\operatorname{Cool} 4^{\circ} \mathrm{C}$ & 180 days \\
\hline & TRU isotopes' & Taken from above & $\mathrm{Cool} 4^{\circ} \mathrm{C}$ & 180 days \\
\hline & Total alpha/beta activity & Taken from above & $\mathrm{Con} 4^{\circ} \mathrm{C}$ & 180 days \\
\hline & Total ganme activity & Taken from above & $\operatorname{Cool} 4^{\circ} \mathrm{C}$ & 180 days \\
\hline \multirow[t]{8}{*}{ Equipment rinsates } & Volatile organics & (3) 40-mL VOA vials & $\mathrm{pH}<2, \mathrm{CoO}$ to $4^{\circ} \mathrm{C}^{8}$ & 14 days \\
\hline & Semivolatile organics & (2) 1-L A-glass - Teflon ${ }^{\mathrm{T} M \mathrm{~K}}$ & $\mathrm{Cool} 4^{\circ} \mathrm{C}$ & 7 days ${ }^{h}$ \\
\hline & PCEs & Taken ftom above & $\mathrm{CoO} 4^{\circ} \mathrm{C}$ & 7 days \\
\hline & Herbicides'pesticides & Taken from above & $\mathrm{Cool} 4^{\circ} \mathrm{C}$ & 7 days" \\
\hline & Total metals & ]-L Polybottle & $\mathrm{pH}<2, \mathrm{CoOl} 4^{\circ} \mathrm{C}^{\prime}$ & 180 days \\
\hline & Gramium isotopes & (2) 1-gal Polybottles & $\mathrm{pH}<2, \mathrm{Cool} 4^{\circ} \mathrm{C}^{\prime}$ & 180 days \\
\hline & ${ }^{50} \mathrm{TC}$ & Taken from above & $\mathrm{pH}<2, \mathrm{CoOl} 4^{\circ} \mathrm{C}^{\prime}$ & 180 days \\
\hline & ${ }^{30} \mathrm{Sr}$ & Taken from above & $\mathrm{pH}<2, \operatorname{Cool} 4^{\circ} \mathrm{C}^{\prime}$ & 180 days \\
\hline
\end{tabular}


Table C.6.2. Container, preservation, and holding time requirements for ED 4 soil and sedíment samples

\begin{tabular}{|c|c|c|c|c|}
\hline Sample location & Parameters of cuncern & Contalner typervolume & Preservation & $\begin{array}{l}\text { Holding } \\
\text { time }\end{array}$ \\
\hline & $\begin{array}{l}\text { TRU isotopes } \\
\text { Ganma lsotopes } \\
\text { Total activity }\end{array}$ & $\begin{array}{l}\text { Taken from above } \\
\text { Taken from above } \\
\text { Taken from above }\end{array}$ & $\begin{array}{c}\mathrm{Cool} 4^{\circ} \mathrm{C} \\
\mathrm{pH}<2, \mathrm{Cool} 4^{\circ} \mathrm{C}^{k} \\
\mathrm{pH}<2, \operatorname{Cool} 4^{\circ} \mathrm{C}^{k}\end{array}$ & $\begin{array}{l}180 \text { days } \\
180 \text { days } \\
180 \text { days }\end{array}$ \\
\hline Trip blanks & Volatile organics & (3) 40-mL VOA vials & Pre-preserved & 14 days \\
\hline Ficld blanks & Volatile orghnies & (3) 40 -mL VOA vials & Pre-preserved & 14 days \\
\hline
\end{tabular}

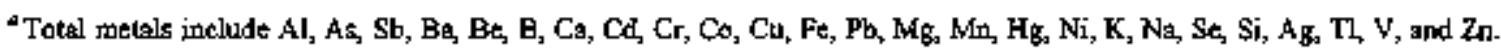
Holaitig time tor mersury is 28 deys.

${ }^{\dagger}$ Volatile organic sample ahall be an aliquot-collected before compositing material firpm the specific depth interval. Analytes to be quatilified arc Target Compoustd List (TCL)-solatile organic compounds (VOCs).

Analy tes to be quentified are TCL-semivoletile orgerric compounds (SVOC) ). A-glass = amber glass.

${ }^{6}$ Holding tisne is 14 drys to extraction and 40 days fiom extraction to analyber.

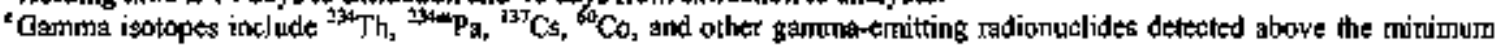
detectable aclivity. Uranium-235 shall also be quanlified by both alphe and gamma spectroscopy.

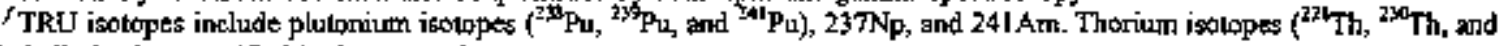
${ }^{15} \mathrm{Th}$ ) shell also be quanified in these amples.

${ }^{8}$ Preservation requirement for water samples for wo]atile organic compound analysis is addition of $\mathrm{HCl}$ to plF<2 and tcol $4^{\circ} \mathrm{C}$.

"Holding time is 7 days to extration and 40 days from extraction to analyses.

'Preservation requirement for water samples tor metals and radionuclides is addition of $\mathrm{HNO}_{3}$ to $\mathrm{pH}<2$ and cool $4^{\circ} \mathrm{C}$.

PCB = polychlorinaled biphenyl.

TRU = transuranic.

VOA = yolatile orgaric analysis (or analyte).

\section{C.7.ANALYTICAL REQUIREMENTS}

Analytical protocols for the analyte groups specified for the samples collected under this SAP are indicated in Table C.7.1. Samples for chennical analyses will be measured by the relevant SW-846 Metbods. Uranium isotopes will be analyzed by alpha spectroscopy except that ${ }^{215} \mathrm{U}$ will also be measured by gamma spectroscopy.

Table C.7.J. Annlytical requirements for Pareel ED-4 groundwater, sot!, and sediment samples

\begin{tabular}{|c|c|}
\hline Parameters of concern & Analytical protocols \\
\hline Total metals ${ }^{\circ}$ & 6010 \\
\hline Mencury & 7471 \\
\hline Total volatile organics & $8260 \mathrm{~B}$ \\
\hline Total semivolatile organics & $8250 / 8270$ \\
\hline Herbicides/pesticides & $8151 \mathrm{~A} / 8081$ \\
\hline PCBs & 8082 \\
\hline Asbestos & NIOSH 9002 \\
\hline Gamma-cmitting isotopes & Gamsna spectroscopy \\
\hline Total alpha/beta activity & Radiochemical counting method \\
\hline Total ganme activity & Gamma scan non-destructive, spectrum method \\
\hline Gooss alphe and beta & $900.0 / 9310$ \\
\hline Uranium isotopes & Apha spectroscopy \\
\hline Plutonium isotopes & Alpha spectroscopy \\
\hline${ }^{241} \mathrm{Am},{ }^{237} \mathrm{~Np}$ & A]pha spectroscopy \\
\hline
\end{tabular}




\section{C.8. DATA MANAGEMENT AND REPORTING}

Data obtained from this sampling event shall be managed in accordance with the requirements of the Data Management Implementation Plan for the Reindtstrialization Program, Oak Ridge, Tennessee (BJC/OR-865). Results will be provided to EPA Region 4 and to the TDEC DOE-Oversight Office.

\section{C.9. REFERENCES}

DOE (U.S. Department of Energy) 1997a. Evaluation of McKinney Ridge Sttudy Area, Emironmental Restoration Footprint Rediction Pracess, DOE/OR/01-1511\&D1/R1, Office of Environumental Management, Oak Ridge, TN.

DOE 1997 b. Remedial Site Evaluation Report for the Happy Valky Campsite at the Oak Ridge Resenvation. Oak Ridge, Tennessee, DOE/OR/02-1654\&D1, Office of Environmental Management, Oak Ridge, IN.

DOE 1999. Remedial Investigation Report for the East Tennessee Technology Park, Oat Ridge, Tennessee, DOEIOR01-1778\&D1, Office of Environmental Management, Oak Ridge, TN.

Energy Systems (Lockheed Martin Energy Systems, Inc.) 1995. Site Descriptions of Environtmental Restoration Units at the Oak Ridge $K-25$ Site, KJER-47/R1, Lockheed Martin Energy Systems, Inc., Environtmental Restoration Division, Oak Ridge, TN, November.

EPA (U.S. Environmental Protection Agency) 1993. Test Methods for Evaltating Solid Waste Physical/Chemical Methods $\$ W 7846$. Third Edition (November 1986; Rev. 1, July 1992; Rev. 2, November 1992; and Update 1, August 1993), Oftice of Solid Waste, Washington, D.C., August. 


\section{DISTRIBUTION}

File-EMEF DMC-RC 


\section{APPENDIX D}

\section{WALKOVER ASSESSMENT WORK INSTRUCTION}


BJC/OR-2808

FINAL

\section{Walkover Assessment Instruction for Heritage Center Property Transfers at the \\ East Tennessee Technology Park, Oak Ridge, Tennessee}


SCIENCE APPLICATIONS INTERNATIONAL CORPORATION

contributed to the preparation of this document and should not be considered an eligible contractor for its review. 
BJC/OR-2808

\title{
Walkover Assessment Instruction \\ for \\ Heritage Center Property Transfers \\ at the \\ East Tennessee Technology Park, Oak Ridge, Tennessee
}

\author{
Date lssued-May 2007
Prepared by
Science Applications International Corporation
Oak Ridge, Tennessee
under subcontract 23900-BA-PR007U
under work release 000700

Prepared for the

U. S. Department of Energy

Office of Nuclear Fuel Supply

\section{BECHTEL JACOBS COMPANY LLC \\ managing the}

Environmental Management Activities at the

East Tennessee Technology Park

Y-12 National Security Complex Oak Ridge National Laboratory under contract DE-AC05-98OR22700 


\section{INTRODUCTION}

The area to be addressed by this walkover assessment instruction includes any land parcels outside of Zone 1 and 2 at the East Temessee Technology Park (ETTP). Potential areas of interest for walkover assessments shall be identified from a review of available records. The available records to be reviewed shall include aerial photographs, historical maps, and other readily available records, including employee process knowledge.

\section{PURPOSE AND OBJECTIVE}

This walkover assessment is being performed to support Clean Parcel Determinations under CERCLA 120(h)(4). Porential areas of interest identified during this assessment may be designated for soil sample collection, if deemed to be warranted. The objective of this assessment is to conduct thorough walkovers to identify areas of possible anthropogenic sources of contamination that could potentially impact the release of the property by DOE.

\section{GENERAL METHODOLOGY}

The walkover assessment shall be performed in a manner similar to the Department of Energy (DOE) Footprint Reduction Process which was employed in the late 1990's on other land areas potentially suitable for exclusion from the CERCLA cleari-up "footprint".

The walkover assessment shall be performed by conducting observational surveys of the land parcels. The survey shall focus on areas exhibiting anthropogenic impacts, such as former roads, areas of soil discoloration, areas of stressed vegetation, and areas that appear to have been previously disturbed. Professional judgment shall be used to identify other potential areas to be assessed during the walkover. Roads and trails are investigated to determine if materials may have been dumped along these routes. In addition to areas of interest identified from the record reviews and former roads and trails, additional assessment shall be performed on unique parcel features. Routes for the foot traverses shall be selected that ensure maximum coverage of the area to be assessed.

Observational information gathered during walkdowns shall be supplemented with radiological survey, groundwater sampling and the collection of sediment samples from any sediment accumulation areas, as appropriate and based on existing data needs. Separate survey and sampling plans for these activities are prepared for implementation.

Walkover teams are equipped with field notebooks or field forms, writing instruments, maps showing the area to be assessed, Global Positioning System (GPS) instrument, and survey pin flags. Areas of interest identified during the walkover assessment will be located using GPS instruments. Locations for potential sample collection shall be marked using pin flags. The coordinate system used for identifying locations of areas of interest or potential sampling locations shall be the Tennessee State Plane, 1983 North American Datum.

All aclivities conducted during the walkover shall be documented in field logbooks or on appropriate field forms. A report shall be prepared following completion of the assessment that describes the assessment methods, the activities performed, and the resuls of the assessment, incliding any soil sampling conducted. The rationale for the type of analyses requested and the analytical results for any 
sediment samples collected during this assessment shall be reported and evaluated in the environmental documentation prepared for the lease/transfer of the property.

\section{METHODOLOGY FOR PARCELS ED-3 AND ED.4}

The first two parcels to be assessed using this instruction are designated as ED-3 and ED-4, and consist of four separate tracts located adjacent to Highway 58 (Fig. 1). The two separate tracts comprising ED-3 are bounded by Highway 58 on the north for the large tract and on the south for the smaller tracl. The larger tract occupies the area formerly known as the Happy Valley Construction campsite (HVCC). The two ED-4 tracts occupy an area bounded by Highway 58 on the south and by Blais Road to the east and Boulevard Road to the west. This walkover assessment is being perfomed in conjunction with a radiological survey and groundwater and sediment sampling efforts for these parcels, which will be conducted under efforts separate from this assessment.

Euphasis for the observational survey walkover of land pareels ED-3 and ED-4 shall be placed on any antbropogenic features such as the former railroad bed sod the path of the former Wheat Road, which transected parcel ED-4, and other former rosds thst traversed both of the pareels. Existing features such as the Hawl Road, which transects portions of both ED-3 and ED-4 (Fig. I) shall also be assessed along with any other roads and trails that are identified. Additional assessment shall be performed by conducting foot traverses at a minimum of $300-\mathrm{ft}$ intervals beginning near Highway 58 and proceeding perpendicular to Highway 58 in either a northwest or southeast direction depending on the land parcel being surveyed.

The initial walkover assessment shall be conducled for the ED-4 parcel with a subsequent assessment of ED-3. A SAP has been prepared for ED-4 for the collection of groundwater samples and the collection of sediment samples from sediment accurnulation areas ${ }^{\rfloor}$. The SAP also provides sampling and analytical requirements for sediment samples obtained at ED-4. A radiological survey to be conducted at ED-4 will identify sediment accumulation areas for sample collection ${ }^{2}$. A radiological survey plan and groundwater SAP for the small area of ED-3 not already included within the existing clean parcel determination" will also be prepared. Soil samples shall be collected at areas of interest that are deternined to wartant sample collection during the walkover assessment. Soil sample collection may be warranted if evidence, such as soil discoloration, is found indicating that soils may have been impacted by activities on the parcel.

\footnotetext{
${ }^{1}$ BJC 2007. Sampling and Analysis Plan for Parcet ED-4 at the East Tennessee Technology Park, Oak Ridge. Tennersee (in preparation).

${ }^{2}$ BIC 2007, Radiological Survey Plan for the Transfer of Land Parcel ED-4 at the East Tennessee Technology Park Oak Ridge. Tennessee (in preparation).

${ }^{3}$ DOE 1997. Environmental Restoraflon Foofprint Reduction Pracess - Evaluation of West Pine Ridge Study Area, DOE/OR/01-1568\&DI, September.
} 


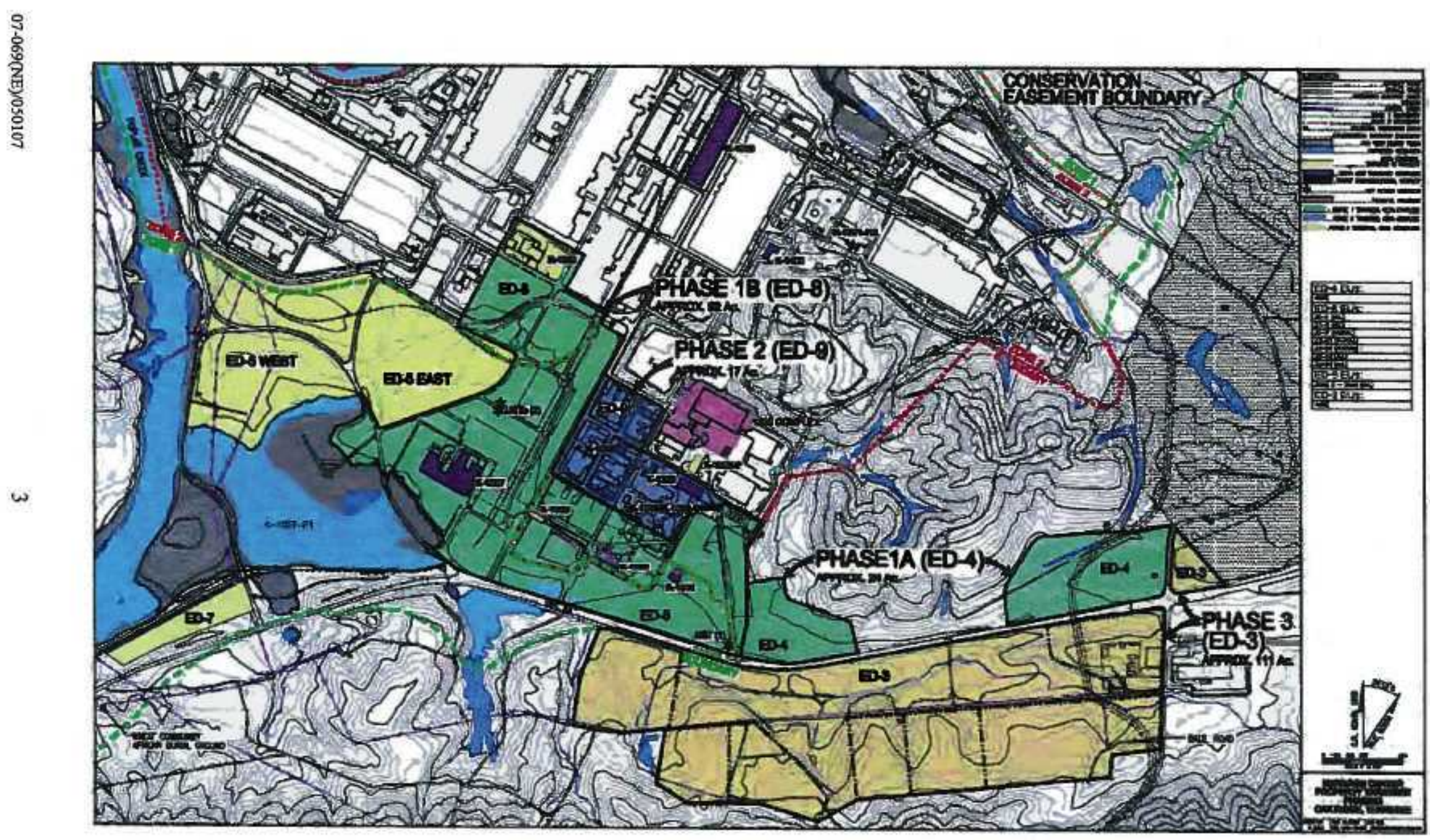

Fig. 1. Location of land parcels for Heritage Center Property Transfer 


\section{APPENDIX E}

\section{RADIOLOGICAL SURVEY PLAN FOR PARCEL ED-4}




\section{E.1. AREA TO BE SURVEYED}

Parcel ED-4, a land parcel shown in Fig. E.1, is proposed for transfer to the Community Reuse Organization of East Tennesset (CROET). Parcel ED-4 encompesses approximately 24 acres of partially wooded land that is located east of the former gas centrifige complex at the Oak Ridge Gaseous Diffusion Plant (ORGDP) in two separate tracts. Highway 58 bounds both tracts of land to the south. The eastem boundary of the eastern tract follows Blair Road for nearly $500 \mathrm{ft}$ from its intersection with Highway 58 to the point at which Blair Road nurns eastwand The southern boundary of the eastern tract rons approximately $1000 \mathrm{ft}$ along Highway 58. This tract includes a former railroad bed and the wetlands area near the headwaters of Mitchell Branch. The westem boundary of the westem tract is fomed by the intersection of Boutevard Road and Highway 58 and runs north for a distance of approxintalely $600 \mathrm{ft}$ across the corner of a parking lot. The southem boundary of the westerth tract nms approximately $900 \mathrm{ft}$ along Highway 58 . The combined area comprists approximately 24 acres. Refer to Fig. E.1 for the complete footprint of the survey area.

\section{E.2. HISTORY OF THE AREA}

Aerial photographs and site maps from throughout the history of ORGDP indicate that this area has largely been undeveloped woodland since federal acquisition. Before that time, the land in Parcel ED-4 consisted primarily of forests and grasslands intermixed with large and small orchards, cropland, and pastures associated with the Wheal Community. Blacksmithing, brick making, and gristrill operations also occured in the community.

During the Manbattan Project, the area south of Highway 58 was a portion of the campsite that provided living quarters for the construction workers building the ORGDP. Several of the supporting facilities were located in the westem tract of ED-4. The three Happy Valley Construction Camp (HVCC) buildings located within ED-4 were designated as S-12, S-22, and S-44. Historical records indicate that these buildings were the Recreation Hall (S-I2), the Town Hall Camp Operations Building (S-22), and the Property Warchouse (S-44). A railroad used during site construction passed through both tracts of ED-4, as did a segment of Wheat Road. By 1963, no remrants of these formtr structures, including the railroad spur, are visible on atrial photographs.

In the last two years, the U. S. Department of Entrgy (DOE) has construcled and is using a Haul Road for the transport of tadjological waste on the Reservation, which cuts across the eastern tract. The road is gravel and is elevated above the surrounding terrain 


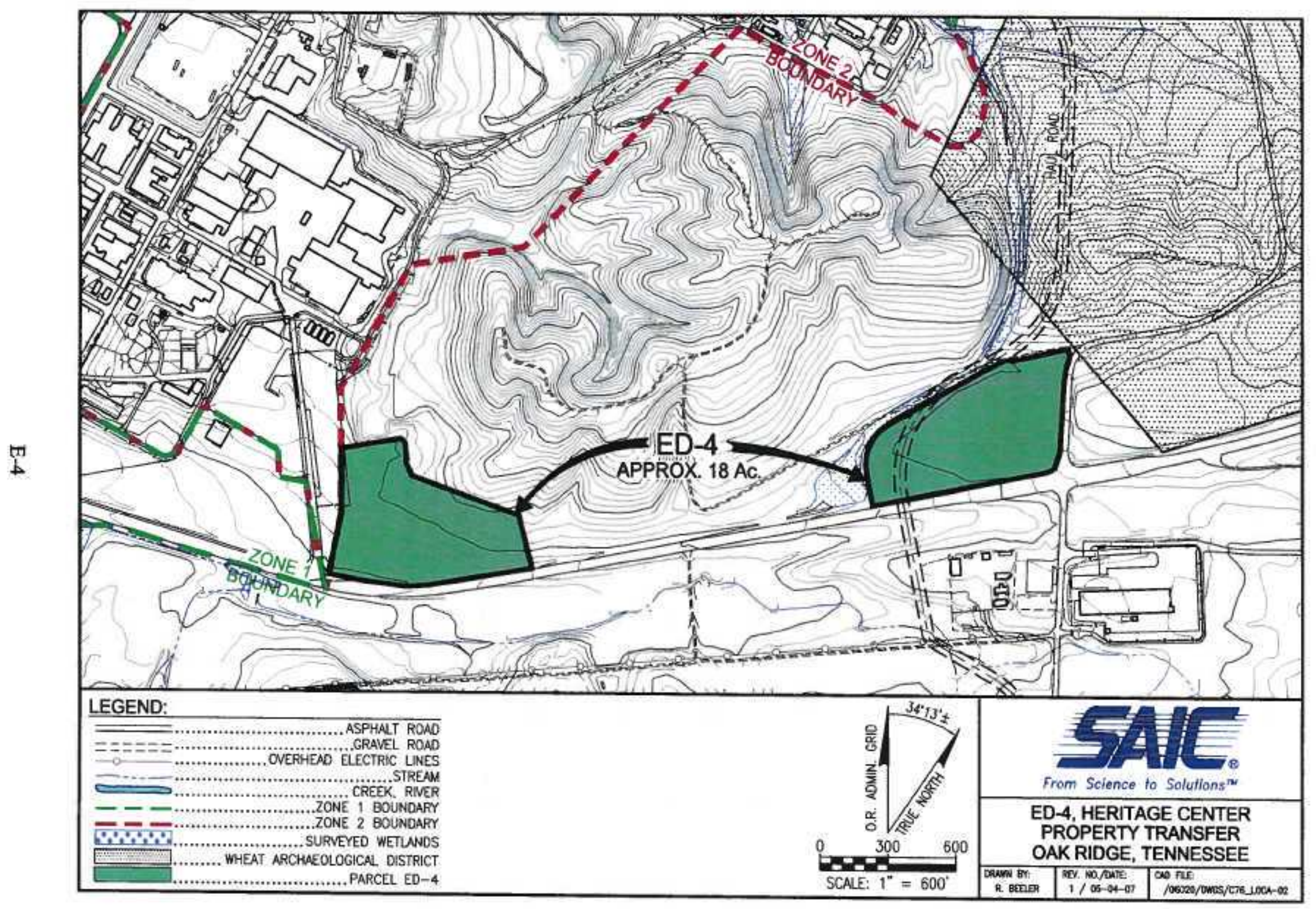

Fig. E.1. Footprint for Land Parcel ED-4. 


\section{E.3. EXISTING SURVEY AND SAMPLING DATA SUMMARY}

No survey or sampling data have been found for this land parcel. However, the eastern tract is bisected by DOE's Haul Road (used by DOE for the transport of radiological waste on the Reservation). The Haul Road footprint is eurrently being monitored Any areas exceeding 200 disintegrations per minute per 100 square centimeters $\left(\mathrm{dpm} / 100 \mathrm{~cm}^{2}\right)$ removable beta, $1000 \mathrm{dpm} / 100 \mathrm{~cm}^{2}$ total beta $20 \mathrm{dpm} / 100 \mathrm{~cm}^{2}$ removable alpha, and $100 \mathrm{dpm} / 100 \mathrm{~cm}^{2}$ total alpha are to be remediated.

The 2-in $\times$ 2-in. sodiun iodide (Nal) Ganma Scintiliator detector is the instrument of choice for these Haul Road surveys but can only be used to locate areas for survey with other instruments that can be compared to the above limits. It will be assumed that any contamination identified in connection with monitoring the Hawl Road will be rempediated prior to transfer; Haul Road aetivilies are outside the scope of this survey plan.

\section{E.4. DATA QUALITY OBJECTIVES PURPOSE}

The purpose of this survey plan is to obtain radiological survey data through the use of a scoping survey. The data gathered, combined with process knowledge, will be used to support a clean parcel determination (CPD) for title transfer of the ED-4 land parcel. The data quality objectives (DQOs) are delailed in the Design of Radiological Sturveys (DRS) documenl' found in Appendix A. However, since this parcel is outside the site boundaries and clean-up areas, the protocols bave been modified to more closely match those that have previously been used for footprint reduction efforts as noted below.

Surface contamination limits do not apply to soil-covered land parcels such as ED-4. Therefore, there are no applicable limits that apply to the data collected during the walkover survey. The purpose of this walkover survey is to visually inspect the area to collect observations and sereening data that will be used to support a CPD. The inspections will focus on identifying any anthropogenic features, delineating the boumdaries of the features, and determining if sampling of the feature is warranted For volumetric or mass meastrements, DOB Order 5400.5 mandates the use of generie guidelines for thorium and radium of $5 \mathrm{pCi} / \mathrm{g}$. For other radionuclides, guidelines must be based on specific survey unit modeling and meet a dose limit of 25 mrem modified by as low as reasonably acbievable considerations. Derived concentration guideline levels (DCGLs) will be calculated in the Sampling and Analysis Plan (SAP) for individual isotopes based on that dose limit. Therefore, it is the objective of the walkover inspection to be able to detect areas for sampling that could possibly exceed the DCGLs. Surtace water run-off and sediment accumulation areas will also be identified for sampling.

\section{E.5. MEASUREMENT TECHNIQUES/SURVEY APPROACH}

\section{E.5.1 RADHONUCLIOES OF INTEREST}

The process history of the East Tennessee Technology Park (ETTP) site indicates that uranium (natural, depleted, and/or enriched) would be the most prominent radiological contaminant potentially present in Parcel ED-4 as a result of emissions or tracking of contamination from on-site buildings.

\footnotetext{
${ }^{1}$ Desigh of Radiological Survey and Sampling to Support This Thansfer or Lease of Property on the Department of Eneriy Oak Ridge Retervation, BJCJOR-554-R1, August 2006.
} 
Uranium-235 enrichment levels from operations since the early 1960 s would be expected to be between 0.2 and $5.0 \%$. Most ETTP areas would have potentially been contaminated by tracking from entrichments of less than $3 \%{ }^{2}$

Other radionuclides $\left({ }^{60} \mathrm{Co},{ }^{137} \mathrm{Cs},{ }^{8 \times 90} \mathrm{Sr}_{2}{ }^{237} \mathrm{~Np},{ }^{90} \mathrm{Te}\right.$, and $\left.{ }^{238 / 28,9240} \mathrm{Pu}\right)$ have also been detected on-site at ETTP. These other radionuclides originated from the introduction of contaminated materials from the Oak Ridge National Laboratory and/or from the Hanford and Savannah River reactor returns uranium-reprocessing program; however, these radionuclides are expected to be found in much lower quantities than uranium and to be undetectable in this area. If radionuclides are present, it is assumed that they will be present at ratios of 1140:1 for uranium to transuranic and 350:1 for uraniurn to technetium-99 (both ratios are process-building weighted averages). ${ }^{3}$

\section{E.5.2 IDENTIFICATION OF SURVEY UNITS AND CLASSIFICATIONS}

Under the DRS protocols that are based on Muli-Agency Radiation Survey and Site Investigation Mantial (MARSSIM) guidance, survey units are classified as either non-impacted, or Class 3,2, or I based upon historical data and process knowledge. Non-impacted areas have no reasonable potential for residual contamination and require no further evidence to demonstrate compliance with the release criterion. An area is considered a Class 3 survey unit if it is not expected to have residual radioactivity levels above $25 \%$ of the DCGL. A Class 2 survey unit is expected to have, or has had, residual radionetivity levels less than the DCGL but above the criteria for Class 3. A Class 1 survey unit is expected to have, or has had, residual radioactivity levels above the DCGL.

The ED-4 land parcel will consist of two land survey units (LSUs), as shown in Table E. L. Though contanination is considered unilkely in ED-4, its proxitnity to the former ORDGP precludes its classification as non-impacted. Because of the parcel's dormant status and lack of development, except during the construction of the plant site and the current Haul Road, contamination in the eagtern tract (LSU 2) is not expected, and if present, should be at levels well below the DCGL. It has been judged that some potential for low levels of contamination exisis in LSU 1, since its westem boundary's proxímity to the formes ORGDP area increases the potential for contamination of this localized area from groundwater runoff because the property slopes in that direction. However, it is not expected that the levels would exceed $25 \%$ of the DCGL. Therefore, both ED-4 tracts will be classified as Class 3.

Table R, J. Land sqrvey units

\begin{tabular}{ll}
\hline \multicolumn{1}{c}{ Survey unft } & Class \\
\hline LSU 1 - western tract & Class 3 \\
LSU 2 - castem tract & Class 3 \\
\hline
\end{tabular}

\section{E.5.3 INSTRUMENTATION SELECTION AND SURVEY TECHNIQUES}

Refer to the DRS in Appendix A for details on instrumentation selection. NaI meters and Bicron Microkem meters will be used, as specified in this survey plan.

\footnotetext{
${ }^{2}$ Contracted Health Physics Tochniciant Training tandouts, K-25 Site, 1993.

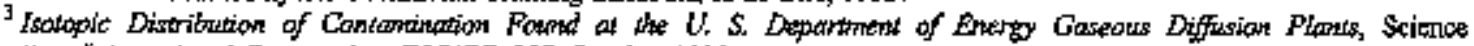
Applications Intemational Corporation, BJCIOR-257, Oetober 1999.
} 
The Walkover team should be equipped with the following:

- field notebook and writing instruments,

- maps showing the ISU and assessment locations,

- Global Positioning System (GPS) instrument for in-field locating of the assessment locations and for documenting locations of notable field observations,

- survey pin-flags,

- Bieron MicroRem(S) meter, and

- 2-in.-diameter $\times 2$-mm-thick NaI delector for surveying assessment locations and possible anthropogenic features, and sediment collection area. If a 2-in-diameter $\times 2$-mm-híck NaI detector is not available, a 2 -in. $\times 2$-in. NaI detector may be substituted.

If necessary, the visual inspections and locations of assessment points using a GPS unit may be performed by a separate tem prior to the survey measurements. If so, the assessment points and any other locations to be measured (anthropogenic, groundwater runoff, and sediment collection areas) will need to be marked in such a way that the locations can be found by the survey tearn.

For the Class 3 areas, NaI walkover survey judgment scans will be performed. Emphasis will be placed on road bed areas, suspect areas of discoloration, and other areas based on professional judgraent. Any anthropogenic, groundwater runoff, and sediment collection areas recogrized during the NaI waltover, and any scan areas detemined to bave elevated readings, will be marked, and timed-fixed NaI and dose-rate measurements will be taken at the specifically identified locations of highest scan readings. A fixed NaI and dose-rate measurements will also be taken at randomly generated points based on a supplied grid

All surveys will be performed in accordance with established Bechlel Jacobs Company LLC (BJC) Radiation Control Otganization (RADCON) procedures (e. g., scan rate, probe distance, source checks).

\section{E.5.4 AREA PREPARATYON}

All arcas will be surveyed in an "as-found" condition. Materials may be reartanged or moved to allow for survey access to areas covered by debris. Clearing of some areas by bushhog or pther means may be necessary for aceess lo some poinls.

\section{E.5.5 REFERENCE COORDINATE SYSTEM FOR SURVEY}

The DRS nomally requires random points to be generated for timed measurements for Class 3 survey umits. However, due to the modified DQOS discussed above, a sample grid with systematic measurements taken based upon a random starting point will be used. These survey grids are based upon the LSU's area such that there is approxinately one assessment point per acre. A reterence coordinate system will be used in each survey unit to reference measurements so they can be relocated/verified as needed. The reftrence coordinate system used for the anticipated transects will be the state plane coordinates. 


\section{E.6. SURVEY DESIGN}

\section{E.6.1 DETERMINATION OF THE NUMBER OF DATA POINTS}

LSUs have a prescribed number of data points (samples), at a minimum, to gather, which is basted upon the protocols described in the MARSSIM. However, there is no set number of survey measurements required for soil areas, as the determination of the LSU's conlanination level, as conpared to the DCGL, is done ușing soil sample dath not hand-held instrumentation data. It is desired to make timed $\mathrm{NaI}$ and dose rate measurements in onder to identify possible locations for representative sampling. Therefore, assessment points for timed $\mathrm{NaI}$ and dose rate measurements will be located at intersects of a survey grid whose size is based upon the LSU's area, such that there is approximately one point per acre. In addition, anthropogenic, groundwater nuoff, and sediment collection areas will be identiffed by visual inspection for biased, timed NaI and dose rate measurements.

\section{E.6.2 SURVEY PROCEDURES}

All surveys are to be performed in accordance with this survey plan, the DRS, and BJC RADCON procedures. ${ }^{+}$Note: The survey technique is covered in the DRS and will not be repeated in this plan; however, variations from, or clarifications of, the design document protocols are included below.

Many of the radionuclides found on the Oak Ridge Reservation bave natural backeround concentrations; therefore, background subtraction will be required for all direct field measurements. Sorte comparison to background levels will also be required for the scanning because only a gross signal will be measured. Material-speciffe backgrounds might be necessary for materials such as tile, brick, and cinderblock because these materiaIs contain elevated Ievels of naturally occuring radionuclides.

NaI walkover survey judgment scans will be performed. Emphasis will be placed on road bed areas, suspect areas of soil or vegetation discoloration, and other areas based on professional judgment Nal survey measurements will be performed at the fixed grid assessment points, any anthopogenic, groundwater nnoff, and sediment collection areas recognized during the NaI walkover, and any scan areas detemined to have elevated readings. (Sediment accumulation areas are those areas where overland flow and surface drainage gradients decrease and sediment may accumulate. These accumulation areas vill generally be flat or low-lying areas that would tend to accumulate run-off and any sediments.) $A$ 10-ft-diameter surface area will be scanned at each of these points with the NaI detector, and the location of the highest reading will be counted for 1 minute and the results recorded. Biased samples will be collected for laboratory analysis from any location that has a timed NaI survey reading greater than three times the established background. The basis for the "three limes rule" stems from the fact that natural backgrounds vary by up to a factor of three, depending on geology, topography, and other geometric factors. However, the data for each LSU will be teviewed to determine if other areas exist where there is a clear elevation in count rate as compared to surtounding areas but less than three times the establisbed background. Professional judgment will be used to evaluate if the specific geology, topography, and matrix (e.g., rock outcroppings, pavement, severe slopes, and brick buildings) could have caused the elevated readings. If a background specific to the geology and topography for the area can be obtained, a lower trigger level (e.g., two times the background or the $99 \%$ decision level for the NaI meter) may be used based on professional judgment.

\footnotetext{
"Primarily EH-4516, "Radioactive Contamination Control and Monitoring," found in BJC-EH-4000, Radiation Protection Poggam Description tor Bechtel Jacobs Company LLC. Oak Ridge, Tennessee. 
A sunumary of the requirements for each survey unit is found in Table E.2.

Table E.2. Summary of survey unit requireunents

\begin{tabular}{|c|c|}
\hline $\begin{array}{c}\text { Survey unit } \\
\text { type }\end{array}$ & Class 3 \\
\hline $\begin{array}{l}\text { Land } \\
\text { (LSU) }\end{array}$ & 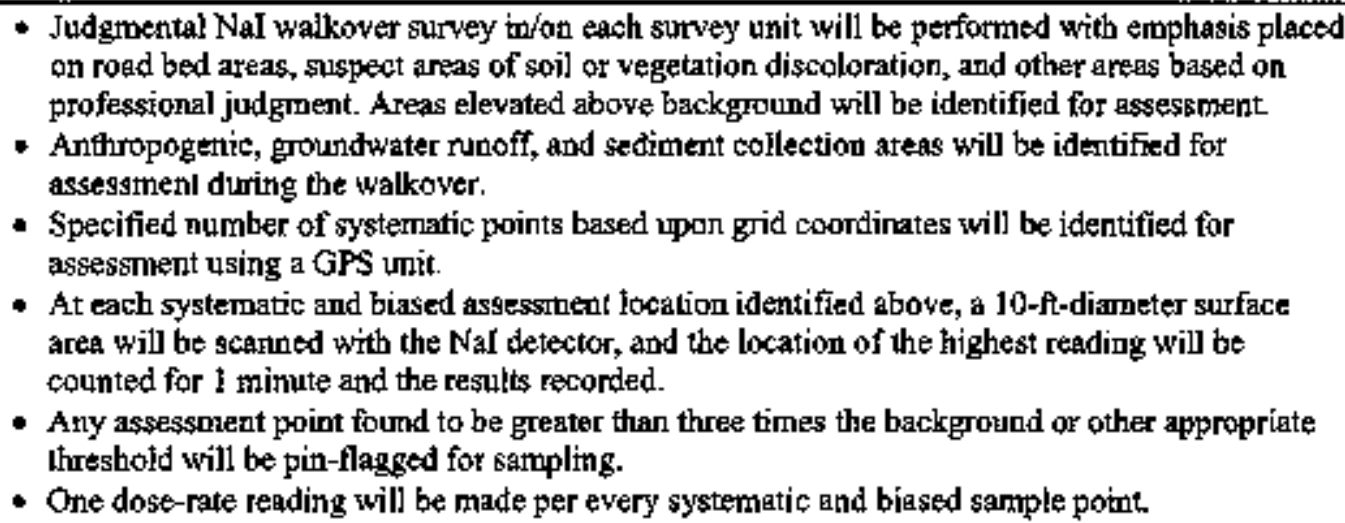 \\
\hline
\end{tabular}

GPS = Global Positioning UniL

LSU = land surycy unit.

NaI = sodjum iodjde.

\section{E.6.3 SPECIFICATION OF ASSESSMENT POHNT LOCATIONS}

Systernatic assessment points will be based on the survey grid as shown on Fig. E.2. The state plane system coordinates and longitude/latitude for each assessment point for LSU 1 are shown in Table E.3; those for LSU 2 are shown in Table E.4 and correspond to the locations shown on Fig. E.2. In addition, the walkover inspection team will identify anthropogenic, groundwater nunoff, and stediment accumulation areas, and any other areas scanned above background in the LSUS, as biased assessuent points.

Table E.3. Assesament point locations for LSU 1

\begin{tabular}{|c|c|c|c|c|c|c|}
\hline $\begin{array}{l}\text { Assessment } \\
\text { point } \\
\text { number }\end{array}$ & $\begin{array}{l}\text { Oak Rivige } \\
\text { admísistrative } \\
\text { grld } \\
\text { egsting }\end{array}$ & $\begin{array}{c}\text { Oak Ridge } \\
\text { administratdve } \\
\text { grid } \\
\text { northing }\end{array}$ & $\begin{array}{l}\text { Tenncosee } \\
\text { State grid } \\
\text { epgting }\end{array}$ & $\begin{array}{c}\text { Tennessee } \\
\text { State grid } \\
\text { northing }\end{array}$ & Latitude & Lonfitade \\
\hline$A P=01$ & 14975.00 & 33675.00 & 2447125.66 & 584403.53 & $842259 \overline{90097}$ & 355540.6293 \\
\hline$A P-02$ & 15196.22 & 33716.04 & 2447282,68 & 584564.65 & 842257.9683 & 355542.1969 \\
\hline$A P=03$ & 15016.04 & 33453.78 & 2447286.79 & $\$ 84246.5]$ & 842257.9823 & 355539.0503 \\
\hline$A P-04$ & 15237.27 & 33494.82 & 2447443.81 & $\$ 84407.63$ & 842256.0410 & 355540.6179 \\
\hline $\mathrm{AP} \sim 05$ & 15458.49 & 33535.87 & 2447600.83 & 584568.76 & 842254.0995 & $35 \$ \$ 42.18 \$ 5$ \\
\hline AP-06 & 15278.31 & 33273.60 & 2447604.93 & 584250.61 & 842254.1136 & 355539.0389 \\
\hline $\mathrm{AP}-0 \mathrm{~T}$ & 15499.54 & 33314,64 & 2447761.95 & 584411.74 & 842252.1722 & 355540.6065 \\
\hline AP-08 & 15715,97 & 33360.47 & 2447912.30 & 584574,00 & 842250.3117 & 355542.1864 \\
\hline
\end{tabular}

LSU = land survey unit. 


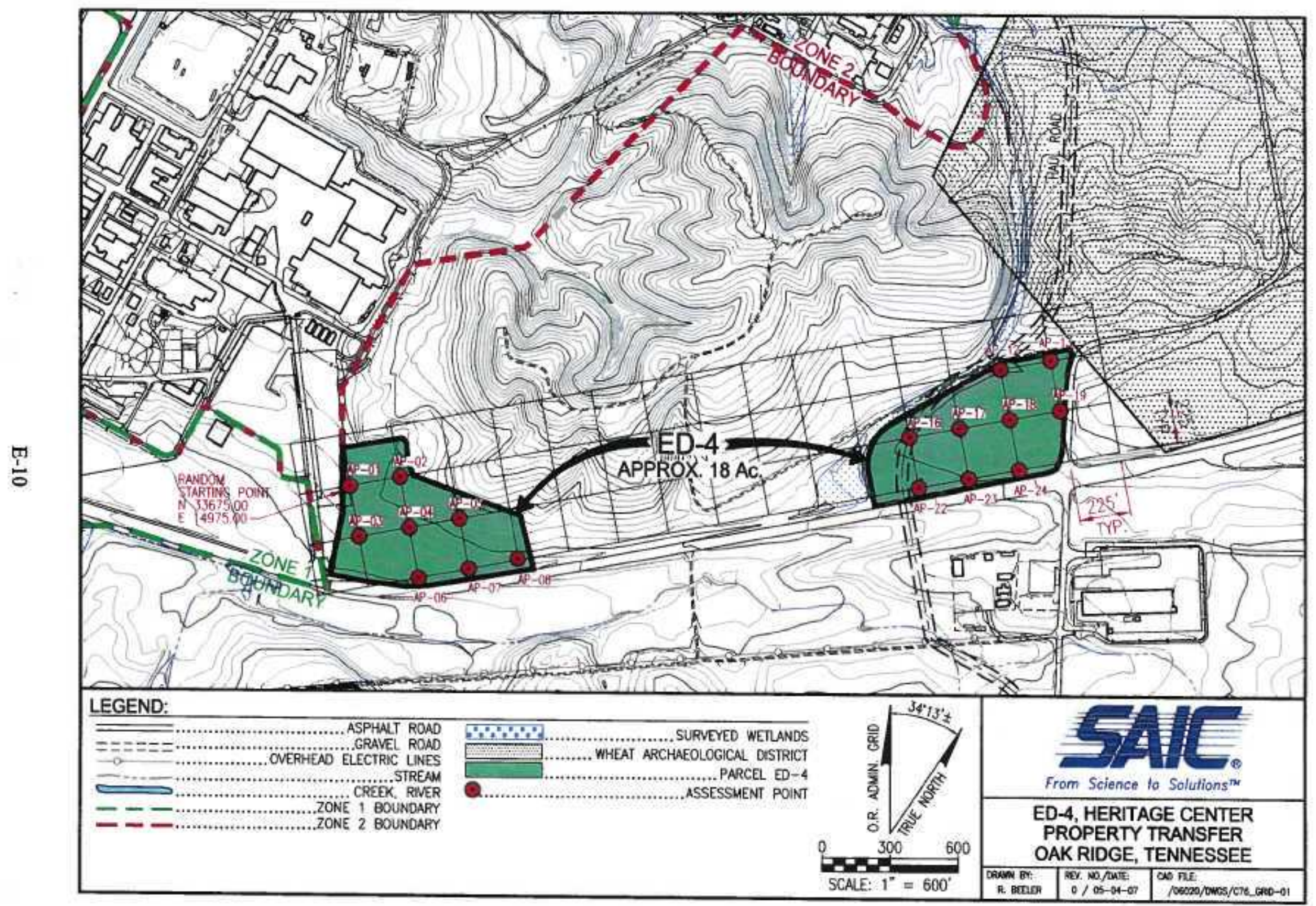

Fig. E.2. LSU assessment point locations. 
Table E.4. Assessment polnt locations for LSU 2

\begin{tabular}{|c|c|c|c|c|c|c|}
\hline $\begin{array}{l}\text { Assessment } \\
\text { point } \\
\text { number }\end{array}$ & $\begin{array}{c}\text { Oak Ridge } \\
\text { administrattue } \\
\text { prid } \\
\text { ensting }\end{array}$ & $\begin{array}{c}\text { Oak Ridge } \\
\text { administrative } \\
\text { prid } \\
\text { northing }\end{array}$ & $\begin{array}{l}\text { Tennessee } \\
\text { State grid } \\
\text { essting }\end{array}$ & $\begin{array}{l}\text { Tennessee } \\
\text { State grid } \\
\text { northing }\end{array}$ & Latitude & Langiturde \\
\hline$A P-12$ & 17850.92 & 34208.58 & 2449166.93 & $\$ 86498.15$ & 842234.6699 & 355601.0075 \\
\hline AP-13 & 18072.14 & 34249.63 & 2449323.95 & 586659.28 & 842232.7282 & 355602.5750 \\
\hline AP. 16 & 17449.52 & 3390527 & $2449014.0 \mathrm{I}$ & 586018.88 & 842236.6258 & 355556.2934 \\
\hline AP-1T & 17670.74 & 33946.31 & 2449171.04 & 586180.01 & 842234.6842 & 355557.8609 \\
\hline$A P=18$ & 17891.96 & 33987.36 & 2449328.06 & 586341,13 & 842232,7425 & 355559.4284 \\
\hline AP- 19 & 18113.19 & 34028.40 & 2449485.08 & 586502.26 & 842230.8009 & 355600.9959 \\
\hline AP.22 & 17490.56 & 33684.04 & 2449175.14 & $585 \$ 61.86$ & 842234.6985 & $3555 \$ 4.7143$ \\
\hline$A P=23$ & 17711.78 & 33725.09 & 2449332.16 & 586022.99 & 842232.7568 & 355556.2818 \\
\hline AP-24 & 17933.01 & 33766.13 & 2449489.18 & 586184.11 & 842230.8152 & 355557.8493 \\
\hline
\end{tabular}

LSU $=$ land survey unit.

\section{E.7. DOCUMENTATION}

Survey data will be documented in accordance with the procedures and reviews required by the DOE Contractor. A report will be prepared that describes the survey methods, results, and evaluation. The report will include the findings of the assessment, describe the materials surveyed and their condition, and justify the contamination-potential classification assigned. The data cvaluation will be included, along with the assessment of the quality assurance (QA)/quality control (QC) documentation. This report, or a summary of it, will also be included and referenced in the facility's baseline envirommental conditions documentation.

\section{E.8. QUALITY ASSURANCE}

All appropriate $\mathrm{QA} / \mathrm{QC}$ reviews to ensure the quality of the data gathered will be performed and documented. Survey instruments and methods specified in applieable RADCON operating and techrical procedures bave been decumented as to their ability to provide a $95 \%$ confidence level in detection of surface contamination at levels that meet the requirements of this protocol. Supporting data are provided on each survey form.

A DOE Contractor RADCON-Certiffed Health Physicist, or another designated bealth physicist, will review, evaluate, and validate the survey results, including assessment of the QAQC information and data, before generation of the radjological survey report. The final radiological surwey report will include the details of this assessnent. It will be provided to the DOE Contractor project QA manager, project manager, and site project health physicist for approval before its inclusion in the Environmental Baseline Survey report 


\section{APPENDIX F}

\section{RISK EVALUATION FOR PARCEL ED-4}




\section{CONTENTS}

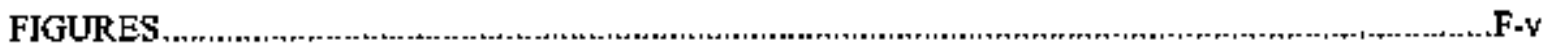

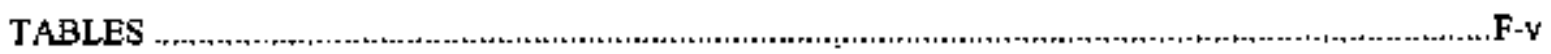

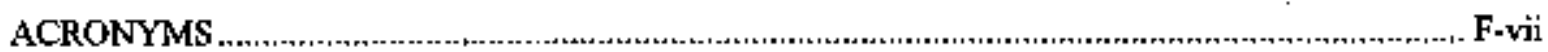

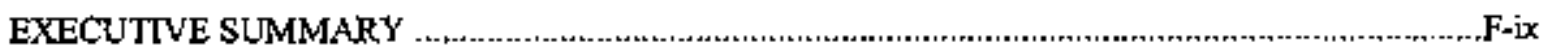

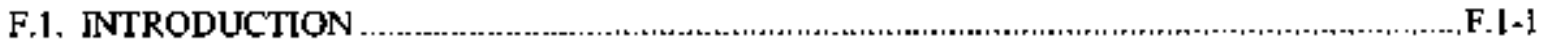

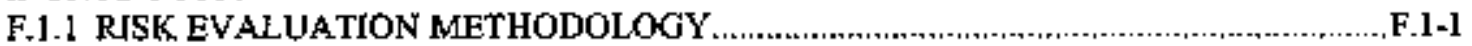

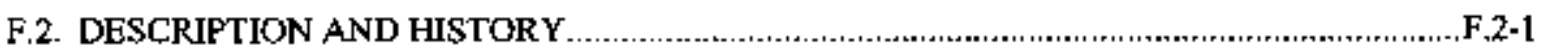

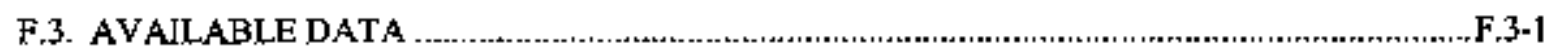

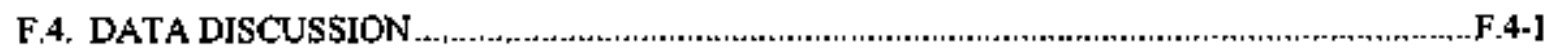

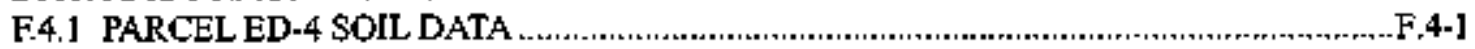

F.4.2 PARCEL ED-4 SEDIMENT DATA ............................................................................

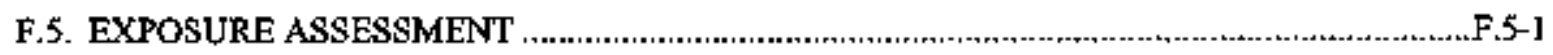

F.5.1 EXPOSURE SCENARIO EVALUATJON .....................................................5-1

F.5.1.1 Residential Scenario ...........................................................................5.

F.S.2 EXPOSURE PATHWAY JDENTIFJCATION .........................................................

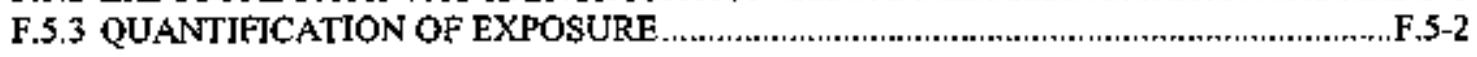

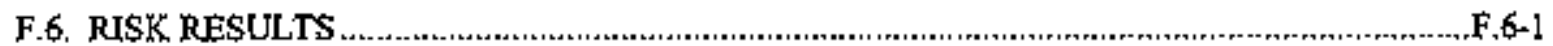

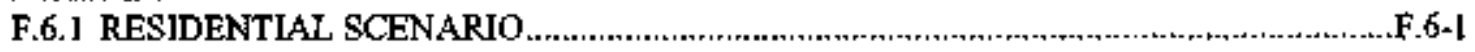

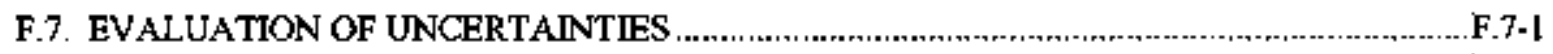

F.7.1 UNCERTAINTY IN THE SOURCE TERM …........................................................ $7-1$

F.7.2 UNCERTAINTY IN THE EXPOSURE ASSESSMENT …....................................... 7.1

F.7.3 UNCERTAINTY IN TOXICITY VALUES AND RISK PREDICTIONS ......................F.7-2

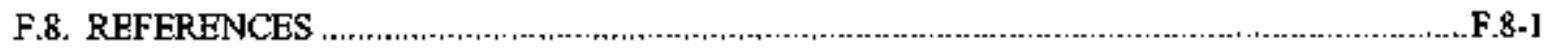




\section{FIGURES}

E.4.1 Parce] ED-4 soit and sediment sampling locations. ......................................................... F.4-2

\section{TABLES}

F.6.1 Results of residential risk sereen for ED-4 soils and sediment ..................................... F.6-3 


\title{
ACRONYMS
}

\author{
bgs below ground surface \\ CERCLA Comprehensive Environunental Response, Compensation, and Liability Act of 1980 \\ COPC contaminant of potential concern \\ CPD Clean Parcel Determintation \\ DOE U. S. Department of Entrgy \\ EBS Environmental Baseline Survey \\ EPA U.S. Environmental Protection Agency \\ EPC exposure point concentration \\ ETTP East Tennessee Technology Park \\ HI hazard index \\ HQ hazard quotient \\ PRG preliminary remedjation goal \\ RAGS Risk Assessment Guidance for Superfund \\ RL remediation level \\ ROD Record of Decision \\ TDEC Tennesset Departinent of Environment and Conservation
}




\section{EXECUTIVE SUMMARY}

The goal of this risk evaluation is to detemine the potential for adverse health effects associated with Parcel ED-4. The U. S. Department of Energy (DOE) is proposing to transfer title of this land parcel to the Heritage Center, LLC under a Clean Parcel Deternination (CPD). The intended land use for the property is industrial/conmercial. However, to support a CPD, a residential scentario was evaluated.

The methodology followed in perforning this risk evaluation included sereening the site data against nationally available preliminary remediation goals (PRGs), as well as remediation levels (RLs) developed for the East Tennessee Technology Park (ETTP) Zone I soils Record of Decision (ROD), to detenmine the need for a full risk calculation. The full risk calculation is conducted only when the constituents exceeding PRGs indicate the potential for elevated risks [cumulative excess lifetime cancer risk (ELCR) exceeding EPA's generally acceptable risk range or a bazard index (HI) above 1), or where no nationally recognized PRGs are available for the exposure scenario being considered.

The U. S. Environmental Protection Agency bas established a generally acceptable target risk ranige of E-04 to E-06 (also expressed as $10^{-4}$ to $10^{-6}$ ) and a generally acceptable $\mathrm{HI}$ of 1 . The ELCR is a value that represents the excess cancer incidence that might be expected due to the expossure scenario evaluated. $\mathrm{The} \mathrm{Hl}$ is a value that represents the potential for toxic effects to an exposed individual.

The analytical results for Parcel ED-4 soils and sediment indicated the cumulative risks were withit the acceptable risk range and the HI did not exceed 1 ; therefore, a full risk calculation was not necessary. As stated above, because the risk estimate did not exceed the generally acceptable risk range or exceed a HI of 1 , the risk evaluation was considered indicative of the low likelihood of adverse health effects associated with residential exposure to the Parcel ED-4 soils and sediments. The land parcel, therefore, is considered suitable for transfer as a Clean Parcel per the Comprehensive Environmental Response, Compensation, and Liability Act of 1980 (CERCLA) Sect. 120(h)(4). 


\section{F.1. INTRODUCTION}

The goal of this risk evaluation is to determine the potential for adverse health effects associated with Parcel ED-4, which is proposed for transfer by the U. S. Department of Energy (DOE). Specifically, the objectives of this evaluation are: (1) to determine exposure to constituents based on available data for the soils and sediment, and (2) to use these data to provide an estimate of the potential for adverse effects to human health. The risk calculations utilized in this evaluation are based on the document Risk Assessment Guidance for Superfiand (RAGS) [EPA 1989]. The following sections describe the process used to provide a quantitative analysis of the risks to human health from exposure to Parcel ED-4.

\section{F.I.I RISK EVALUATION METHODOLOGY}

The risk evaluation methodology utilizes a step-wise process in order to more efficiently determine if the property under consideration is suitable (from a health perspective) for transfer. As detailed below, the site data are screened against trigger levels fust to determine if further examination of the data is necessary. By virtue of the decision needed to be made when exannining the data (i.e., health protection), the screening process is conservative.

The risk evaluation method to support the title transfer of Parcel ED-4 includes analysis of both soil and sediment. A process agreed to by both DOE and the regulators [i.e., U. S. Environmental Protection Agency (EPA) and Tennessee Department of Euvironment and Conservation (TDEC)] is utilized in order to be consistenl with other programs (e.8., Environmental Management). Soil and sediment sampling results are compared with Preliminary Remediation Goals (PRGs) and/or site-specific remediation levels (RLs). PRGs are health-protective concentrations that have been developed by EPA Region 9 for the purpose of screening chemical concentrations to identify chemicals of potential concern. RLs are health-profective concentrations that have been establisbed in the site Recoris of Decision (RODs).

PRGs are developed based on a specific exposure scenario (ie. residential) and exposure pathways (soil ingestion, inhalation, and/or dermal contact) for a given level of risk and hazard [i.e., risk of 1E-06 and hazard quotient (HQ) of 0.1]. The risk represents the estimated probability of increased cancer incidences for the exposed population (i.e., risk of $1 \mathrm{E}-06$ means a 1-in-1,000,000 increased chance). The $\mathrm{HQ}$ is a measure of the potential for toxic effects from an individual contaminant, and the sum of $\mathrm{HQs}$ for multiple constituents is referred to as the hazard index (HI). An HI that exceeds I indicates the possibility that toxic effects may occur in the exposed propulation. The RLs use site-specific tata to develop bealth-protective concentrations for contaminants that are site-related and considered widespread. Because sife-specific data are used to develop RLs, they are higher in eoncentration than the PRGs.

As indicated above, the evaluation of risk and hazands is based on comparing soil and sediment sarnple results with PRGs or RLs. If there are constituents with concentrations in excess of the PRGs or RLs, further evaluation is conducted to ensure thal cumulative risks are within the acceptable risk range and the overall $\mathrm{HI}$ is below 1 for the facility. If the site data indicate the potential for clevated risks and/or hazards, a full risk calculation is conducted. The full risk calculation is based on an exposure assessment and identified exposure parameters (e.g., soil ingestion rate, exposure frequency, body weight, etc.) for the anticipated receptors. The results of the full risk calculation are then compared to the acceptable risk and hazard levels to determine the potential for adverse health effects associaled with soils and sediment in order to determine if the property is suitable for transfer. The following sections describe the process used to provide a quantitative analysis of the potential risks to human health while occtupying Parcel ED-4. 


\section{F.2. DESCRIPTION AND HISTORY}

A full description and history of Parcel ED-4, as well as site maps, are prestented in Chaps. 1 through 4 of the draft Environmental Baseline Survey (EBS) report for the ED-4 study area (DOE 2007, in progress). 


\section{F.3. AVAILABLE DATA}

The data available for the Parcel ED-4 study area consist of soil and sediment results from nine total sempling locations. Chapter 6 of the EBS provides a detailed evaluation of all available data, which are summarized in the following sections. 


\section{F.4. DATA DISCUSSION}

The available data for ED-4 are discussed in detail in Chap. 6 of the EBS and are summarized below. Duplicate analyses were conducted at some locations for quality assurance purposes. For the risk assessment, duplicate analyses were reduced to a single result (for each location, sample depth, and sampling date) in order to avoid biasing the dataset toward locations with duplicate analyses available. Where the original sample and duplicate were both detections, the larger detection was selected as the representative result. Where both the original and duplicate were non-detections, the sample with the lower detection limit was selected as the representative result. Additionally, the risk evaluation considered soil and stdiment data as a single media in the analysis of potential residential exposures.

\section{F.4.1 PARCEL ED-4 SOIL DATA}

Field sampling was conducted in Jime 2007 and generated soil analytical results from four locations (Fig. 4.1). Samples were collected from 0 to $0.5 \mathrm{ft}$ below ground surface (bgs) and analyzed for metals, organics, and radionuclides.

\section{F.4.2 PARCEL ED-4 SEDIMENT DATA}

The June 2007 sampling event also generated sediment analytical results from five locations and analyzed for metals, organics, and radionuclides (Fig. 4.1). 


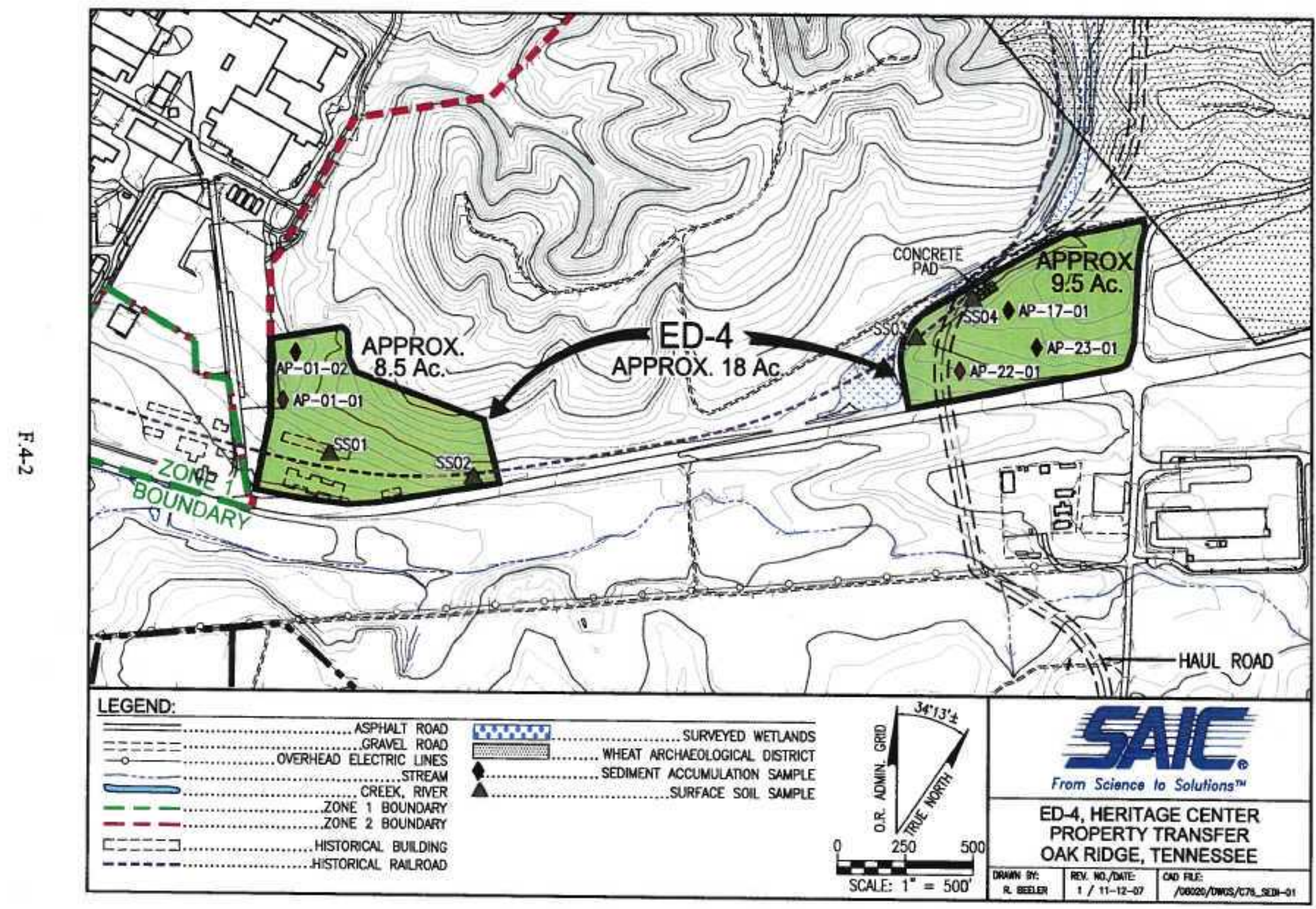

Fig. F.4.1. Parcel ED-4 soil and sediment sampling locations. 


\section{F.5. EXPOSURE ASSESSMENT}

An exposure assessment combines information about site characteristics and site-related data with exposure assumptions in order to quantify the intake of contaminants by a hypothetically exposed individual. The estimated exposure is based on the following:

- characterizing the exposure scenario based on site surveys,

- identifying complete exposure pathways based on assumed receptor activities and site-specific information, and

- quantifying receptor exposure based on exposure assumptions and chemical-specific data.

The steps in the exposure assessment are discussed in detail in the following sections.

\section{F.5.1 EXPOSURE SCENARIO EVALUATION}

Exposure scenarios are selected based on site surweys and anticipated uses of Parcel ED-4. ED-4 is being transferred for industrial uses. However, a residential scenario was considered for this land parcel to support a Clean Parcel Deteruination (CPD).

Exposures to the resident, while spending time outside and within the transfer area, were evaluated using soil and sediment sanpling results. Uncertainties associated with the exposure scenario evaluation arc presented in Chap. F.7.

\section{F.5.1.1 Residential Scenario}

The hypothetical residential scenario assumes that a resident may be present on Parcel ED-4 in the future. ${ }^{1}$ It is assumed that the resident is exposed to soils from 0 to $2 \mathrm{ft}$ bgs as well as sefiment, while hypothetically living in the area. Details associated with this theoretical residential scenario are presented in the following section

\section{F.5.2 EXPOSURE PATHWAY IDENTIFICATION}

Evaluating the exposure pathways requires describing the mecbanisa by which an individual may become exposed to contaminants associated with Pancel ED-4 transfer area soils and sediment. A complete exposure pathway requires the following:

- a source of contamination,

- a patbway of migration from the source of contamination to the exposure point,

- a receptor present at the exposure point, and

- an exposure mechanism at the exposure point.

If any one component of a complete exposure pathway is missing, then the pathway is considered incomplete. Only complete exposure pathways were evaluated in the risk scretn.

\footnotetext{
' It should be noled that the Quitclaim deed prohibits ressidential use.
} 
Complete exposure pathways associated with Parcel ED-4 soils and sediment include ingestion, inhalation, demal contact, and extemal exposure to ionizing radiation. The ingestion pathway is complete because contaminated media may be present, a receptor nuy be present on the parcei, and a receptor may contact and ingest contaminants from the media. The inbalation pathway is complete because contaminated media may be present, contaminants may become airbome, a receptor may be present on the parcel, and an individual may inhale contaminants that are in the air. The dermal pathway is complete because contaminated media may be present, a receptor may be present on the parcel, and a receptor may contact and dermally absorb contaminants from the media. External exposure to ionizing radiation is a complete exposure pathway because radionuclides may be present in media, ionizing radiation may be ernitted, and a receptor may be present to absorb the radiation. The following section describes how each of these exposure pathways was quantified in the risk screen.

\section{F.5.3 QUANTIFICATION OF EXPOSURE}

The evaluation of the ED-4 residential scenario included a comparison of detected concentrations with EPA Region 9 residential PRGs adjusted to a risk level of $10 \mathrm{E}-6$ and an HQ of 0.1 .

The residential exposure scenario assumes the following:

- the resident is present on Parcel ED-4 for 30 years as an adult, and for 6 years as a child;

- the resident is on-site for 350 d/year,

- the resident is outdoors exposed to solis for $1.5 \mathrm{~h} / \mathrm{d}$ as an adult, and $2.25 \mathrm{~h} / \mathrm{d}$ as a child;

- the resident ingests $100 \mathrm{mg} / \mathrm{d}$ and $200 \mathrm{mg} / \mathrm{d}$ of contaminated soil as an adult and child, respectively, and

- the resident inhales $20 \mathrm{~m}^{3} / \mathrm{d}$ as an adult and $15 \mathrm{~m}^{3} / \mathrm{d}$ as a child.

As described in the risk evaluation methodology in Sect. F.1.1, for the Parcel ED-4 study area soils and sediment, detecled concentrations were compared with EPA Region 9 residential PRGs adjusted to a risk level of $1 \mathrm{E}-06$ and an $\mathrm{HQ}$ of $0 . \mathrm{l}$, as well as site-specific RLs. Because the comparison indicated a low potential for adverse health effects, a full risk calculation was not required, as discussed in Chap. F.6. 


\section{F.6. RISK RESULTS}

The EPA has established a generally acceptable target risk range of E-04 to E-06 (also expressed as $10^{-4}$ to $10^{-6}$ ) and a target $\mathrm{HI}$ of $\mathrm{l}$. The following sections present the evaluation of site analytical data collected from the Parcel ED-4 study area

\section{F.6.1 RESIDENTLAL SCENARIO}

Hypothetical residential exposures associated with Parcel ED-4 soils and sediment may occur via ingestion, inbalation, dermal conlact, and external exposure. As discussed in the previous section, EPA Region 9 resideotial PRGs and ETTP RLs were used to screen the soils and sediment as follows:

- each detected result was compared with EPA Region 9 PRGs for the residential scenario adjusted to a risk level of $1 \mathrm{E}-06$ and an $\mathrm{HQ}$ of 0.1 ;

- detected results were compared with the RLs developed for the ETTP Zone I ROD: and

- detected results were compared with background levels.

As discussed in Sect. F.1.1, the dataset was evaluated to detemine if the generally acceptable risk range and $\mathrm{HI}$ of 1 were exceeded as follows:

- constituents with maximum detected concentrations above the RLs and/or PRGs wete evaluated to deternine the potential for a cumulative risk exceeding the acceptable risk range or an HI exceeding 1.

- constituents with a maximum detected concentration below background were elirninated.

Table F.6.1 presents the results of the Parcel ED-4 risk evaluation based on residential cxposures and indicates the following:

- No constituents wete determined to exceed the RLs.

- Constituents exceeding the PRG and/or background values were selenium, thallium, benzo(a)pyrene, radium-226, technetium-99, and uranium-238.

- Selenium analytical results included laboratory qualifiers indicating thal matrix interference and sample dilution hindered accurate quantification of media concentrations. Process knowledge at ED-4 does nol indicate a likely source of selenium to account for these elevated levels above background criteria; however, selenium is included in the evaluation for completeness.

- Radium-226 is a site-wide contaminant based on backgound considerations (DOE 2002a) and is not considered sitc-specifie to ED-4.

- Based on consultation with EPA and verification utilizing the RAIS risk estimator (tttp//rais.orsl.gov/cgi-bin/prg/for_cnt_data), site concentations with maximum concentrations that excecded the residential PRG wert cvaluated to determine the potential for adverse health effects associated with exposure to maximum delected concentrations. The results indicate a curnulative 
ELCR within the acceptable risk range and $H I<I$ associated with constituents detected in ED-4 soils and sedimeat.

Because the potential residential risks associated with ED-4 soil and sediment did not exceed the generally acceptable risk range of $\mathrm{E}-04$ to $\mathrm{E}-06$ or $\mathrm{HI}$ of $\mathrm{l}$, no further evaluation was needed, and a full risk calculation was not conducted. The screening was considered indicative of the low likelihood of adverse health effects assocjated with residential exposure to Pancel ED-4 soils and sediment. Parcel ED-4 is, therefore, considered suitable for tuansfer as a Clean Parcel per Comprehensive Environtrental Response, Compensation, and Liability Act of 1980 (CERCLA) Sect. 120(h)(4). 
Table F.6.1. Results of residential risk screen for ED-4 soiks and sedlalent

\begin{tabular}{|c|c|c|c|c|c|c|c|c|c|c|c|c|}
\hline Constiment & $\begin{array}{c}\text { Frequency } \\
\text { of detect }\end{array}$ & $\begin{array}{c}\text { Milniminum } \\
\text { detect }\end{array}$ & $\begin{array}{c}\text { Maximum } \\
\text { deloxi }\end{array}$ & $\begin{array}{c}\text { Avernge } \\
\text { detected } \\
\text { resilt. }\end{array}$ & $\begin{array}{l}\text { Background } \\
\text { concentration }\end{array}$ & $\begin{array}{l}\text { Frequeacy } \\
\text { of deteds } \\
\text { excetibes } \\
\text { backs round }\end{array}$ & $\begin{array}{c}\text { Maximum } \\
\text { RL }\end{array}$ & $\begin{array}{c}\text { Freguency } \\
\text { of detects } \\
\text { exceeding } \\
\text { maxumur } \\
\text { RL } \\
\end{array}$ & $\begin{array}{c}\text { Averag* } \\
\mathbf{R L}\end{array}$ & $\begin{array}{l}\text { Freqoency } \\
\text { of detecls } \\
\text { exceeding } \\
\text { everate RL }\end{array}$ & $\begin{array}{c}\text { Residential } \\
\text { PRG } \\
\text { (Rlsk }=1 E-06 \\
\text { HQ }=0.4 \text { ) }\end{array}$ & $\begin{array}{l}\text { Frequency } \\
\text { of detects } \\
\text { exceeding } \\
\text { PRG linit }\end{array}$ \\
\hline Selenium & $9 / 9$ & $39.5 \mathrm{~J}$ & $126 \mathrm{~J}$ & 86.46 & 1.47 & $9 j 9$ & NA & $N_{A}$ & NA & $\mathrm{NA}$ & 39.1 & 99 \\
\hline Thalliurn & $1 / 9$ & $1.46 \mathrm{~J}$ & $1.46 \mathrm{~J}$ & 1.46 & $0.4 \mathrm{U}$ & $1 / 9$ & NA & NA & $\mathrm{NA}$ & NA & 0.516 & 149 \\
\hline Benzo(a)pyizese & $6+8$ & 0.143 & $0.269 \mathrm{~J}$ & 0.18 & NA & NA & $\mathrm{N} A \mathrm{~A}$ & NA & NA & $N A$ & 0.0621 & $6 / 8$ \\
\hline Radium-226 & 99 & 0.688 & 1.47 & 0.97 & 1.25 & $1 / 9$ & 15 & org & 5 & 099 & 0.0124 & 9F: \\
\hline Techntetiunst-99 & $5 / 9$ & 0.0388 & 0.387 & 0.12 & NA & $\mathrm{NA}$ & NA & NA & NA & NA & 0.25 & $1 / 9$ \\
\hline Uranism-238 & $9 / 9$ & I.4 & 3.08 & 2.13 & 1.47 & 899 & 500 & ON & 50 & arg & 0.743 & $9 / 9$ \\
\hline
\end{tabular}

NA m not applicable or not available.

at - Cobstituent is considered a site-wide contaminent based on beckground considerations and is nol site specifte to ED-4 (DOE 2002a).

PRG - preliminary remediation goal. Yalues presented here are from U. S. Environmental Protection Agerncy Region 9 for residential exposure adjusted for a tisk level of IE.06. and hazerd quotient of 0.1 .

RL क rentedial lewel from ETTP Zonel ROD (DOE 2002n). 


\section{F.7. EVALUATION OF UNCERTAINTIES}

The estimation of uncertainty, whether quantitative or qualitative, is fundamental to scientific activities that involve measured or assessed quantities. Estimates of risk are conditional based on a number of assumptions conceming exposure. Generation of a point estimate of risk, as has been done in this screening-level assessment, bas the potential to yield under- or overestimates of the actual value and can lead to improper decisions. Therefore, it is nexessary to specify the assumptions and uncertainties inherent in the screening-level evaluation process to place the riste estimates in perspective and ensure that anyone making risk-management decisions is well informed.

Uncertainty about environumental risk estimates is known to be at least an order of magnitude or greater (EPA 1989). The evaluation of uncertainties for the assessment is qualitative, since the resource requirements necessary to provide a quantitabive statistical uncertainty analysis for this sndy area would generally outweigh the benefits. The focus of the discussion in this section will be on the important variables and assumptions that contribute most to the overall uncertainty.

\section{F.7.1 UNCERTAINTY IN THE SOURCE TERM}

Several uncertainties are associated with the data set and the data evaluation process. These uncertainties include the selection of $\mathrm{COPCs}$ and the determination of the EPC.

Although the data evaluation process used to select COPCs adberes to established procedures and gujdance, it also requires making decisions and developing assumptions on the basis of historical information, process knowledge, and best professional judgment about the data. Uncertainties are asssociated with all such assumptions. The backeround concentrations and PRGs used to screen analytes are also subject to uncertainty. The toxicity values used in the derivation of PRGs are subjeet to change; as additional information (from scientific research) becomes available, these periodic changes in toxicity values may cause the PRG values to change as well, causing increased uncertainty in the data screening process.

Representative concentrations and other statistics are calculated in this risk screen based on the assuniption that the samples collected are truly random samples. Some of the data may not have been taken randomly, but rather may have come from biased sampling, aimed at identifying high contaninant oncentration locations.

This evaluation has been performed using only the COPCs with avajlable toxicity data Radionuclides that are short-lived isotopes were eliminated from the datiset, along with daughter products of isotopes that include the contribution of the daughter in the PRG calculation to overestimating their contribution to the overall risks.

\section{F.7.7 UNCERTAINTY IN THE EXPOSURE ASSESSMENT}

For each exposure pathway, assumptions are made concerning the parameters, the routes of exposure, the amount of contaninated media an individual can be exposed to, and intake rates for different routes of exposture. In the absence of site-specific data, the assumptions used in this assessment are consistent with EPA-appoved pararieters and default values. Whes several of these upper-bound values are combined in estimating exposure for any one pathway, the resulting risks can be in excess of the 99th percentile and, therefore, outside the range that may be reasonably expected. 
The guidance values for intake rates and exposure parameters are assumed to be representative of the hypothetical populations evaluated. All contaminant exposures and intakes are assumed to be from the site-related exposure media (i.e., no other sources contribute to the receptor's risk). Even if these assumptions are true, other areas of uncertainty may apply. Selected intake rates and population characteristics (i.e., weight, life spar, and activities) are assumed to be representative of the exposed population. The consistent conservatism used in the estimation of these parameters generally leads to overestimation of the potential risk to the postulated receptors.

\section{F.7.3 UNCERTAINTY IN TOXICITY VAIUES AND RISK PREDICTIONS}

Uncertainty in the vahues used to represent the dose-response relationship will bighly inapact the risk estimates. These uncertainties are contaminant-specific and are embedied in the toxicity value. The factors that are incorporated to represent sources of uncertainty include the source of the data, duration of the study, extrapolations from short- to long-term exposures, intrahuman or interspecies variability, and? other special considerations. In addition, toxicity varies with the chemical form.

Uncertainties related to the sumnation of carcinogenic risk and non-carcinogenic hazard estimates across contaminants and pathways are a primary uncertainty in the risk characterization process. In the absence of infomation on the toxicity of specific chemical mixtures, additive (curnolative) risks are assumed (EPA 1989).

Limitations of the additive risk approach for exposure to multiple chemicals include the following:

1. The slope factors may represent the mean but often represent the upper 95 th percentile estimate of potency (the central estimate on the mean for radionuclides), so the summation can result in an excessively conservative estimate of lifetime risk.

2. The reference doses do not have equal accuracy or precision and are not based on the same severity of effects.

3. The effects of a mixture of carcinogens are unknown, and possible interactions could be synergistic or antagonistic.

Despite these limitations and the general unavailability of data on these interaclions, summations were performed for the earcinogenic risks and chemical bazards presented in the risk screen. This approach is consistent with RAGS (EPA 1989).

In order to avoid double-counting the short-ljved daughters of specific isotopes, the daughters were excluded from the COPC list if analytical results for the parent were available; only daughters as defined by EPA (2001) were excluded. As a special casse, the ${ }^{232}$ Th decay chain was evaluated as " $232 \mathrm{~Tb}+\mathrm{D}$ " (which combines the slope factors for ${ }^{392} \mathrm{Th},{ }^{223} \mathrm{Ra}+\mathrm{D}$, and ${ }^{278} \mathrm{Th}+\mathrm{D}$ ) when calculating risks. When evaluating data for "2232 $\mathrm{Th}+\mathrm{D}$," a conservative approach was used, whereby the largest concentration among ${ }^{232} \mathrm{Th}$, ${ }^{228} \mathrm{Ra}$, and ${ }^{225} \mathrm{~Tb}$ was used to determine the maximum detected conctatration and to estimate all summary statistics. Another special consideration for radioisotopes was to eliminate ${ }^{80} \mathrm{~K}$ from the COPC list, as it was considered to be naturally occurring and, therefore, was not considered to be a COPC. 


\section{F.8. REFERENCES}

DOE (U. S. Department of Energy) 1999. Remedial Investgation Report for the East Tennessee Technology Park, Oak Ridge. Tennessee, DOE/OR/01-1778/VI\&D2, U. S. Department of Energy, Office of Environmental Management, Oak Ridge, TN, January.

DOE 2002a Record of Decision for Interim Actions in Zone 1, East Tennessee Technology Park, Oak Ridge, Tennessee, DOE/OR/OI-1997\&D2, U. S. Department of Energy, Office of Environmental Management, Oak Ridge, TN.

DOE 2002b. Oak Ridge Performance Management Plan, Rev. 6, U. S. Department of Entrgy, Office of Environmental Management, Oak Ridge. TN, August 28.

DOE 2007 (in progress). Draft Environmental Baseline Survey Report for the Title Transfer of Land Parcel ED-4 at the East Tennessee Technology Park. Oak Ridge, Tennessee, DOE/OR/01-2304, U. S. Department of Energy, Office of Nuclear Fuel Supply, Oak Ridge, TN.

EPA (U. S. Environrnental Protection Agency) 1989. Risk Assessment Guidance for Superfund, Vol. I: Human Health Evaluation Manual (Part A), EPA/540/1-89/002, Washington, D.C.

EPA 1991. Risk Assessment Guldance for Superfind, Volume 1: Human Heallh Evaltation Manwal, Stpplemental Guidance, Standard Default Exposure Factors, Interim Final, OSWER Directive 9285.6-03, Office of Emergency and Remedial Response, Washington, D.C.

EPA 1992. Supplemental Gutdance to RAGS: Calculating the Concentration Term, OSWER Directive 9285.7-081, Office of Solid Waste and Emergency Response, Washington, D.C.

EPA 1996. Soll Screening Guidance: Technical Bachground Document, Office of Solid Waste and Emergency Response, Washington D.C.

EPA 1997. Exposure Factors Handbook, EPA/600/P-95/002Fa, Office of Research and Development, National Center for Environmental Assessment, Washington, D.C. (found on the World Wide Web at http://www,epa.gov/ncea/pdfs/efh/front. pdf).

EPA 2000. Supplemental Guidance to RAGS: Region IV Bulletins, Human Health Rish Assessment, Atlanta, GA (available on the World Wide Web at htp://www.epa.goviregion/wasle/ots/healibul htm).

EPA 2001. Heakth Effects Assessment \$ummary Tables (HEAST), avaikable on the World Wide Web at http://www.qpa.gov/radiation/heast/index.httol.

EPA 2002a. Guidance for Comparing Background and Chemical Concentrations in Soil for CERCLA Sites, EPA 540-R-01-003, Office of Solid Waste and Emergency R sonse, Washinglon, D.C., found on the Worid Wide Web at http//twww.epa.gov/superfund/programs/risk/background.pdf.

EPA 2004a, Reglon 9 Preliminary Remediation Gaals (PRGs), Oct. 2004 update, created by Stanford J. Sinucker, found on the World Wide Web al hitt;/www.epa.gow/Region9/waste/sfund/prg/index.htm. 
EPA 2004b. Risk Assessment Guidance for Superfund Volume I: Human Health Evaluation Manual (Part E, Supplemental Gutdance for Dermal Risk Assessment) Final, OSWER 9285.7-02EP, Office of Superfund Remediation and Technology Innovation, Washington, D.C., August, found on the Worid Wide Web at http:/wow .epagov/superfund/programs/risk/ragse/introduction.pdf. 
DOE/OR/01-2304

FINAL-CONCURRED

\section{RECORD COPY DISTRIBUTION}

File-EMEF DMC-RC 